\title{
Nanostructured Inorganic Metal Halide Perovskites for Optoelectronic Applications
}

by

Parth Vashishtha

\author{
A thesis \\ submitted to the Victoria University of Wellington \\ in fulfilment of the \\ requirements for the degree of \\ Doctor of Philosophy \\ in Chemistry
}

Victoria University of Wellington

2018 

For my sister Kapila Vashishtha 



\begin{abstract}
Semiconductor quantum dots have proven to be promising materials for optoelectronic devices, such as light emitting devices (LEDs) and solar cells, due to their thin linewidth of emission, high photoluminescence quantum yield and high absorption coefficient. Over the last decade, perovskite crystals have gained significant attention due to their extraordinary optoelectronic properties. Therefore, perovskite nanocrystals combine the advantage of both crystalline perovskite and quantum dots. Here, we synthesised high quantum yield (50 - 80 \%) monodispersed $\mathrm{CsPbX}_{3}(\mathrm{X}=\mathrm{Cl}, \mathrm{Br}, \mathrm{I})$ quantum dots, with tuneable emission spectra over the entire visible region, by a colloidal synthesis method. We have then successfully processed them to produce thin films as the emitting layer in an organic LED-type device architecture. Most importantly, we demonstrated field induced halide separation in mixed halide $\mathrm{CsPb}(\mathrm{Br} / \mathrm{I})_{3} \mathrm{NCs}$ which is the reason color instability in these LEDs.

Perovskite nanocrystal LEDs were found to have low external quantum efficiency (EQE) due to their bulky ligands. As a result, Ruddlesden-Popper (RP) phase layered perovskite was investigated to increase the EQE over perovskite QD LEDs. As a result, we constructed RP perovskite phase $\mathrm{CsPbX}_{3}$ LEDs with emission through the entire visible spectrum (460-700 nm). Colour tuning was achieved by taking advantage of both quantum confinement effect and halide mixing. The EQE of these LEDs outperformed the literature values in the blue and blue-green spectral regions, with relatively long life time.

We also invented a novel perovskite nanocrystals made from thallium lead halide by replacing caesium with thallium. These materials are potential candidates for various optoelectronic applications. Size-, shape-, and composition- tun-
\end{abstract}


ing in these nanocrystals were performed by varying the reaction conditions and mixing the halide composition. A weak confinement was observed in these NCs. Additionally, we have shown the application of $\mathrm{TlPbI}_{3}$ nanowires as photoconductors.

Collectively, this thesis includes the synthesis of various types of inorganic metal halide perovskite nanostructures followed by their implementation into working optoelectronic devices, specifically LEDs. 


\section{Publications}

The following articles have been published from this thesis:

\section{Chapter 2:}

Butkus J, Vashishtha P, Chen, K, Gallaher JK, Prasad SK, Metin DZ, Laufersky G, Gaston N, Halpert JE, Hodgkiss, JM. The Evolution of Quantum Confinement in $\mathrm{CsPbBr}_{3}$ Perovskite Nanocrystals. Chemistry of Materials 2017.

Contribution: Synthesis of size dependent nanocrystals with XRD, TEM, static state absorption and photoluminescence spectroscopy, and writting of related parts of the publication.

Vashishtha P \& Halpert, JE. Field-Driven Ion Migration and Color Instability in Red-Emitting Mixed Halide Perouskite Nanocrystal Light-Emitting Diodes. Chemistry of Materials 2017. Contribution: Synthesis of size dependent nanocrystals with XRD, TEM and, static state absorption and photoluminescence spectroscopy.

Contribution: Synthesis of nanocrystals, fabrication of LEDs, data analysis, and writing the manuscript.

\section{Chapter 3:}

Vashishtha P \& Halpert, JE. Field-Driven Ion Migration and Color Instability in Red-Emitting Mixed Halide Perouskite Nanocrystal Light-Emitting Diodes. Chemistry of Materials 2017.

Contribution: Synthesis of nanocrystals, fabrication of LEDs, data analysis, and writing the manuscript. 


\section{Chapter 4:}

Vashishtha P \& Halpert JR, High Efficiency Blue and Green LEDs Using RuddlesdenPopper Inorganic Mixed Halide Perovskites with Butylammonium Interlayers, under review process 2018.

Contribution: Synthesis of perovskite material, fabrication of LEDs, data analysis, and writing the manuscript.

\section{Chapter 5:}

Vashishtha P, Metin DZ, Cryer ME, Chen, K, Hodgkiss J, Gaston N, Halpert JE. Shape-, Size-, and Composition-Controlled Thallium Lead Halide Perovskite Nanowires and Nanocrystals with Tunable Band Gaps. Chemistry of Materials 2018.

Contribution: Synthesis of nanocrystals and nanowires, microscopy and spectroscopy measurements with crystallography analysis, and writing the related parts of the publication.

\section{Appendix-1:}

Ma Y, Vashishtha P, Shivarudraiah SB, Chen K, Liu Y, Hodgkiss JM, Halpert JE. A Hybrid Perovskite Solar Cell Modified With Copper Indium Sulfide Nanocrystals to Enhance Hole Transport and Moisture Stability. Solar RRL 2017.

Contribution: Synthesis of $\mathrm{CuInS}_{2}$ nanocrystals, XRD, TEM, UV-Vis measurements on NCs, and writing the related parts of the publication. In addition, contributed in the back over of the journal. 


\section{Acknowledgments}

This work would have not been possible without the support of Victoria University of Wellington and the MacDiarmid Institute for Advanced Materials and Nanotechnology, New Zealand.

Firstly, I would like to thank my advisor Prof Jonathan E. Halpert for the continuous support during my research. It has been an honour to be his first PhD student. He has always been helping me time to time while providing me enough freedom to pursue my research goals. He was always very enthusiastic towards my achievements. Whenever I needed, he was always available to meet. Only because of his motivation and active involvement, I was able to publish several journal publications. In addition, he also helped me to file the patents on my inventions.

I am thankful to my Victoria supervisor Prof Thomas Nann, who has always been helpful to me during my PhD. Whenever I needed him, he was always there for me to discuss. His research group was always very helpful to me in carrying out the PL quantum yield measurement.

I would also like to thank our entire Halpert research group from 2015 to 2018. My special thanks goes to Matthew Cryer and Dr Yingzhuang Ma for interesting discussions on research ideas. I would also like to thank Dr Kai Chen for always giving me great ideas in the field of perovskite optoelectronics. In addition, Dr Chen has also helped me in conducting ultrafast photoluminescence spectroscopy for several nanocrystal samples. 
viii

My sincere thanks also goes to my secondary supervisor Prof Justin Hodgkiss, who provided me with valuable discussion and always made sure that my samples were getting characterised using ultrafast spectroscopy from his group. A big thank goes to Dr Justinas Butkus for transient absorbance measurement on my perovskite nanocrystals sample. I also like to thanks Dani Z. Metin for computational analysis on my perovskite nanocrystals.

I am very thankful to David Flynn for his valuable time and patience with me during the electron microscopy training. A big thank goes to Dr Anna Henning for the valuable discussion on crystallographic analysis on my nanocrystals. This work would have not been possible without the contribution from Dr Bruce Charlier, School of Geography, Environment and Earth Science, for ICP-MS measurements on my nanocrystals. A special thank to Vaneeza Asif for helping me polish up the figures and motivating me during the $\mathrm{PhD}$.

I want to thank my friends from New Zealand and overseas, specially, Vaibhav Bhugra, Shalini Divya, Rohan Borah, Mima Kurian, Dr Akash Bachuka, Apoorva Sharma, Dr Yatin J. Mange, Silvina Pugliese, Valentin Tannieres, Dr Yuta Nakayasu, Sunil Shivarudraiah, and Rishabh Bhatia to make my $\mathrm{PhD}$ life less stressful time to time from 2015 to 2018. I am grateful to Ashish Pateriya and Shruti Pateriya to make me feel like Home in New Zealand during my PhD.

Finally, I must thank my parents for their support during my entire career. Their love and encouragement was always one of the biggest motivations for my $\mathrm{PhD}$. I also want to thank my sister Kapila Vashishtha and brother Hrsh Vashishtha, who have always encouraged me during my $\mathrm{PhD}$. 


\section{Contents}

1 Background and Methods 1

1.1 Introduction . . . . . . . . . . . . . . . . 1

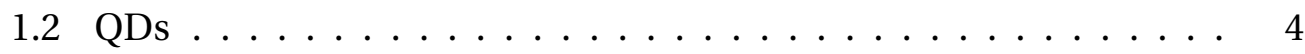

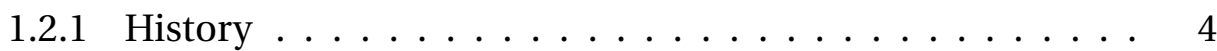

1.2.2 Shape Control of NCs $\ldots \ldots \ldots \ldots \ldots$

1.2 .3 Density of States . . . . . . . . . . . . . . 8

1.2.4 Band Gap Tuning . . . . . . . . . . . . . . . . . . . . 15

1.2.5 Colloidal Synthesis Method for NCs . . . . . . . . . . 18

1.3 Metal Halide Perovskite . . . . . . . . . . . . . . . . . . . 20

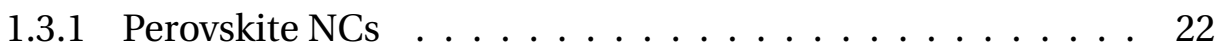

1.4 LEDs . . . . . . . . . . . . . . . . . . . . . . 23

1.4.1 Working Principle . . . . . . . . . . . . . . 25

1.4 .2 History of LEDs . . . . . . . . . . . . . . . . . 26

1.4 .3 Perovskite in LEDs $\ldots \ldots \ldots \ldots \ldots$

1.4.4 Carrier Recombination in Semiconductors . . . . . . . . . . 32

1.5 Solar Cells . . . . . . . . . . . . . . . . . . . . . . . . 34

1.5.1 Carrier Generation in Solar Cells . . . . . . . . . . . . . 34

1.5.2 J-V Characteristic of a Solar Cell . . . . . . . . . . . . 35

1.5.3 Perovskites in Solar Cells . . . . . . . . . . . . . . . . . 36

1.6 Methods . . . . . . . . . . . . . . . . . . 38

1.6 .1 TEM . . . . . . . . . . . . . . 38

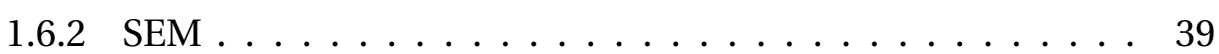

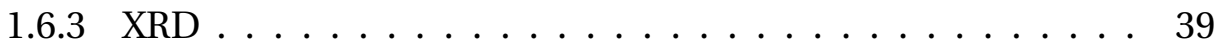


1.6.4 UV-Visible Spectroscopy . . . . . . . . . . . . . . . 41

1.6 .5 PL Spectroscopy . . . . . . . . . . . . . . . . . . 42

1.6.6 J-V Characteristic . . . . . . . . . . . . . . . . 43

1.6 .7 EL Measurement . . . . . . . . . . . . . . . . . 44

2 Size and Composition Dependent $\mathrm{CsPbX}_{3}(\mathrm{X}=\mathrm{Cl}, \mathrm{Br}, \mathrm{I})$ Nanocrystals 49

2.1 Introduction . . . . . . . . . . . . . . . . . . . . 49

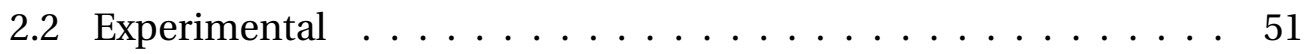

2.2.1 Synthesis of Size Dependent $\mathrm{CsPbBr}_{3}$ NCs and Microcrystalline Film . . . . . . . . . . . . . . . . . . . 51

2.2.2 Synthesis of Composition Dependent Perovskite NCs . . 54

2.2.3 Synthesis of $\mathrm{CsPbX}_{3} \mathrm{NWs} \ldots \ldots \ldots \ldots \ldots$

2.3 Results and Discussion . . . . . . . . . . . . . . . . . 55

2.3.1 Morphological Characterisation on Size Controlled NCs . . 55

2.3.2 Optical Spectroscopy on Size Controlled $\mathrm{CsPbBr}_{3} \mathrm{NCs}$. . . 60

2.3.3 Morphological Characterisation on Halide Composition Dependent Perovskite NCs . . . . . . . . . . . . 65

2.3.4 Optical Characterisation on Halide Composition Dependent Perovskite NCs . . . . . . . . . . . . . . . . . 69

2.3.5 Characterisation on Perovskite NWs . . . . . . . . . . 71

2.4 Conclusion . . . . . . . . . . . . . . . . . . 74

3 Colour Instability in $\mathrm{CsPbX}_{3}$ Perovskite Nanocrystal LEDs 75

3.1 Introduction . . . . . . . . . . . . . . . . . 75

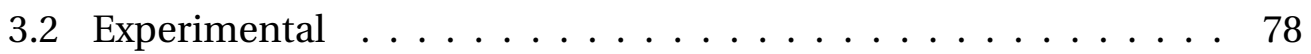

3.2.1 Fabrication of LEDs $\ldots \ldots \ldots \ldots \ldots$

3.3 Results and Discussion . . . . . . . . . . . . . . . . . . 81

3.3.1 Device Performance . . . . . . . . . . . . . . . . . 82

3.3.2 EL Peak Shifting in Mixed Halide Red LEDs . . . . . . . . . 86

3.3.3 Temperature Dependent PL Study on Mixed Halide NCs . . 90

3.3.4 Halide Composition and Voltage dependent EL Studies . . 92

3.3.5 Colour Instability in High Bromide Content LED (O558) . 98 
3.3.6 Stability Test on Mixed Halide LEDs . . . . . . . . . . . . 102

3.4 Conclusion . . . . . . . . . . . . . . . . . . . . . . . 103

4 Quasi-2D Inorganic Metal Halide Perovskite LEDs 105

4.1 Introduction . . . . . . . . . . . . . . . . . . 105

4.2 Experimental . . . . . . . . . . . . . . . . . 109

4.2 .1 Materials . . . . . . . . . . . . . . . . . 109

4.2 .2 Method . . . . . . . . . . . . . . . . . . 109

4.3 Results and Discussion . . . . . . . . . . . . . . . . . . . 113

4.3.1 Morphological Characterisation of RP Perovskite . . . . . 113

4.3.2 Optical Characterisation of RP Perovskite Thin Films . . . . 117

4.3.3 LED Performance . . . . . . . . . . . . . . . . . . . . 120

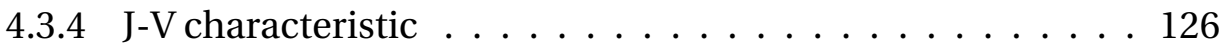

4.3 .5 Ion Migration $\ldots \ldots \ldots \ldots \ldots \ldots$

4.3 .6 Stability Test . . . . . . . . . . . . . . . . . . 129

4.4 Conclusion . . . . . . . . . . . . . . . . . . 130

5 Shape-, Size-, and Composition-Controlled Thallium Lead Halide Nanowires and Nanocrystals with Tunable Band Gaps $\quad 133$

5.1 Introduction . . . . . . . . . . . . . . . . . . . . . . . . 134

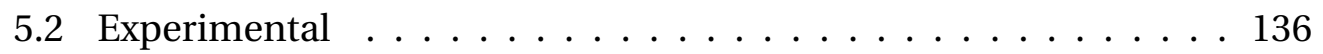

5.2.1 Synthesis of $\mathrm{Tl}_{3} \mathrm{PbI}_{5}$ Perovskite NWs . . . . . . . . . 136

5.2 .2 Method . . . . . . . . . . . . . . . . . . . . 137

5.2 .3 Synthesis of $\mathrm{TlPbI}_{3} \mathrm{NWs} \ldots \ldots \ldots \ldots$

5.2.4 Inductively Coupled Plasma Mass Spectrometry (ICP-MS) . 139

5.2 .5 Electrical Measurements . . . . . . . . . . . . . . 140

5.3 Results and Discussion . . . . . . . . . . . . . . . . . . . 141

5.3.1 Morphological Characterisation on Thallium Lead Iodide 142

5.3 .2 Optical Characterization . . . . . . . . . . . . . . . . . 155

5.4 Application . . . . . . . . . . . . . . . . . . . . . . . 162

5.5 Conclusion . . . . . . . . . . . . . . . . . 165 
6 Conclusion and Future Work 167

7 Appendix 173

7.1 Appendix 1- Improving the Performance of Perovskite Solar Cells using CIS NCs . . . . . . . . . . . . . . . . . 173

7.1 .1 Introduction . . . . . . . . . . . . . 173

7.1 .2 Experimental . . . . . . . . . . . . . . . 174

7.1.3 Results and Discussion . . . . . . . . . . . . . . 177

7.1.4 Conclusion . . . . . . . . . . . . . . . . 181

7.2 Appendix 2- $\mathrm{Er}^{3+}$ Doping in $\mathrm{Tl}_{3} \mathrm{PbBr}_{5} \mathrm{NCs} \ldots \ldots \ldots 181$

7.2.1 Synthesis of $\mathrm{Er}^{3+}$-doped $\mathrm{Tl}_{3} \mathrm{PbBr}_{5} \mathrm{NCs} \ldots \ldots \ldots \ldots 18 \ldots$

7.2 .2 Results and Discussion . . . . . . . . . . . . . . . 182

7.3 Conclusion . . . . . . . . . . . . . . . . . . . . . . . . 184 


\section{List of Figures}

1.1 An illustration of micelle and reverse micelle structure. . . . . . . 6

1.2 Density of states in a 3-dimensional semiconductors. . . . . . . 8

1.3 Density of states in 2 dimensional semiconductors. . . . . . . . . . 12

1.4 Density of states in 1 and 0 dimensional semiconductors. . . . . . 14

1.5 An illustration of band gap tuning in NCs . . . . . . . . . . . . 16

1.6 LaMer diagram for the growth of NCs. . . . . . . . . . . . . . 19

1.7 Crystal structure of the halide perovskite. . . . . . . . . . . 21

$1.8 \mathrm{CsPbX}_{3} \mathrm{NCs}$ under UV lamp. . . . . . . . . . . . . . . . . . 22

1.9 A photograph of most common light sources. . . . . . . . . . . . 24

1.10 A schematic for working principle of LEDs. . . . . . . . . . . . . . 25

1.11 Principle of YAG white light LEDs. . . . . . . . . . . . . . . . 28

1.12 A schematic of organic light emitting diode. . . . . . . . . . . . 29

1.13 A schematic of QD light emitting diode. . . . . . . . . . . . . . 29

1.14 An illustration for carrier recombination in semiconductors. . . . 33

1.15 Working principle of a solar cell. . . . . . . . . . . . . . . . 35

1.16 NREL chart for the efficiency of solar cell. . . . . . . . . . . . . . 37

1.17 Basic principle of XRD . . . . . . . . . . . . . . . . . . . 40

1.18 Basic principle of PL spectrophotometer. . . . . . . . . . . . . . 42

1.19 standard J-V curve of perovskite LEDs. . . . . . . . . . . . . . . 44

1.20 Graphs for the calculation of Perovskite LED metrics. . . . . . . . . 45

1.21 Human spectral sensitivity to colour and a CIE 1931 diagram . . . 48

2.1 A synthesis method of $\mathrm{CsPbBr}_{3}$ NCs. . . . . . . . . . . . . . . . . 52 
2.2 TEM images of $\mathrm{CsPbBr}_{3} \mathrm{NCs}$ washed by different solvents. . . . . 56

2.3 TEM images of fusion in $\mathrm{CsPbX}_{3}$ NCs. . . . . . . . . . . . . 57

2.4 TEM analysis on size controlled $\mathrm{CsPbBr}_{3}$ NCs. . . . . . . . . . . 58

2.5 XRD spectra of $\mathrm{CsPbBr}_{3} \ldots \ldots \ldots \ldots \ldots$

2.6 UV-Vis and PL spectra of size controlled $\mathrm{CsPbBr}_{3}$ NCs. . . . . . . . 61

2.7 Quantum confinement estimation of $\mathrm{CsPbBr}_{3}$ NCs. . . . . . . . . 63

2.8 Ultrafast transient absorption spectroscopy of $\mathrm{CsPbBr}_{3}$ NCs. . . . 64

2.9 TEM micrographs of mixed halide perovskite NCs. . . . . . . . . 66

$2.10 \mathrm{CsPbX}_{3} \mathrm{NCs}$ under UV lamp. . . . . . . . . . . . . . . . . 67

2.11 XRD spectra of $\mathrm{CsPbX}_{3}$ mixed halide NCs. . . . . . . . . . . . 68

2.12 Absorption spectra of $\mathrm{CsPbX}_{3}$ mixed halide NCs. . . . . . . . . 70

2.13 PL spectra of $\mathrm{CsPbX}_{3}$ mixed halide NCs. . . . . . . . . . . . 71

2.14 TEM micrographs of $\mathrm{CsPbX}_{3}$ perovskite NWs. . . . . . . . . . . 72

2.15 XRD spectra of $\mathrm{CsPbX}_{3}$ NWs. . . . . . . . . . . . . 73

3.1 A schematic of LED fabricated in this work. . . . . . . . . . . . . 79

3.2 Perovskite LED structure with energy diagram and TEM image. . . 80

3.3 PL, EL, and photographs of peNC-LEDs. . . . . . . . . . . . . 84

3.4 Efficiency metric of peNC-LEDs. . . . . . . . . . . . . . . . . 85

3.5 PL reversibility test in peNC-LEDs. . . . . . . . . . . . . . 88

3.6 JV curve and EL peak movement in peNC-LEDs. . . . . . . . . . 89

3.7 Optical studies on mixed halide sample emitting at $566 \mathrm{~nm}$. . . . 90

3.8 Temperature dependent PL spectra of perovskite NCs. . . . . . . . 91

3.9 Voltage dependent EL analysis on peNC-LEDs. . . . . . . . . . . . 92

3.10 Voltage dependent EL studies on remaining samples. . . . . . . . . 94

3.11 Time dependent EL analysis on peNC-LEDs. . . . . . . . . . . . . . 95

3.12 A chart of peak wavelength vs time for peNC-LEDs. . . . . . . . . 96

3.13 Voltage dependency in peNC-LEDs. . . . . . . . . . . . . . . . 96

3.14 PL and EL analysis on O558 peNC-LED . . . . . . . . . . . . . . 99

3.15 Stability measurement of peNC-LEDs. . . . . . . . . . . . . . 102

4.1 Crystal structure of 2D and quasi 2D perovskite. . . . . . . . 106 
4.2 Device schematic and energy diagram of rpp-LEDs. . . . . . . . 112

4.3 TEM micrographs of RP perovskites nanosheets. . . . . . . . . . 113

4.4 High resolution TEM micrographs with crystal structure. . . . . . 115

4.5 X-ray diffraction spectra of RP perovskite nanosheets. . . . . . . 116

4.6 Absorption spectra of RP perovskite thin films. . . . . . . . . . . 118

4.7 Emission spectra of RP perovskite thin films. . . . . . . . . . . . 120

4.8 PL and EL spectra with photographs of rpp-LEDs. . . . . . . . . . 121

4.9 Efficiency metrics of blue-green LEDs with change in voltage. . . . 122

4.10 Efficiency metrics of blue-green LEDs with current density. . . . . 123

4.11 Efficiency metrics of red LEDs with change in voltage. . . . . . . . 124

$4.12 \mathrm{~J}-\mathrm{V}$ curve of $\mathrm{rpp}-\mathrm{LED} . \quad \ldots \ldots$. . . . . . . . . . . . . 127

4.13 EL spectra of $r p p-L E D s$ at varying voltages. . . . . . . . . . . . . . 128

4.14 Stability test on green rpp-LEDs. . . . . . . . . . . . . . . 130

4.15 CIE diagram of $\operatorname{rpp}-$ LEDs $\ldots \ldots \ldots$. . . . . . . . . 131

5.1 TEM micrographs of thallium lead iodide NWs with impurity. . . . 141

5.2 TEM micrographs of optimised $\mathrm{TlPbI}_{3} \mathrm{NWs}$. . . . . . . . . . . . 142

5.3 SEM and TEM images of $\mathrm{TlPbI}_{3}$ NWS with EDS map. . . . . . . . . 143

$5.4 \mathrm{XRD}$ of $\mathrm{TlPbI}_{3} \mathrm{NWs}$ with crystallography analysis. . . . . . . . . . . 144

5.5 TEM and XRD of $\mathrm{Tl}_{3} \mathrm{PbI}_{5} \mathrm{NCs} \ldots \ldots \ldots \ldots \ldots \ldots$

5.6 TEM and XRD of $\mathrm{Tl}_{3} \mathrm{PbBr}_{5}$ and $\mathrm{Tl}_{3} \mathrm{PbCl}_{5} \mathrm{NCs}$. . . . . . . . . 148

5.7 Crystallography analysis of $\mathrm{Tl}_{3} \mathrm{PbBr}_{5}$ and $\mathrm{Tl}_{3} \mathrm{PbCl}_{5}$ NCs. . . . . . . 148

5.8 TEM micrographs of size controlled $\mathrm{Tl}_{3} \mathrm{PbBr}_{5}$ NCs. . . . . . . . . 150

5.9 Particles size estimation for $\mathrm{Tl}_{3} \mathrm{PbBr}_{5}$ and $\mathrm{Tl}_{3} \mathrm{PCl}_{5}$ NCs. . . . . . . . 151

5.10 XRD comparison of mixed halide NCs to the theoretical pure halide.152

5.11 XRD comparison of mixed halide NCs with theoretical spectra. . . 153

5.12 Absorption spectra of NWs and NCs. . . . . . . . . . . . . . 157

5.13 Tauc plot of NWs and NCs. . . . . . . . . . . . . . . . . 158

5.14 Effect of composition on the direct band gap of $\mathrm{Tl}_{3} \mathrm{PbX}_{5} \mathrm{NCs} . . .160$

5.15 Emission spectra of $\mathrm{Tl}_{3} \mathrm{PbI}_{5}$ NCs. . . . . . . . . . . . . . 161

5.16 Electrical measurements on $\mathrm{TlPbI}_{3} \mathrm{NWs}$. . . . . . . . . . . . . 164 
7.1 A synthesis method of $\mathrm{CuInS}_{2}$ Ncs. . . . . . . . . . . . . . 175

7.2 TEM and XRD of CuInS ${ }_{2}$ NCS. . . . . . . . . . . . . . . . 177

7.3 Absorption spectra of $\mathrm{CuInS}_{2}$ NCs. . . . . . . . . . . . . . 178

7.4 SEM images of perovskite and perovskite/CuInS ${ }_{2}$ thin films. . . . 179

7.5 Performance of perovskite solar cells with schematic diagram . . . 180

7.6 Optical study on $\mathrm{Er}^{3+}$-doped $\mathrm{Tl}_{3} \mathrm{PbBr}_{5} \mathrm{NCs}$. . . . . . . . . . . 183

7.7 TEM analysis of $\mathrm{Er}^{3+}$-doped $\mathrm{Tl}_{3} \mathrm{PbBr}_{5} \mathrm{NCs} \ldots \ldots . \ldots 183$ 


\section{List of Tables}

2.1 Reaction conditions for $\mathrm{CsPbBr}_{3}$ NCs. . . . . . . . . . . . . 58

2.2 NCs size estimate and optical characterisation measurement data. 62

2.3 Loading ratio and PL for halide composition tuned NCs. . . . . . . 65

3.1 PL and EL metrics for champion devices. . . . . . . . . . . . . . 82

3.2 Device statistics on all of the fabricated LEDs at applied voltage. . 86

4.1 Precursor concentration for perovskites solution. . . . . . . . . . 111

4.2 LEDs statistics for rpp-LEDs . . . . . . . . . . . . . . . . . 125

4.3 A table for efficiency comparison of rpp-LEDs with literature. . . . 126

4.4 A table for power consumption in RP perovskite LEDs . . . . . . 127

5.1 Reaction conditions for thallium lead halide NCs and NWs. . . . . 139

5.2 Band gap of thallium lead halide NCs . . . . . . . . . . . . . . 159

5.3 A table for the fitting values . . . . . . . . . . . . . . . 162

7.1 Efficiency metrics of perovskite solar cells. . . . . . . . . . . 179 


\section{List of Abbreviations}

$\begin{array}{ll}B A & \text { Butylammonium } \\ C I S & \text { CuInS }_{2} \\ E L & \text { Electroluminescence } \\ E Q E & \text { External quantum efficiency } \\ F A & \text { Formamidinium } \\ G S B & \text { Ground state bleach } \\ I C P-M S & \text { Inductively coupled plasma mass spectrometry } \\ I T O & \text { Indium tin oxide } \\ L E D s & \text { Light emitting diodes } \\ M A & \text { Methylammonium } \\ N C s & \text { Nanocrystals } \\ N W s & \text { Nanowires } \\ P C E & \text { Power conversion efficiency } \\ p e N C-L E D s & \text { Perovskite nanocrystal LEDS } \\ P L & \text { Photoluminescence } \\ Q D s & \text { Quantum dots } \\ R P & \text { Ruddlesden-Popper } \\ r P p-L E D s & \text { Ruddlesden-Popper perovskite LEDs } \\ S E M & \text { Scanning electron microscopy } \\ T E M & \text { Transmission electron microscopy } \\ U V-V i s & \text { Ultraviolet-Visible } \\ X R D & \text { X-ray diffraction }\end{array}$




\section{Chapter 1}

\section{Background and Methods}

\subsection{Introduction}

This thesis presents the synthesis of perovskite semiconductor NCs (NCs) for functional optoelectronic devices, specifically light emitting diodes (LEDs). NCs were synthesised by colloidal chemistry and then processed to implement in solution processed LEDs. Additionally, Some nanostructured crystals were grown by in-situ solution processed technique on a substrate for the fabrication of highly efficient LEDs.

Semiconductor NCs are one of the most promising candidates for optoelectronic applications [1-5]. The optical and electrical properties of NCs differ from their bulk counterparts due to a small surface to volume ratio [6]. The optical properties of NCs can be tuned by changing the particle size. Hence, smaller particles have larger band gaps and larger particles have smaller band gaps [7]. The most promising approach to synthesise NCs is a colloidal chemistry using suitable ligands $[1,8]$. Particle size and shape can be controlled by changing the reaction condition such as precursor concentration, ligand concentration, reaction time, and temperature [8-10]. To date, CdS and CdSe NCs produce the most efficient LEDs $[1,11]$. Lead halide perovskite materials, which 
have $\mathrm{ABX}_{3}$ type crystal structure, have shown remarkable properties such as high absorption coefficient, high exciton binding energy, direct band gap, low defect density, and, most importantly, a halide composition dependent band gap [12-14]. That means the optical properties can be tuned by changing the ratio between halides in the perovskite structure. All of these properties are very useful for optoelectronic applications, such as solar cells, LEDs, photodetectors, and laser applications [14-16].

NCs of these materials are even more interesting as they combine the advantages of crystalline perovskite as well as QDs [17-19]. Inorganic metal halide perovskite $\mathrm{NCs}\left(\mathrm{CsPbX}_{3}, \mathrm{X}=\mathrm{Cl}, \mathrm{Br}, \mathrm{I}\right)$ have shown colour tuneability through out the entire visible spectrum by taking the advantage of quantum confinement as well as halide mixing [17]. In addition, they have also shown the PL quantum yields of $90 \%$, which is very competitive to the Cd based QDs [17]. Due to their high quantum yield and precise colour tuneability these nanomaterials are a promising candidate for LEDs application [20, 21]. Since the invention of $\mathrm{CsPbX}_{3} \mathrm{NCs}$ [17], researchers have gained interest in finding other perovskite or perovskite like NCs with useful properties by replacing A, B, and X groups from the perovskite crystal structure to find other feasible atoms $[8,22-$ 25]. However, NCs always suffer from poor conduction properties due to their bulky ligands. Ligand exchange can help to replace them with less insulating ligand but this process compromises the PL quantum yield, and device quality [26, 27]. Later on, a metal halide Ruddlesden-Popper phase perovskites have been discovered with a similar crystal structure. However, RP perovskites consist a number of perovskite monolayers together to form a thicker nanosheet [28-33]. Each nanosheet is separated by some organic cation to form a quasi$2 \mathrm{D}$ structure. These RP phase perovskite were found to be even more efficient than perovskite QDs for LEDs and solar cells application due to their high stability and improved conducting properties. [30, 32-34]. They combine the colour purity and tuneability of QDs with the conduction properties of 3D crystals [35-37]. 
Designing the device structure, optimising the material properties, and processing of these NCs for practical device applications are very challenging, and require a lot of optimisation. This thesis includes the synthesis of these NCs with the optimisation to process them for device quality thin films followed by the fabrication of efficient LEDs emitting through the entire visible region.

This chapter contains the basic synthesis methods and mechanisms of NCs by colloidal chemistry, properties of NCs, and the confinement effect which relates to the density of states. In addition, the second half of the chapter will introduce the metal halide perovskite crystals and their advantages followed by the history and working mechanism of LEDs. Afterwards, application of perovskites in solar cells is discussed. The last part of this chapter will contain the methods section including the major characterisation techniques used in this project.

Chapter 2 contains the synthesis of size and composition controlled $\mathrm{CsPbX}_{3}$ NCs with tuneable optical properties. For the first time, the evolution of quantum confinement was conducted for the size controlled $\mathrm{CsPbBr}_{3} \mathrm{NCs}$, which suggest the strong confinement in these NCs [38]. In addition, halide composition was varied to synthesise the NCs with precise colour tuneability through the entire visible spectrum. These NCs were used in Chapter 3 for the fabrication of LEDs. Those LEDs showed an EL through the entire visible spectrum, specifically focused on the red spectral region. For the first time, the observation and mechanism of colour instability and ion migration in mixed halide perovskite NC red LEDs was clearly conducted [20]. Chapter 4 contains the synthesis of pure inorganic Ruddlesden-Popper (RP) phase perovskite structures, which shows the conduction properties similar to 3D perovskites while having confinement properties similar to NCs. These RP phase perovskite were used for the fabrication of LEDs emitting in the entire visible spectrum. The halide mixing in RP phase perovskite was performed for the first time. In addition, the EQE of the blue LEDs outperformed the current literature values, and showed very competitive EQE in the green emission region along with a 
relatively long life time [30, 39]. After this work, we were interested in finding novel perovskite NCs by replacing group A in the perovskite crystal structure. We developed shape-, size-, and composition-controlled thallium lead halide nanowires (NWs) and NCs in Chapter 5 [8]. The size tuning as well as composition tuning was demonstrated in these NCs. In addition, we used $\mathrm{TlPbI}_{3} \mathrm{NCs}$ as a wide band gap photoconductor. These novel perovskite NCs have potential applications in solar cells, X-ray detectors, piezoelectrics, and non-linear optics when doped with rare earth material.

\subsection{QDs}

Semiconductor NCs that are small enough to exhibit zero dimensional (atomiclike) quantum mechanical properties are called QDs. Alexei Ekimov synthesised QDs in a glass matrix in the beginning of the 1980s [40]. While Louis E Brus discovered a means of producing QDs in colloidal solutions [41, 42]. The size of QD range from 2-50 $\mathrm{nm}$ in diameter, and usually contains 102-104 atoms. The properties of QDs, such as optical and electrical, depend on their shapes and sizes. In addition, shape and size of QDs can be tuned by changing the reaction conditions.

\subsubsection{History}

The semiconductor colloids were first developed using the arrest precipitation method. [43]. In this method, poorly soluble cations and anions tend to precipitate in aqueous solution when they are at high concentration of supersaturation. For example, metal salts of $\mathrm{Cd}\left(\mathrm{SO}_{4}\right)_{2}$ and $\left(\mathrm{NH}_{4}\right)_{2} \mathrm{~S}$ in an aqueous solution precipitate at reaction equilibrium, which results in the formation of CdS precipitate (Equation 1.1). 


$$
\left.C d_{(a q)}^{2+}+S_{(a q)}^{2-} \longleftrightarrow C d S_{(} s\right)
$$

The formation of a bright yellow colour in this reaction confirmed the formation of CdS colloids. The band gap of this material matched with the bulk band gap of CdS, however, there was no indication of confinement effect, which is usually observed by the presence of a high energy absorption peaks [43]. A similar method was used to produce semiconductor colloids of $\mathrm{ZnS}$ in methanol and water [44]. The rate of precipitation was found to be rapid in these reactions and therefore some polymer additives were used to preclude fast precipitation $[43,45-48]$. While this method was capable of creating various NCs, the PL quantum yield (emission) was very low with broad spectral emission due to the surface defects resulting in non-radiative decay path ways [49]. In addition, this method was very limited to control the size of NCs [49]. Significant improvement was made to control the size and which offered narrow size distribution [50]. However, a new synthesis method for high quality NCs was needed.

Templated growth of NCs in micelles or reverse micelles have been used. A micelle is a surfactant molecule, which has a hydrophilic head and a hydrophobic tail. A micelle is formed when the hydrophilic head is exposed to the surrounding aqueous solvent as shown in Figure 1.1. On the other hand, if the hydrophobic part is in the contact with surrounding non-aqueous solvent, then it forms a reverse micelle [51]. Mauricio was the first one to synthesise CdS NCs in micelles [52]. Several other semiconductor NCs were synthesised using micelle or reverse micelle such as CdTe [53], CdSe [53], ZnS [54], ZnSe [55], PbS [56], HgSe [53], and HgS [57]. These micelle materials offer a superior size control and distribution than the arrested precipitation method. However, the PL spectra of NCs, synthesied by this method, still displayed a weak broad emission. Control over size and size distribution allowed researchers to understand the photophysics in these NCs more systematically. Due to the high surface area in NCs, there are more structural defects which results in charge trapping in those de- 
fects site [49]. The enhancement in the PL quantum yield was performed by passivating the surface of CdSe NCs by a higher band gap shell, which resulted in electron-hole overlap, and thus radiative recombination of the electron and the hole [54]. This results in the increase of PL quantum yield with a thin emission line width [54].

Another method to synthesise NCs was the organometallic synthesis, which uses an organometallic precursor. These precursors, such as hydrogen chalcogenides $\left(\mathrm{H}_{2} \mathrm{~S}, \mathrm{H}_{2} \mathrm{Te}, \mathrm{H}_{2} \mathrm{Te}\right)$ were used in arrested precipitation rather than ionic sulfur $\left(\mathrm{Na}_{2} \mathrm{~S}\right)$ [58-60]. The advantage of using these precursors was better size control and distribution. Later on, an anion precursor bis(trimethylsilyl) selenide was widely used for the synthesis of NCs [53]. Thus, the anionic precursor was replaced by bis(trimethylsilyl) selenide and hydrogen chacogenide $\left(\mathrm{H}_{2} \mathrm{~S}\right.$, $\mathrm{H}_{2} \mathrm{Te}, \mathrm{H}_{2} \mathrm{Te}$ ). Steigerwald et al. used tertiary phosphine chalcogenides, triethylphosphine telluride, to synthesise solution processed HgTe [61]. After the successful synthesis of HgTe, researchers used the similar tertiary phosphine

a) Micelle

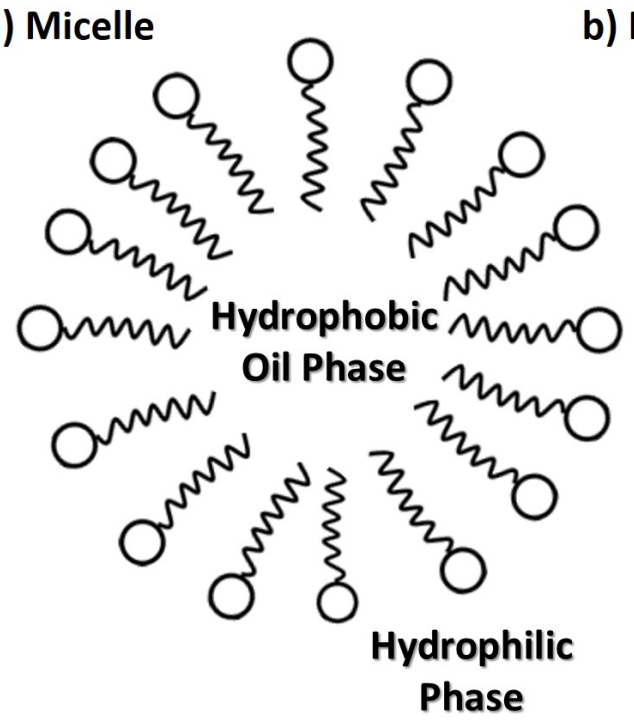

b) Reverse Micelle

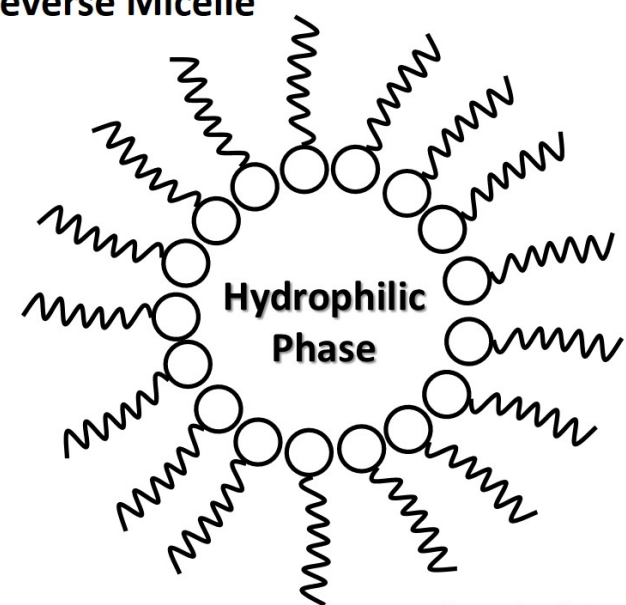

Hydrophobic Oil Phase

Figure 1.1: a) Micelle and b) reverse micelle structure have a hydrophobic tail and a hydrophilic head. 
chalcogenide in synthesis of NiTe [62], CoTe [63], CoSe [64], CoS [64], MnTe [65], PdTe [66], and FeTe [67]. Thus the use of different precursor solutions, ligands, and core/shell structures evolved the variety of semiconductor NCs.

\subsubsection{Shape Control of NCs}

NCs can be grown in various shapes such as rods, wires, or a disk-like shape. A hexagonal and wurtzite structure exhibit different facets and these facets can show different chemical reactivity. Due to this, some precursors react with one facet more quickly than the other. Therefore, the NCs growth is thermodynamically favoured along that facet. The growth of wurtzite CdSe was found to be favorable along the most polar axis when the growth of NCs is fast [9]. Peng et al. reported a clear mechanism for anisotropic growth, where they suggested that a slower growth rate can be achieved by choosing a longer alkyl chain ligand [9]. Interestingly, this very slow growth rate results in anisotropic growth as the crystal has the highest reactivity perpendicular to the c axis (001) in a wurtzite crystal of CdSe [9]. In addition, the growth rate was also found to be dependent on the precursor concentrations: a higher concentration of precursor led to an anisotropic growth [9]. Various shape of NCs for example, Co NCs with disk shape,[68] and PbS NCs with cubic shape,[69] could be synthesised. These shapes were controlled by the same facts that NCs show more favorable growth on one particular facet than others, as most of the NCs exhibit faceted shape,[70] and this can be achieved by varying the precursor concentrations, reaction conditions, and the type of ligands. 


\subsubsection{Density of States}

The density of states (DOS) is the number of energy state per unit energy in a given volume. In order to calculate the density of states in 3-dimensional semiconductors, an infinite quantum well is assumed, where the electron has free movement. The semiconductor is assumed to be a perfect cube with a length L (Figure 1.2a). The wave function in this condition will be the following:

$$
\psi=A \sin \left(K_{x}\right)+B \cos \left(K_{x}\right)
$$

Where $\mathrm{A}$ and $\mathrm{B}$ are constants and their values are dependent on the boundary conditions. One of the boundary condition states that upon reaching the edge of the crystal, the wave function decreases to zero. That means $\mathrm{k}_{x}$ should
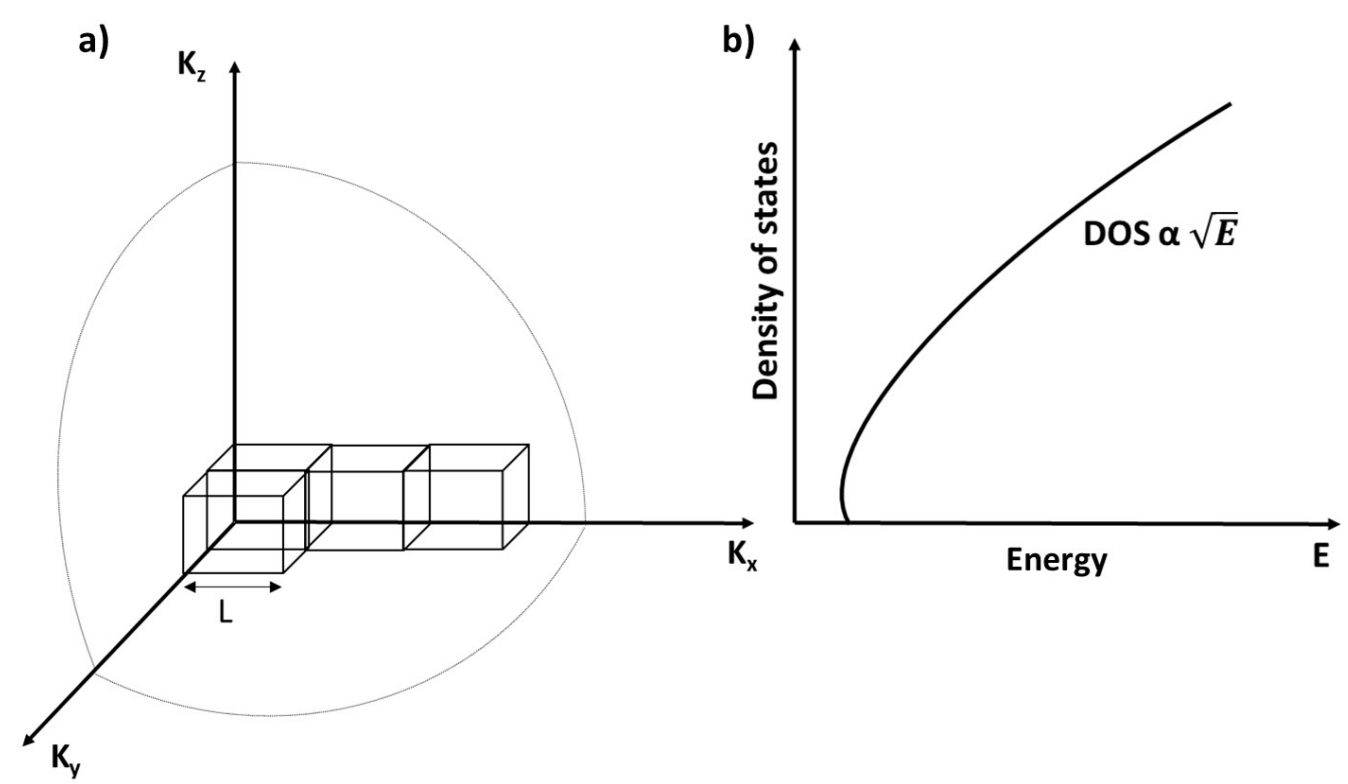

Figure 1.2: a) A diagram for the calculation of density of states in a 3dimensional semiconductor. b) Density of state shows the parabolic relationship with energy, which is observed from Equation 1.19. 
be the integer multipal of $\pi$.

$$
k=\frac{m \pi}{L}, m=0,1,2 \ldots
$$

All of these $\mathrm{k}$ values can also be represented in $\mathrm{y}$ and $\mathrm{z}$ direction. Since the crystal is isotropic with equal length (L) in all directions, the volume for each of these states in $\mathrm{k}$ space will be the following:

$$
V=\left(\frac{\pi}{L}\right)^{3}
$$

Now, we calculate the number of states $(\mathrm{N})$ in the given value of $\mathrm{k}$ space (Figure 1.2a). This will be the fraction between the volume of an eighth of a sphere with radius $\mathrm{k}$ and the volume of a single cube calculated in Equation 1.4. Each of these states could be occupied by spin up and spin down hence the factor of 2 will be multiplied.

$$
\begin{gathered}
N=2 \frac{1}{8}\left(\frac{4}{3} \pi k^{3}\right)\left(\frac{L}{\pi}\right)^{3} \\
N=\frac{\pi k^{3}}{3}\left(\frac{L}{\pi}\right)^{3}
\end{gathered}
$$

Differential with respect to k of Equation 1.6 will be the following:

$$
d N / d k=3 \pi k^{2}\left(\frac{L}{\pi}\right)^{3}
$$

As the density of states is defined as the number of states per unit energy in a given volume $(\mathrm{V})$ therefore, the density of states is defined by the following formula:

$$
\begin{aligned}
& D O S=\frac{d N}{d E} \frac{1}{V} \\
& D O S=\frac{d N}{d E} \frac{1}{L^{3}}
\end{aligned}
$$

Equation 1.9 can also be written in the form of wavenumber $\mathrm{k}$ : 


$$
D O S=\left(\frac{d N}{d k}\right)\left(\frac{d k}{d E}\right)\left(\frac{1}{L^{3}}\right)
$$

By taking the value of $\mathrm{dN} / \mathrm{dK}$ from Equation 1.7, the above equation becomes:

$$
\text { DOS }=\frac{k^{2}}{\pi^{2}} \frac{d k}{d E}
$$

The relation between the kinetic energy and the wavenumber of a particle with given mass $\mathrm{m}^{*}$ is given as [71]:

$$
E=\frac{\hbar^{2} k^{2}}{2 m^{*}}
$$

Where $\hbar=\frac{h}{2 \pi}, \hbar$ is called the reduced Planck constant and $\mathrm{h}$ is the Planck constant with value $6.62607004 \times 10^{-34} \mathrm{~m}^{2} \mathrm{~kg} / \mathrm{s}$.

$$
\begin{aligned}
& \frac{d E}{d k}=\frac{\hbar^{2} k}{m^{*}} \\
& \frac{d k}{d E}=\frac{m^{*}}{\hbar^{2} k}
\end{aligned}
$$

From Equation 1.12, we can extract the value of wavenumber $\mathrm{k}$.

$$
k=\frac{\sqrt{2 m^{*} E}}{\hbar}
$$

By taking the value of $\mathrm{dk} / \mathrm{dE}$ from Equation 1.14 and putting this in Equation 1.12, the density of states becomes:

$$
\begin{gathered}
D O S=\frac{k m^{*}}{\hbar^{2} \pi^{2}} \\
D O S=(E)^{(1 / 2)} m^{*(3 / 2)} \frac{8 \pi \sqrt{2}}{h^{3}}, \hbar=\frac{h}{2 \pi}
\end{gathered}
$$

Equation 1.17 shows that the density of states is proportional to the square root of energy.

$$
D O S \propto E^{(1 / 2)}
$$




$$
(D O S)^{2} \propto E
$$

Equation 1.19 shows the parabolic relationship $\left(y^{2}=4 \mathrm{ax}\right)$ between the density of states and energy. Therefore, the energy diagram shows the continuous parabolic dispersion (figure $1.2 \mathrm{~b}$ ).

Similarly, the density of state can be calculated in 2-dimensional semiconductors such as NWs and nanorods. In a 2-dimensional system, the carriers are confined in the $\mathrm{z}$ direction. Therefore, we only have a 2-dimensional $\mathrm{k}$ space (Figure 1.3a) and each of these states occur at a distance of $\frac{\pi}{L}$ from Equation 1.3. Thus, the area of the square can be described as:

$$
A=\left(\frac{\pi}{L}\right)^{2}
$$

Similar to Equation 1.6, the number of states in the given volume of $\mathrm{k}$ space (Figure 1.3a) will be the fraction between the area of a fourth of a circle with radius $k$ and the area of a single square. Each of these states could be occupied by spin up and spin down hence the factor of 2 will multiplied.

$$
N=\frac{k^{2} L^{2}}{2 \pi}
$$

Differential of the above equation with respect to wavenumber $\mathrm{k}$ will be:

$$
\frac{d N}{d K}=\frac{k L^{2}}{\pi}
$$

As the density of states is the number of states per unit energy in a given volume, therefore similar to Equation 1.8, it can be written as:

$$
\operatorname{DOS}_{2 D}=\frac{d N}{d K} \frac{d K}{d E} \frac{1}{A}
$$



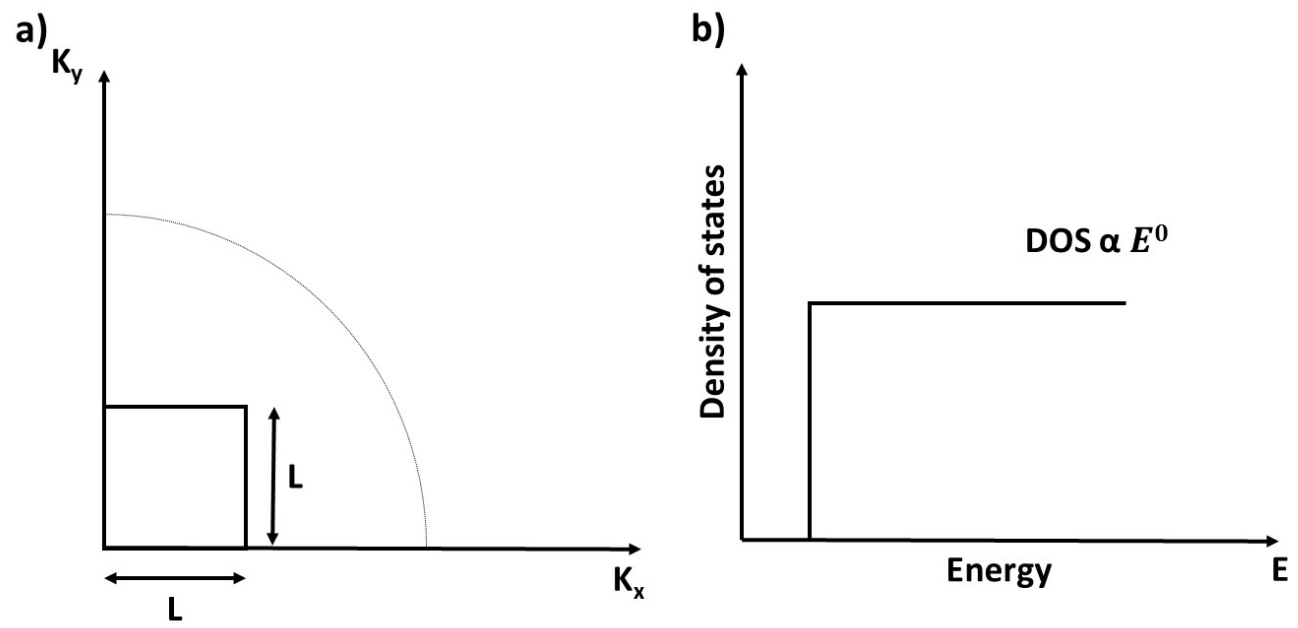

Figure 1.3: a) A diagram for the calculation of density of state in a 2 dimensional semiconductor, such as NWs and nanorods. b) Density of state has no relationship with the energy, which was observed from Equation 1.25.

The similar strategies were used to derive the DOS in 2-dimensional semiconductors. The value of $\frac{d k}{d E}$ can be extracted from Equation 1.14. By putting all these values into Equation 1.23 and solving them, the DOS in two-dimensional semiconductor will be following:

$$
\operatorname{DOS}_{2 D}=\frac{4 \pi m^{*}}{h^{2}}
$$

Where $\mathrm{m}^{*}$ is the mass of the particle. As can be seen from Equation 1.24, the density of states in 2-dimensional semiconductor does not depend on energy at all (Figure 1.3b). So, the above Equation can be written in the following form:

$$
D O S_{2 D} \propto E^{0}
$$

Similarly, in a 1-dimensional semiconductors the carriers will be confined in two dimensions and will only be free to move in one dimension. Therefore, using the same strategies as used in the three and two-dimensional semicon- 
ductors, the electron will be free to move only in one dimensional $\mathrm{k}$ space as shown in $1.4 \mathrm{a}$ and they are separated by a distance $\frac{\pi}{/} L$. In this case the total number of states $(\mathrm{N})$ will be the fraction between total length $\mathrm{k}$ and $\frac{\pi}{l} L$. Each of these states could be occupied by spin up and spin down hence the factor of two will be multiplied as well. Thus, the total number of states will be following:

$$
N=2 k\left(\frac{L}{\pi}\right)
$$

Differential of the above equation with respect to wavenumber $\mathrm{k}$ will give the following solution:

$$
\frac{d N}{d K}=2\left(\frac{L}{\pi}\right)
$$

The density of states is the number of states per unit energy in a given volume (2 dimensions are confined). Therefore, the density of states in one-dimensional semiconductors will be:

$$
\operatorname{DOS}_{1 D}=\frac{d N}{d k} \frac{d k}{/} d E \frac{1}{L}
$$

The value of $\frac{d k}{d E}$ can be extracted from Equation 1.14 so the overall DOS in onedimensional semiconductor will be as following:

$$
\operatorname{DOS}_{1 D}=2 \sqrt{2} m^{(1 / 2)} E^{(-1 / 2)}
$$

Thus, in the case of 1-dimensional semiconductors, density of state is inversely proportional to the square root of energy, which can also be observed in Figure 1.4 . 

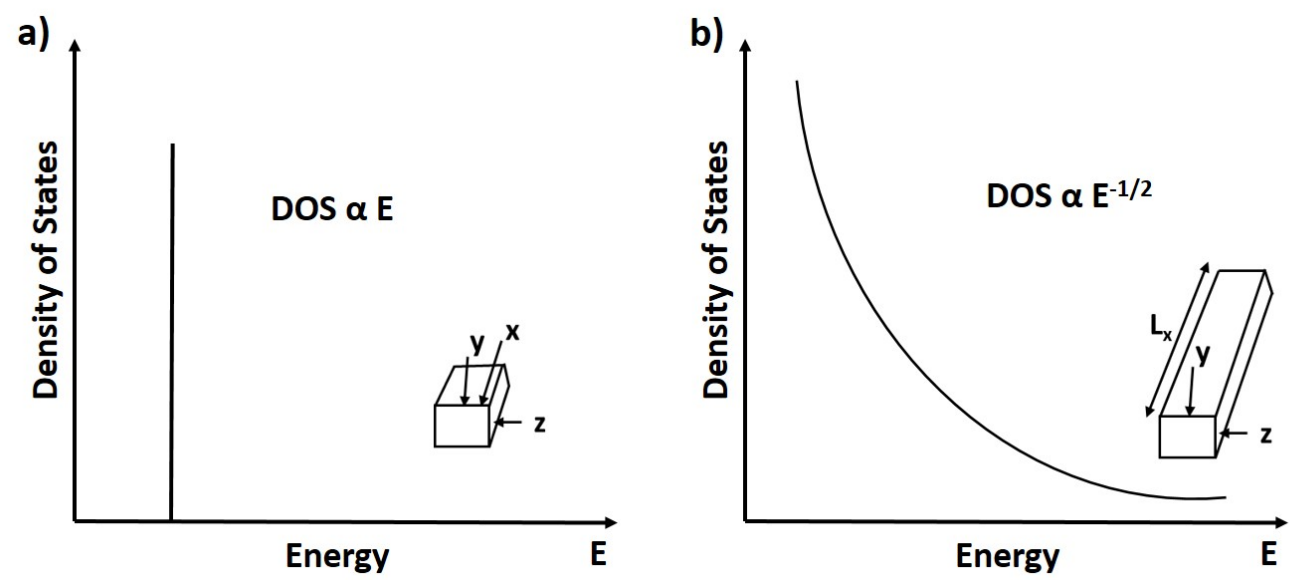

Figure 1.4: A relation between density of states and energy in a) 0-dimensional and b) 1-dimensional semiconductor. In the case of 1-dimensional semiconductor the DOS has the inverse relationship with energy. The zero dimensional semiconductor shows the direct proportion relation with energy.

$$
\operatorname{DOS}_{1 D} \propto \frac{1}{\sqrt{E}}
$$

In zero-dimensional semiconductors, QDs, the carriers will be confined in all of the dimensions. Therefore, $\mathrm{k}$ space will not be applied here. However, it is similar to single atom which will have discrete states. So the density of states will be written in a $\delta$ function.

$$
D O S_{0 D}=2 \delta E
$$

The Equation 1.31 shows that the density of states in zero-dimensional semiconductors will be directly proportional to the energy.

$$
D O S_{0 D} \propto E
$$

Equation 1.32 suggests that the energy level becomes discrete in the case of 
zero-dimensional semiconductors such as QDs. Thus, the overall derivation for 3-dimensional, 2-dimensional, 1-dimensional, and 0-dimensional semiconductors describes the relationship between density of states and energy.

\subsubsection{Band Gap Tuning}

As observed in Section 1.2.3, QDs have discrete energy levels and the band gap can be tuned by changing the size of the NCs which changes the degree of confinement. Any excitation source such as a photon is able to excite the electron from the valence band of a semiconductor material to conduction band. This process creates a hole in the valence band, which forms a bound pair of electron and hole that is called an exciton. The physical distance between the electron and the hole is called the Bohr radius of the exciton. Bohr radius is the radius of exciton quasiparticles, which is unique for each material. For a bulk material, this exciton Bohr radius is much smaller than the size of a particle so the radius can extend to its full natural limit. While, in the case of QDs, the exciton Bohr radius is either close to or larger than the particle size. NCs are considered to be in the weak confinement regime if the particle radius is larger than the Bohr radius. However, they are in the intermediate confinement regime, if the Bohr radius is close to the particle radius. Whereas, if the particle radius is smaller than the Bohr radius, then NCs are considered to be in the strong confinement regime. Therefore, excited electrons are unable to move to their full radius because they can not exist outside the QDs [72]. This effect is called as quantum confinement [72].

Band gap of NCs can be tuned by three possible ways. First method to tune the band gap is by tuning the size of NCs; the second method is by tuning the shape of NCs; and the last option is by synthesising alloy structures, in which the band gap can be tuned by changing the composition. 


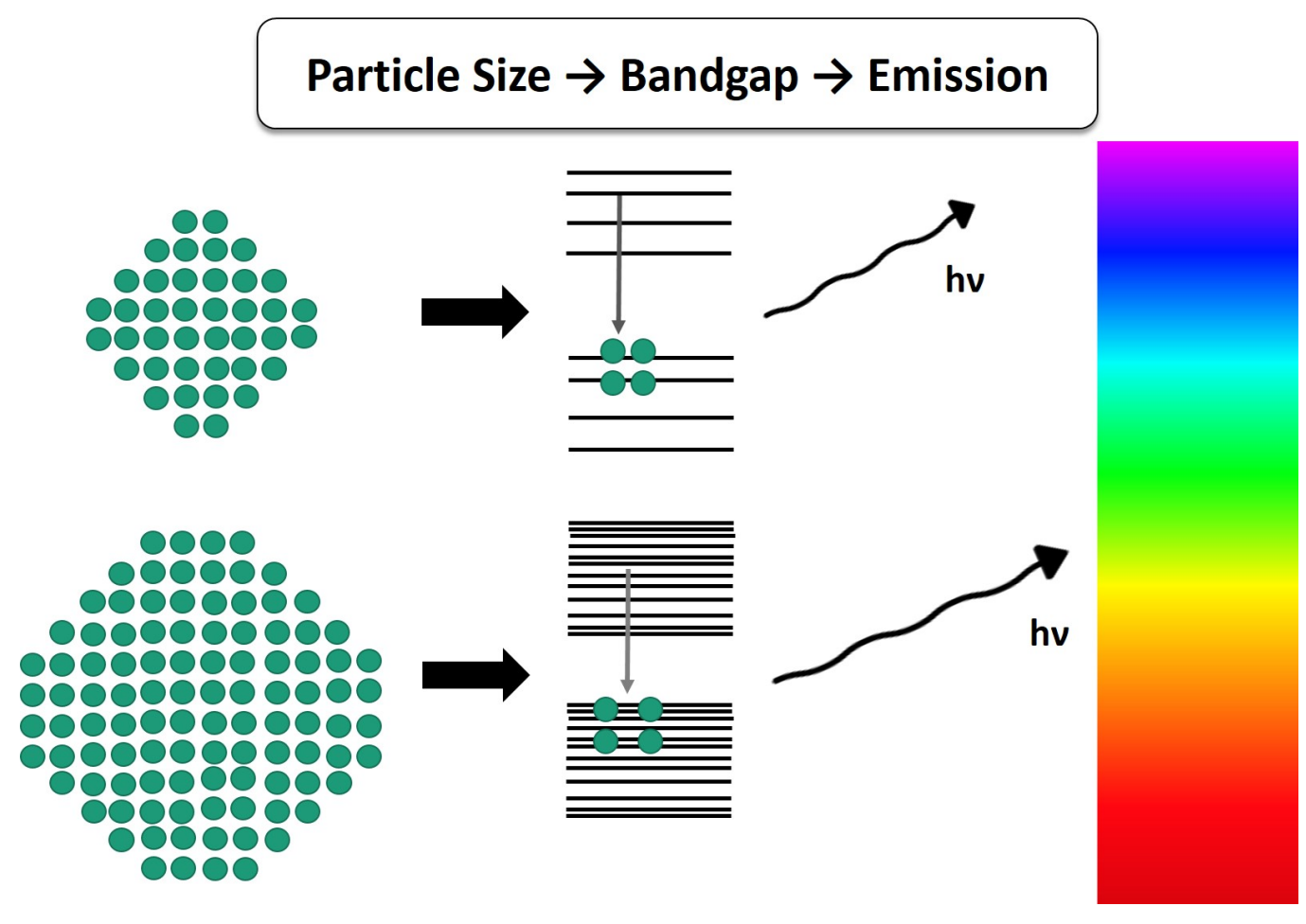

Figure 1.5: A diagram shows the dependence of particle size on band gap of NCs. Smaller particles result in a larger band gap and a blue shifted emission peak.

\subsubsection{Size Tuning}

Figure 1.5 shows the effect of the particle size on the band gap and thus the emission of NCs. When an electron of any atom is excited by an external light source, it absorbs energy and reaches the excited state (conduction band). After some time, the electron returns to its ground state (valence band) and emits a photon (in case of radiative recombination) with energy equal to the band gap of material. Thus, the band gap of QDs can be tailored by changing the particle size, which allows us to change the colour of emitted photons. Increase in the size of NCs results in a smaller band gap, whereas, the decrease in NCs size is responsible for the larger band gap of the material and therefore the emission shifts towards bluer wavelength as the size of NCs decreases [72, 73]. For instance, band gap tuneable size controlled CdSe NCs were synthesised by vary- 
ing the reaction conditions [74]. Similarly, size tuneable InP/ZnS QDs were synthesised by colloidal route with an emission from green to red spectral region $(510-630 \mathrm{~nm})[75]$.

\subsubsection{Shape Tuning}

The band gaps of nanomaterials can also be tuned by changing the band alignment and shape. This can be done by over coating a shell of other material or using a different ligand. Nanocrytsals can be achieved in a variety of shapes. For example, 0-dimensional NCs can be synthesised in cubic, spherical, and star shapes. 1-dimensional NCs can be synthesised as nanorods and NWs. Similarly, two-dimensional NCs can be made as disks and platelets [76]. Different shapes of NCs result in different band levels, which lead to changes in the band gap and therefore the emission peak.

\subsubsection{Alloy Structure}

Another method to tune the band gap is using alloy structure. A composition tuneability can lead to the tuneable band gap. For example, the band gap tuning in $\mathrm{Zn}_{x} \mathrm{Cd}_{1-x}$ Se NCs alloys was achieved by varying the amount of $\mathrm{Zn}$ and Se [77]. An increase in $\mathrm{Zn}$ content appeared to increase the band gap of the material. Thus, the emission spectra was tuned from 460 to $640 \mathrm{~nm}$ by varying the composition in $\mathrm{Zn}_{x} \mathrm{Cd}_{1-x}$ Se NCs [77]. Similarly, the band gap can be tuned by mixing the halides in metal halide perovskite crystals as well. Incorporation of chlorine results in a smaller band gap. However, incorporation of bromine and iodine leads to a larger band gap. This halide composition tuneability can shift the emission peak from blue to red spectral region [17]. The band gap tuning by halide mixing in perovskites crystals will be investigated in this thesis. 


\subsubsection{Colloidal Synthesis Method for NCs}

Colloidal synthesis of NCs can be explained using the LaMer model [78]. According to this model, there is a formation of monomers after the reaction precursors are dissolved in the solvent. The next step is the formation of nuclei, which can only occur when the concentration has reached the minimum concentration required for nucleation $\left(\mathrm{C}_{\text {min }}\right)$. As seen in the Figure 1.6 that the nucleation occurs by the aggregation of monomers and it stops when the concentration reaches below the minimum concentration $\left(\mathrm{C}_{\min }\right)$ required for nucleation (III). Below that concentration, the growth of NCs continues by one of these possible ways: a) addition of monomer, where the monomer deposits on to the nuclei; b) by Ostwald ripening, where smaller nuclei redissolve and deposit onto more thermodynamically favourable large nuclei; or c) by coalescence, where multiple NCs combine together and fuse to form a bigger NC [78]. The colloidal NCs can be synthesised in two most promising ways described below.

\subsubsection{Hot-Injection Technique}

In this technique, a room temperature precursor solution is injected into hot reaction medium in the presence of suitable surfactant molecules. Just after the rapid injection of precursor solution, the reaction becomes supersaturated and causes a sudden burst in the nucleation of QDs. When the room temperature precursor solution is added, the reaction temperature decreases and this precursor solution is coupled with the remaining precursor molecule thus further nucleation is prevented. Eventually, the growth of NCs occurs at lower temperatures than the temperature required for nucleation process. Thus, the growth of each nuclei is consistent for a very short period of time. This consecutive separation of the nucleation and growth processes generally allows a precise control of the size and shape of the QDs [79]. All perovskite NCs in this research were synthesised using the hot-injection technique. 


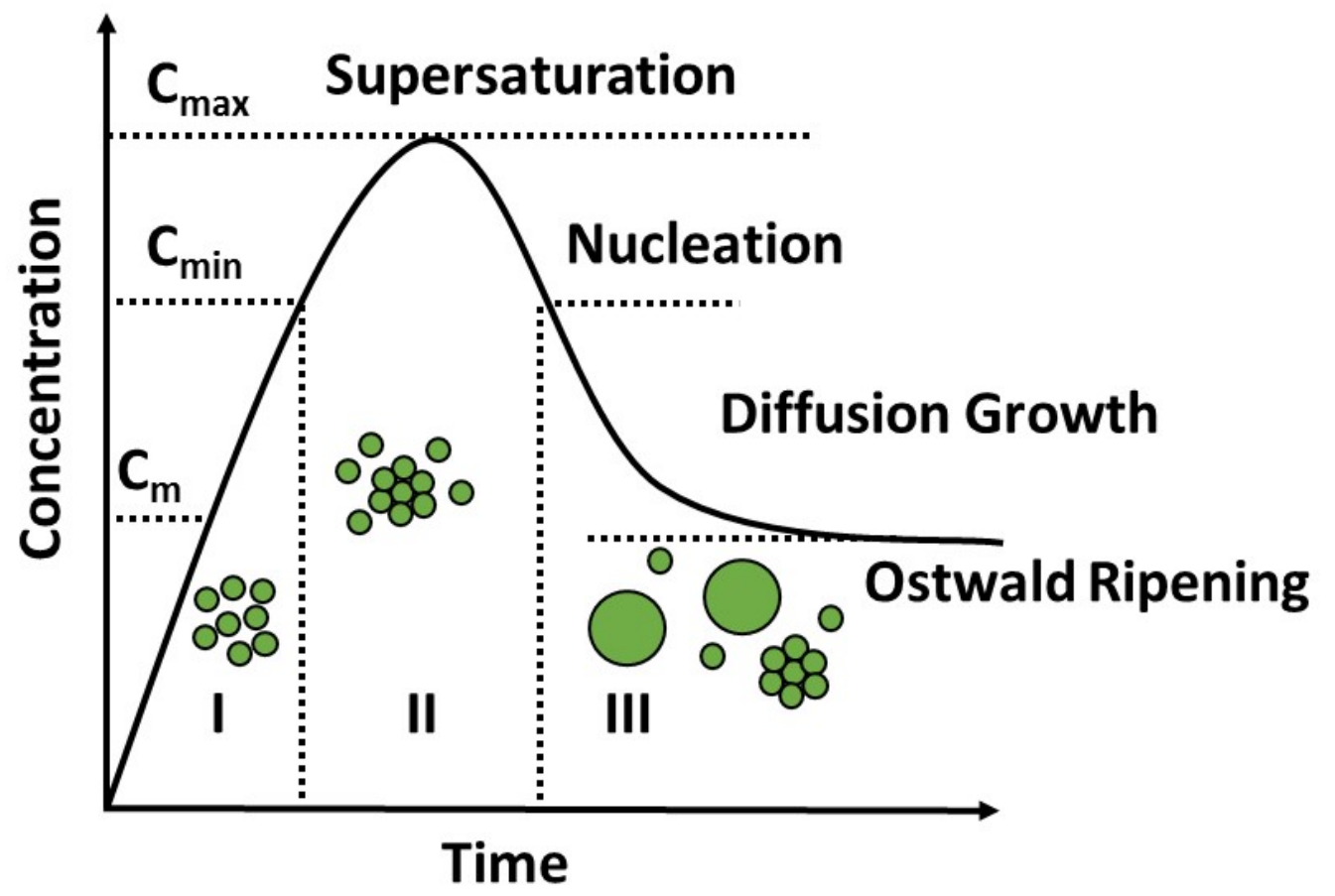

Figure 1.6: LaMer diagram describing the generation of atoms, nucleation and growth of NCs.

\subsubsection{Single-Pot Synthesis}

In this technique, all of the required precursors are mixed at room temperature and the system is heated to the suitable growth temperature. Unlike the hot-injection method, there is no supersaturation state. In this technique the level of supersaturation and the temperature of solution increases together and the nucleation rate depends on both. Using this process NCs can be made in large quantities with good reproducibility [79]. This method is widely used in industries. CuInS 2 NCs were synthesised by this method for the application in perovskite solar cells, which is described in Appendix-1. 


\subsubsection{Purification of NCs}

After the synthesis, NCs are purified from their growth solution to remove excess ligands. This can be done by mixing them in solvent and anti-solvent. The antisolvent mixes with the solvent but does not mix with the particles. The entire solution is centrifuged at certain RPM. Then, the supernatant is discarded while the precipitate is redissolved in solvent and antisolvent is added to crash out the particles. This process is conducted 2-4 times depending on the material. Eventually, the final precipitate of NCs is dispersed in solvent and used for further applications or characterisation. NCs were purified by the same purification method in this work.

\subsection{Metal Halide Perovskite}

A perovskite is a mineral of calcium titanium oxide $\left(\mathrm{CaTiO}_{3}\right)$, first discovered in Russia by Gustav Rose in 1839. Any mineral, which has a similar crystal structure, is called a perovskite structure. Perovskite was first investigated in the form of oxides [80]. However, we are mainly interested in metal halide perovskites for optoelectronic applications.

The standard chemical formula of a perovskite is $\mathrm{ABX}_{3}$, where $\mathrm{A}$ is a monovalent cation and $\mathrm{B}$ is a divalent cation, while $\mathrm{X}$ is an anion. Figure 1.7 shows the general structure of a halide perovskite crystal. Atoms of element A are situated on the corner of a cubic cell, atoms of element B (grey) are in the center and atoms of element $\mathrm{X}$ (violet) are in the center of each face. The band gap of metal halide perovskites can be tuned by varying the identity of the anions and cations. For example, by changing the A, B or X group element, it is possible to tune the band gap of the material. Chloride perovskites tend to have a larger band gap in comparison to iodide perovskites. Thus, by mixing these halides, the band gap can be engineered between their parent materials. 


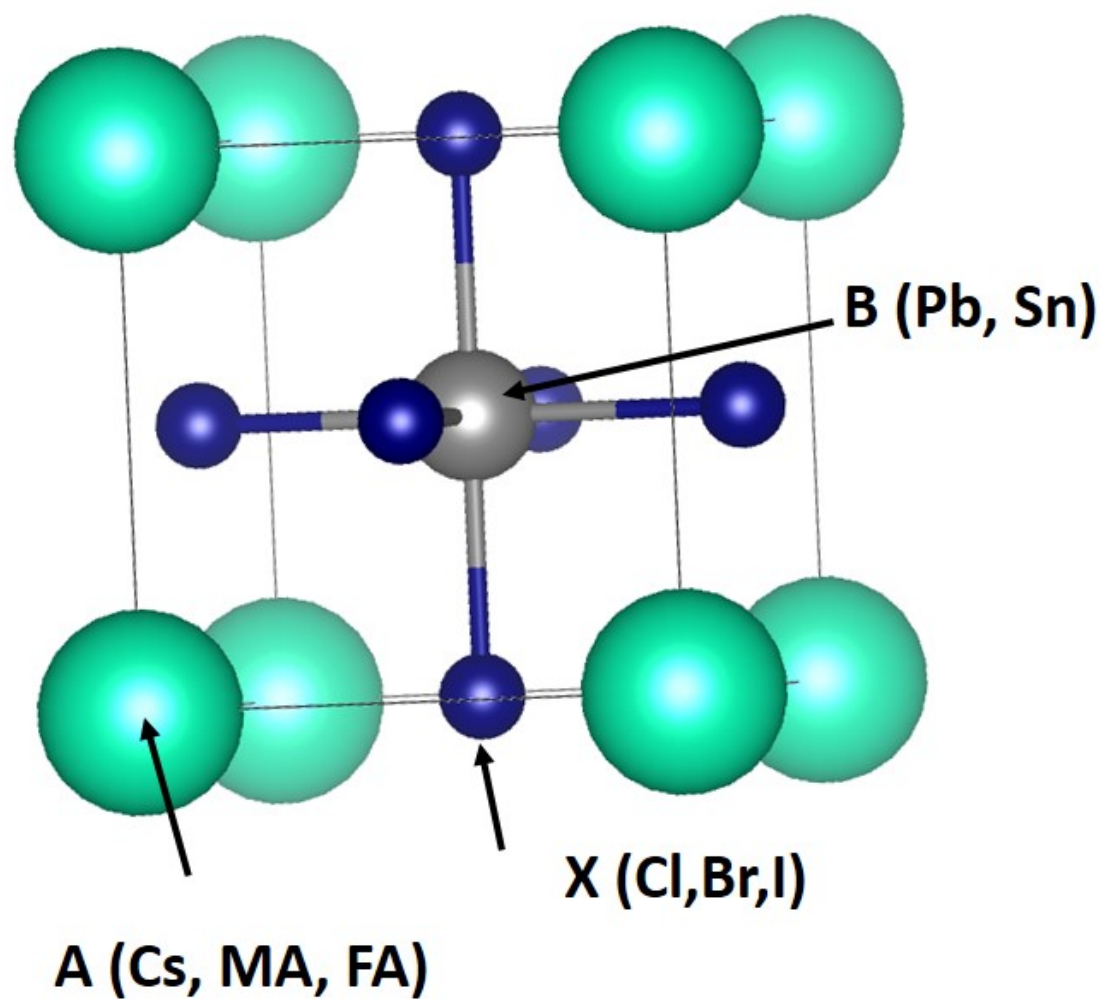

Figure 1.7: A halide perovskite crystal structure. Atom A is a monovalent cation, $B$ is a divalent cation, and $X$ is a halogen. Any crystal similar to this structure is called a perovskite crystal structure.

These materials are considered to be promising for optoelectronic applications because of their high absorption coefficient, tuneable band gap, long charge carrier lifetimes, charge diffusion lengths, [81, 82] and low defect densities [83]. Low cost photovoltaic devices made from these materials display high power conversion efficiencies (PCEs) and external quantum efficiencies (EQE) [84]. Recently, 70 \% PL quantum yield has been recorded from $\mathrm{CH}_{3} \mathrm{NH}_{3} \mathrm{PbI}_{3}$ and it was used in perovskite light-emitting diodes (pe-LEDs) [85]. 


\subsubsection{Perovskite NCs}

Bulk perovskites display relatively wide emission spectra and high quantum yields, which can only be achieved at high excitation density. They were also found to be unstable in ambient conditions. In addition, the colour tuneability in bulk perovskite is limited to the halide composition only [86, 87]. To overcome all of these issues, perovskite NCs have gained significant attention, as they combine the advantage of crystalline perovskite as well as those of NCs. Therefore, perovskite NCs offer better control over optical and electrical properties by both halide composition tuning and confinement effect. A thin emission line width with precise colour tuneability and high quantum yield could be achieved in perovskite NCs [17, 19, 88, 89].

Schmidt et al. were the first to synthesise $6 \mathrm{~nm}$ sized $\mathrm{CH}_{3} \mathrm{NH}_{3} \mathrm{PbBr}_{3} \mathrm{NCs}$ with 20 $\%$ PL quantum yield [88]. Hassan et al. reported the synthesis of $\mathrm{CH}_{3} \mathrm{NH}_{3} \mathrm{PbBr}_{3}$

\section{$\mathrm{CsPb}_{3} \mathrm{NCs}$}

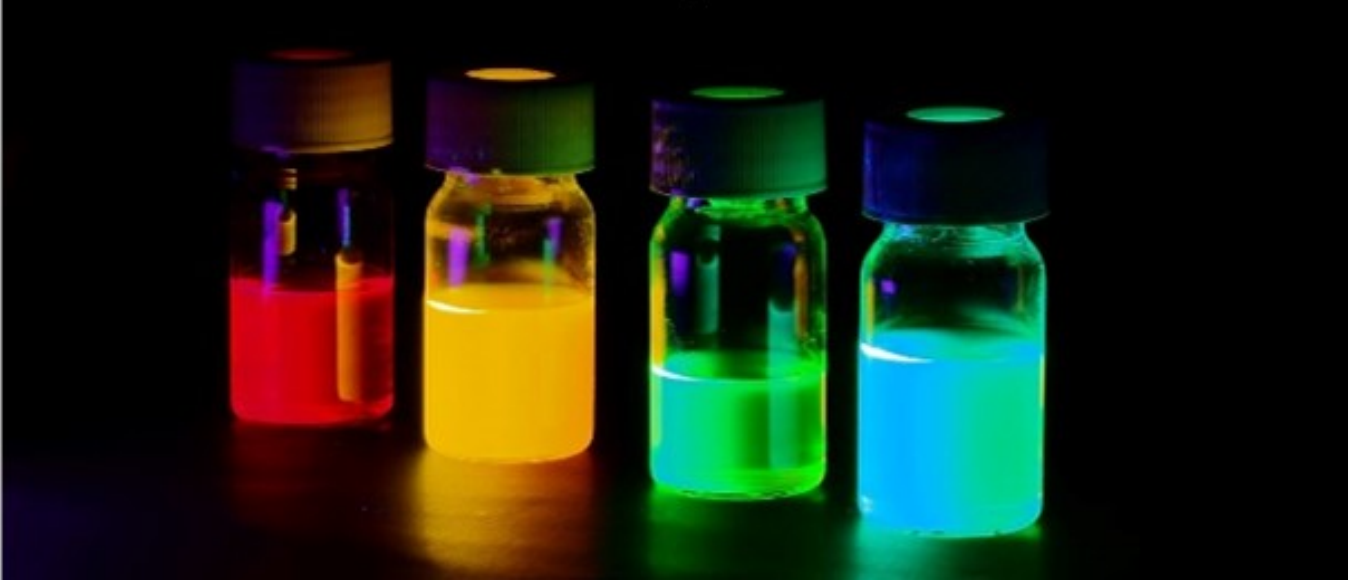

Figure 1.8: A photograph of $\mathrm{CsPbX_{3 }}$ NCs under UV lamp at $365 \mathrm{~nm}$ excitation. The emission was tuned by changing the size and composition of NCs. These perovskite NCs show the emission through the entire visible spectrum (490-680 $n m)$. 
NCs with different layered crystal structures showing $20 \%$ PL quantum yield [90]. Later on, Bhaumik et al. enhanced the PL quantum yield to $95 \%$ in green spectral region by growing an octylammonium shell on the NCs [18]. Minh et al. synthesised colour tuneable formamidinium lead halide perovskite NCs with maximum quantum yield of $75 \%$ [19]. However, organic-inorganic hybrid perovskites were found to have thermal instability towards decomposition and even at moderate temperature, $\left(\approx 60{ }^{\circ} \mathrm{C}\right)$ the material starts decomposing to the corresponding solid phase of lead (II) halide and gaseous phase of methylamine and hydrogen halide [91]. This is not ideal for long term stability in optoelectronic devices. Therefore, a pure inorganic perovskite was needed to overcome this thermal instability. In 2015, Protesescu et al. reported the synthesis of pure inorganic metal halide perovskite $\mathrm{NCs}, \mathrm{CsPbX}_{3}(\mathrm{X}=\mathrm{Cl}, \mathrm{Br}$, I), by hot-injection colloidal route [17]. They reported the maximum PL quantum yield of $90 \%$ with emission tuneability through the entire visible spectrum (419-700 nm) [17].

\subsection{LEDs}

A LED is a semiconductor device which emits light when an electric field is applied. In other words, LED is the semiconductor device which convert electricity into light. LED have the similar characteristics to a pn junction diode, that means the current only flows in the forward bias. A traditional LED is usually made of a very thin layer of semiconductor, which emits colour of certain wavelength when an electric field is applied. A standard LED has long life time with relatively fast responsivity and require very low input voltage of $3 \mathrm{~V}$ and current of $\approx 50 \mathrm{~mA}$, which makes the total output power of $\approx 150 \mathrm{~mW}$. LEDs are very energy efficient and therefore widely used in lighting industry and display technology to reduce energy usage.

There are three main solid state light sources in the current market. Incandes- 
cent bulb, compact fluorescent bulb, and LEDs. Figure 1.9 shows the photograph of these three light sources from Philips, which are being used in our life on daily basis.

The incandescent bulb was the first invented and most popular light source in nineteenth century, which emits a broadband emission when the metal wire is heated by electricity. The efficiency of incandescent bulb is very low as only $5 \%$ of input energy is converted to visible light and remaining $95 \%$ is converted to heat. Additionally, they have relatively short lifetime of 1500-2000 hours [92]. In a compact fluorescent bulb, the electricity is used to excite a low pressure gas which produces a narrow band emission. They have much higher efficiency than those incandescent bulb due to their narrow emission line in the visible spectrum with a relatively long time life time of 10,000-20,000 hours. However, compact fluorescent bulb takes longer time to start-up, use mercury and other toxic materials, and display visual flickering [93]. The colour rendering in compact fluorescent light course is very poor, which makes its application very limited in display technology. The most recent and efficient technology in solid state lighting is LEDs. LEDs use the electricity to excite and recombine the elec-

a)

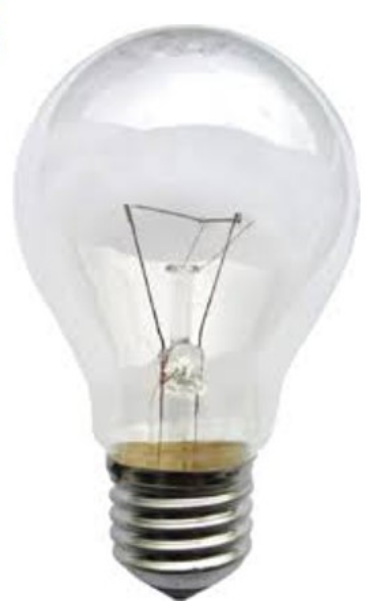

b)

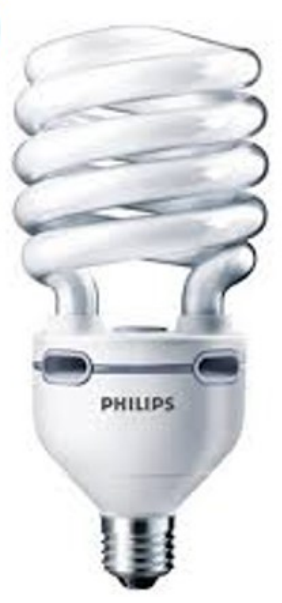

c)

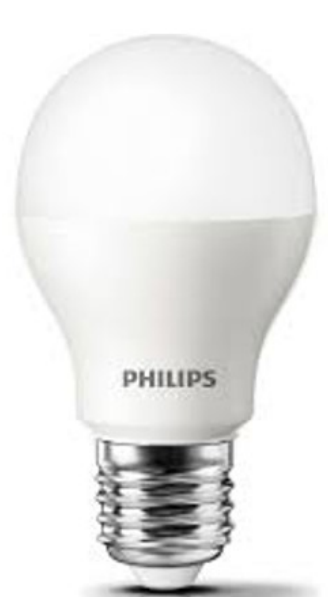

Figure 1.9: A photograph of electricity driven light sources from Philips. a) Incandescent bulb, b) compact fluorescent bulb, and c) LED. 
trons to emit light with very low input energy. Additionally, LEDs have a much longer lifespan of 100,000 h, which is longer than other lighting sources [94].

\subsubsection{Working Principle}

LED works on the principle of electroluminescence (EL), which is the emission of light from a semiconductor in the presence of electric field. Traditional LEDs are made of a semiconducting material, typically AlGaAs. The entirely pure form of this materials can not conduct electricity because there is a perfect bonding between atoms, which leaves no free electron to conduct. However, an impurity can create free electrons and holes to allow it to conduct the electricity. When voltage is applied, the negative charged electrons are forced to move one direction and the positive holes move to the other direction. When a free electron comes near a hole, it combines with the hole. The hole exists at

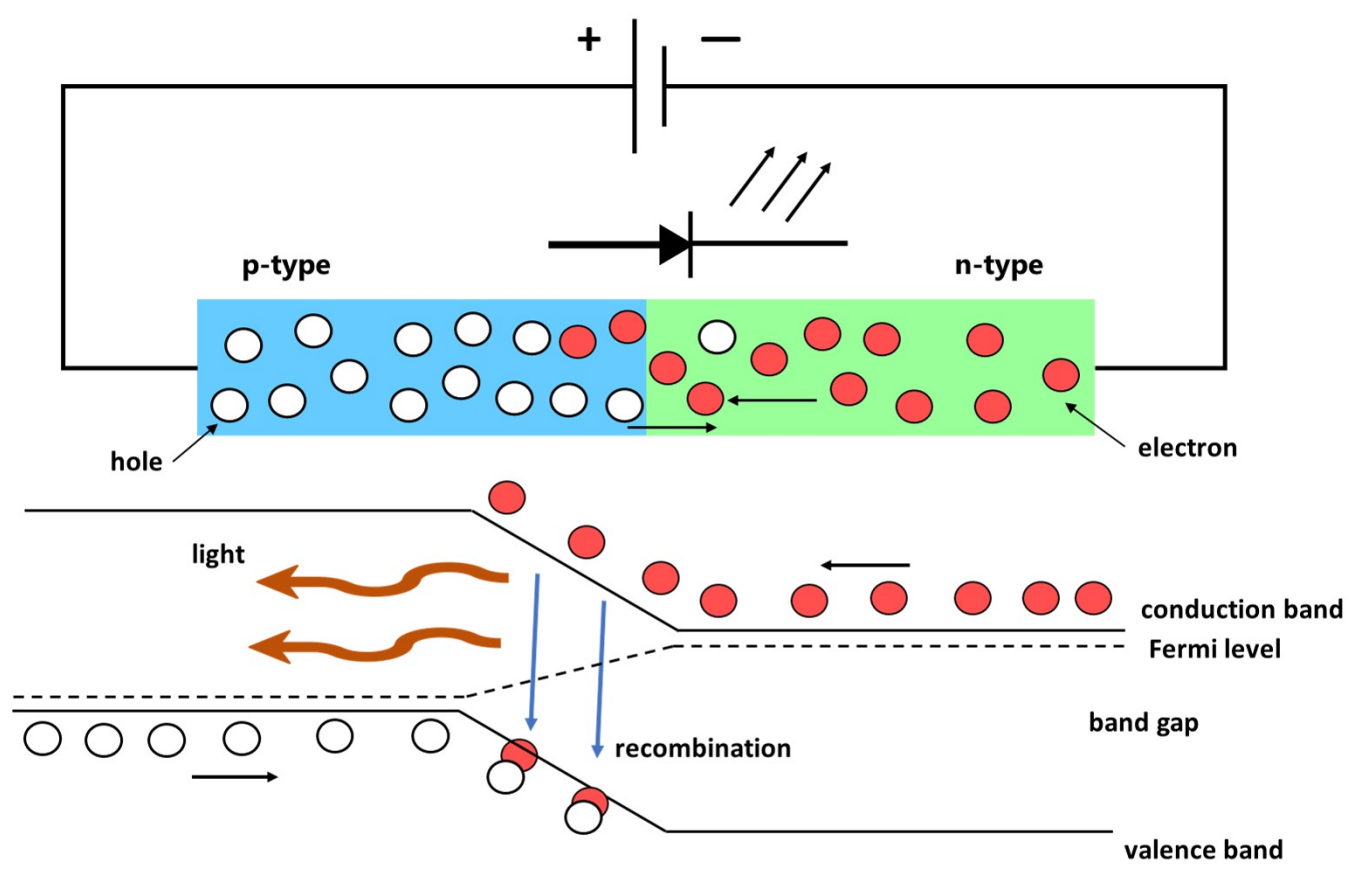

Figure 1.10: The working principle of a traditional LED with band diagram. Emission of the photon occurs when an electron recombines with a hole. 
the lower energy level than the free electron so the electron must lose energy to combine with the hole. This energy is released in the form of a photon. The amount of photon energy released determined the colour or energy of the light.

LEDs contains a doped semiconductor that can either be called $\mathrm{n}$ type material, where the charge carriers are electrons or $p$ type material, where the charge carriers are made up from absence of electrons called holes. An LED is composed of these two type of materials deposited on top of each other together and creating a pn junction. When a forward bias is applied, electrons from the $\mathrm{n}$ region move towards the $\mathrm{p}$ region and recombine with the holes. Electrons exist in the conduction band and holes in the valence band (Figure 1.10). Conduction band has higher energy level than valence band therefore, electrons emit some portion of energy in the form of photon and this energy is equal to the band gap of the material. If a material has higher band gap the emitted light will have a lower wavelength and if the band gap is lower the emitted light will have higher wavelength. It should be noted that, some materials show recombination energy loss in the form of heat, such as silicon or germanium [94].

\subsubsection{History of LEDs}

LED is based on the phenomena called EL, which was first discovered by Captain Henry Joseph Round on a silicon carbide crystal in 1907 [95]. EL from ZnS powder was also observed by Georges Destriau in 1936 [96]. EL in infrared region was also observed in GaAs, GaSb, SiGe, and InP alloys [97]. Several other researchers investigated EL in visible and IR spectral region [96, 98]. In 1966, The first infrared practical LED was invented using GaAs [99]. Later on, Texas Instruments (TI) commercialised a GaAs infrared (IR) LED emitting at $890 \mathrm{~nm}$ (near IR) [98].

The first visible LED, with emission in the red spectral region, using $\mathrm{Ga}\left(\mathrm{As}_{1-x} \mathrm{P}_{x}\right)$, was reported by Nick Holonyak Jr. in 1962 [100]. Later on, M. George Craford 
improved the efficiency of red-orange LEDs up to 10x [101]. In addition, he also reported the first yellow LED [101]. Herbert Paul Maruska was the first one to develop the blue LED using GaN on sapphire substrate in 1972 and awarded a Nobel prize for his invention [102]. Later on, an American company, Cree, invented a blue LED using SiC [103]. However, all of these LEDs displayed poor brightness and low efficiency. Shuji Nakamura invented the first bright blue LED using InGaN in 1992 [104]. Other researchers also developed the efficient blue LEDs using variety of materials, such as gallium nitride, transparent indium tin oxide, GaAs, and InGaN [105-107]

After the successful invention of blue LEDs, researchers turned their attention towards the construction of white emitting LEDs. The first white LED contains a blue emitter coated with $\mathrm{Y}_{3} \mathrm{Al}_{5} \mathrm{O}_{12}$ :Ce known as yttrium aluminium garnet (YAG) phosphor. The YAG coating on the blue emitter absorbed some blue light and emitted yellow light. This yellow light with remaining blue light from the emitter seems white to the human eye [108]. Later on, researchers have used variety of phosphors to produce light in the green and red spectral region as well. The resulting mixture of red, green, and blue (RGB) not only looks superior to human eye but also showed better colour rendering. To date, mixing RGB is the the most efficient way to produce the white light. These LEDs were still efficient than an incandescent bulb with $300 \mathrm{~lm} / \mathrm{W}$ of luminous efficacy, 100,000 hours of lifetime, and continuous reduction in cost [109]. However, these LEDs were very expensive and not proven to be very efficient due to heat loss [110].

Andrë Bernanose was the first scientist to observe the EL in organic materials [111]. The first organic LED was built in 1987 by American chemists Steven Van Slyke and Ching W. Tang [112]. The organic layer was sandwiched between the hole and electron transport layer in this device. The light emission occurs by recombination of electron and hole in the organic layer. The efficiency of OLED was found to be better than phosphor LEDs [112, 113]. They also exhibit low turn-on voltage, lower cost, flexible display, and wide viewer angle with 

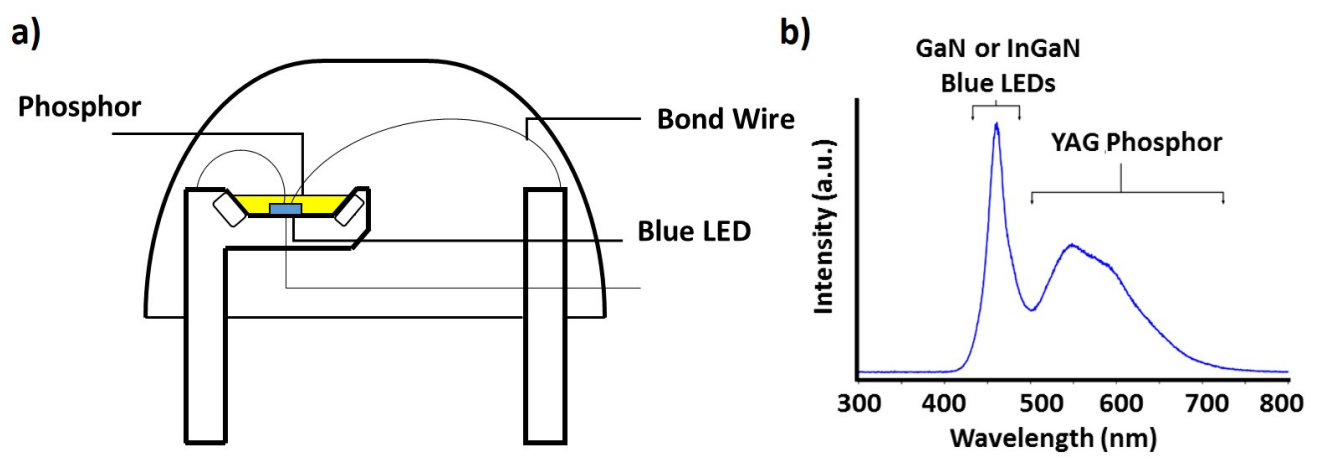

Figure 1.11: a) A schematic of the YAG white light LED and b) its EL spectra [110]

high contrast and brightness $\left(\approx 600 \mathrm{~cd} / \mathrm{m}^{2}\right)$ [112]. Organic molecules conduct electricity due to the delocalisation of pi $(\pi)$ electrons. These materials are organic semiconductors and have highest occupied molecular orbital (HOMO) and lowest unoccupied molecular orbital (LUMO) similar to valence and conduction band in inorganic semiconductors. Figure 1.12 shows the schematic of organic light emitting diode (OLED). When an electric field is applied, electrons transport from cathode to organic layer via electron transport layer, whereas holes transport to the organic layer via hole transport layer. Eventually, electrons from LUMO recombine with hole in HOMO in the organic layer and produce light of a particular wavelength [112]. However, the lifetime of OLED was found to be very limited. The efficiency of commercial OLED was recorded to degrade by $7 \%, 8 \%$, and $12 \%$ in red, green, and blue spectral region after 1000 hours due to the accumulation of non-radiative recombination centers [112]. OLED showed $20 \% \mathrm{EQE}$ at $625 \mathrm{~nm}$ (red spectral region) and $19 \% \mathrm{EQE}$ at 530 $\mathrm{nm}$ (green spectral region) $[114,115]$. However, blue LEDs could only show the maximum EQE of 4-6 \% [116]. In addition, organic molecules are very sensitive to water which limits the practical outdoor application of these devices. Therefore, colour imbalance, shorter life span, high moisture sensitivity have become the major challenges in the application of OLEDs [112].

QDLEDs have similar device structure like OLED except the QD layer is sandwiched between HTL and ETL instead of the organic molecules. Therefore, 

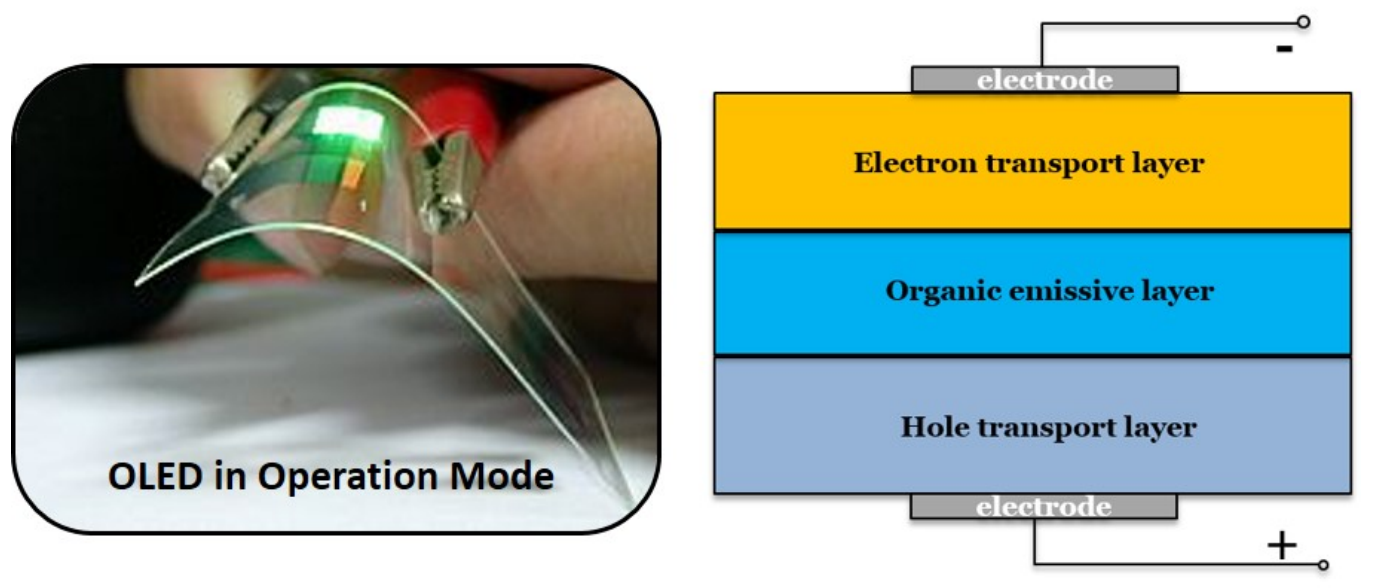

Figure 1.12: (right) A schematic of OLED with (left) a flexible display device in operation mode [113]

electron hole recombination occurs in the QD layer. QDLEDs offer even higher brightness than OLEDs $\left(1500 \mathrm{~cd} / \mathrm{m}^{2}\right)$, longer life spans, cheaper processing costs, low turn-on voltage, better colour rendering, and precise colour tuning with sharper emission and thus combine all the advantages of QDs (Section 1.2) [117]. Figure 1.13b shows a schematic to explain the working principle of

a)

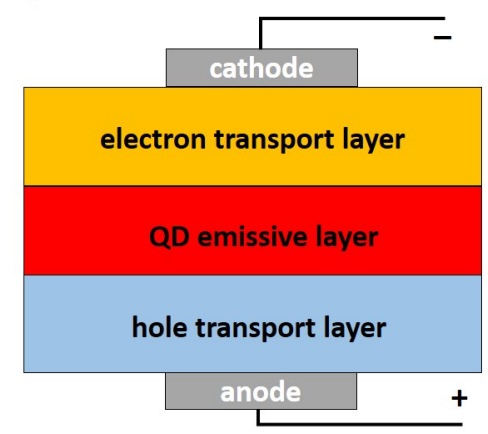

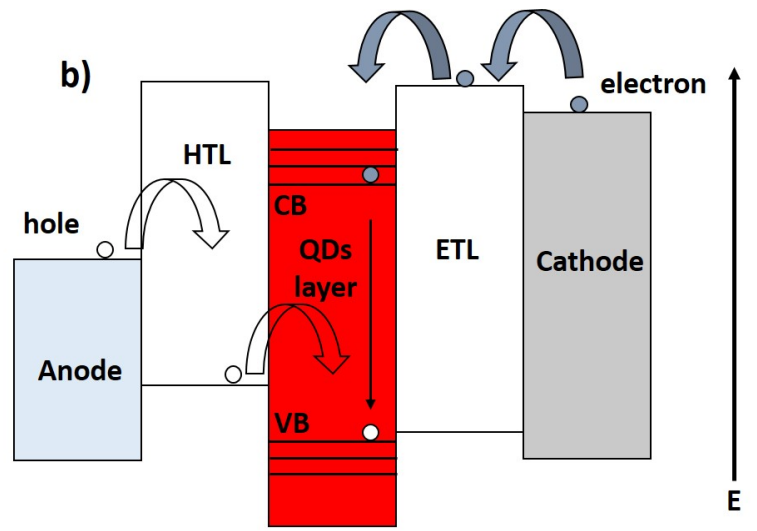

Figure 1.13: a) A schematic of Quantum dot light emitting diode (QDLED), b) a digram showing the working principle of QDLEDs with respect to the energy level. Electrons and holes transports through the ETL and HTL and recombine in QDs layer with an emission of light. 
QD LEDs. It can be seen that the energy level of hole transport layer and electron transport layers are aligned in a way that they transport efficiently towards the QDs layer. Variation in the thickness of HTL and ETL helps to control the rate of electron and hole injection. Eventually, they both recombine in the QD layer and emit a photon with a wavelength depending on the band gap of the QDs [118]. Sometimes, recombination of electron and hole doesn't result in the emission of a photon due to non-radiative recombination, which will be explained in the next section [119]. The most common NCs used in QDLEDs are CdS or CdSe, CuInS 2 , and InP [1, 120-122]. However, to date, CdS/ZnS and CdS/CdSe core-shell QDLEDs have proven to be the most efficient, due to almost $100 \%$ PL quantum yield of these QDs with very stable and tuneable emission spectra in the entire visible region $[1,2,4,11,123]$. This has led to construct CdS/CdSe QDLEDs with a maximum EQE of $20.5 \%$ in 2014 [11]. The only drawback to this technology is that CdSe contains toxic cadmium, a heavy metal banned for use in electronics in some markets [123, 124]. Recently, $\mathrm{CsPbX}_{3}$ perovskite NCs with PL QY as high as $90 \%$ have been reported, suggesting that these could replace CdSe as the active layer in OLED structured devices [17]. Although, $\mathrm{CsPbX}_{3}$ contains a toxic element $\mathrm{Pb}$ in it, but the toxicity of $\mathrm{Pb}$ is less than $\mathrm{Cd}$ and the amount of $\mathrm{Pb}$ in $\mathrm{CsPbX}_{3}$ is less than the amount of Cd in Cd-based LEDs [125]. In perovskite structure, It is also possible to replace $\mathrm{Pb}$ with other metal such as tin or bismuth for the optoelectronic applications $[22,126,127]$. Therefore, researchers began working in the area of perovskite NC LEDs (peNC-LEDs) [20, 30, 128-130]. Over time, significant development has occurred regarding Perovskite LEDs: starting from the perovskite QDLEDs to nanostructured 2D perovskite LEDs and finally, the RuddlesdenPopper phase perovskite LEDs (Section 1.4.3) [28-30, 86] 


\subsubsection{Perovskite in LEDs}

Halides perovskite has been proven to be a promising material not only for photovoltaics but also for LEDs and lasers [16, 131-134] Li et al. prepared a blend of $\mathrm{CH}_{3} \mathrm{NH}_{3} \mathrm{PbBr}_{3} \mathrm{NCs}$ with polyimide precursor (PIP) and incorporated in LED device structure by using an organic electron and hole transport layer [135]. They reported 1.2\% EQE and opened the area in the field of flexible and efficient LEDs [135]. Tan et al. fabricated a near infrared range perovskite based LED $(773 \mathrm{~nm})$ by using a $15 \mathrm{~nm}$ layer of $\mathrm{CH}_{3} \mathrm{NH}_{3} \mathrm{PbI}_{3-x} \mathrm{Cl}_{x}$ perovskite emitter and achieved an EQE of $0.1 \%$ [17]. However, hybrid organic-inorganic metal halide perovskites have significantly less stability and potential than pure inorganic perovskites for optoelectronic applications [136]. For the first time in 2015, Song et al. reported $\mathrm{CsPbX}_{3}$ NCs LEDs with maximum EQE $0.12 \%$ in $\mathrm{CsPBr}_{3}$ devices [21]. They used $\mathrm{PVK}$ as a hole transporting as well as electron blocking layer and TPBi as an electron transporting layer [21]. Zhang et $a l$. has also reported $\mathrm{CsPbBr}_{3}$ NCs LED using poly-TPD as hole transport layer and TPBi as an electron transport layer [130]. They claimed to increase the peak brightness maxima and hole injection efficiency by introducing a perfluorinated ionomer layer between poly-TPD and QDs [130]. Later on, other researchers improved the EQE of perovskite based LEDs [128]. Recently, quasi2D perovskite based LEDs have also gained attention due to their extra ordinary performance [28, 29, 137]. A quasi-2D or Ruddlesden-Popper (RP) phase perovskite is a layered perovskite structure with two dimensional perovskite layers separated with each other by organic cations and form the thicker nanosheets [137]. The thicker material weakens the binding energy and thus combines the colour purity and tuneability of QDs with the conduction properties of 3D crystals [35-37]

The fabrication of QDLEDs is generally conducted using the spin coating technique followed by thermal evaporation of top electrode $[1,20]$. In the spin coating technique, QDs or any material dissolved in a low boiling point solvent. Then, the QDs solution is deposited on the substrate to cover the entire area 
followed by immediate rotation of substrate at high RPM (>1000) for 30-60 sec. Most of the solution sheer away due to the centrifugal force, which results in an even thin film of NCs. Leftover solvent quickly dries due to the spin action. The thickness of thin film depends on the concentration of solution and the RPM $[1,20,138]$. HTL and ETL are also deposited using the spin coating techniques [20]. However, the layer on top of QDs is preferred to deposit by non-solution processed technique, usually thermal evaporation, in order to protect the QDs surface from solvents [20]. Thermal evaporation is a physical technique to deposit a thin film on a substrate, in which a material is heated in a high vacuum to its evaporation point, which is then coated on the substrate to form a thin film [139]

\subsubsection{Carrier Recombination in Semiconductors}

During the recombination process, electrons from the conduction band and holes from the valence band recombine with each other in the emissive layer (Figure 1.14). The amount of energy released depends on the energy of initial and final state of electron. This energy can be released by three different process. These three methods of carrier recombination describe the recombination process [140]

\subsubsection{Radiative Recombination}

The first process is radiative recombination, in which the recombination of an electron and a hole releases the energy in the form of photon. An electron from the conduction band falls into empty valence band, where it recombines radiatively and emits a photon. This process usually occurs in a direct band gap semiconductor and also called as band to band transition [140]. 


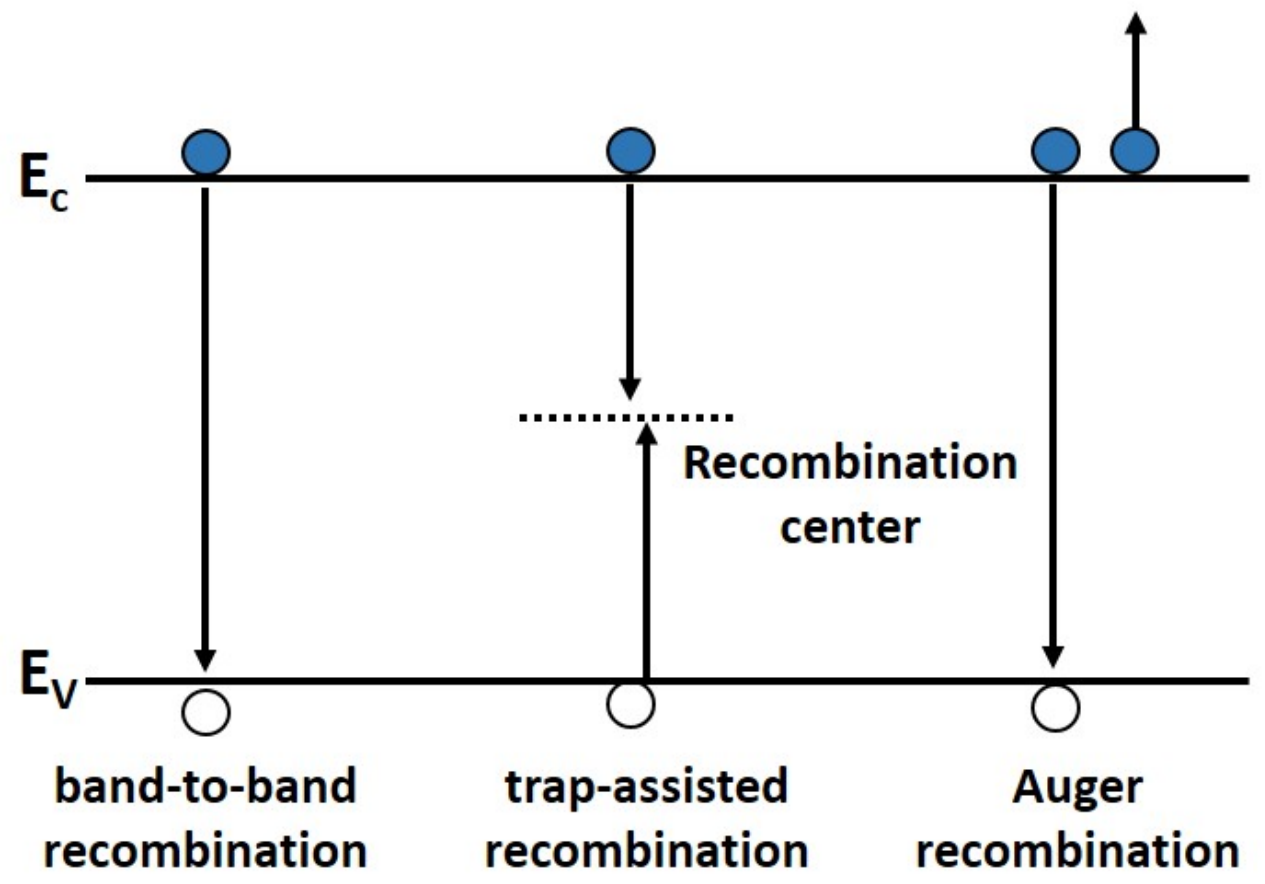

Figure 1.14: An illustration of carrier recombination process in semiconductors. An electron and a hole recombination can occur by three different mechanisms, which results in either radiative or nonradiative recombination.

\subsubsection{Trap-Assisted Recombination}

The second process is called Shockley-Read-Hall (SRH) or trap-assisted recombination, in which the electron from the conduction band falls into an energy level within the band gap called deep-level traps. These deep-level traps are created due to the presence of impurity or foreign atom in the crystals. After a while, the electron from the deep-level trap can fall into an empty valence state to complete the electron-hole recombination process. The trap-assisted process is basically a two step nonradiative recombination process of electron and hole. This process occurs usually in indirect band gap semiconductors. However, it can also occur if the carrier density is very low in a direct band gap semiconductor $[140,141]$ 


\subsubsection{Auger Recombination}

Auger recombination is a non radiative recombination process, where the electron gives its energy to the another carrier before recombination, which is in the higher energy level without moving to a new energy band. After taking the energy, this carrier releases the energy in the form of thermal vibrations. This process only occur if the carrier density is very high [141]. Auger recombination is one of the major reasons for efficiency drop in LEDs [3, 142-144].

\subsection{Solar Cells}

In addition to making LEDs, parts of this work on these materials is to build solar cells. Appendix includes the solar cell side of the project conducted in collaboration with Dr Yingzhuang Ma at Victoria University of Wellington. Some of the perovskite NC samples were also provided to Dr Hannah Zheng for the fabrication of perovskite NCs solar cells.

Solar cell is a semiconductor device which converts the solar radiation into electricity. When the light hits on the surface of the device, it absorbs the light and generate electron hole pairs. At this point, these charge carriers get separated and travel towards the electrode for current to flow.

\subsubsection{Carrier Generation in Solar Cells}

In semiconductors, carriers can be generated with the illumination of light. When a photon interacts with an electron in semiconductor, it excites the electron from lower energy level to a higher energy level. If the photon energy is larger than the band gap of the semiconductor, the electron is excited from the valence band to the conduction band. Thus, the generation of electron-hole 
pair occurs (Figure 1.15). Multiple electron-hole pairs can also be formed if the energy of a photon is much larger than the band gap of the semiconductor in QD based solar cells, which is called multiple exciton generation.

\subsubsection{J-V Characteristic of a Solar Cell}

Figure $1.15 \mathrm{~b}$ shows the $\mathrm{J}-\mathrm{V}$ curve of a solar cell fabricated in our research group. When the cell was illuminated with the light of calibrated solar lamp, the current shifts in the $4^{\text {th }}$ quadrant. The J-V curve helps to calculate the power conversion efficiency of a solar cell. The term $\mathrm{V}_{o c}$, labelled on the graph, stands for open circuit voltage which means the voltage at zero current. $\mathrm{I}_{s c}$ stands for short circuit current which means the current at zero voltage. $\mathrm{M}_{p p}$ is the maximum power point, where the supplied power is at maximum value. $\mathrm{V}_{m p}$ is the maximum voltage power point and $\mathrm{I}_{m p}$ is the maximum current power point.

$$
M_{p p}=V_{m p} \times J_{m p}
$$

The fill factor (FF) of a solar cell defines the squareness of J-V curve in $4^{\text {th }}$ quadrant. it is the ratio between the maximum power point $\left(\mathrm{M}_{p p}\right)$ and the product of $\mathrm{V}_{o c}$ and $\mathrm{J}_{s c}$.
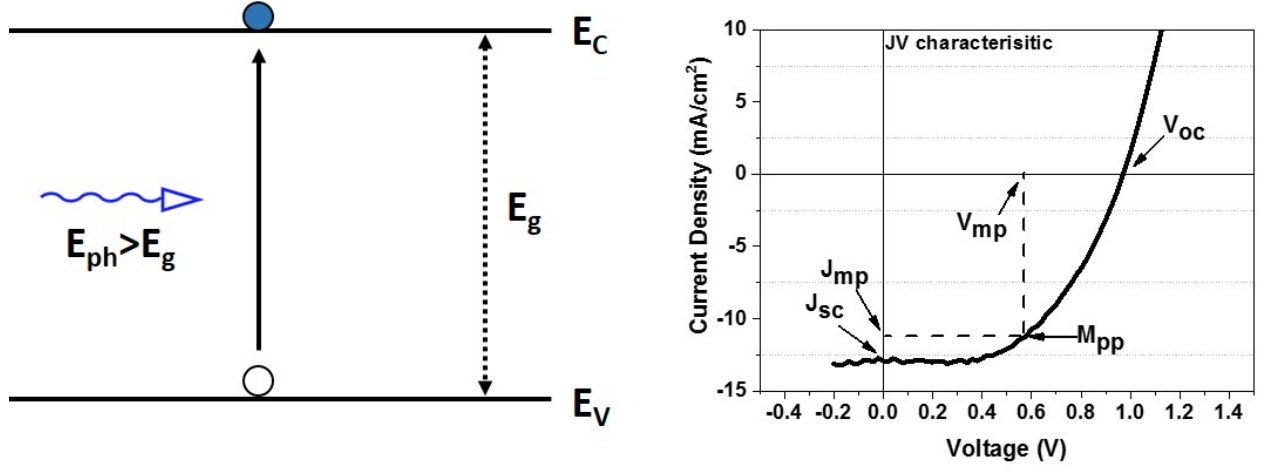

Figure 1.15: a) An illustration of carrier generation in solar cells and b) current density vs. voltage characteristic of a solar cell fabricated in the Halpert Group. 


$$
F F=\frac{V_{m p} \times J_{m p}}{V_{o c} \times J_{s c}}
$$

The output power of a solar cell is the multiplication of $\mathrm{V}_{o c}, \mathrm{~J}_{s c}$, and FF.

$$
P_{\text {output }}=V_{o c} \times J_{s c} \times F F
$$

Power conversion efficiency $(\eta$ ) of a solar cell is the ratio between input power and the output power.

$$
\eta=\frac{P_{\text {output }}}{P_{\text {input }}}
$$

The input power of the solar cell was the power of the solar lamp $\left(100 \mathrm{~mW} / \mathrm{cm}^{2}\right)$ and the output power of a solar cell is calculated using Equation 1.35.

$$
\eta=\frac{V_{o c} \times J_{s c} \times F F}{P_{\text {input }}}
$$

\subsubsection{Perovskites in Solar Cells}

Perovskite has shown excellent properties for solar cell applications. In less a decade the power conversion efficiency of perovskite solar cells has reached very close to the commercially available Si solar cells. The National Renewable Energy Laboratory (NREL) for solar cell efficiency also suggested that the perovskite device has the highest rate of efficiency increase than other types of devices (Figure 1.16). However, the stability is yet to be resolved in perovskite photovoltaics.

The first reported perovskite solar cells were made in 2006 using $\mathrm{CH}_{3} \mathrm{NH}_{3} \mathrm{PbBr}_{3}$ and $\mathrm{CH}_{3} \mathrm{NH}_{3} \mathrm{PbI}_{3}$ as a sensitizer in a dye-sensitized solar cell (DSSC) and achieved $0.36 \%$ and $2.19 \%$ power conversion efficiency (PCE) respectively [145]. In 2009, Kojima et al. reported a similar type of DSSC and achieved 3.8 \% PCE for $\mathrm{CH}_{3} \mathrm{NH}_{3} \mathrm{PbI}_{3}$ and $3.1 \%$ efficiency for $\mathrm{CH}_{3} \mathrm{NH}_{3} \mathrm{PbBr}_{3}$ [146]. Initially, these devices were reported to have only 3-4 \% PCE. Later, Park's group at Sungkyunkwan 
University (S. Korea) used a spin coating technique to deposit methylammonium lead iodide $\left(\mathrm{MAPbI}_{3}\right)$ perovskite sensitizers on a thin film of $\mathrm{TiO}_{2} \mathrm{NCs}$ surface and achieved $6.5 \%$ PCE. These materials were found to be more stable in electrolyte solutions [147]. Due to the dissolution problem of OMH perovskite devices in polar liquid electrolyte, perovskite devices were not found to be successful as DSSCs and this led researchers to focus on solid-state perovskite devices. Kim et al. used a solid state semiconductor organic molecule (spiroMeOTAD) and perovskite instead of the liquid based hole transport layer into titanium dioxide scaffold and they achieved $9.7 \%$ efficiency [148]. Later Lee $e t$ al. replaced $\mathrm{TiO}_{2}$ by $\mathrm{Al}_{2} \mathrm{O}_{3}$ and obtained an increment in efficiency to $10.9 \%$ [149]. Liu et al. also fabricated perovskite devices using a planar hetrojunction, planar interface between layers of two different crystalline semiconductor materials, and achieved $15.4 \%$ PCE [150]. Jeon et al. used the combination of planar and scaffold layer of bromide-iodide mixed halide (methylammonium lead bromide-iodide) with optimised thickness of both layers and chemical compo-

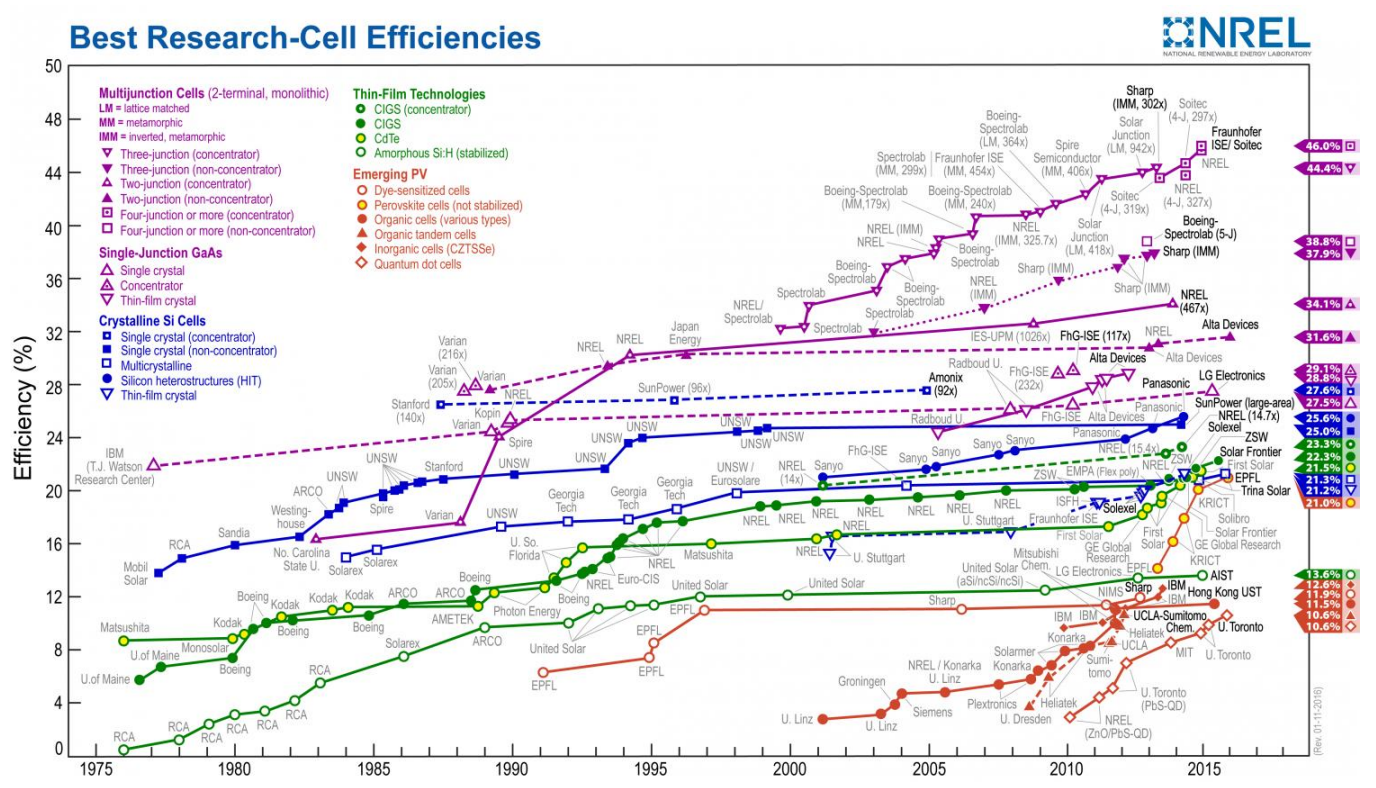

Figure 1.16: A graph showing the increase in efficiency over time for highly efficient solar cells. Credit: NREL. 
sition, eventually they were able to achieve $16.2 \%$ of PCE with no hysteresis [151]. Noh et al. have been working to improve device efficiency by tuning the chemical composition of the material in order to absorb light of the entire solar spectrum [152]. In 2017, Yang et al. further improved the PCE to $22.1 \%$ by addition of triiodide ion during the formation formamidinium lead iodide films, which resulted in the reduction of deep level defects in the perovskite crystals [153]

\subsection{Methods}

The synthesis of NCs and device fabrication are explained in each chapter in detail. This section explains the sample preparation and basic principles of characterisation techniques used in this work. Nanoparticles were characterised by microscopy including transmission electron microscopy (TEM) and scanning electron microscopy (SEM) for structural analysis. X-ray diffraction (XRD), ultraviolet-visible (UV-Vis) absorption spectroscopy, photoluminescence (PL) spectroscopy, and transient absorbance spectroscopy were used, in collaboration with the Hodgkiss group, to analyse and monitor the detailed properties of nanoparticles. The device fabrication process involves the spin coating and the thermal evaporation technique followed by measurements of its electrical properties.

\subsubsection{TEM}

TEM is a microscopy technique to analyse the shape, size and morphology of NCs. In this technique, an electron beam is transmitted through an ultrathin layer of specimen. The magnification of TEM is up to about 1,000,000x. An ultra-high vacuum, high voltage, and condenser, objective, and projector lenses are important parts of a TEM. The electron beam which is emitted by a 
filament is focused and concentrated by condenser lenses onto the sample. The transmitted beam consists of elastically scattered electrons which pass through the objective lenses and form the image of the illuminated portion of the specimen that is magnified and enlarged further by the intermediate and projector lens. The formed image is shown on a fluorescent screen and detected by a CCD camera. A Jeol TEM 2100 was used for the optical analysis in this project. All of the samples were dispersed in a suitable solvent followed by sonication for two min. The NCs solution was then drop casted on a Cu-200 mesh on formvar grid followed by plasma cleaning for $10 \mathrm{~min}$.

\subsubsection{SEM}

A scanning electron microscope uses an electron beam to produce an image of a sample by scanning it. The electron beam is generated by a tungsten filament in the electron gun, which is then passed through electromagnetic lenses and apertures to produce a focused beam. The interaction of the beam with the sample causes electrons to be removed from the sample. These are known as secondary electrons. These electrons are amplified and sent to a detector. The electron beam scans everywhere on the sample to cover the entire area. The whole process occurs under the vacuum and the sample must be conductive to analyse via SEM. Otherwise, a thin layer of conductive coating can be applied on the sample by sputter coating technique. The Jeol 6500 was used to analyse the thin films.

\subsubsection{XRD}

XRD helps to study the crystal structure of samples. Additionally, it can also provide the estimation of crystalline size using Scherrer equation [154]. XRD is a non-destructive, versatile method to analyse the chemical composition and crystallographic information of a material. When a material is bombarded with 


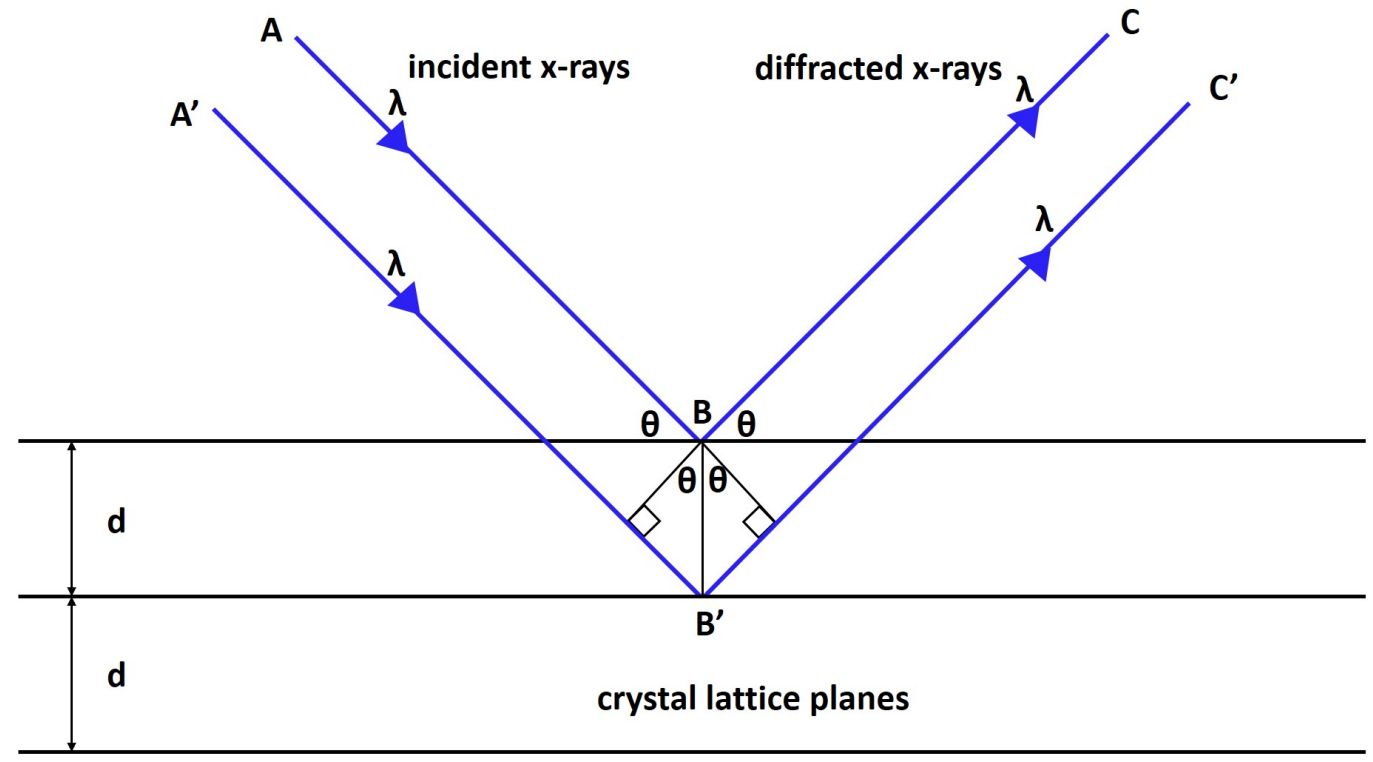

Figure 1.17: An illustration on the basic principle of XRD spectroscopy using Bragg's diffraction law.

a continuous beam of X-rays, they are scattered in all directions. In some directions, the diffracted radiation is intense due to constructive inference between planes. The diffracted pattern is recorded by a goniometer. Constructive interference occurs, if the path difference between the rays is an integral multiple of wavelength, which is Bragg's diffraction law [154]

$$
\lambda=2 d \sin \theta
$$

Scherrer equation was used to estimate the particle size for some of the samples. This equation can help to relate the decrease in particle size with the width of diffraction peaks [154]. The decrease in the particle size results in the broadening of diffraction peaks. This effect has been observed in nanocrystalline materials $[8,20]$

$$
\tau=\frac{K \lambda}{\beta \cos \theta}
$$


Where, $\tau$ is the crystalline size, $\mathrm{K}$ is the shape factor, which depends on shape of the crystal, $\lambda$ is the wavelength of X-rays, $\beta$ is the full width at half maximum (FWHM) of diffraction peak, and $\theta$ is the Bragg angle. NCs samples, for XRD, were prepared by drop casting the concentrated NC solution (in hexane) on to a glass slide. The XRD patterns were recorded by a PANalytical X-Ray diffractometer using $\mathrm{Cu}-\mathrm{K} \alpha$ radiation, with an operating voltage of $45 \mathrm{kV}$ and a current of $40 \mathrm{~mA}$.

\subsubsection{UV-Visible Spectroscopy}

Absorption spectroscopy provides the details of materials optical properties such as band gap. The UV-Vis spectroscopy is the absorption or transmittance spectroscopy in the UV and visible region. This technique involves the interaction of UV-visible range electromagnetic radiation with a sample. Non-bonding electron (n-electron) or $\pi$-electron contained molecules absorb the UV-Vis range energy and therefore excite the electrons from the ground state [155]. UV-Vis is based on the Lambert Beer's law. This relationship shows the linear dependence between absorbance and concentration of the absorbing species [156, 157].

$$
A=\log \frac{I_{o}}{I}=\epsilon \times c \times L
$$

Where $\mathrm{A}$ is the absorbance, $\mathrm{L}$ is the path length of the sample, $\mathrm{c}$ is the concentration of sample, $\mathrm{I}_{o}$ is the intensity of the incident light, and I is the intensity of transmitted light through the sample. $\varepsilon$ is the extinction coefficient or absorptivity, which defines how strongly a material can absorb light per unit molar concentration at a given wavelength. In this work, a Cary $50 \mathrm{UV}$-Visible spectrophotometer was used. NCs were measured in the form of solution and a $10 \mathrm{~mm}$ quartz cuvette with only solvent was used to create the baseline. For the device and thin film measurements, the sample was directly placed in the spectrometer and a blank film was used to create the baseline. 


\subsubsection{PL Spectroscopy}

A PL spectroscopy provides the details about the emission properties of materials. Additionally, it can provide an estimation on the monodispersity in the sample by FWHM of the emission peak. As NCs synthesised in this work were used for optoelectronic applications, therefore PL spectroscopy is significantly important. PL is the emission from a species after absorption of some photons. PL spectroscopy is basically a nondestructive, contactless method to investigate the electronic structure of any species. In this method, light of a given wavelength photo excites the sample. After some time, the electron returns to the ground level and emits some light in the case of radiative process (1.4.4.1) [157].

Figure 1.18 shows the schematic representation of a PL spectrometer. The system contains two monochromators. In order to measure the PL, the excitation wavelength is chosen by excitation monochromator and the emission is

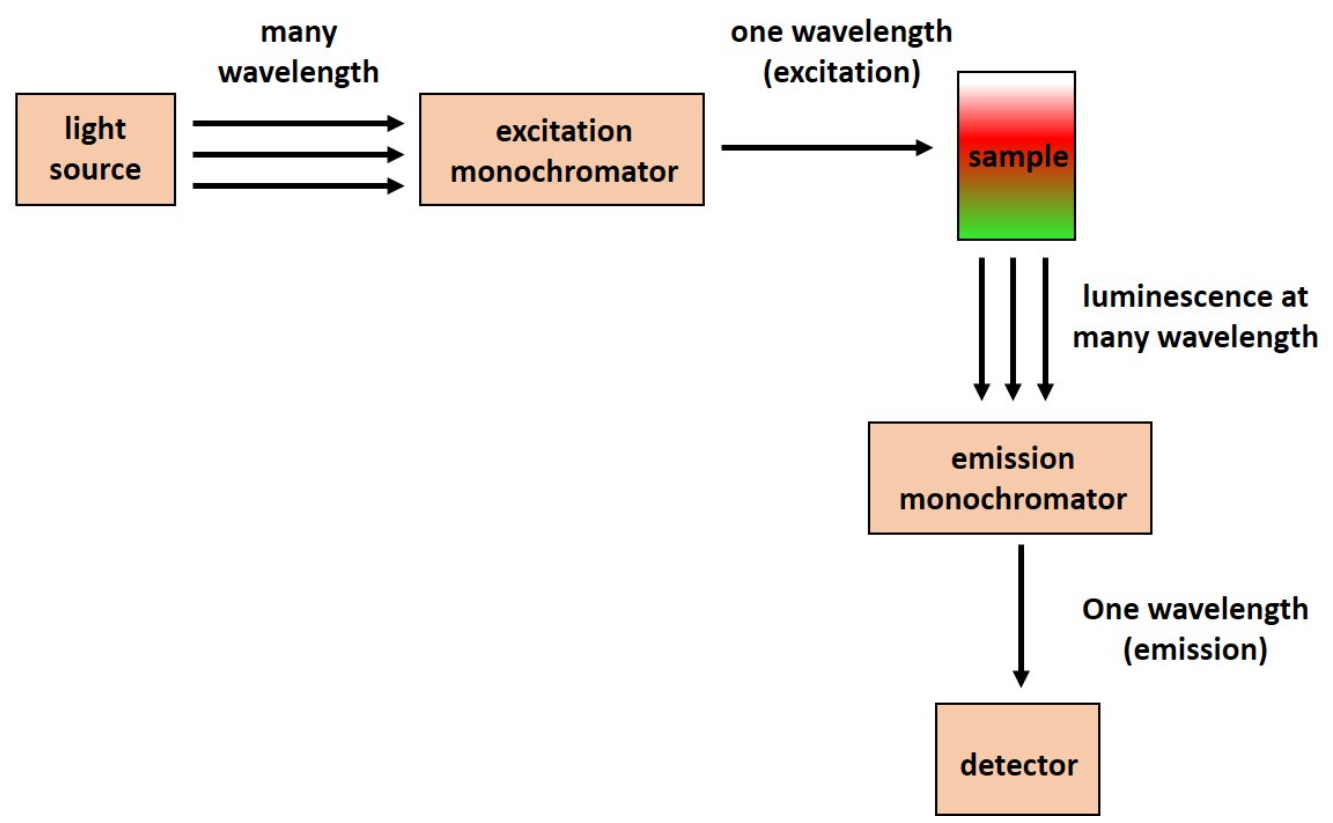

Figure 1.18: A schematic diagram of PL spectrophotometer. 
scanned by the emission monochromator. Both monochromators are usually perpendicular to each other in order to mitigate the scattering of emitted light [157].

A Horiba Fluorolog-3 Spectrometer was used for the measurements. The baseline for the blank was created using a $10 \mathrm{~mm}$ quartz cuvette with pure hexane for the samples in solution form. In the case of NC thin film, a blank substrate with the underneath layers was used to create a baseline.

\subsubsection{J-V Characteristic}

$\mathrm{J}-\mathrm{V}$ measurement provide details about the electrical properties of LEDs, such as turn-on voltage, break down voltage, resistance, and current. In order to emit any radiation, the device requires a current flow. The LED was connected to the power supply and voltage vs current was recorded with EL spectrum. In our case, the Keithley 236 source measurement unit was used for the I-V measurement. The current was converted to current density in order to plot the J-V curve. The current density was calculated using the formula below:

$$
J=\frac{I}{A}
$$

Where, $\mathrm{J}$ is current density, $\mathrm{I}$ is current and $\mathrm{A}$ is the device area, which is 0.06 $\mathrm{cm}^{2}$. Figure 1.19 shows the standard J-V curve of the perovskite LEDs, which has the same characteristics as a pn junction diode. There is a sudden drop in the current which shows the device was not functional after $\approx 8.1 \mathrm{~V}$. The turnon voltage for LED can also be found by J-V curve and is labelled on the graph. 
a)

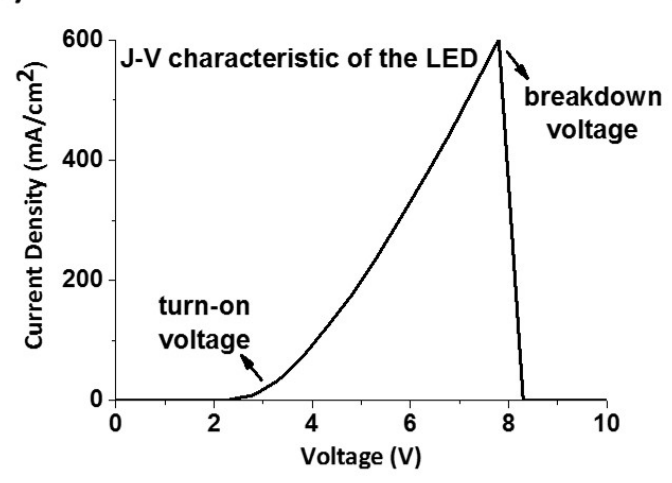

b)

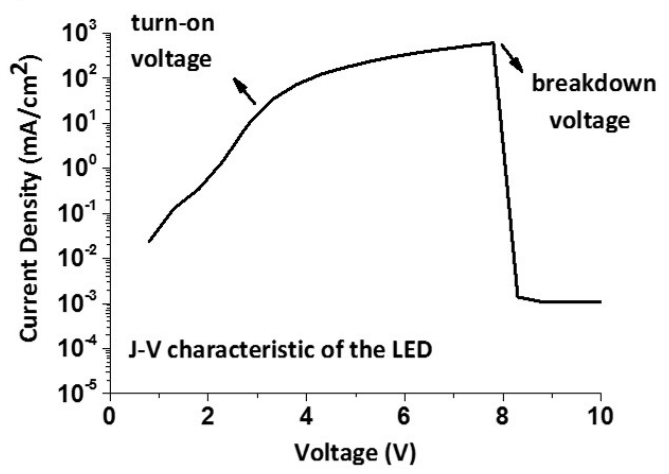

Figure 1.19: A J-V curve of perovskite LEDs a) on a linear scale and b) on a semilog scale.

\subsubsection{EL Measurement}

In this phenomena, the material emits light when the electric field is applied. Therefore, the fabricated LED was connected with a positive and a negative terminal of corresponding electrode. The positive side of the electrode was connected to ITO and negative side of the electrode was connected to the $\mathrm{Al} \mathrm{elec-}$ trode. The voltage was slowly increased up to 3.0-5.0 $\mathrm{V}$ depending on the turning on voltage of LEDs and then IV curve was recorded throughout the measurement.

The EL measurements were carried out by an Ocean Optics integrating sphere $500 \mathrm{~mm}$, ISP-50-8-1, connected to an ocean optics QEPro spectrometer using a $80 \mu \mathrm{m}$ optical fiber or using a QE pro ocean optics spectrometer connected with a $200 \mu \mathrm{m}$ optical fiber (QP200-2VIS-NIR). The fiber was carefully calibrated with an ocean optics HL-3 plus VIS-NIR light source. A Lambertian profile was observed and assumed in the EQE calculation [34, 158]. EL measurements along with the JV curve provide the details on EQE, luminance $\left(\mathrm{cd} / \mathrm{m}^{2}\right)$, luminous efficacy $(\mathrm{lm} / \mathrm{W})$, current efficiency (cd/A) and Commission Internationale de l'Eclairage (CIE) coordinates.

The amount of light power per unit steradian of solid angle in a certain direction 
a)

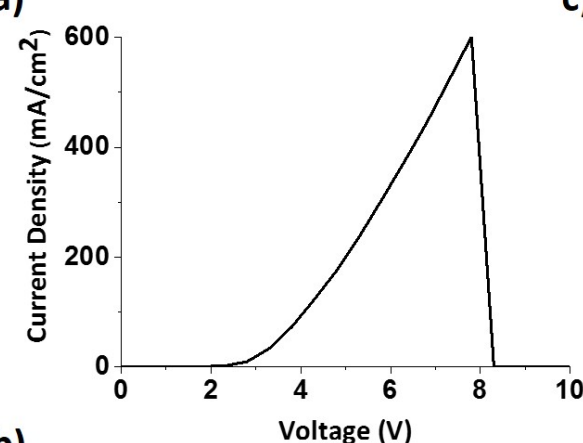

b)

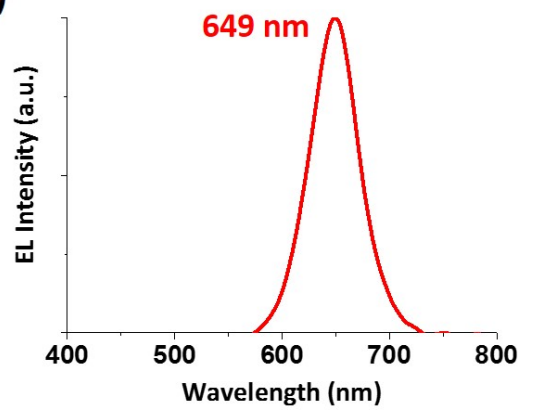

c)

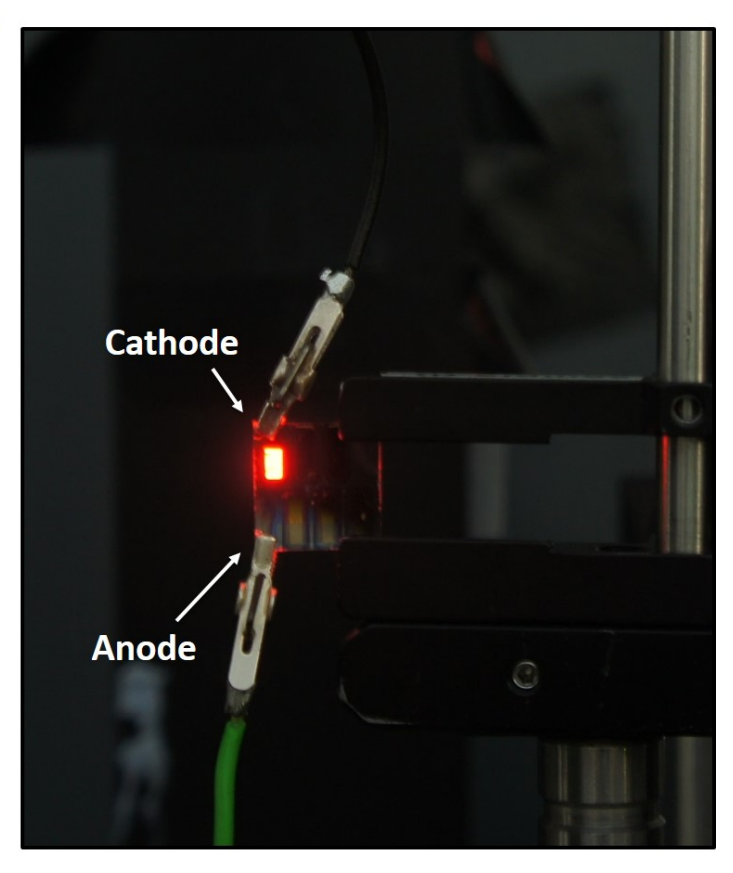

Figure 1.20: a) A JV curve of perovskite LEDs b) EL spectrum of a perovskite LEDs emitting a $649 \mathrm{~nm}$, and c) A photograph of the same perovskite LED emitting at $649 \mathrm{~nm}$. Anode (ITO) was connected with a positive and cathode was connected with a negative (Al) terminal.

emitted by a light source is called luminous intensity, which has candela (cd) unit. Thus, luminous intensity per unit area of light traveled is called luminance $\left(\mathrm{cd} / \mathrm{m}^{2}\right)$. Similarly, luminous intensity per unit current flow is current efficiency (cd/A). Luminous flux is the total amount of total light emitted by a light source, which has lumens $(\mathrm{lm})$ unit. Therefore, luminous efficacy is the ratio between luminous flux and input power (watt).

In this work, the EL spectra were recorded at a different voltages and time intervals. The Ocean Optics spectrometer provides the data in wavelength vs $\mu \mathrm{W} / \mathrm{nm}$ for each EL spectrum, Which was converted to $\mu \mathrm{W} / \mathrm{nm} / \mathrm{sr}$, after dividing by $\pi$. Then, luminous intensity (cd) was calculated using the following formula: 


$$
\text { luminous intensity }(\mathrm{cd})=683 \times l m_{c} \times(\mu W / \mathrm{nm} / \mathrm{sr})
$$

Where, $\operatorname{lm}_{c}$ is the luminous efficacy coefficient, which is a function of the sensitivity of the human eye at different wavelengths [159]. Maximum luminous efficacy is $683 \mathrm{~lm} / \mathrm{w}$ for the human vision [159]. The current efficiency (cd/A) was calculated by taking the ratio of luminous efficacy (Equation 1.42) and the current flowing in the device. Thus, the current efficiency will be:

$$
\text { current efficiency }=\frac{\text { luminous efficacy }(\mathrm{cd})}{\text { current in the device }(\mathrm{A})}
$$

Similarly, the luminous efficacy ( $\mathrm{lm} / \mathrm{W})$ was calculated by using the following formula:

$$
\text { luminous efficacy }(\mathrm{lm} / \mathrm{w})=\frac{\text { luminous efficacy }(\mathrm{cd}) \times \pi}{\text { current }(\mathrm{A}) \times \operatorname{applied} \text { voltage }(\mathrm{V})}
$$

Thus, the EQE is the ratio between the number of photons emitted from the device $\left(\mathrm{N}_{p}\right)$ and the electrons $\left(\mathrm{N}_{e}\right)$ injected into the device.

$$
N_{e}=\text { Current } \times 1.6 \times 10^{-19}(\text { charge of electron })
$$

Energy per photon can be calculated using the following formula:

$$
E_{p}=\frac{h c}{\lambda}
$$

Where, $\mathrm{h}$ is the Planck constant (6.626176 $\times 10^{-34}$ joule-seconds), $\mathrm{c}$ is the speed of light $\left(3.0 \times 10^{8} \mathrm{~m} / \mathrm{s}\right)$ and $\lambda$ is the wavelength which was taken from the EL spectrum. The number of photons emitted from the device can be calculated using the following formula:

$$
N_{p}=(W / n m) E_{p}
$$


Where, $\mathrm{W} / \mathrm{nm}$ was extracted from the EL spectrum and $\mathrm{E}_{p}$ was calculated in Equation 1.46. Thus, the EQE is the ratio between Equation 1.47 and 1.45, which is the number of photons emitted to the electrons injected into the device.

$$
E Q E=\frac{N_{p}}{N_{e}}
$$

For each spectrum, the EQE was calculated at each wavelength and the average of EQE was considered to be the net EQE of that spectrum. For each pixel, several spectra were recorded at different voltages and times, EQE, $\mathrm{lm} / \mathrm{W}, \mathrm{cd} / \mathrm{m}^{2}$, $\mathrm{cd} / \mathrm{A}$ for all of the spectra were calculated and plotted against voltage, current density, and time to see the rate of change in each device. All of these analysis are included in Chapters 3 and 4.

The CIE coordinates, derived from 1931 colour space model, were also calculated for each LED. CIE defines the quantitative relation between physiological perceived colours in human colour vision and physical pure colour in the electromagnetic visible spectrum (Figure 1.21b). CIE 1931 RGB colour space or XYZ colour space were invented by the International Commission on Illumination (CIE) [160]. Cone cells in the human eye have sensitivity at three different spectral wavelengths; 420-440 $\mathrm{nm}$ in short ( $\mathrm{S}$ cone) , 530-540 $\mathrm{nm}$ in middle (M cone), and 560-580 $\mathrm{nm}$ in long (L cone) wavelengths (Figure 1.21a). The human eye is more sensitive towards the green spectral region than it is for the blue and the red spectral regions. That means the brightness of green light appears to be higher than blue and red light. Therefore, the perceived brightness of different wavelengths is the function of $\mathrm{M}$ cone (530-540 nm). The CIE model defines $\mathrm{Y}$ as luminance, $\mathrm{Z}$ as short cone response, and $\mathrm{X}$ as a linear combination of cone response curves [161]. Thus, $\mathrm{Y}$ and $\mathrm{Z}$ describe the chromaticity while $\mathrm{X}$ describes the luminosity related to the eye's detection.

$$
X=\lambda \times \bar{x} \times \delta \lambda
$$


a)

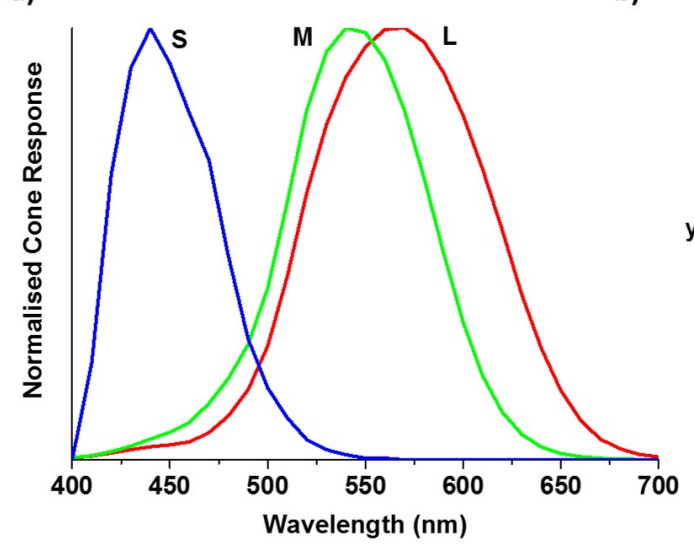

b) CIE 1931

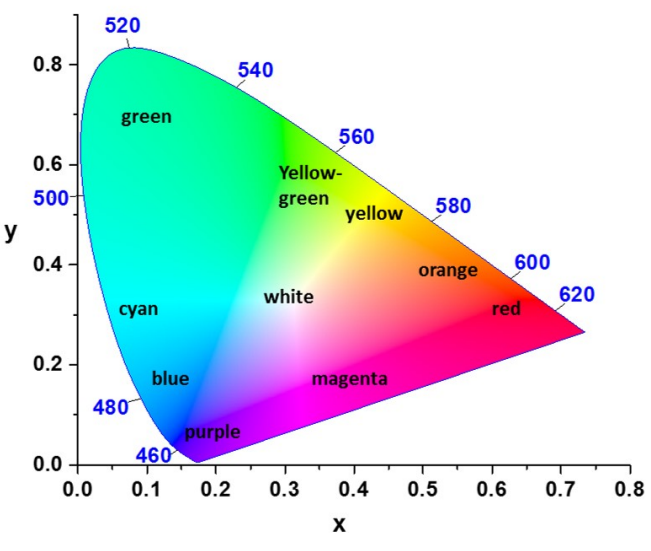

Figure 1.21: a) Spectral sensitivity of human cone cells in short (S), middle (M), and long (L) wavelengths. b) CIE 1931 colour space diagram.

$$
\begin{aligned}
& Y=\lambda \times \bar{y} \times \delta \lambda \\
& Z=\lambda \times \bar{z} \times \delta \lambda
\end{aligned}
$$

Where, $\lambda$ is the wavelength from the emission spectra in $\mathrm{W} / \mathrm{nm} / \mathrm{sr} . \bar{x}, \bar{y}, \bar{z}$ are the colour matching functions. A colour matching function is the numerical description of the chromatic response of human eye, which is the combination of light that seems to produce the same colour to the human eye. It also allows to reproduce the same colour qualities like saturation or hue. The values of $\bar{x}$, $\bar{y}, \bar{z}$ were taken from the literature [161]. 


\section{Chapter 2}

\section{Size and Composition Dependent $\mathrm{CsPbX}_{3}(\mathrm{X}=\mathrm{Cl}, \mathrm{Br}, \mathrm{I})$ Nanocrystals}

Parts of this chapter have been published in the following articles:

Butkus J, Vashishtha P, Chen, K, Gallaher JK, Prasad SK, Metin DZ, Laufersky G, Gaston N, Halpert JE, Hodgkiss, JM. The Evolution of Quantum Confinement in CsPbBr $r_{3}$ Perouskite Nanocrystals. Chemistry of Materials 2017.

Vashishtha P \& Halpert JE. Field-Driven Ion Migration and Color Instability in Red-Emitting Mixed Halide Perouskite Nanocrystal Light-Emitting Diodes. Chemistry of Materials 2017.

\subsection{Introduction}

Semiconducting NCs have proven to be useful materials for optoelectronic applications, such as solar cells, LEDs, and lasers due to their thin linewidth emission spectra, high PL quantum yield and high absorption coefficient $[1,2,162]$. Significant efforts have already been made over last two decades to control the 
shape and size of NCs $[10,163,164]$. Additionally, perovskite materials have drawn tremendous attention due to their promising properties such as high absorption coefficient, halide composition tuneability, long charge carrier lifetimes $(\approx 54 \mu \mathrm{s})$, charge diffusion length $(\approx 37 \mu \mathrm{m})$, and low defect densities $\left(\approx 10^{9}-10^{10} \mathrm{~cm}^{-3}\right)[81-83,165-167]$. Perovskite NCs combine the properties of both perovskites and NCs. To date, researchers have made high quantum yield and tuneable perovskite NCs using methylammonium lead halide (MAPX) and pure inorganic caesium lead halide $\left(\mathrm{CsPbX}_{3}\right)[17,18,168]$. Due to their quantum confined and composition dependent tuneable properties, the NCs of perovskites have proven themselves to be a promising candidates for LEDs and electrical pumped lasers $[21,158,169]$. Lead free perovskite NCs, $\mathrm{CsSnX}_{3}$, were also synthesised but the quantum yield was measured to be only $0.14 \%$ [22] Therefore they found to be very inefficient for any optoelectronic applications.

Quantum confinement in NCs can be estimated with the help of Bohr diameter of bulk material. A Bohr diameter is the diameter of exciton quasiparticles, which is unique for each material. If the material's diameter is larger than the Bohr diameter, then it is in the weak confinement regime. Whereas, if particle dimension is smaller than the Bohr diameter, then it is in the strong confinement regime. However, if the particle dimension is in the region of Bohr diameter, then it is considered to be in the intermediate confinement regime.

Confinement effect is responsible for changing the material's properties due to the constraints on carrier wave function within the NC. This results in the change of material energy levels. The confinement effect has been observed in many types of NCs in the last last two decades [9, 170-172]. To date, one of the best quantum confined NCs are CdSe due to their emission tuneability through the entire visible spectrum [173]. The extensive quantum confinement study has been carried out on CdSe NCs, which shows the size of NCs can be even smaller than their Bohr diameter [174]. All these size tuneability in CdSe NCs make them a promising material for LEDs, solar cells, sensors, optical filters, and lasers [175-179]. However, due to the toxicity of Cd, there are multiple 
barriers for them in the markets of Europe and Japan [180].

This chapter includes the synthesis of highly luminescent $\mathrm{CsPbX}_{3}(\mathrm{X}=\mathrm{Cl}, \mathrm{Br}$, I) perovskite NCs with size and composition dependent band gap by colloidal route. The effect of quantum confinement on the photophysics of the size dependent $\mathrm{CsPbBr}$ NCs was yet to be observed in literature. Therefore, $\mathrm{CsPbBr}_{3}$ NCs were characterised by steady state absorption and PL spectroscopy to understand the quantum confinement in these NCs. They were further characterised by ultrafast transient absorption (TA) spectroscopy by Hodgkiss group at VUW to support the quantum confinement observed by steady state measurements. The halide composition dependent NCs demonstrated the emission tuneability through the entire visible spectrum. These NCs were further used in the preparation of LEDs (Chapter 3).

\subsection{Experimental}

\subsubsection{Synthesis of Size Dependent $\mathrm{CsPbBr}_{3} \mathrm{NCs}$ and Microcrys- talline Film}

\subsubsection{Materials}

All the chemicals were used without any processing. Caesium carbonate $(99 \%$, Aldrich), lead(II) bromide (99.999\%, Aldrich), oleylamine (98\%, Aldrich), oleic Acid ( 99\%, Fluka), caesium bromide (99.999\% Aldrich), 1-Octadecene (90\%, Aldrich), caesium bromide (99\%, Aldrich), dimethyl sulfoxide (DMSO) (99\% anhydrous, Aldrich), and acetonitrile (99\% anhydrous, Aldrich). 


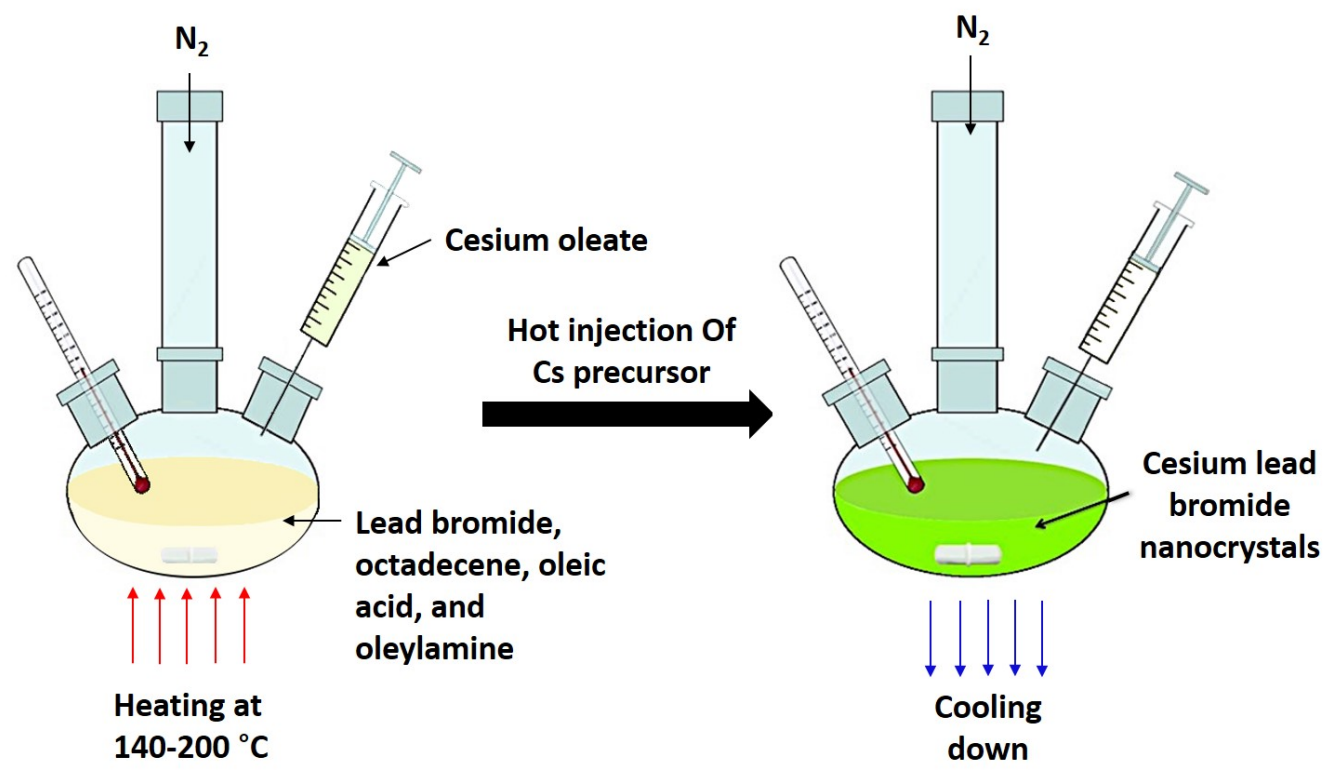

Figure 2.1: A diagram for the hot-injection synthesis method of $\mathrm{Cs} \mathrm{PbBr}_{3} \mathrm{NCs}$.

\subsubsection{Method}

The synthesis method was adapted from those reported by Protesescu et al [17]. In this method, Cs-oleate was used as a caesium source; $\mathrm{PbBr}_{2}$ was used as a lead and halide source. Oleylamine and oleic acid were used as capping agents. It was possible to tune the emission spectra and apparent band gap energy by changing the size of quantum dots. The synthesis procedure is divided into two parts: 1) synthesis of Cs-oleate precursor and 2) synthesis of perovskite NC $\left.(\mathrm{CsPbBr})_{3}\right)$.

Synthesis of Caesium Oleate: Caesium oleate was synthesised by loading 0.4 gm caesium carbonate (99\%, Aldrich), $15 \mathrm{~mL}$ ODE (90\%, Aldrich) and $1.2 \mathrm{~mL}$ oleic acid (99\%, Fluka) into a $50 \mathrm{~mL} 3$-neck flask. The reaction mixture was degassed for $1 \mathrm{~h}$ at $120^{\circ} \mathrm{C}$ under vacuum and then temperature was raised to $150{ }^{\circ} \mathrm{C}$ and heated for $30 \mathrm{~min}$ for the $\mathrm{Cs}_{2} \mathrm{CO}_{3}$ to react with the oleic acid. 
Synthesis of NCs: $5 \mathrm{~mL} \mathrm{ODE}$ and $0.18 \mathrm{mmol} \mathrm{PbBr}_{2}$ were added to a $50 \mathrm{~mL} \mathrm{3-}$ neck flask and degassed for $1 \mathrm{~h}$ under vacuum at $120^{\circ} \mathrm{C}$. Simultaneously, 0.5 $\mathrm{mL}$ oleylamine and $0.5 \mathrm{~mL}$ oleic acid were degassed under the same conditions in a separate vial and then added to the reaction mixture under $\mathrm{N}_{2}$. It should be noted that Oleylamine and oleic acid were always stored in a refrigerator below $6{ }^{\circ} \mathrm{C}$ and degassed as described above in order to remove water or aqueous solution. The reaction temperature was increased to $140{ }^{\circ} \mathrm{C}$ and held for $30 \mathrm{~min}$ in order to dissolve all reactants. $0.4 \mathrm{~mL}$ Cs-oleate was injected quickly into the reaction flask at a particular temperature $\left(140-200{ }^{\circ} \mathrm{C}\right)$. The reaction flask was cooled down after few seconds (see Table 2.1) [17].

Purification of NCs: Perovskite NCs are very hygroscopic therefore precautions needed to be taken to exclude water in the reaction. To remove the excess ligands and organics from NCs surface, they need to be washed properly by solvent/anti-solvent method (Section 1.2.5.3). NCs are dispersed in a good solvent and then an anti-solvent (bad solvent) is added which mixes with the solvent but does not mix with the particles causing aggregation. Then, the solution is centrifuged to aggregate the NCs and this process needs to be repeated a number of times to get rid of excess organic. Choosing the right anti-solvent for washing the NCs, which does not damage their surface, is a very important step. The entire purification process was carried out in an anhydrous solvent in a $\mathrm{N}_{2}$ glove box. Several purification methods were carried out. The NCs solutions were dissolved in $5 \mathrm{~mL}$ of hexane after which $10 \mathrm{~mL}$ of ethanol was added as an antisolvent. The solution was centrifuged at 10000 RPM and the process was repeated two times. Finally, the NCs were re-dispersed in hexane. However, due to the presence of $\mathrm{OH}$ groups, ethanol even removed the necessary ligands from the particle surface, resulting in agglomeration of the particles. Different ratios of ethanol/hexane were investigated but there was no significant improvement. Additionally, the emission of NCs appeared to decrease after each purification step. Acetonitrile is another anti-solvent which does not have $\mathrm{OH}$ group. The next purification approach was carried out by using acetonitrile. The growth solution was centrifuged at 10,000 RPM for $10 \mathrm{~min}$, the 
supernatant was then discarded and the precipitate was redispersed in $6 \mathrm{~mL}$ of anhydrous toluene and $2 \mathrm{~mL}$ of anhydrous acetonitrile was added as an antisolvent. The resulting solution was centrifuged again at 10000 RPM for $8 \mathrm{~min}$. This cycle was repeated one more time. After discarding the supernatant, the precipitate was dried under vacuum for $5 \mathrm{~min}$ and then re-dispersed in anhydrous hexane with $12 \mathrm{mg} / \mathrm{mL}$ concentration. NCs were filtered through $0.2 \mu \mathrm{m}$ PTFE filter before the fabrication of devices.

Fabrication of $\mathrm{CsPbBr}_{3}$ Microcrystalline Film: $\mathrm{CsPbBr}_{3}$ microcrystalline films were prepared by dissolving the equimolar ratio of $\mathrm{CsBr}$ and $\mathrm{PbBr}_{2}$ in dimethyl sulfoxide (DMSO) followed by spin coating on a $\mathrm{TiO}_{2}$ coated glass substrate at $2000 \mathrm{RPM}$ for $60 \mathrm{~s}$. The film was annealed at $100^{\circ} \mathrm{C}$ in a nitrogen glove box in order to induce a crystallisation.

\subsubsection{Synthesis of Composition Dependent Perovskite NCs}

The caesium oleate was synthesised by the same method as described in the Section 2.2.1.2. In this method, $0.5 \mathrm{~mL}$ of ODE, $\mathrm{x} \mathrm{mmol} \mathrm{of} \mathrm{PbBr}_{2}$, and (0.18-x) mmol of $\mathrm{PbI}_{2}$ or $\mathrm{PbCl}_{2}$ were added to a $50 \mathrm{~mL}$ 3-neck flask and degassed for $1 \mathrm{~h}$ under vacuum at $120^{\circ} \mathrm{C}$ (Table 2.3). Simultaneously, $0.5 \mathrm{~mL}$ oleylamine and 0.5 $\mathrm{mL}$ oleic acid were degassed under the same conditions in a separate vial and then added to the reaction mixture under nitrogen. The reaction temperature was increased to $140{ }^{\circ} \mathrm{C}$ for $30 \mathrm{mins}$ in order to dissolve all of the reactants. 0.4 $\mathrm{mL}$ Cs-oleate was injected quickly into the reaction flask at $170^{\circ} \mathrm{C}$. The reaction flask was cooled down after 5-20 s [17]. The reaction conditions were optimised in order to achieve the desired particles size. The purification process was similar to pure $\mathrm{CsPbBr}_{3}$ samples Section 2.2.1.2. In that case, The NCs were purified using acetonitrile as an anti-solvent and toluene as a solvent. The final precipitate of NCs was re-dispersed in hexane for further characterisation and device fabrication. 


\subsubsection{Synthesis of $\mathrm{CsPbX}_{3} \mathrm{NWs}$}

The synthesis of NWs is similar to NCs with slight change in the reaction conditions. Therefore, the amount of oleylamine was increased to $2 \mathrm{~mL}$ and the growth time was extended to $12 \mathrm{~h}$ for $\mathrm{CsPbBr}_{3}$ and $6 \mathrm{~h}$ for $\mathrm{CsPbI}_{3}$. The growth solution was centrifuged at $10000 \mathrm{RPM}$ for $10 \mathrm{~min}$ followed by washing in toluene and an acetonitrile mixture at 10000 RPM up to two cycles (Section 2.2.1.2). The precipitate of the NWs were dispersed in hexane for further characterisation.

\subsection{Results and Discussion}

Several characterisations have been performed to understand the reaction mechanism, morphology, and optical properties. TEM and XRD measurements were carried out to observe the morphology of these NCs. However, steady state UVVisible, PL, and ultrafast transient absorption spectroscopy provided details on the optical properties. Ultrafast transient measurements were measured and analysed by Dr Justinas Butkus under the guidance of Prof Justin Hodgkiss at Victoria University of Wellington.

\subsubsection{Morphological Characterisation on Size Controlled NCs}

The NCs washed by ethanol/hexane appeared to agglomerate and lose their luminescence. The Figure 2.22a suggests that the particles were not even washed properly and still had the organics around. Both ethanol and acetone were not proven to be good solvents for hydrophobic NCs. Later on, the purification step was improvised with the acetonitrile/toluene. The TEM image (figure 2.2b) shows properly washed monodispersed cubic $\mathrm{NCs}$ of $\mathrm{CsPbBr}_{3}$ with no damage to their surface. 


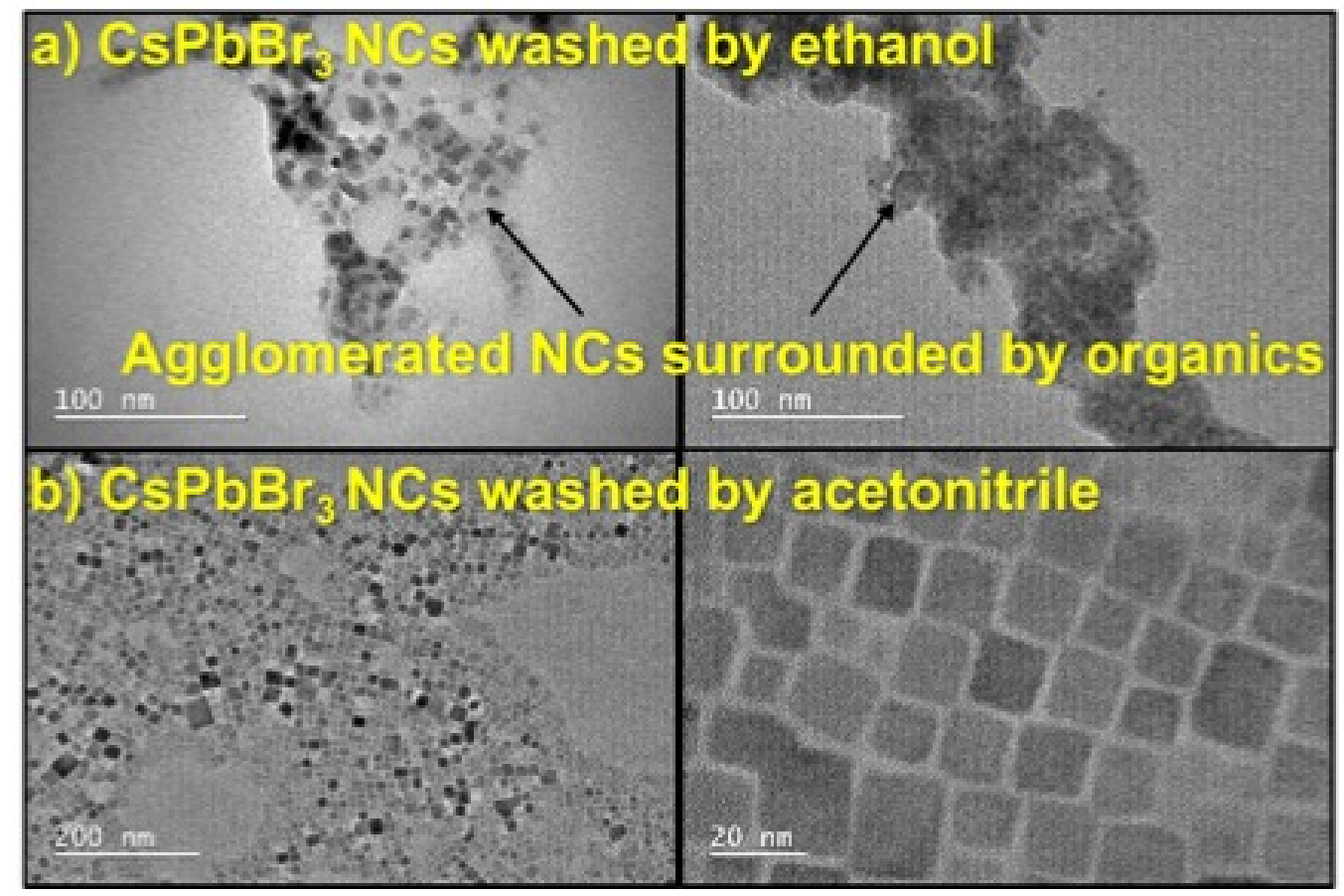

Figure 2.2: TEM micrographs of $C s \mathrm{PbBr}_{3}$ NCs washed by a) ethanol and b) acetonitrile. The TEM samples were prepared by drop-casting hexane NCs solution on a Cu-200 mesh on formvar grid.

To understand the reaction rate, the growth time was extended to $2 \mathrm{~min}$. It was found that some large sheets appeared along with well dispersed NCs due to the fusion of NCs with their neighbouring particles [181]. Figure 2.3 shows the TEM images of larger nanosheets which were formed during this fusion process. In order to control the reaction kinetics, the growth time should be limited to $30 \mathrm{~s}$.

Three different samples of $\mathrm{CsPbBr}_{3}$ NCs were synthesised with the average particle sizes of 4.1, 7.3, and $8.6 \mathrm{~nm}$. Size of the particles was measured by ImageJ and then the histograms were created for each TEM image. Figure 2.4 shows the TEM image of these NCs and the histogram on top of each image confirms the average particle size with standard deviation and shows the monodispersity in NCs. The size of the particles was tuned by changing the reaction time and temperature. Table 2.1 shows the reaction conditions for individual NCs 


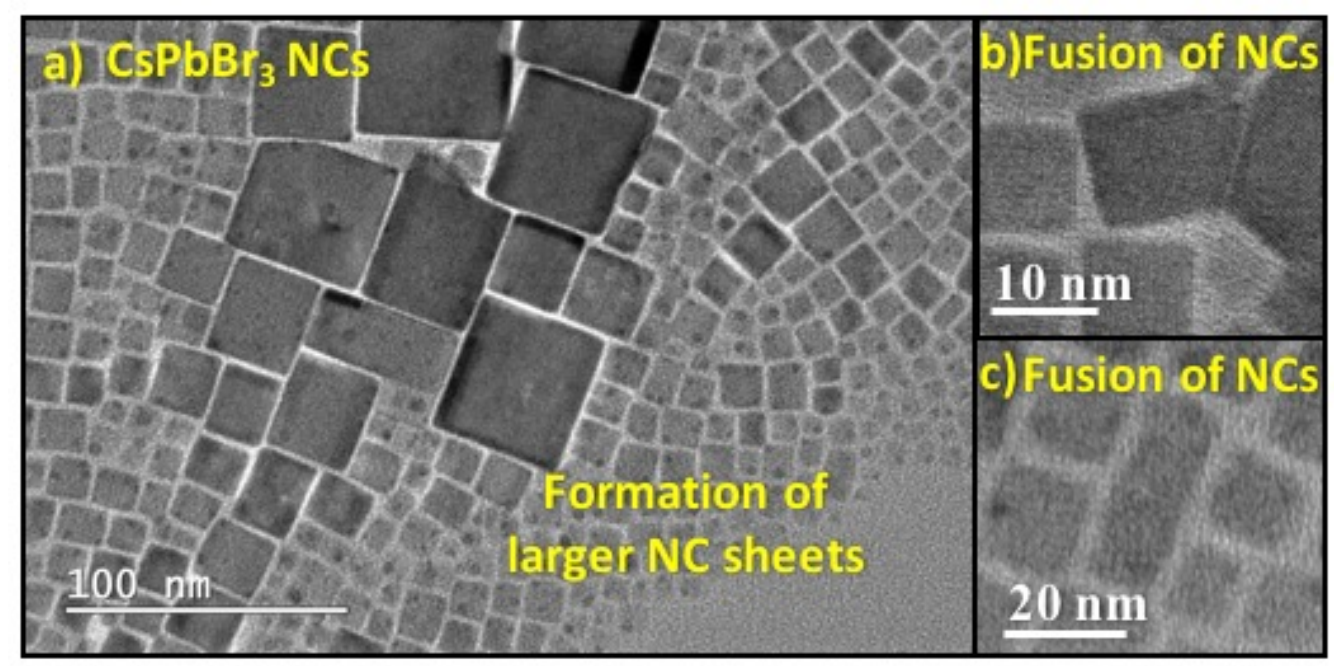

Figure 2.3: TEM micrographs of CsPbX $X_{3}$ NCs shows the fusion of NCs into their neighbouring particles and forming larger nanosheets. The TEM samples were prepared by drop-casting hexane NCs solution on a Cu-200 mesh on formvar grid.

sample, which describes the growth time and reaction temperature required for each sample.

The nucleation and growth kinetics of the reaction were found to be very fast. Therefore, high reaction temperature or longer time led to larger particle size. Controlled hot-injection reaction ensured the monodispersity of the NCs which can be seen in the TEM micrographs (Figure 2.4). The high resolution TEM image (Figure 2.4b) shows the crystalline lattice fringes. The lattice fringes were used to create the fast Fourier transform (FFT) using ImageJ, which is shown in figure 2.4c. This FFT was compared with the FFT of standard crystal structure taken from X'Pert HighScore (Ref. Code- 04-017-4526) in CrystalMaker to confirm the perovskite crystal structure.

The XRD samples were prepared by drop casting the concentrated NCs solution (in hexane) on to a clean glass slide. The microcrystalline film was prepared by spin coating a $100 \mathrm{~nm}$ thick layer on to a $\mathrm{TiO}_{2}$ coated glass substrate. 


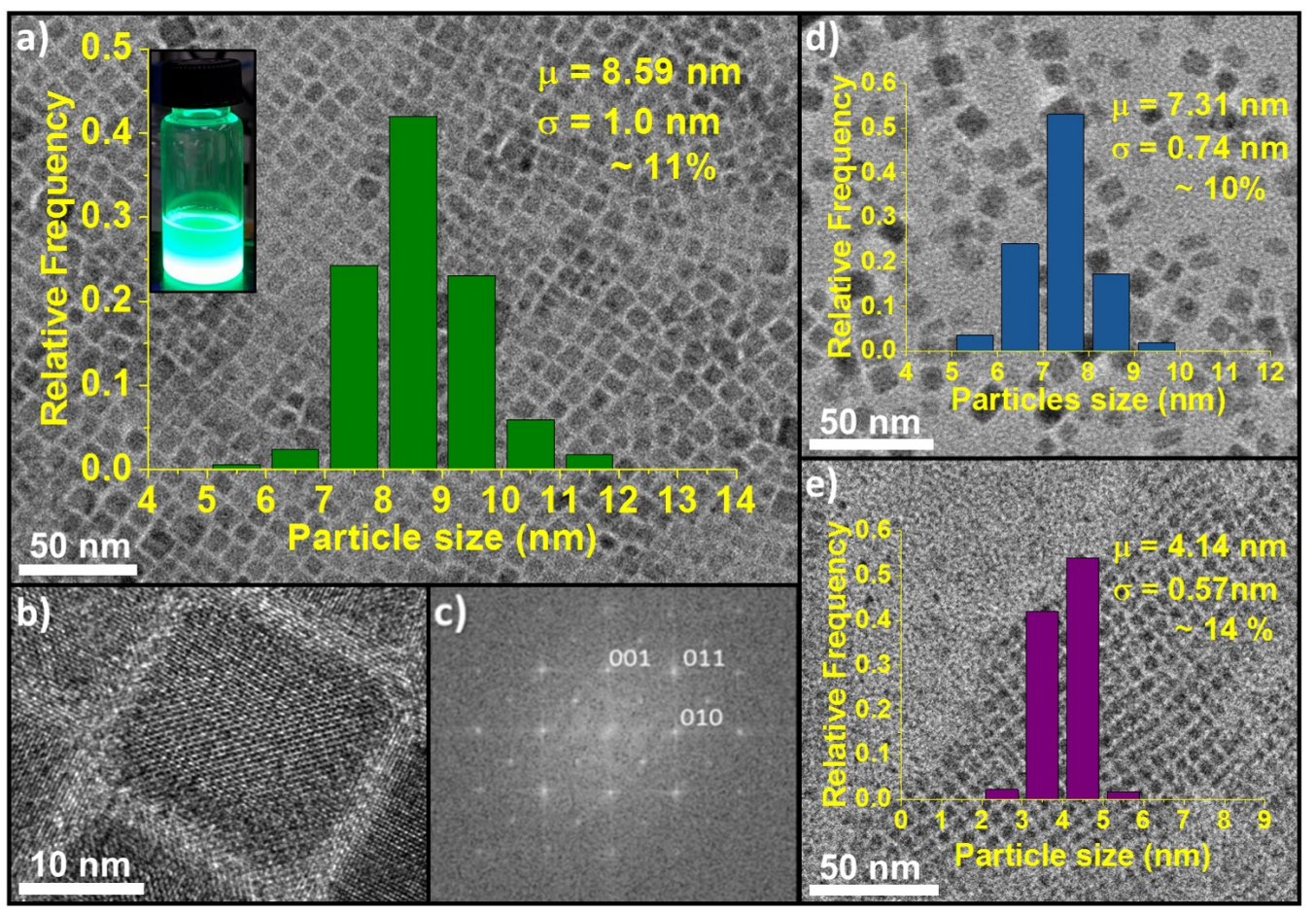

Figure 2.4: (a,d,e) High resolution transmission electron micrographs (HRTEMs) (b) imaging of lattice planes of a $\mathrm{CsPbr}_{3} \mathrm{NCs}$ and (c) a fast Fourier transform (FFT) image from (b) showing the crystallinity of the NCs. A photograph of NC-8.6 sample in hexane solvent under UV light (overlaid a), Histograms (overlaid a,d,e) of particle size, measured as mean length of the edge of the cube, are also included for three samples with average edge length in Table 2.1. TEM samples were prepared by drop-casting hexane NCs solution on a Cu-200 mesh on formvar grid. Copyright 2017 American Chemical Society.

Table 2.1: A table of synthesis conditions for the $\mathrm{CsPbr}_{3} \mathrm{NCs}$ along with the resultant particle size.

\begin{tabular}{|c|c|c|}
\hline Sample Name & Growth Time $(\mathbf{s})$ & Temperature $\left({ }^{\circ} \mathbf{C}\right)$ \\
\hline NC-4.1 & $4-5$ & 140 \\
NC-7.3 & $4-5$ & 165 \\
NC-8.6 & $8-10$ & 175 \\
\hline
\end{tabular}




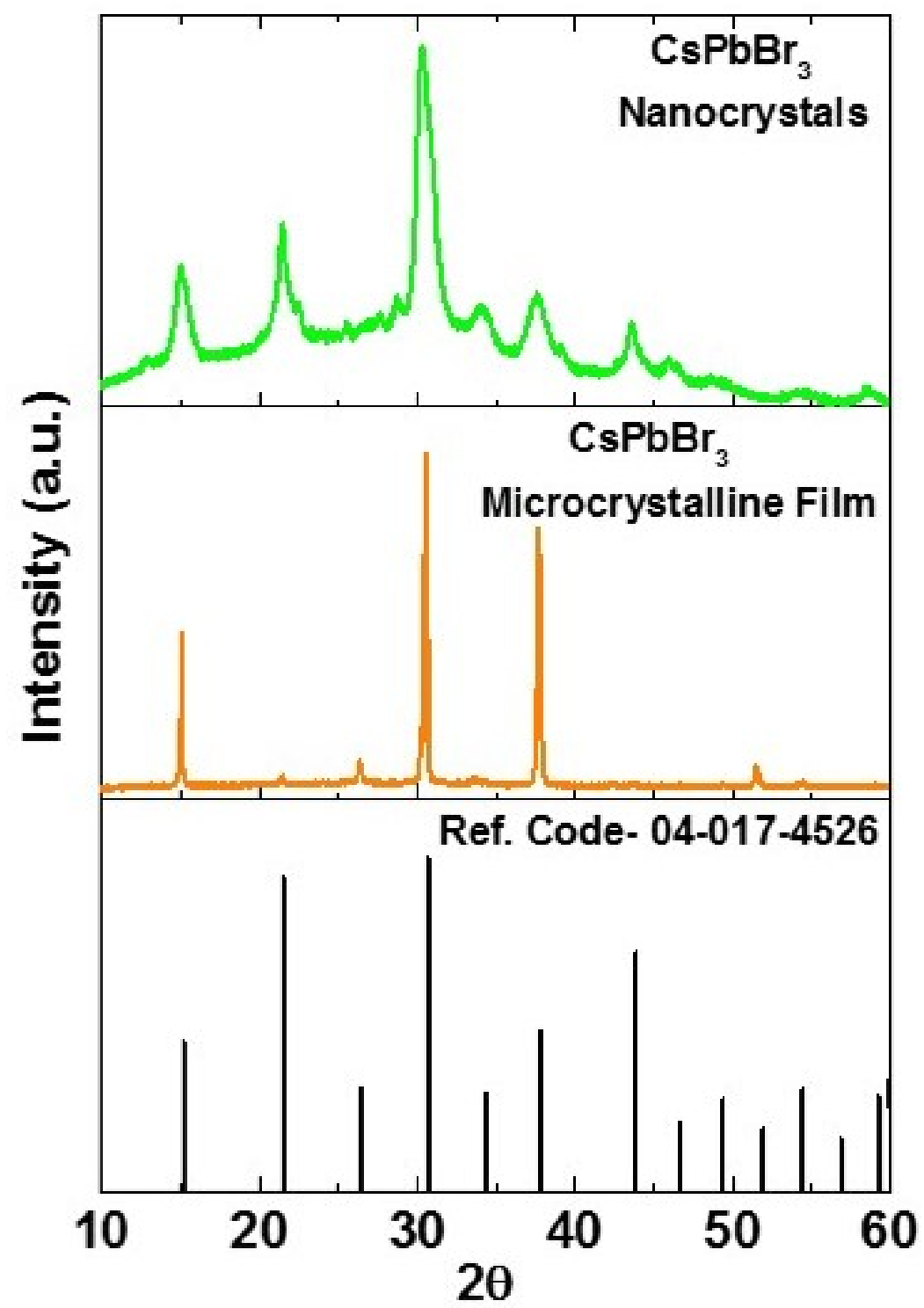

Figure 2.5: XRD spectra of $C s P b B r_{3}$ NCs and microcrystalline film. XRD patterns were recorded by a PANalytical X-Ray diffractometer using $\mathrm{Cu}$-Ka radiation, with an operating voltage of $45 \mathrm{kV}$ and current of $40 \mathrm{~mA}$. The standard spectra of $\mathrm{CsPbBr}_{3}$ was taken from PANalytical X'Pert HighScore Plus with the reference code 04-017-4526. 
XRD spectra were taken with a PANalytical X-Ray diffractometer using $\mathrm{Cu}-\mathrm{K} \alpha$ radiation on a spinner stage, with an operating voltage of $45 \mathrm{kV}$ and current of $40 \mathrm{~mA}$. XRD peaks of NCs were found to be broadened compared to the microcrystalline peaks according to the Scherrer equation (1.39). The average crystalline size of microcrystalline sample was estimated to be $\approx 51 \mathrm{~nm}$ using Scherrer equation (1.39). XRD spectra of both NCs and microcrystalline films were matched to the index spectra using PANalytical X'Pert HighScore Plus software data file (Ref. Code: 04-017-4526). The matching of lattice planes in fast Fourier transform (FFT) with cubic perovskite standard XRD crystal structure (Figure 2.5) further confirms the formation of perovskite material [182].

\subsubsection{Optical Spectroscopy on Size Controlled $\mathrm{CsPbBr}_{3} \mathrm{NCs}$}

Optical spectroscopy can give details of the material properties and can help to understand the quantum confinement effect. Each NCs sample was diluted in hexane for the UV-Vis and PL measurements. A Cary $50 \mathrm{UV}$-Visible spectrophotometer was used to do the UV-Vis spectroscopy and a Horiba Fluorolog3 Spectrometer were used for the PL measurement. NCs were measured in the form of solution and a $10 \mathrm{~mm}$ quartz cuvette with pure hexane were used to create the baseline. For the microcrystalline film, a blank $\mathrm{TiO}_{2}$ coated substrate was used to create the baseline.

The absorption and PL measurements were carried out on the $\mathrm{CsPbr}_{3}$ microcrystalline films and each NC sample. We observed the change in the optical properties of the materials as the size changes. The PL peak was blue shifted with decreasing size as per the confinement effect. The Tauc plot also supports the blue shifting of emission peak as the estimated band gap increased with the decrease in particle size (Figure 2.6b). The bandgap of smallest NCs shifted about $5.2 \%$ of its bulk value, which is one of the appearance of confinement effect (Table 2.2). Although, the blue shift of the PL matches with the band gap, this evidence is still not enough to prove the confinement effect. So, we used 

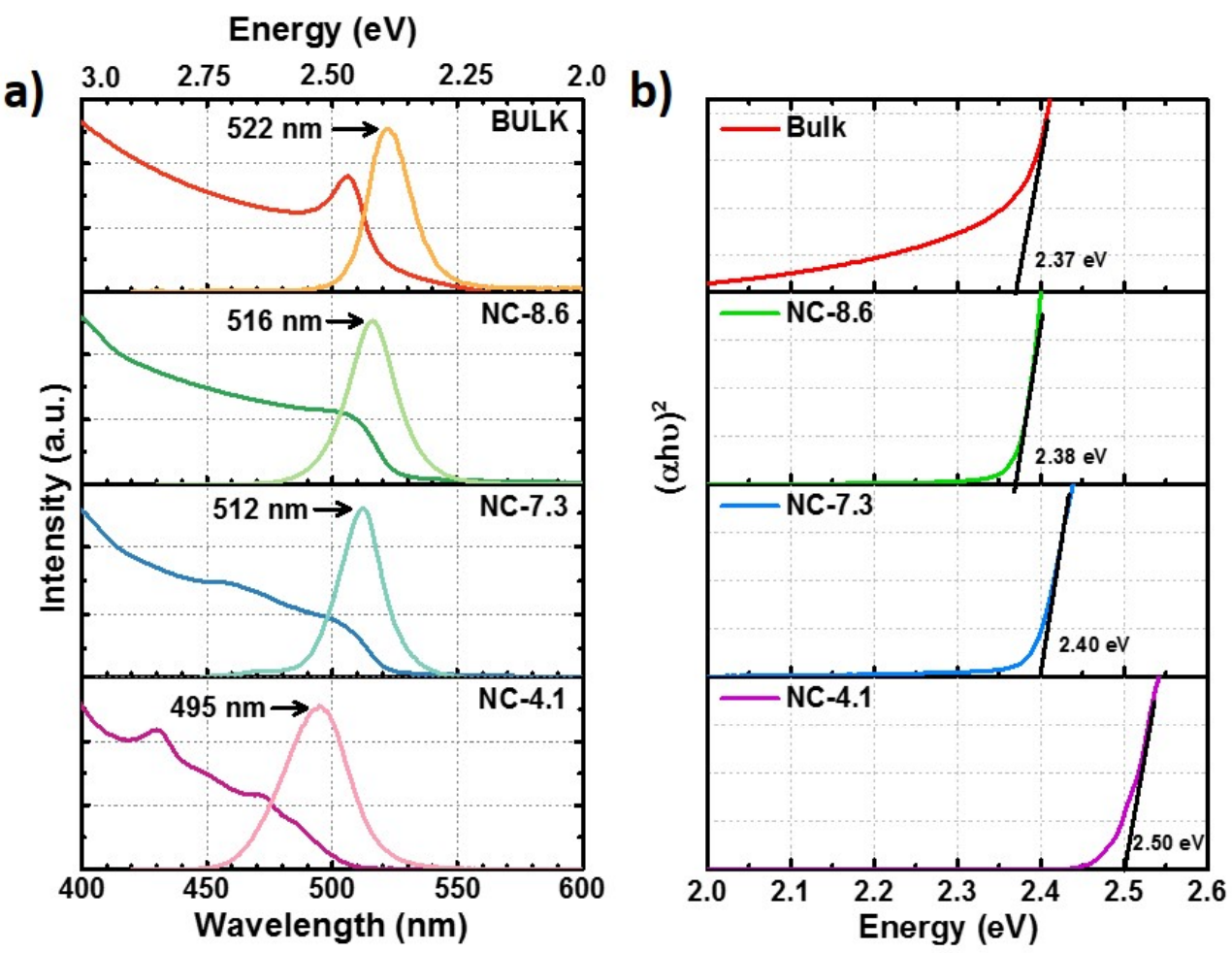

Figure 2.6: a) Absorbance (broad peak, left) and PL (Gaussian peak, right) spectra of the four $\mathrm{CsPbBr}$ samples showing emission peaks at $522 \mathrm{~nm}$ (red curves), $516 \mathrm{~nm}$ (green curves), $512 \mathrm{~nm}$ (blue curves), and $495 \mathrm{~nm}$ (purple curves) for the different samples b)Direct bandgap Tauc plots of the same samples to calculate the band gap of each sample.

the absorption edge and the PL peak to estimate the Stokes shift, which is basically the energy difference between lowest energy absorption edge and PL peak position. We found that the estimated Stokes shift for the NCs increased as the size decreased. The smaller NCs tend to have higher crystal disorder due to their higher surface area. Therefore, the smallest NCs have the largest Stokes shift. This agrees with our previous conclusion of the blue shifting of emission peak with decreasing the particle size. The estimated Stokes shift for microcrystalline films was $0.070 \mathrm{eV}$, which is larger than the shift calculated in even the largest NCs. But possibly, it is due to the thermalisation of the material 
[183]. The higher energy absorption peaks were also observed in smallest NCs (NC-4.1). These higher energy absorption peaks correlate to the divulgation of energy level splitting due to the quantum confinement effect [184].

To compare the accuracy of experimental band gap, we calculated the theoretical direct band gap values at different particle sizes from the following form of the Brus equation $[185,186]$

$$
E=E+\frac{\hbar^{2} \pi^{2} \mu_{e x}^{*}}{2 r^{2}}-\frac{1.8 e^{2}}{4 \pi \epsilon_{r} \epsilon_{0} r}
$$

Where, $\mu_{e x}^{*}$ is the exciton reduced mass and can be calculated using the following equation:

$$
\mu_{e x}^{*}=\frac{1}{m_{e}^{*}}+\frac{1}{m_{h}^{*}}
$$

Where, $\mathrm{r}$ is the radius of particle, $\mathrm{m}_{e}^{*}$ is effective mass of electrons, $m_{h}^{*}$ is the effective mass of holes, $\varepsilon_{\mathrm{r}}$ is the relative permittivity, and $\mathrm{E}_{\mathrm{g}}$ is the bulk band gap. Figure 2.7 shows the experimental and theoretical plot of band gap against the quantum confinement regime [17]. Both band gaps align with each other reasonably well. According to figure 2.6, NC-8.6 and NC-7.3 samples fall under

Table 2.2: NCs size estimate, optical characterisation measurement data, and Stokes shift

\begin{tabular}{|c|c|c|c|c|}
\hline Measurements & Films & NC-8.6 & NC-7.3 & NC-4.1 \\
\hline Edge length (nm) & 51 & $8.59 \pm 1.0$ & $7.32 \pm 0.74$ & $4.14 \pm 0.57$ \\
PL peak (nm) & 522 & 516 & 512 & 495 \\
PL FWHM (nm) & 20 & 22 & 21 & 32 \\
BG (eV) & 2.37 & 2.38 & 2.40 & 2.50 \\
Stokes shift (eV) & 0.070 & 0.022 & 0.027 & 0.090 \\
\hline
\end{tabular}




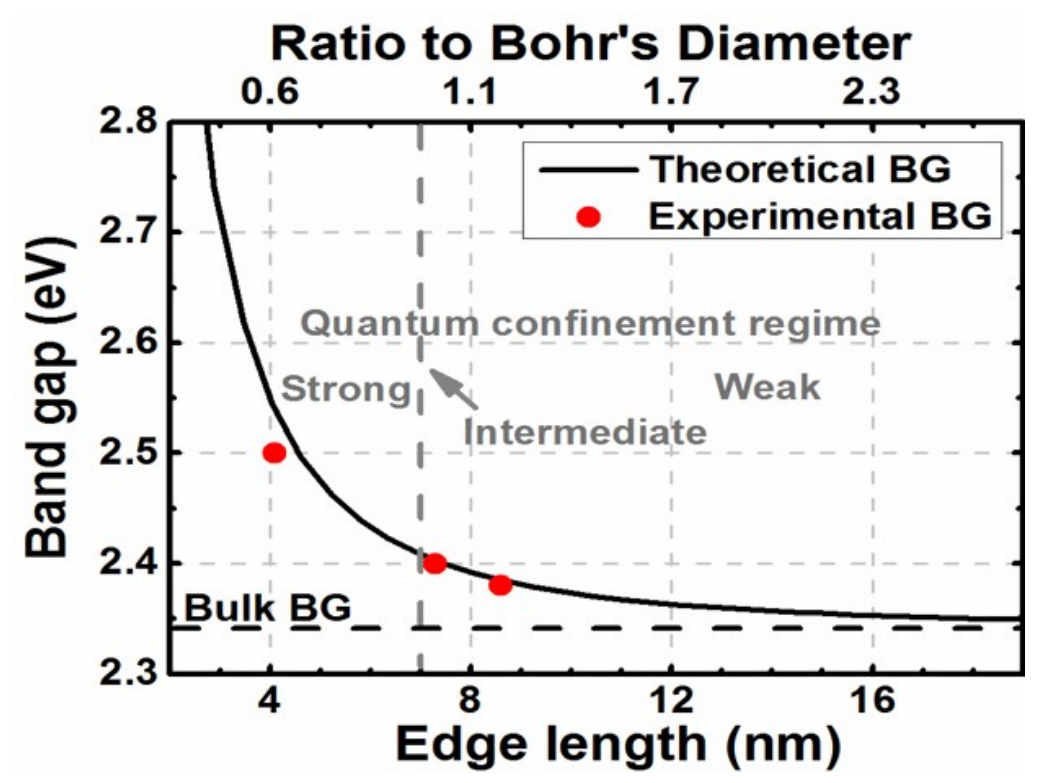

Figure 2.7: Experimental versus theoretical (effective mass approximation) size dependence of the band gap energy with quantum confinement regimes noted in relation to the Bohr diameter $(\mathrm{db}=7 \mathrm{~nm})$. Copyright 2017 American Chemical Society (Courtesy of Dr Justinas Butkus).

weak and intermediate confinement regime. However, for the NC-4.1 samples, both band gaps lie under strong confinement regime, which supports the formation of the multiple absorption edges in this sample and therefore the formation of discrete energy levels.

In order to further support our observation on the quantum confinement effect in these NCs, the ultrafast transient absorbance (TA) characterisations were carried out by Dr Justinas Butkus under the guidance of Prof Justin Hodgkiss. The TA spectra of the largest and smallest NCs is shown in Figure 2.7. Figure $2.8 \mathrm{~b}$ shows the TA spectra of NC-8.6 and NC-4.1, which gives a clear indication of ground state bleach (GSB). The monomodal GSB shape of the largest NCs is the characteristic of Burstein-Moss effect, which is basically the increment in the apparent band gap of semiconductors due to a higher energy shift in absorption edge since all the states close to the conduction band becomes populated (Figure 2.8a) [187]. The broad negative differential transmission fea- 
a) Burstein-Moss effect in perovskite

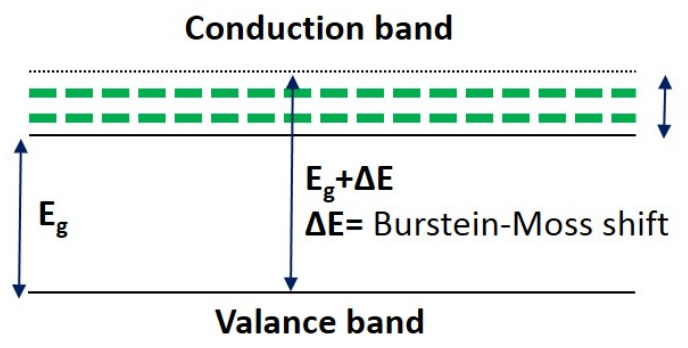

b)

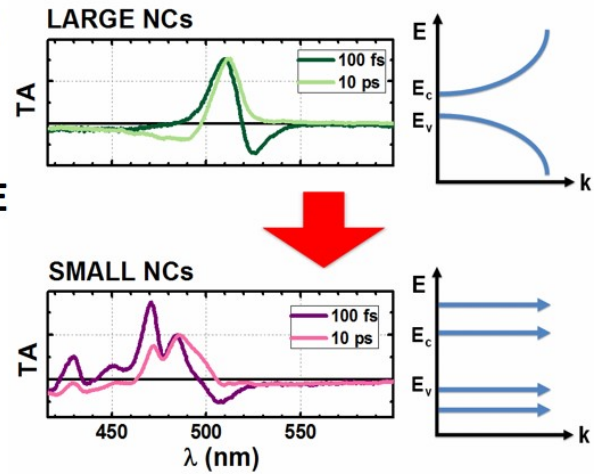

Figure 2.8: a) A schematic diagram of Burstein-Moss effect in semiconductors, $b$ ) transient absorbance (TA) spectra of NC-8.6 and NC 4.1 NCs with corresponding development of molecular energy state from continuous to molecular like states (Courtesy of Dr Justinas Butkus).

ture above the band gap was correlated to scattering for NCs solution due to the photorefractive effect [187]. Both samples shows several similarities including GSB feature, band gap renormalization, and a negative differential transmission feature in their TA spectral profile. However, the GSB is blue shifted in smaller particles (figure 2.8b) like steady state absorption spectra (figure 2.6a). The other major difference is the multimodal GSB feature which is very pronounced in NC-4.1 sample. Therefore, the smaller NCs resembles the emergence of molecular like states, while the larger NCs and microcrystalline film indicates the continuous bands. This shows that NC-8.6 is not in the strong confinement regime, but in the weak confinement regime. Thus, the TA measurements also suggests the strong confinement in the smallest sample and the weak confinement in the larger NCs. TA measurements were also conducted on other samples but the details can be found in our recent report, as this work was done in collaboration with Prof Justin Hodgkiss [188]

In conclusion, we found a similar trend in all of the samples. The increment in band gap as the particle size decreased, the blue shifted emission peak with the particle size, increment in Stokes shift as the particle size decreased, the origin of discrete energy levels observed by the higher energy absorption spectra in 
NC-4.1, and also the multimodal GSB in NC-4.1 demonstrated the quantum confinement effect in the material.

\subsubsection{Morphological Characterisation on Halide Composition Dependent Perovskite NCs}

Due to the limitations of the confinement effect, the emission can be only tuned by $\pm 15 \mathrm{~nm}$ in perovskite NCs as observed in previous section. Therefore,the halide composition tuning is required to cover the entire visible spectra. $\mathrm{CsPbBr}_{3-\mathrm{x}} \mathrm{I}_{\mathrm{x}}$ and $\mathrm{CsPbBr}_{3-\mathrm{x}} \mathrm{Cl}_{\mathrm{x}}(\mathrm{x}=0-3)$ NCs were synthesised by loading the different ratio of halides to achieve the desire emission spectra. Table 2.3 shows the ratio of halide composition with their respective PL peaks.

Table 2.3: Loading ratio for Halide composition tuned NCs and their respective PL peak.

\begin{tabular}{|c|c|c|}
\hline Sample Name & Formula equivalent of Br (3-x) in NCs & PL peak (nm) \\
\hline B499 & 2.25 & 492 \\
G518 & 3.00 & 516 \\
O558 & 1.88 & 558 \\
R607 & 1.375 & 603 \\
R621 & 1.25 & 621 \\
R636 & 0.875 & 632 \\
R642 & 0.75 & 640 \\
R653 & 0.625 & 651 \\
R665 & 0.50 & 667 \\
R671 & 0.375 & 673 \\
R688 & 0.28 & 683 \\
\hline
\end{tabular}

In this table, name of the samples are labeled with their respective emission 
colours. For example, O558 emits in the orange spectral region and R621 emits the red spectral region. Incorporation of iodide shifted the emission towards higher wavelength and incorporation of bromide moved it towards the lower wavelength. Therefore composition tuning in the NCs was able to cover the entire visible spectrum by altering the $\mathrm{I} / \mathrm{Br}$ and $\mathrm{Br} / \mathrm{Cl}$ ratio. Figure 2.9 shows the TEM micrographs of these mixed halide NCs, which represent their cubical shape. NCs edge dimension was between 10-18 nm, which was calculated from their TEM images by using ImageJ software. $\mathrm{CsPbX}_{3}$ also crystallises in the orthorhombic phase but for the optoelectronic application, only the cubic phase is useful. The bulk $\mathrm{CsPb}_{3}$ is unstable at room temperature and the orthorhombic phase transition occurs below $320^{\circ} \mathrm{C}$ [189]. Whereas, the bulk $\mathrm{CsPbBr}_{3}$ was found to be more stable and therefore the mixed halide was proven to be more stable than pure $\mathrm{CsPbI}_{3}$. However, even in the $\mathrm{CsPbr}_{2.0} \mathrm{I}_{1.0}$, the phase transition occurred at $100^{\circ} \mathrm{C}$ [189] which was suitable to stabilise the crystal but was

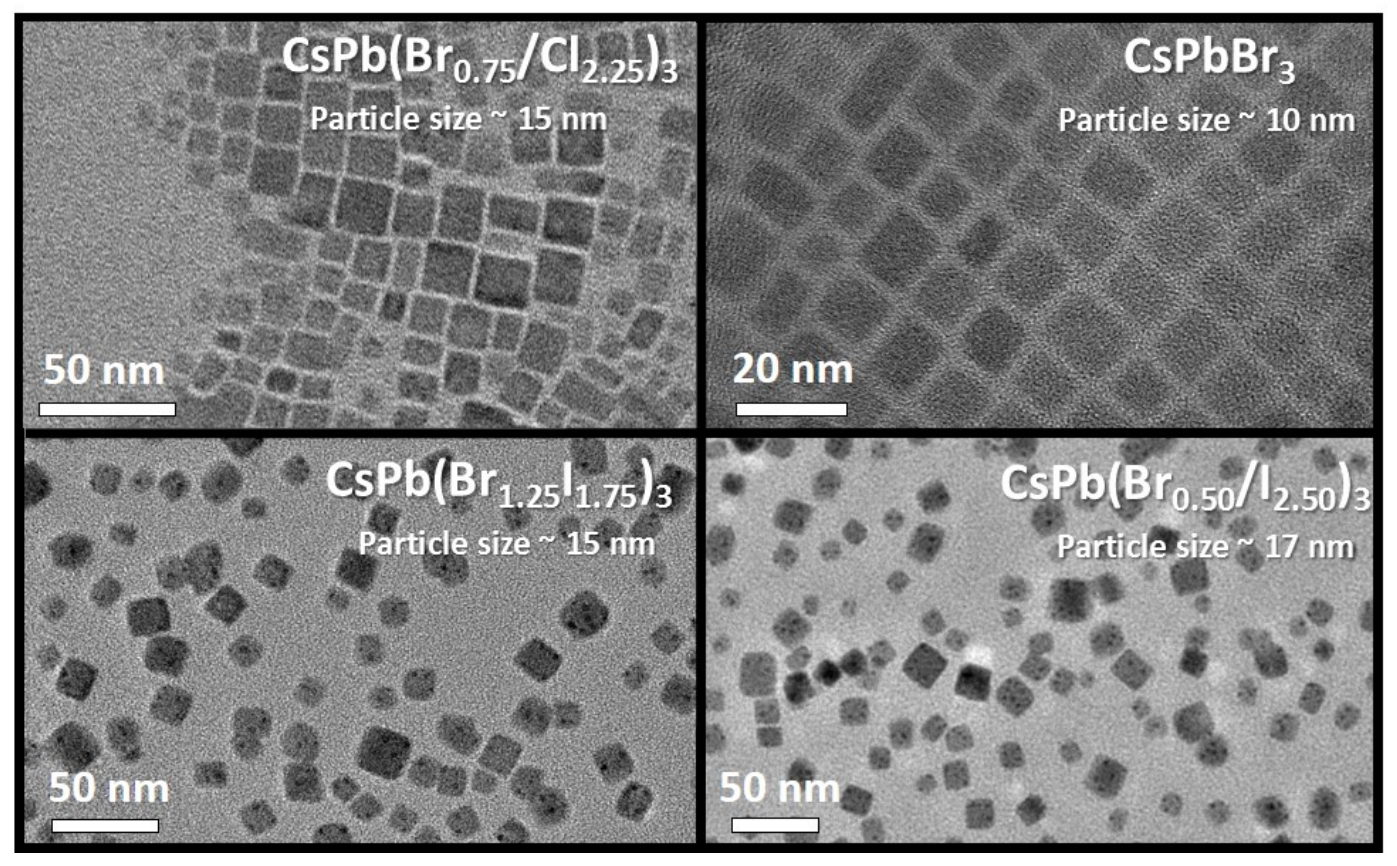

Figure 2.9: TEM micrographs of mixed halide perovskite NCs. The average edge length of the particles are between 10-18 $\mathrm{nm}$ and the samples were prepared by drop-casting hexane NCs solution on a Cu-200 mesh on formvar grid. 
not desirable to produce emission wavelength above $600 \mathrm{~nm}$.

Figure 2.10 shows the NCs hexane suspension under UV lamp. The emission from these NCs were very sharp and the quantum yield was measured between $50-68 \%$. This proves that $\mathrm{CsPbX}_{3}$ NCs are promising materials for LEDs. Due to the suitable ligands, the NCs of these materials demonstrated the phase stability in cubic phase at room temperature but they were still not very stable in ambient condition and were always stored in the nitrogen glove box. The softer basic nature of iodide forms weaker interaction between the amine ligands and halide resulting in the phase transition of cubic $\mathrm{CsPbI}_{3}$ to the orthorhombic phase [190].

The crystal structure of these NCs were confirmed by the XRD measurements (figure 2.11). The standard XRD spectra of orthorhombic and cubic phase is shown in Figure 2.11 for references. All of the peaks in the spectra are labelled with their corresponding literature data. Both the XRD spectra were matched

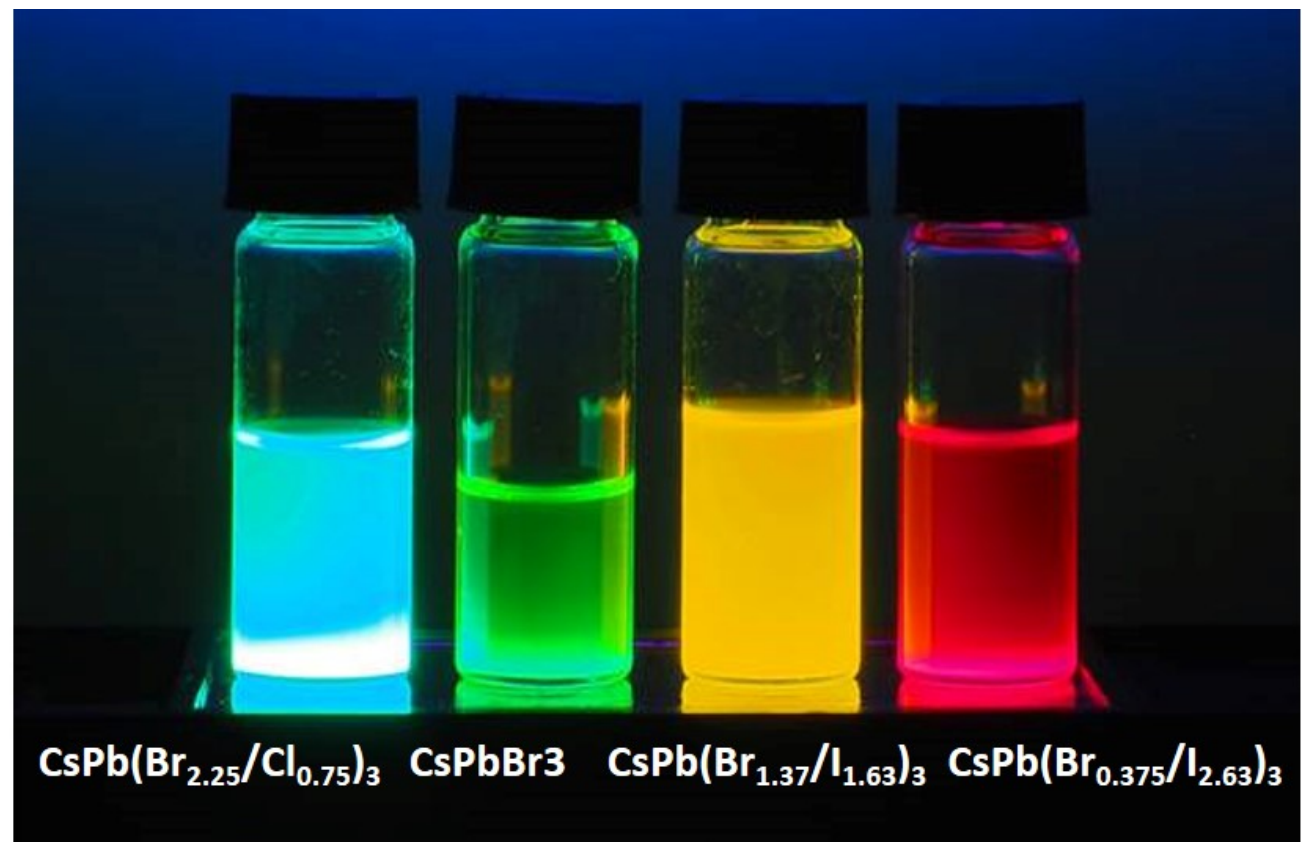

Figure 2.10: A photograph of Mixed halide NCs in hexane under UV lamp with radiation focused at $365 \mathrm{~nm}$. NCs show wide colour gamut from blue to the red. 


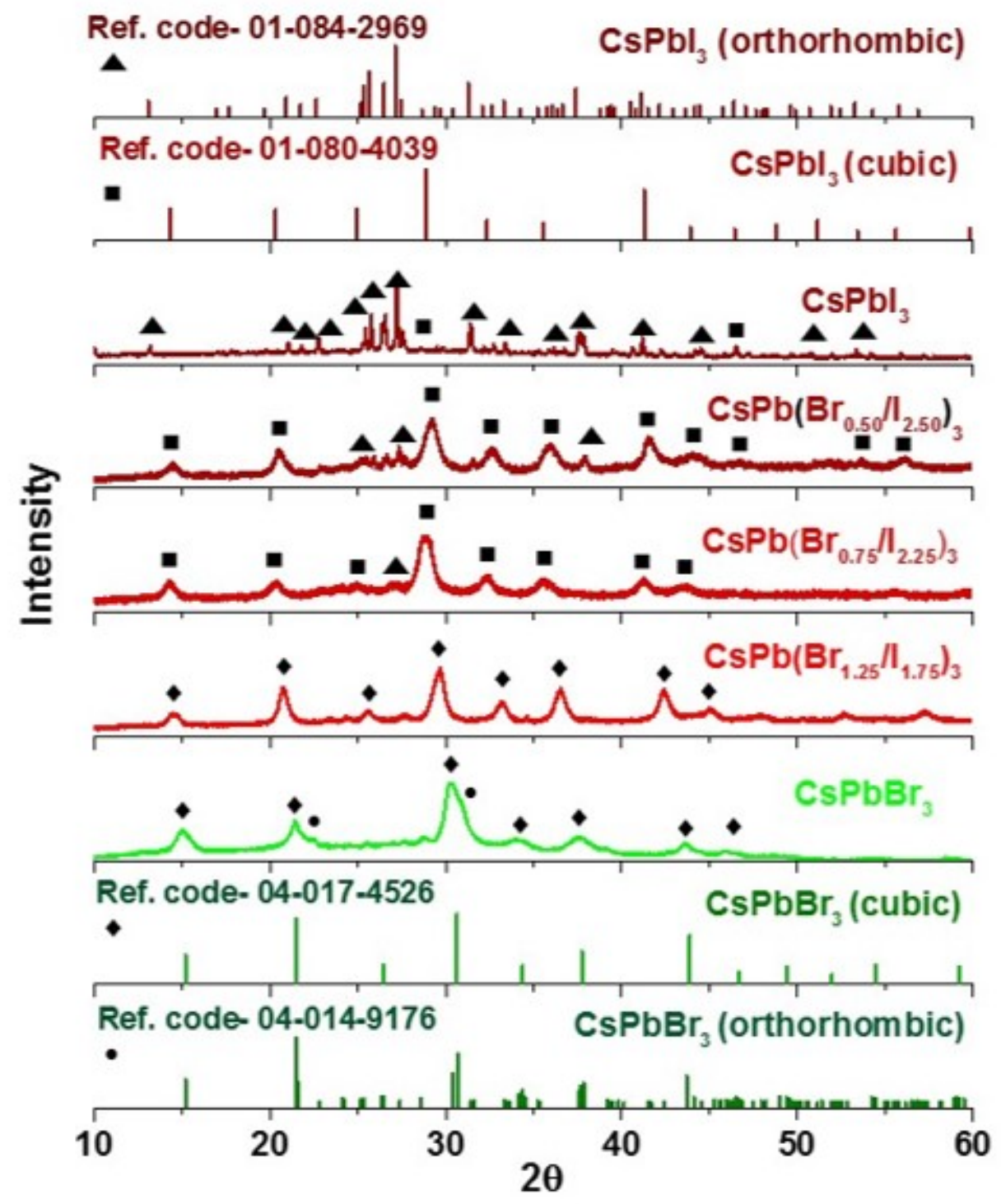

Figure 2.11: XRD spectra of CsPbX ${ }_{3}$ NCs XRD patterns were recorded by a PAN-

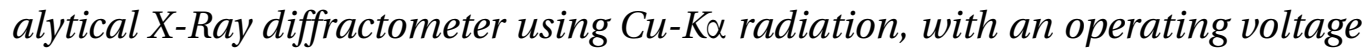
of $45 \mathrm{kV}$ and current of $40 \mathrm{~mA}$. The standard was taken from PANalytical X'Pert highscore plus. Copyright, American Chemical Society 2017. 
to the index spectra using PANalytical X'Pert HighScore Plus software data file. The change in the crystal structure from $\mathrm{CsPbBr}_{3}$ to $\mathrm{CsPbI}_{3}$ moved the two theta peak towards the lower angle. Therefore, the mixed halide NCs have peak which lies in between those of the pure $\mathrm{CsPbBr}_{3}$ and $\mathrm{CsPbI}_{3} \mathrm{NCs}$. It was observed that the $\mathrm{CsPb}_{3}$ started forming non-perosvkite orthorhombic phase, which also began to appear in iodine rich mixed halide NCs. XRD peaks of NCs were found to be broader compared to the microcrystalline peaks according to the Scherrer equation (Equation 1.39), which also confirms the formation of NCs.

\subsubsection{Optical Characterisation on Halide Composition Depen- dent Perovskite NCs}

Optical spectroscopy gives detailed study of material properties due to change in the halide composition. Each sample was diluted in hexane for the UV-Vis and PL measurements. A Cary 50 UV-Visible spectrophotometer were used to do the UV-Vis spectroscopy and a Horiba Fluorolog-3 Spectrometer were used for the PL measurement. NCs were measured in solution form and a $10 \mathrm{~mm}$ quartz cuvette with pure hexane was used to create the baseline.

All of the NCs were washed with solvent/antisolvent method as described in Section 2.2.1.2. We observed the change in the optical properties with halide composition. The PL peak was red shifted as the $\mathrm{Br} / \mathrm{I}$ ratio decreased. This is because the iodide ions are larger in size and changes the emission towards the higher wavelength. the monodispersity in the samples can be observed by FWHM of PL spectra. All of these NCs were used to fabricate perovskite LEDs, which is discussed in Chapter 3. 


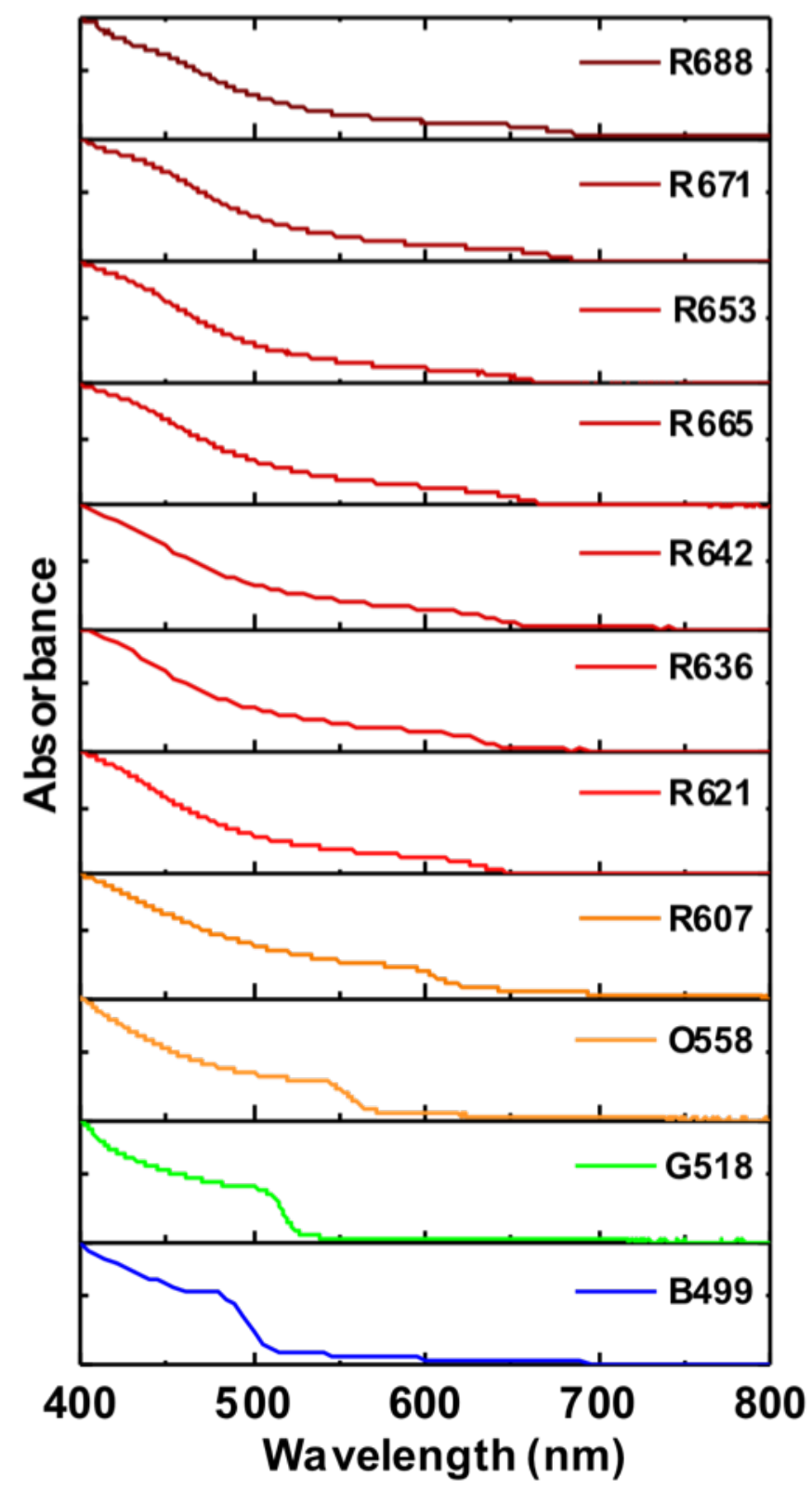

Figure 2.12: UV-Vis absorption spectra of perovskite NC samples in hexane. The absorption edge is red shifting as the $\mathrm{Br} / \mathrm{I}$ or $\mathrm{Cl} / \mathrm{Br}$ ratio is decreasing. 


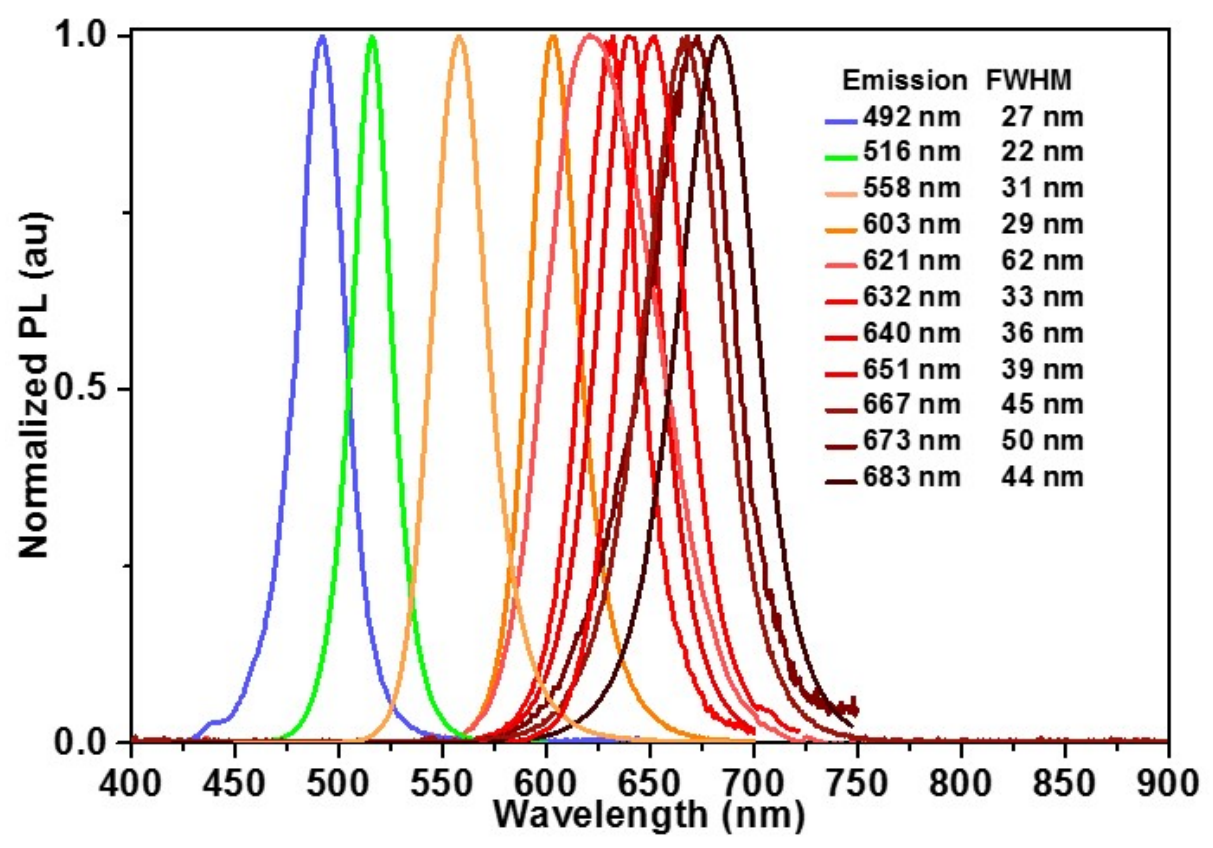

Figure 2.13: PL spectra of perovskite (pe) NCs samples in hexane. The emission peak is red shifting as the $\mathrm{Br} / \mathrm{I}$ or $\mathrm{Cl} / \mathrm{Br}$ ratio is decreasing.

\subsubsection{Characterisation on Perovskite NWs}

In order to observe the morphology and crystal structure, TEM and XRD analysis were carried out on these NWs. The TEM samples were prepared by dropcasting hexane NWs solution on a Cu-200 mesh on formvar grid. The XRD samples were prepared by drop casting the concentrated NWs solution (in hexane) on to a clean glass slide. The thickness of The $\mathrm{CsPbBr}_{3}$ and $\mathrm{CsPbI}_{3}$ were calculated to be $\approx 39$ and $14 \mathrm{~nm}$ using TEM images. However, a wide range of size distribution can be seen in the TEM micrographs. Additionally, reaction of $\mathrm{CsPbBr}_{3}$ did result in the formation of some nanoparticles along with the NWs (Figure2.14a). All the NWs were also found to agglomerate in the solution. The NWs showed very weak luminescence and the suspension in any solvent was found to be turbid. This limits the processing of these NWs for any solution processed semiconductor device applications. 


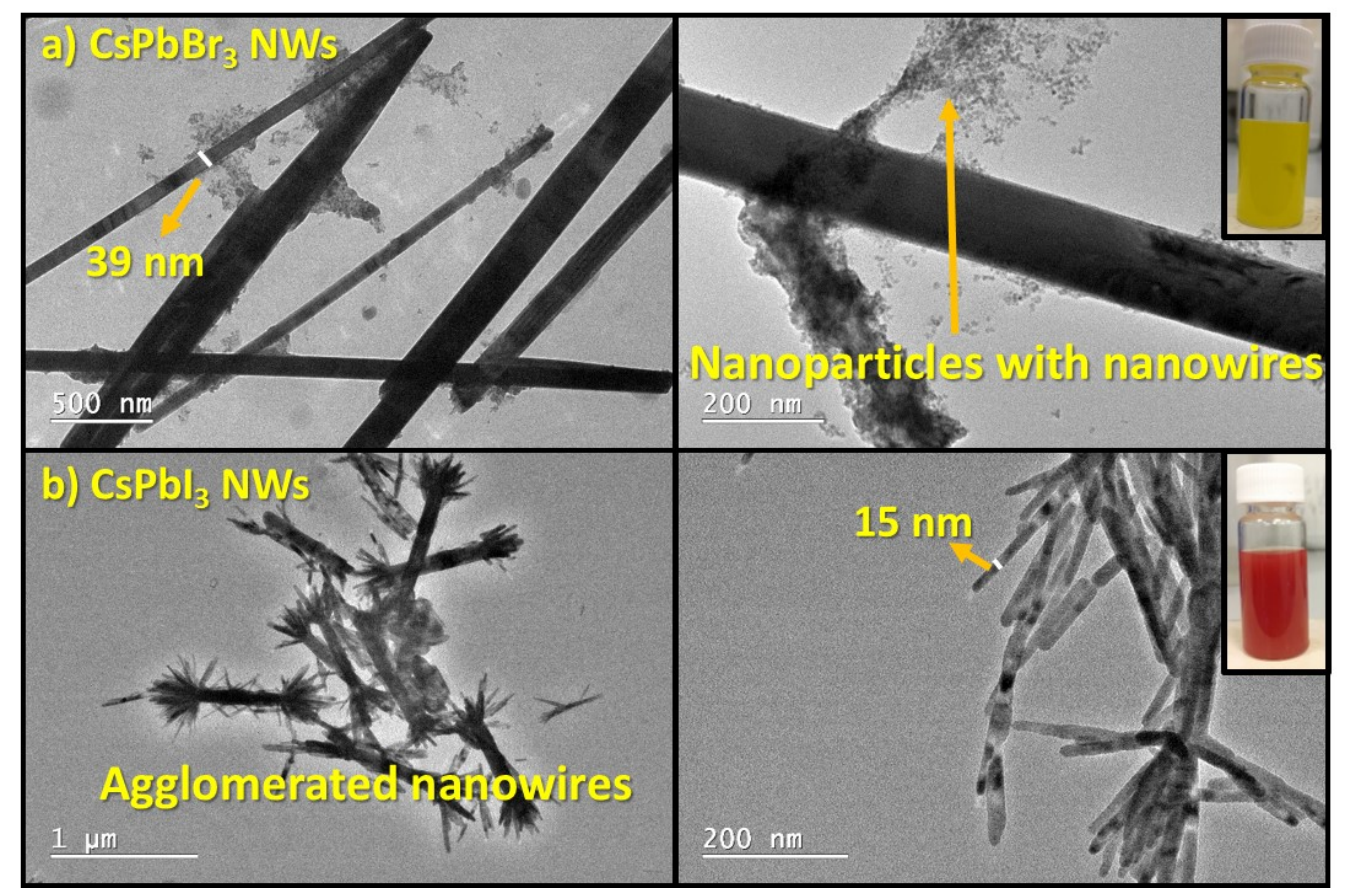

Figure 2.14: TEM micrographs of NW suspension in hexane for a) $\mathrm{CsPbBr}_{3}$ and b) $\mathrm{CsPbI}_{3}$. The photograph of each sample is overlaid on top of its TEM image. The TEM samples were prepared by drop-casting hexane NWs solution on a Cu-200 mesh on formvar grid.

The XRD spectra show the formation of $\mathrm{CsPbBr}_{3}$ and $\mathrm{CsPbI}_{3}$ perovskites. However, the NWs also exhibited the orthorhombic non-perovskite phase which is clearly visible in the XRD spectra. This orthorhombic non-perovskite phase is not suitable for optoelectronic applications. Therefore, these NWs were not found to be very efficient to be implemented in perovskite LEDs.

NWs have extraordinary electrical and optical properties but they were not found to be as efficient as perovskite NCs for the LED applications due to poor quantum yield, solubility, and size distribution [191]. Therefore, we diverted our focus only towards NCs. However, the optimisation of these NWs could be the part of future research in the Halpert Group. The application of these NCs is demonstrated as a LED in Chapter 3. 


\section{Ref. Code- 01-084-2969}
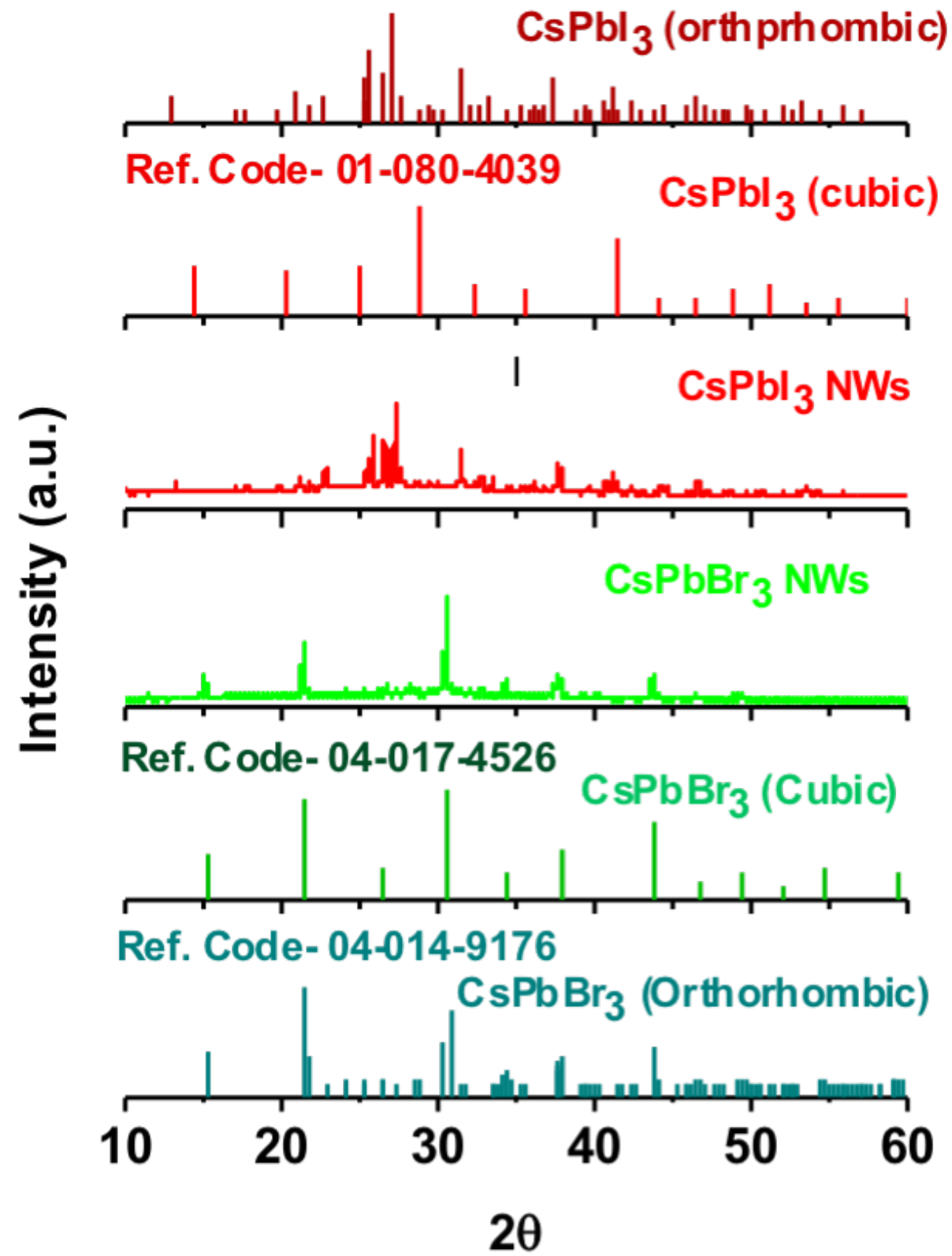

Figure 2.15: XRD spectra of perovskite NWs for a) $C s P b B r_{3}$ and b) $C s P b I_{3} . X R D$

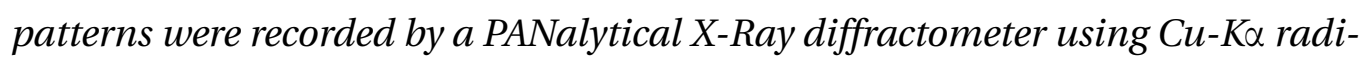
ation, with an operating voltage of $45 \mathrm{kV}$ and current of $40 \mathrm{~mA}$. The standard spectra was taken from PANalytical X'Pert HighScore Plus. 


\subsection{Conclusion}

We have synthesised size- and composition-dependent $\mathrm{CsPbX}_{3}$ NCs. A quantum confinement study was carried out on size tuned $\mathrm{CsPbB}_{3} \mathrm{NCs}$. The samples of three different sized particles were used. Steady state absorption and the PL spectra were used to confirm the confinement in these NCS. The blueshifted emission peaks with decreasing size along with the formation of high energy absorption edge in the smallest NCs showed the strong confinement effect. The increment in estimated Stokes shift with decrease in particle size also supported the similar evolution of confinement. All these studies were further supported by ultrafast transient absorption spectroscopy, which showed the multimodal GSB in the smallest NC-4.1 samples. Also, the larger NCs were found to be in weak confinement regime due to their dimension larger than the Bohr diameter $(7 \mathrm{~nm})$. Overall, the size tuneable $\mathrm{CsPbBr}_{3} \mathrm{NCs}$ were used to demonstrate the quantum confinement effect.

The NWs of these materials were also synthesised but due to their low stability and poor performance, they were not further analysed and used in optoelectronic application. However, investigating these NWs and improving their properties could be the part of future research in Halpert group. The composition dependent NCs were synthesised to be implemented in LEDs, which is discussed in next chapter. We observed the phase transition instability in pure $\mathrm{CsPbI}_{3} \mathrm{NCs}$ sample due to the softer basic nature of iodide. The monodispersity of NCs was analysed by TEM and PL spectroscopy techniques. All samples showed very high quantum yield (50-68\%) and the emission spectra was tuneable in the entire visible spectrum with very thin emission linewidth. All these properties made them very promising materials for the fabrication of LEDs. 


\section{Chapter 3}

\section{Colour Instability in $\mathrm{CsPbX}_{3}$ Perovskite Nanocrystal LEDs}

Parts of this chapter have been published in the following article:

Vashishtha P \& Halpert, JE. Field-Driven Ion Migration and colour Instability in Red-Emitting Mixed Halide Perouskite Nanocrystal Light-Emitting Diodes. Chemistry of Materials 2017.

\subsection{Introduction}

Semiconductor QDs are one of the most promising candidates for optoelectronic applications, due to their thin emission line width (FWHM), high PL quantum yield, band gap tuneability with size and shape, and relatively longer life time $[2,11,123,192]$. Over the last two decades, researchers have worked on improving the synthesis method in order to achieve monodisperesed NCs, which offers a very thin emission linewidth for various applications including LEDs [1, 13, 193]. In general, the hot-injection method offers high qual- 
ity NCs than a normal heating-up colloidal method (Section 1.2.5) [194]. Processing of NCs is also a very crucial step to wash the NCs without damaging their surface and losing the PL quantum yield. Optimising all these properties for the efficient LEDs fabrication is an area of interest in semiconductor QDs [120, 121, 195]. Various type of materials have been investigated for LEDs application, including III-V such as, GaN, InP, and AlN, I-III-VI such as $\mathrm{CuInS}_{2}$, II-VI such as GaN, and IV-VI of the periodic table, such as CdSe, CdSe/Zns, and CdS/Zns [120-122]. To date, the cadmium-based core QDs, with various Type I shell coatings, including CdS or ZnS, have been found to be the best for LEDs application due to their nearly $100 \%$ PL quantum yield, stable emission peak, precise colour tuneability with the use of confinement effect during synthesis $[1,2,4,11,123]$. The only drawback of this technology is that CdSe contains toxic cadmium, a heavy metal banned for use in electronics in some markets $[123,124]$.

Recently, $\mathrm{CsPbX}_{3}$ perovskite NCs with PL QY as high as $90 \%$ have been reported, suggesting that these could replace CdSe as the active layer in OLED structured devices (See Chapter 2) [17]. Although, $\mathrm{CsPbX}_{3}$ contains a toxic element $\mathrm{Pb}$ in it, the toxicity of $\mathrm{Pb}$ is less than $\mathrm{Cd}$ and the amount of $\mathrm{Pb}$ in $\mathrm{CsPbX}_{3}$ is less than the amount of Cd in Cd-based LEDs [125]. In perovskite structure, It is also possible to replace $\mathrm{Pb}$ with other metal such as tin or bismuth for optoelectronic applications $[22,126,127]$. However, lead- free perovskites have not been found to be very efficient yet, and further research is required $[22,196]$.

Since the reported synthesis of $\mathrm{CsPb}_{3} \mathrm{NCs}$, several groups have been successful in producing functional peNC-LEDs [21, 128-130]. Colour tuning in these materials is achieved by altering the $\mathrm{I} / \mathrm{Br}$ or $\mathrm{Br} / \mathrm{Cl}$ ratio in the crystal structure to achieve RGB emitters. However, it can also be achieved by changing the particle size up to some degree.(see Chapter 2). Song et al. were the first group to report the pure inorganic NCs based blue, green and orange peNC-LEDs with external quantum efficiencies (EQEs) of 0.07, 0.12, and $0.09 \%$, respectively [21]. Zhang et al. improved the EQE of $\mathrm{CsPbBr}_{3}$ NCs LED from 0.026 
$\%$ to $0.06 \%$ by implementing a perfluorinated ionomer with hole transport layer [130]. Other researchers worked to improve the EQE of these LEDs further and reported more efficient peNC-LEDs [129]. However, at the time of our report, they were not able to report red LEDs, which need $\mathrm{CsPb}(\mathrm{Br} / \mathrm{I})_{3} \mathrm{NCs}$ with relatively higher iodide content as an active layer of the device, due to its metastable state in cubic phase [21]. Red is a critical colour for any display, and "pure" red is generally produced from emission that peaks between 620 and $650 \mathrm{~nm}$. Lower wavelengths appear orange-red, and higher wavelengths appear less bright, because of the sensitivity of the human retina to red photons that falls off rapidly at higher wavelengths. Therefore, fabricating red LEDs using this material was a major area of interest in the research at that time. The stability of LEDs is defined as the change in performance with time, temperature, presence of moisture, and drive current. Researchers have reported that the performance of LEDs decays faster at higher temperature and drive current [197]. Perovskite materials are also extremely sensitive to moisture, which affects the performance of perovskite based LEDs [198]. Additionally, Colour stability is one of the major concern when it comes to performance of LEDs [199]. Therefore, Our main interest in this chapter was to understand and analyse the colour stability in perovskite based LEDs.

This chapter includes the fabrication of peNC-LEDs through low cost solution processing techniques. NCs were synthesised by the hot-injection colloidal method described in Chapter 2. Here, the emission is tuned in the entire visible region by changing of the halide composition. LED performance was optimised by varying the thickness of the transport and the active layers. A systematic investigation was carried out to understand the stability of these devices with changes in halide composition and applied electric field. A temperature dependent PL study was also carried out to understand the cause of colour instability in these LEDs. We also used longevity and colour stability trends that suggest next steps for improving performance in devices using these materials [20]. 


\subsection{Experimental}

This section describes the method for fabricating peNC-LEDs along with the sample characterisation techniques for absolute irradiance measurement. The techniques used for device fabrication includes spin coating, thermal evaporation, and shadow masking. The absolute irradiance graph is used to calculate the efficiency values such as, EQE (\%), luminance $\left(\mathrm{cd} / \mathrm{m}^{2}\right)$, luminous efficacy $(\mathrm{lm} / \mathrm{W})$, and current efficiency (cd/A), as described in Section 1.6.7.

\subsubsection{Fabrication of LEDs}

\subsubsection{Materials}

Poly-(ethylenedioxythiophene):polystyrenesulfonate (PEDOT:PSS) was purchased from Ossila. Poly[N,N'-bis(4-butylphenyl)-N,N'-bis-(phenyl)benzidine] (polyTPD), poly(9-vinylcarbazole) (PVK), and 1,3,5-tris(N-phenylbenzimidazol-2-yl) benzene (TPBi) were purchased from Lumtec. Acetone and isopropanol were purchased from Sigma-Aldrich. ITO coated glass substrates were purchased from Kintec. NCs were taken from the project carried out in Chapter 2.

\subsubsection{Methods}

A $12 \mathrm{~mm}$ x $12 \mathrm{~mm}$ ITO coated substrate was used to fabricate the devices. The left side of Figure 3.1a shows a schematic diagram of ITO coated glass substrate with $\mathrm{Al}$ electrodes (thermally evaporated). The device was fabricated on top of ITO coated film. Al was used as a top electrode (Figure 3.1). Each device has six pixels, each at the intersection of an Al electrode with ITO film.

ITO substrate was used as an anode. It was cleaned by ultra sonicating for 20 min in soap solution, distilled water, acetone, and isopropanol successively. 


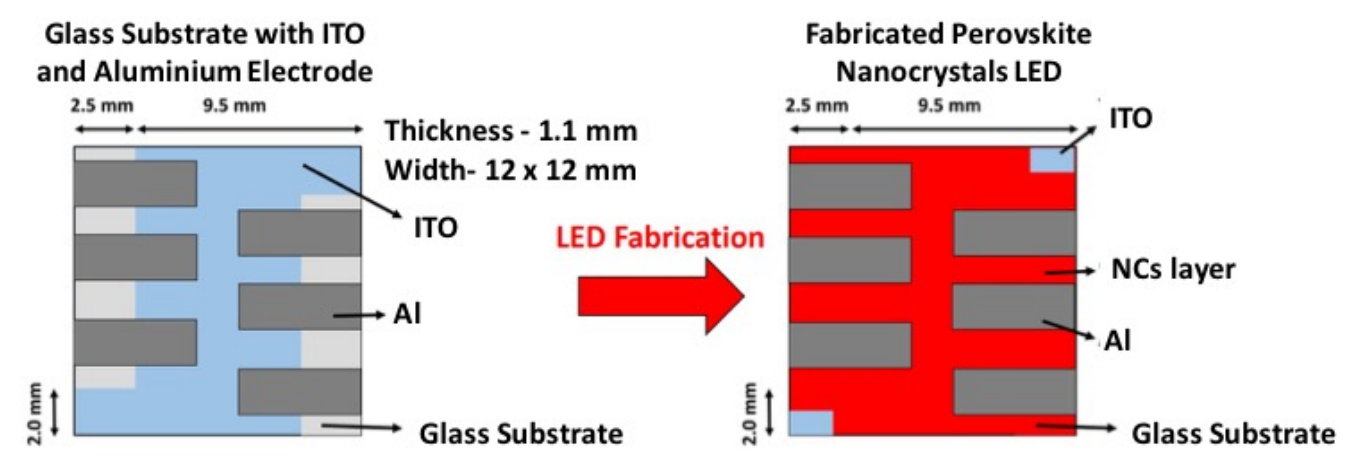

Figure 3.1: Schematic of ITO coated glass substrate with Al top electrode. Device was fabricated on top of the ITO layer and then the top electrode was thermally evaporated through a shadow mask.

They were then heated under vacuum for 30 min followed by $\mathrm{O}_{2}$ plasma cleaning for $4 \mathrm{~min}$.

Devices were fabricated using a commonly used device architecture for QDLEDs wherein, PEDOT:PSS and poly-TPD or PVK were used as a hole transport layer and TPBi were used as an electron transport layer [1]. NCs were dispersed in anhydrous hexane $(10 \mathrm{mg} / \mathrm{mL})$, and poly-TPD and PVK were dissolved in chlorobenzne (12 mg/mL). PEDOT:PSS was already dispersed in water from the supplier. PEDOT:PSS was filtered through polyvinylidene fluoride (PVDF) filters $(0.45 \mu \mathrm{m})$ and then spin-coated onto the clean ITO-coated glass substrates at $4000 \mathrm{rpm}$ for $60 \mathrm{~s}$ followed by annealing at $145^{\circ} \mathrm{C}$ for $20 \mathrm{~min}$ in air. Substrates were transferred to the nitrogen glove box. The hole transport layer, poly-TPD (12 mg/mL), was spin-coated onto the PEDOT:PSS layer at $4000 \mathrm{rpm}$ for $60 \mathrm{~s}$ and annealed at $120^{\circ} \mathrm{C}$ for $30 \mathrm{~min}$. The NCs layer was spin-coated at $2000 \mathrm{rpm}$ for $60 \mathrm{~s}$, and then devices were moved to the thermal evaporator for the deposition of a $40 \mathrm{~nm}$ electron transport layer of TPBi. Thermal evaporation was performed under high vacuum. A $100 \mathrm{~nm}$ Al electrode deposition was achieved by thermal evaporation through a shadow mask. $\mathrm{CsPb}(\mathrm{Br} / \mathrm{Cl})_{3}$ and $\mathrm{CsPbBr}_{3}$ have larger band gap, therefore PVK (12 mg/mL) was used instead of poly-TPD as a hole transport layer due to the band alignment with valence band of NCs. 


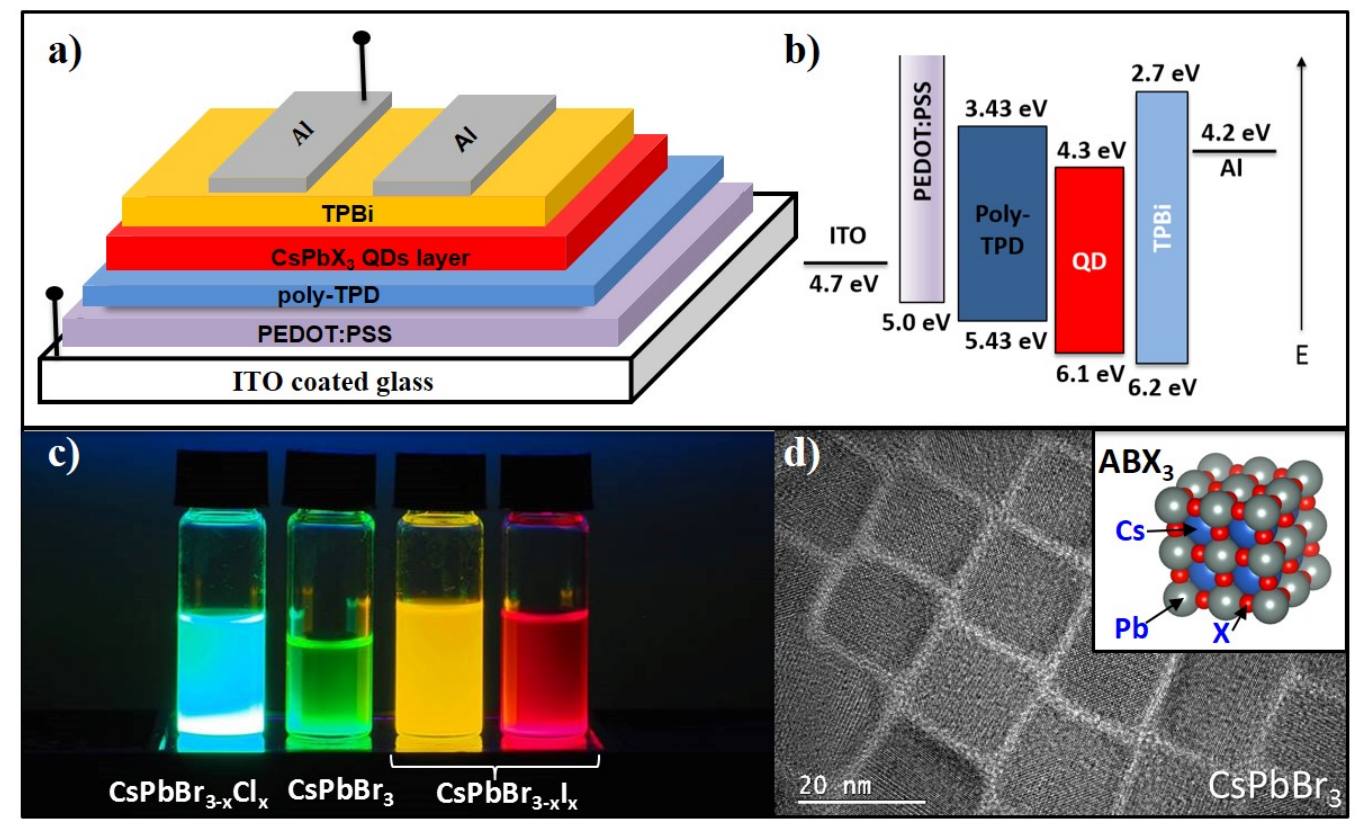

Figure 3.2: (a) Device schematic, (b) energy diagram for the peNC-LEDs investigated here along with (c) a photograph of peNCs in hexane excited by a UV lamp, and (d) a TEM of CsPbBr 3 NCs with a diagram of the perovskite NCs (inset). Energy levels relative to vacuum are shown for reference [20]. Copyright 2017 American Chemical Society.

A clear device schematic is shown in Figure 3.2 with the estimated thickness of each layer. Energy band diagram shows the energy level relative to the vacuum and the estimation of energy level was taken from the literature [130, 200, 201]. Figure 3.2c shows the photograph of perovskite NCs under $365 \mathrm{~nm}$ UV lamp. The TEM micrographs of $\mathrm{CsPBr}_{3}$ NCs are shown in Figure 3.2d. These perovskite NCs, synthesised in Chapter 2, were taken for the fabrication of peNCLEDs. 


\subsubsection{LED Measurement}

Corner of the devices was carefully scrubbed using chloroform in order to connect the probe with ITO electrode. A Keithley 236 Source Measurement Unit (SMU) instrument was used as a power source for the LED. Positive terminal of power source was connected with ITO and negative terminal was connected with $\mathrm{Al}$ electrode. The input voltage was slowly ramped up from $0 \mathrm{~V}$ and the EL spectrum was recorded at certain voltages or time intervals after the turnon voltage. EL spectra were recorded with a QE pro ocean optics spectrometer connected with an ocean optics integrating sphere (ISP-50-8-I) through an 80 $\mu \mathrm{m}$ optical fiber (QP200-2-VISNIR). Devices were placed on the port of the integrating sphere to record the EL spectra.

\subsection{Results and Discussion}

Current-voltage (IV), PL, and EL measurements were carried out to measure the device characteristics. PL measurements provided the details of the emission properties in these NC thin films. The EL along with the IV measurements were carried out to calculate the device metrics such as, external quantum efficiency (EQE), luminance $\left(\mathrm{cd} / \mathrm{m}^{2}\right)$, luminous efficacy $(\mathrm{lm} / \mathrm{W})$, and current efficiency (cd/A). Temperature dependent PL measurements were carried out on perovskite thin films to understand the effect of temperature on the device. EL at different voltages and time were recorded to study the change in the EL peak position as a function of electric field and halide composition. All of these measurements not only allowed us to measure the efficiency but also allowed us to probe the cause of colour instability in mixed halide peNC-LEDs. 


\subsubsection{Device Performance}

All devices were characterised in the ambient conditions at RT. A photograph of the peNC-LEDs at their turn on voltages (3.5-6.0 V) are shown in Figure 3.3. The EL position is labelled on top of each photograph. Blue (B499) and green (G518) LED measurements are provided for reference, while orange, orangered, and deep red devices are investigated in detail. PL spectra are taken from Figure 2.12, as the same samples were used in the fabrication of LEDs. The corresponding EL spectra were recorded at early times near the turn-on voltages. PL and EL spectral peak positions with their FWHM are labelled in the graph.

The EL peaks are similar to the PL peaks with a slight red shift of a few nanometers. This can be described to the transition from solution to thin films, where in Förster resonance energy transfer (FRET) occurs to neighbouring NCs with a lower band gap. An excited state donor molecule transfers its energy to an another ground state acceptor molecule, which is in the lower energy level, through a nonradiative process [202]. This phenomena has been observed in NC-LEDs in previous reports as well $[134,203]$. Devices investigated here are described in Table 3.1.

Table 3.1: PL and EL metrics for champion peNC devices with varying Br content.

\begin{tabular}{|c|c|c|c|c|c|c|c|c|c|c|}
\hline $\begin{array}{l}\text { LED } \\
\text { label }\end{array}$ & $\begin{array}{c}\mathbf{P L}_{\text {solution }} \\
(\mathbf{n m})\end{array}$ & $\underset{(\mathbf{n m})}{\text { FWHM PL }}$ & $\begin{array}{c}\mathbf{E L}_{\text {initial }} \\
(\mathbf{n m})\end{array}$ & $\underset{(\mathbf{n m})}{\text { FWHM EL }}$ & CIE- $x$ & CIE-y & $\mathbf{V}_{t}(\mathbf{V})$ & $\begin{array}{c}\mathbf{E Q E _ { \text { max } }} \\
\mathbf{( \% )}\end{array}$ & $\begin{array}{c}\mathbf{E L}_{\text {final }} \\
(\mathbf{n m})\end{array}$ & $\underset{(\mathbf{n m})}{\text { FWHM EI }}$ \\
\hline B499 & 492 & 27 & 499 & 28 & 0.043 & 0.491 & 6.0 & - & 500 & 20 \\
\hline G518 & 516 & 22 & 518 & 19 & 0.092 & 0.797 & 4.0 & 0.80 & 518 & 28 \\
\hline O558 & 558 & 31 & 653 & 45 & 0.711 & 0.289 & 5.0 & $1.4 \times 10^{-1}$ & 518,663 & 22,49 \\
\hline R607 & 603 & 29 & 607 & 36 & 0.632 & 0.368 & 5.0 & $2.1 \times 10^{-1}$ & 680 & 49 \\
\hline R621 & 621 & 62 & 621 & 61 & 0.654 & 0.346 & 5.8 & $6.0 \times 10^{-2}$ & 679 & 43 \\
\hline R636 & 632 & 33 & 636 & 66 & 0.664 & 0.336 & 6.2 & $7.1 \times 10^{-2}$ & 663 & 56 \\
\hline R642 & 640 & 36 & 642 & 35 & 0.708 & 0.292 & 6.0 & $2.4 \times 10^{-2}$ & 678 & 43 \\
\hline R653 & 651 & 39 & 653 & 55 & 0.698 & 0.302 & 6.0 & $1.3 \times 10^{-1}$ & 683 & 41 \\
\hline R665 & 667 & 45 & 665 & 45 & 0.720 & 0.280 & 4.0 & $2.9 \times 10^{-2}$ & 678 & 43 \\
\hline R671 & 673 & 50 & 671 & 32 & 0.728 & 0.272 & 3.9 & $<10^{-2}$ & - & - \\
\hline R688 & 683 & 41 & 688 & 35 & 0.729 & 0.271 & 3.2 & $2.7 \times 10^{-3}$ & 687 & 41 \\
\hline
\end{tabular}


As can be seen in Table 3.1, there is no EL shift in the green LEDs (G518), as the initial and final EL peak was observed to be at same position. No characteristic deep blue emission was observed from the organic molecules which were used as a hole and an electron transport layers in peNC-LEDs [1]. Pure iodide NCs $(x=3)$ were found to lose their luminescence during processing and did not produce functional LEDs. The stability of $\mathrm{CsPbI}_{3}$ is discussed in Chapter 2. The EQE was observed to be in the range of 0.02 to $0.2 \%$, which was comparable to those reported in literature value for untreated perovskite LEDs at the time this research was completed $[21,130]$. As one can see that the initial and the final EL peak position in red devices are not at the same position. All of the red devices showed red shift in the EL peak corresponding to the characteristic emission of pure $\mathrm{CsPbI}_{3}$ emission $(\approx 680 \mathrm{~nm})$. A detailed analysis of EL instability is added in the next sections. The turn-on voltage for devices fabricated in the study are given in Table 3.1. Devices with higher iodide content have lower band gap than devices with higher bromide or chloride content. It usually takes less built-in potential to turn-on the devices with lower band gap. Therefore, the devices with higher iodide content showed low turn-on voltages than rest of them. However, some lower band gap devices showed relatively higher turn-on voltages, this could be due to the instability in these devices as discussed in next sections.

Figure 3.4 shows the device metrics for peNC-LEDS at different voltages. The device statistics for all of the LEDs fabricated in this study are shown in Table 3.2. It should be noted that the NCs were capped with longer chain oleylammonium ligand, which is not ideal for the electronic applications due to its poor conductivity. Therefore, the achieved efficiency is quite reasonable. In all the devices, the EQE increased rapidly after their turn-on voltages and then it began to decrease at higher voltages. However, R688 was found to be very less efficient. In R636, the device burnt out before the scan could reach the completion. Therefore, the EL peak movement could only be observed till 7.0 V. O558 displayed the initial EL peak at $653 \mathrm{~nm}$, which was then later split into two different peaks at 518 and $663 \mathrm{~nm}$. R642-R665 LEDs, which contained moderate 


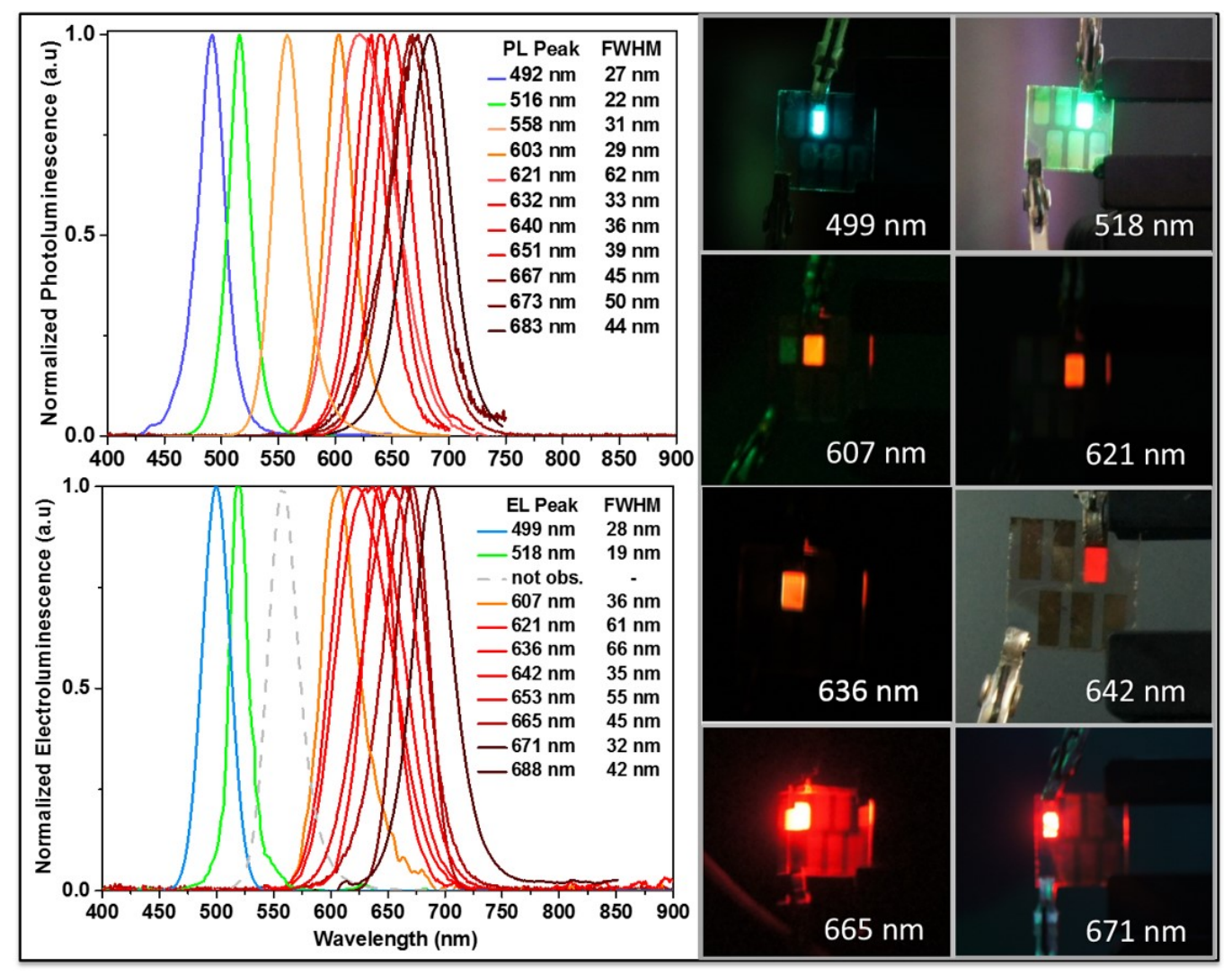

Figure 3.3: Normalised PL spectra (top) taken at $400 \mathrm{~nm}$ excitation of a solution of $\mathrm{CsPbBr} r_{3-x} I_{x}$ for varying values of $x$ from 01.3(green) to 2.75 (deep red). Blue NCs consist of $\mathrm{CsPbBr}_{3-x} \mathrm{Cl}_{x} x(x=0.75)$. Normalised EL spectra (bottom) from ITO/PEDOT:PSS/poly-TPD/peNC/TPBi/Al LEDs for these same peNCs. EL spectra were recorded immediately after turn-on (prior to red-shift). As an exception, the orange-emitting sample from the PL spectra did not produce an orange-emitting LED (gray dashes to mark the absence) as discussed in the text. At the right are shown unprocessed photos of LEDs. Copyright 2017 American Chemical Society.

amount of $\mathrm{Br}$ (see Table 3.1), were found to produce more stable LEDs with relatively high EQE and luminance.

In Figure 3.4, the luminance is found to be relatively linear as it does not depend on current or applied voltage of the device. However, the current efficiency de- 
pends on the current flow in the active layer. There is always an increase in the current as the voltage increases, therefore the cd/A was found to be relatively dynamic. EQE is also current dependent and vary similarly like current efficiency (cd/A). At the same time, luminous efficacy is nothing but the voltage dependent current efficiency, therefore, the $\mathrm{lm} / \mathrm{W}$ was found to be even dynamic than cd/A. Overall, all of these parameters were found to be changed with increase in voltage.
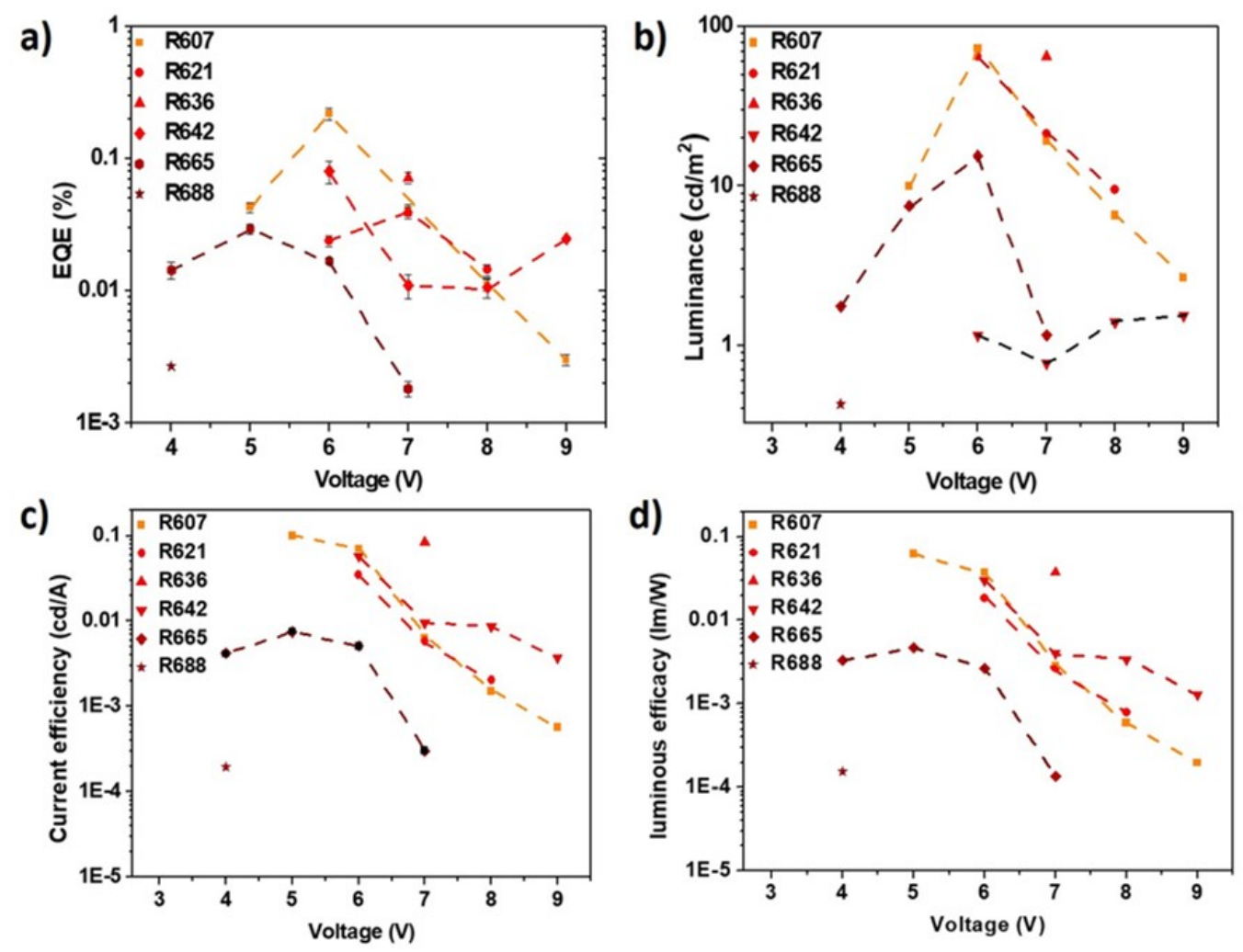

Figure 3.4: Chart of the a) EQE vs. voltage, b) Luminance vs. voltage, c) current efficiency vs. voltage, and d) luminous efficacy vs. voltage in $\mathrm{CsPb}(\mathrm{Br} / \mathrm{I})_{3} \mathrm{LEDs}$. Red devices generally show lower luminance as the peak shifts towards $680 \mathrm{~nm}$, since the luminosity scale is determined by the sensitivity of the human eye which tapers off rapidly after $650 \mathrm{~nm}$. An Ocean Optics QE Pro spectrometer was used to characterise these LEDs. Copyright, American Chemical Society 2017.

Other QD-based LEDs display a stable emission during the entire operation pe- 
Table 3.2: Device statistics on all of the fabricated LEDs at applied voltage.

\begin{tabular}{|c|c|c|c|c|c|c|c|}
\hline $\begin{array}{c}\text { LED } \\
\text { label }\end{array}$ & $\begin{array}{c}\text { Number of } \\
\text { devices }\end{array}$ & $\begin{array}{c}\mathbf{E}_{\text {initial }}(\mathbf{n m}) \\
\text { EL }\end{array}$ & $\begin{array}{c}\mathbf{E L}_{\text {final }} \\
(\mathbf{n m})\end{array}$ & $\begin{array}{c}\text { Turn-on } \\
(\mathbf{V})\end{array}$ & $\begin{array}{c}\mathbf{E Q E}_{\text {max }} \\
\mathbf{( \% )}\end{array}$ & $\begin{array}{c}\mathbf{E Q E}_{\text {average }} \\
\mathbf{( \% )}\end{array}$ & Standard deviation \\
\hline B499 & 3 & $499 \pm 1$ & $500 \pm 1$ & $6.0 \pm 0.3$ & - & - & - \\
G518 & 3 & $518 \pm 1$ & $518 \pm 1$ & $4.0 \pm 0.5$ & 0.80 & 0.67 & 0.12 \\
O558 & 3 & $653 \pm 1$ & $518 \pm 1$ & $5.0 \pm 0.4$ & $1.4 \times 10^{-1}$ & $1.1 \times 10^{-1}$ & $3.3 \times 10^{-2}$ \\
R607 & 3 & $607 \pm 2$ & $680 \pm 1$ & $5.0 \pm 0.3$ & $2.1 \times 10^{-1}$ & $1.7 \times 10^{-1}$ & $4.0 \times 10^{-2}$ \\
R621 & 3 & $621 \pm 3$ & $679 \pm 1$ & $5.8 \pm 0.2$ & $6.0 \times 10^{-2}$ & $3.69 \times 10^{-2}$ & $2.5 \times 10^{-2}$ \\
R636 & 2 & $636 \pm 1$ & $663 \pm 1$ & $6.2 \pm 0.1$ & $7.1 \times 10^{-2}$ & $3.6 \times 10^{-2}$ & $4.1 \times 10^{-2}$ \\
R642 & 5 & $642 \pm 1$ & $678 \pm 2$ & $6.2 \pm 0.1$ & $2.4 \times 10^{-2}$ & $1.7 \times 10^{-2}$ & $6.2 \times 10^{-3}$ \\
R653 & 5 & $653 \pm 2$ & $683 \pm 2$ & $6.0 \pm 0.1$ & $1.3 \times 10^{-2}$ & $9.0 \times 10^{-3}$ & $2.7 \times 10^{-3}$ \\
R665 & 6 & $665 \pm 2$ & $678 \pm 4$ & $4.0 \pm 0.2$ & $2.9 \times 10^{-2}$ & $2.5 \times 10^{-2}$ & $4.5 \times 10^{-3}$ \\
R671 & 1 & 673 & - & 3.9 & $<10^{-2}$ & - & - \\
R688 & 2 & 688 & 687 & 3.2 & $2.7 \times 10^{-3}$ & $3.1 \times 10^{-3}$ & $5.7 \times 10^{-4}$ \\
\hline
\end{tabular}

riod. For instance, CdSe QDs samples with comparable quantum yields have been used to produce LEDs with $>1 \% \mathrm{EQE}$ using this device architecture and display no shift in the EL peak even after prolonged use at a high current density $\left(10 \mathrm{~mA} / \mathrm{cm}^{2}\right)[1]$. However, these mixed halide peNC-LEDs were found to be red shifted under operation condition, which cause the colour instability in the LEDs. They also showed relatively low EQE than CdSe QD LEDs [1]. A detailed study was carried out to understand this phenomena.

\subsubsection{EL Peak Shifting in Mixed Halide Red LEDs}

All mixed halide devices showed significant shift in the initial EL peak position. Table 3.1 shows the starting and final EL peaks for each device during operation from $0 \mathrm{~V}$ to the maximal voltage for each device (at 8-10 V) prior to burnout. As is apparent from the table, the EL peaks of the red-emitting peNC-LEDs (devices R607-R665) shift significantly to higher wavelengths as the voltage increases. An EL measurement as a function of time was carried out to analyse 
the reversibility of peak shift in detail. NCs emitting at $635 \mathrm{~nm}$ was prepared for this LED fabrication (Figure 3.5a) and the device was constructed as described in the methods section. The PL in this device was observed to be at $638 \mathrm{~nm}$. The device was operated at $6.0 \mathrm{~V}$ for several min to record the EL at different time interval. Figure 3.5c shows the EL spectra of the same device. The EL peak moves from its initial position of $641 \mathrm{~nm}$ to $684 \mathrm{~nm}$ in $13 \mathrm{~min}$ of operation period. To evaluate the reversibility of the PL peak shift, PL measurements were carried out after certain time intervals of device operation. It was found that the peak shift was not immediately reversible. However, after several hours (16 h), the PL peak did return to its initial position (Figure 3.5d). The similar peak shift and reversibility were observed in all of the mixed halide red LEDs. The initial EL peak moved with the increase in voltage or time towards the higher wavelength to values between 670 and $680 \mathrm{~nm}$ (Figure 3.6), which is near the characteristic emission peak of pure $\mathrm{CsPbI}_{3} \mathrm{NCs}$ [17]. This limits the value of halide composition tuning in these materials and represents a significant challenge to the development of mixed halide perovskite LEDs. In summary, the peak shift was observed in all the red LEDs with the PL peak reversibility after several hours.

These data allow us to discount several possible factors causing the emission peak shift. One possible reason for peak shifting could be due to Stark effect, which is the shifting or splitting of spectral lines of molecule in the presence of external electric field. However, it is clear from Figure 3.5 and 3.6 that the band shift is too extreme to be due to a Stark shift or a similar electrostatic change in the band energies of individual NCs [204]. Emission from organics or interfacial exciton would be clearly distinguishable from NC emission by the width of emission peak, as the organic molecules used in these LEDs have higher FWHM and peak positions at lower wavelength $(400-500 \mathrm{~nm})[1,205,206]$. The other possible reason for the red shift could be the growth of NCs in the thin film during device operation, a phenomena which has been seen in peNCs films [207]. The growth of NCs could be caused by three possible events: 
a)

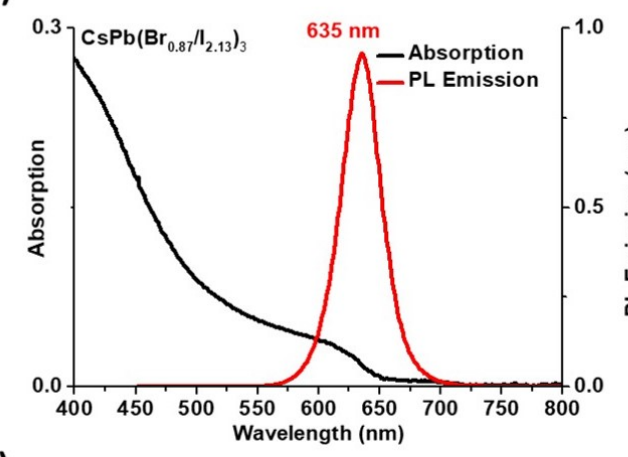

c)

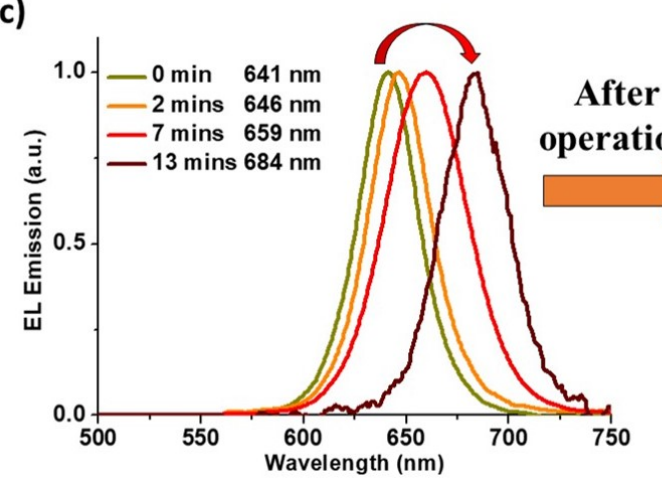

b)

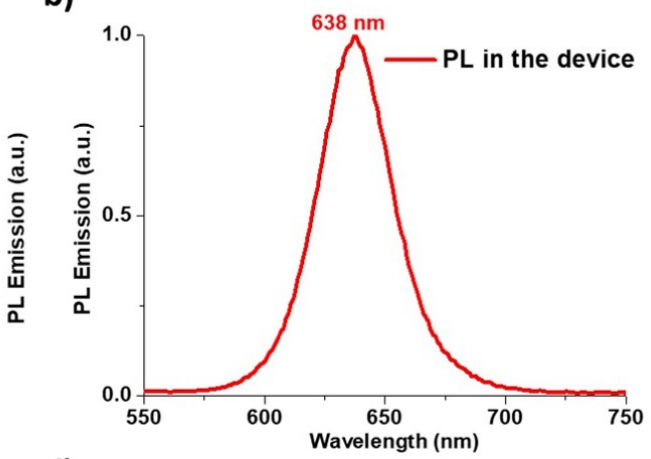

d)

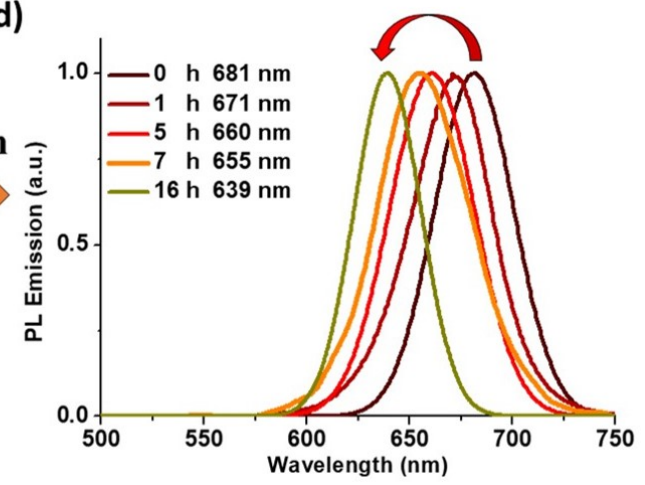

Figure 3.5: a) UV-Vis and PL of NC-635 in hexane exciting at $400 \mathrm{~nm}$, b) PL of the $N C-635$ in the LED thin film exciting at $400 \mathrm{~nm}, \mathrm{c})$ EL of the LED device using NC635 on 6.0 V at varying times, and d) PL of the NC-635 LED after the operation at varying times after shutdown. The EL peak shifts from its initial value towards $\approx 680 \mathrm{~nm}$. However, after $\approx 16 \mathrm{~h}$ the PL emission shows the reversibility towards its initial position. Copyright American Chemical Society 2017.

1. Incorporation of unreacted precursors present in improperly purified NC solutions and deposited in the thin film.

2. Ostwald ripening in which large NCs could go at the expense of their neighbour particles, due to the chemical activity of the ligands.

3. Fusion of neighbouring NCs, an effect has been previously observed in perovskite NCs (Section 2.3).[208]

The confinement effect can only tune the emission spectra by $\pm 15 \mathrm{~nm}$, which is explained in Section 2.3.3. The peNCs used in these LEDs have a particle size 
of $15 \mathrm{~nm}$ (Figure 2.9) which is larger than the Bohr diameter $(7 \mathrm{~nm})$. Therefore, these NCs should fall in the weak confinement regime (Figure 2.7), which further limits the emission shift towards higher wavelengths [188]. The observed emission shift in NCs is more than $50 \mathrm{~nm}$ which can never be due to the growth of NCs [209]. In addition, fusion or growth of the NCs should be an irreversible process. Whereas, we have observed a reversibility of emission peak after the device operation (Figure 3.5). The last possibility could be the movement of the ions within the NCs and that the composition of individual emitters within the NC film changes during the operation. This means, the bromide and iodide ions are possibly moving within the NCs, and creating iodide rich domains which emit in the higher wavelength. This ion migration could occur due to the several possible reasons including heating in the device during operation, electric field, or excitation density. Therefore, a temperature and electric field dependent study is needed to probe this phenomena. In the next section the effect of temperature on the optical properties of perovskite NC is explained.

a)

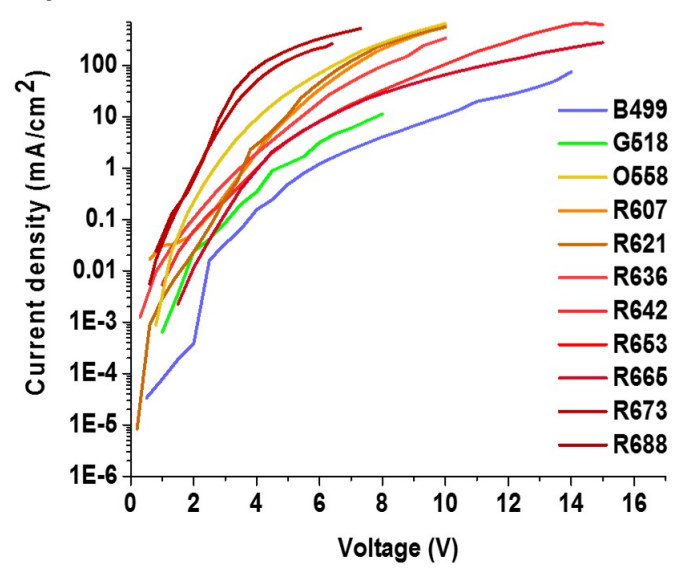

b)

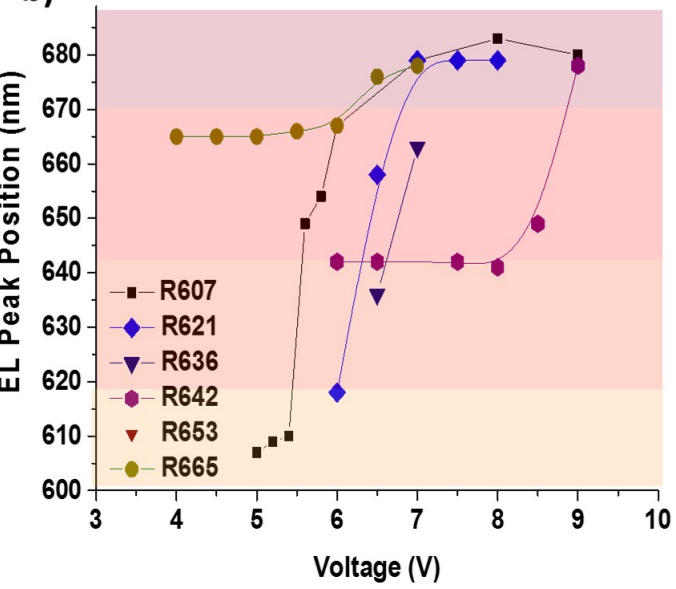

Figure 3.6: a) J-V curve of peNC-LEDs, showing higher current density for the less stable NCs sample and b) Plot of EL peak positions vs voltage for mixed halide red peNC-LEDs (607-665). 

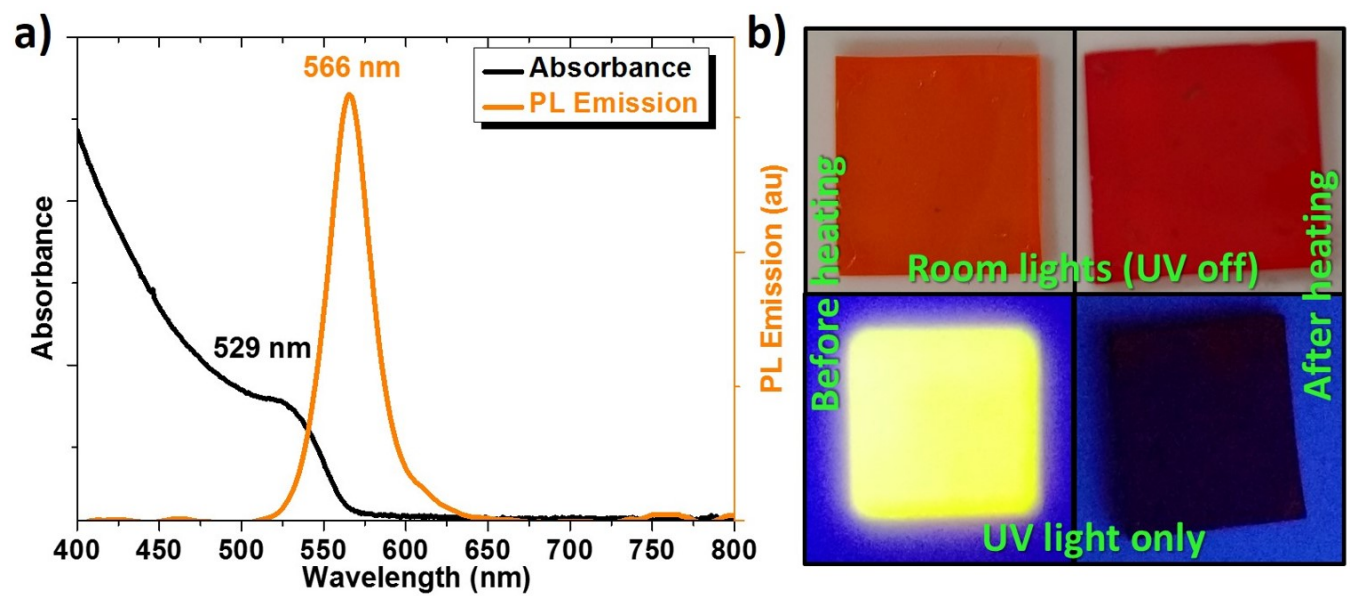

Figure 3.7: a) UV-Vis and PL of peNCs in hexane, exciting at $400 \mathrm{~nm}$, shows the emission at $566 \mathrm{~nm}$ with first absorption edge at $529 \mathrm{~nm}, \mathrm{~b}$ ) photo of NC-566 thin film before and after the heat treatment under room light with UV lamp off (top) and UV light only (bottom).

\subsubsection{Temperature Dependent PL Study on Mixed Halide NCs}

When an electric field is applied to a LED, there is a subsequent increase in temperature up to to a certain degree due to the power dissipation in the diode [210]. To observe the change in PL spectra while the film is heated, a temperature dependent PL measurement was carried out. Where the film was heated up to certain temperature and the PL was recorded constantly using QE Pro Ocean Optics spectrometer connected with a $80 \mu \mathrm{m}$ optical fiber (QP200-2VISNIR). Figure 3.7a shows the absorption and PL spectra of mixed halide peNCs with bromide to iodide loading molar ratio of $0.63: 0.37$. Due to the mixed halide structures, the PL emission was observed at $566 \mathrm{~nm}$ with $33 \mathrm{~nm}$ FWHM (Figure 3.7a), which is somewhere in between the emission from pure $\mathrm{CsPbBr}_{3}$ and pure $\mathrm{CsPbI}_{3} \mathrm{NCs}$ (Chapter 2). The band gap was calculated to be $2.3 \mathrm{eV}$ from the first absorption edge in Figure 3.7a.

These NCs were dissolved in hexane $(40 \mathrm{mg} / \mathrm{mL})$ then spin cast onto a cleaned glass slide in a $\mathrm{N}_{2}$ glove box. This spin coating process was repeated 6-8 times 

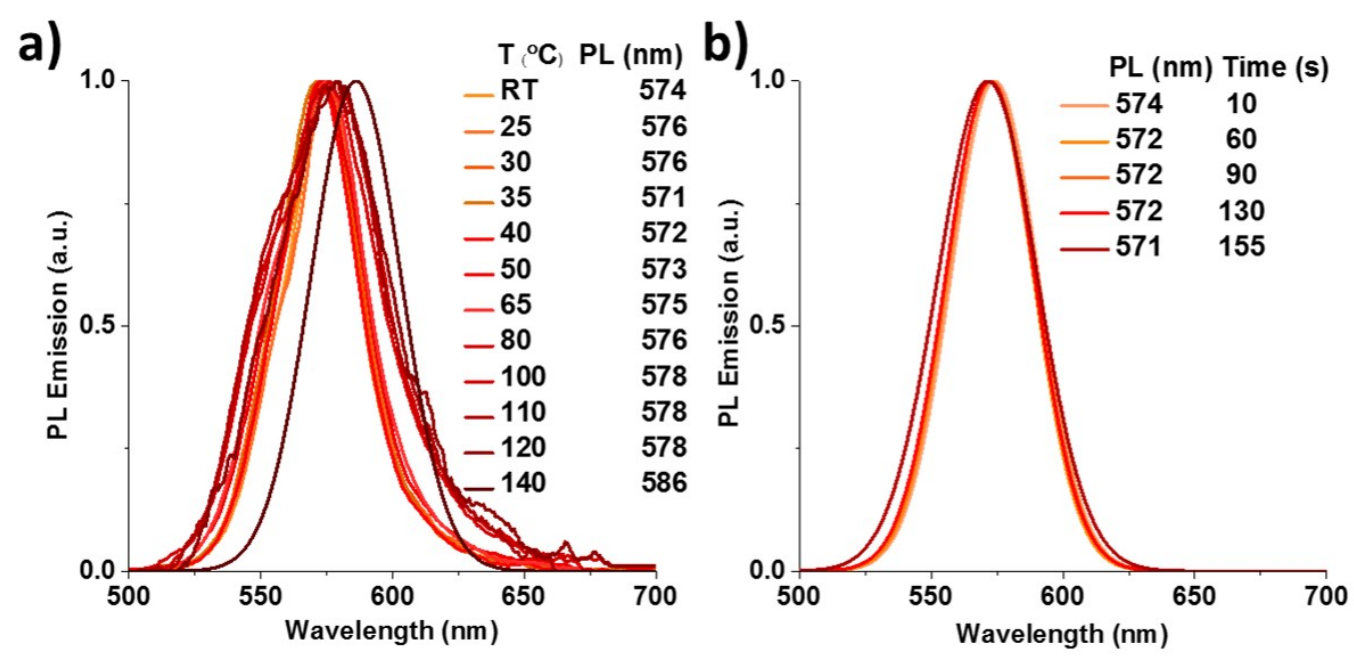

Figure 3.8: a) PL measurement of NC-566 film at varying temperature from room temperature to $140{ }^{\circ} \mathrm{C}$. and b) PL measurement when the NC-566 film approaches $75^{\circ} \mathrm{C}$. Copyright American Chemical Society 2017.

to form thick, close-packed films (Figure 3.7b). The sample was then placed on a hot plate and temperature was slowly raised from $25^{\circ} \mathrm{C}$ to $140{ }^{\circ} \mathrm{C}$. At room temperature, NCs films were highly luminescent (Figure 3.7b). However, after heating up to $140{ }^{\circ} \mathrm{C}$, the NCs lost all of their emission, which can be seen in Figure 3.7b. The PL was recorded using an Ocean Optic QE pro spectrometer while the NCs film was excited using a $365 \mathrm{~nm}$ UV lamp. Figure 3.8a shows the PL spectra of NCs films. Initially, the PL peak was recorded at $574 \mathrm{~nm}$, which is slightly red shifted than the NCs solution, due to the transition from solution to film. As the temperature increased, the PL peak moved slightly around the same position (Figure 3.8a). However, at higher temperature $\left(>100^{\circ} \mathrm{C}\right)$, a slight red shift of $10 \mathrm{~nm}$ was observed just before the NCs lost their emission. The shift near the melting point of NCs is possibly due to the fusion of the NCs, an effect that has been observed previously in perovskite materials (Chapter 2) $[181,208]$.

To observe any emission peak splitting due to the temperature gradient in the perovskite films, another PL measurement was conducted, where the tempera- 
ture was set at $75^{\circ} \mathrm{C}$ and PL was recorded as the substrate warmed quickly from room temperature to $75^{\circ} \mathrm{C}$. During this time, there should be initially a significant temperature gradient in the film as the film was about $1 \mu \mathrm{m}$ thick and the heat was supplied from the bottom of the substrate. Again, no peak splitting or significant PL shift was observed here at any point in the heating cycle (Figure 3.8b). These results suggest that the peak shifting observed in peNC-LEDs is not solely a function of temperature. While heat does aid generally in ionic mobility, ionic separation must be possible due to an electric field. Therefore, a systemic study of emission shift with change in voltage was needed, which is described in the next section.

\subsubsection{Halide Composition and Voltage dependent EL Studies}

In this study, all red devices were tested at different voltages. Specially, device R665 and R607 were tested for field stability by slowly scanning from the turnon voltage $(\approx 4-5 \mathrm{~V})$ to $9.0 \mathrm{~V}$ at a rate of $5 \mathrm{mV} \mathrm{s}^{-1}$. As illustrated in Figure

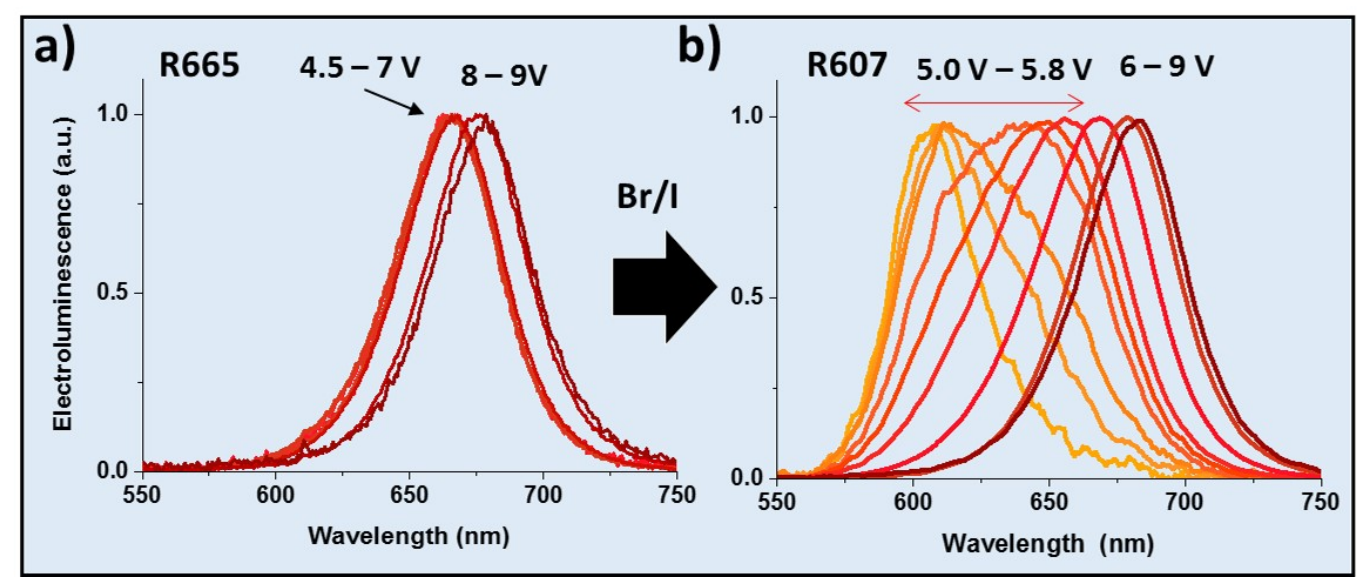

Figure 3.9: Peak-normalised EL spectra for example device (a) R665 and (b) R607 showing spectral shapes and red-shifting of the peak during J-V measurement from near their respective sample PL peak to $\approx 680 \mathrm{~nm}$. The effect is noticeably stronger for the high-bromide content peNC-LEDs. 
3.9, devices with NC films with a high Br/I (R607) ratio shifted earlier and at field strengths lower than those with films with a high I/Br (R665) ratio. R665 showed the stable EL till 7.0 V and the EL appeared to move from $8.0 \mathrm{~V}$. Whereas, In R607, the EL peak started moving just after the turn-on voltage. The thickness of the organic and NC layers was similar, presumably the field strength at the interface is similar for each LED. Deeper red devices also appear to be more stable in that the onset of the peak shift occurs at a higher applied bias. This indicates that a high iodide content in fact produces more stable peNC-LEDs and that high-iodide NCs appear to be more resistant to field-induced separation. A similar behaviour was observed in all the devices (Figure 3.10). Device R642 was found to be more stable at higher electric field than R621. Device R688 was stable at its original position. However, the efficiency of this device was found to be very low due to the instability of $\mathrm{CsPbI}_{3} \mathrm{NCs}$. The higher rate of ion migration in bromide rich material is also consistent with the theoretical reports, where the ionic migration barrier in perovskites is found to be lower for bromide $(0.23 \mathrm{eV})$ than for iodide $(0.29-0.30 \mathrm{eV})$ [211]. Thus, a huge halide composition dependency was found in those mixed halide red device against electric field. However, at the end, all the devices showed the EL peak around $680 \mathrm{~nm}$. This colour instability is the result of ion migration in mixed halide NCs. The NCs are losing some bromide ions and creating iodide rich species, which is emitting at higher wavelength. The increase in the voltage possibly led to an increase in the rate of ion migration, which created higher iodide content emitters. This resulted in the final emission at $\approx 680 \mathrm{~nm}$.

On the other side, peak shift has given us the indication of field dependency in ion migration. Therefore, the same devices were operated at different constant voltage for certain time intervals to observe the change in EL (Figure 3.11). Similar halide composition dependency was observed in this measurement. At constant voltage, the EL in R607 was found to be shifted at early time compare to the EL in R665. As observed in R665, it took 12 min for the EL peak to reach $678 \mathrm{~nm}$ at $4.5 \mathrm{~V}$ but it only took $45 \mathrm{~s}$ for the EL peak to reach $680 \mathrm{~nm}$. A similar behaviour was seen in R607, at $5.1 \mathrm{~V}$, it took about $65 \mathrm{~s}$ for the peak to move 
completely till $680 \mathrm{~nm}$. However, at $6.5 \mathrm{~V}$, the peaks moved to $679 \mathrm{~nm}$ in only about $15 \mathrm{~s}$. When the device R607 ran at $9 \mathrm{~V}$ the initial EL peak was recorded at $678 \mathrm{~nm}$. This shows that at higher voltage the peak moved quickly and with further increase in voltage, the peak shift was so fast that the initial EL was observed at $676 \mathrm{~nm}$. Therefore in both LEDs, the EL peak shift was found to be faster at higher voltages. To have a clear overview of peak shifts over time at constant voltages, a graph between peak wavelength vs time was plotted in Figure 3.12. At low voltages, the linearity is disturbed by a relatively slower initial

a) R621

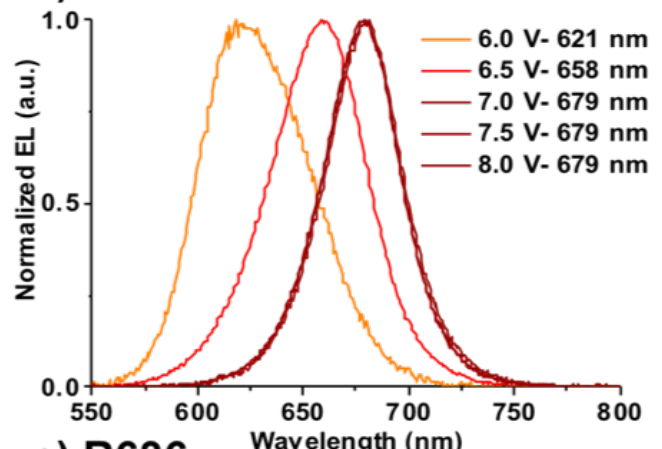

c) $\mathbf{R 6 3 6}$

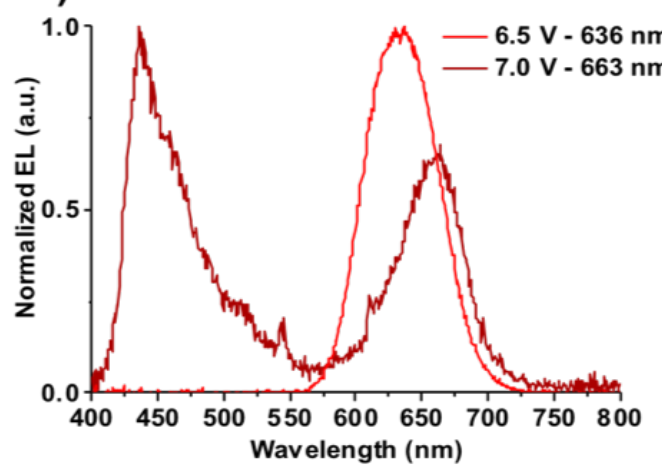

\section{b) R642}

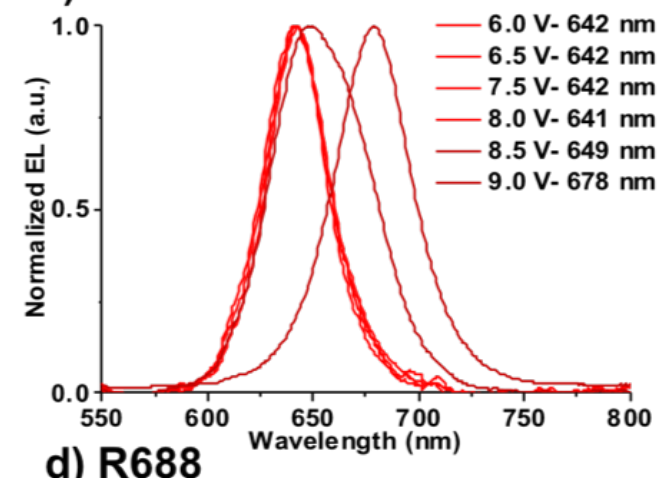

d) $\mathbf{R 6 8 8}$

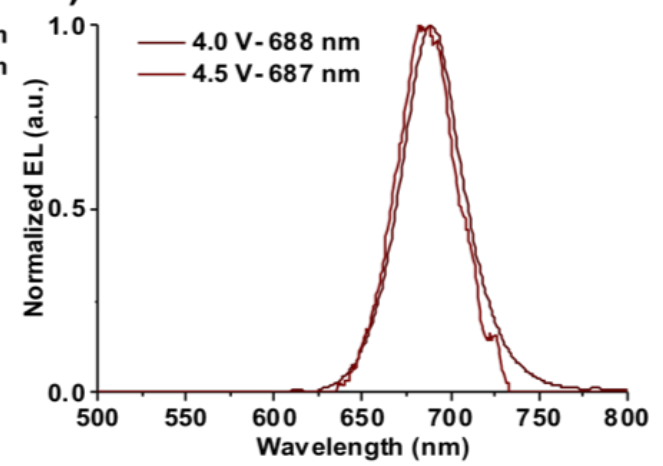

Figure 3.10: EL spectra with respect to voltage of device a) R621, b) R642, c) R636, d) R688. Similar behaviour can be seen here, the EL peak moves from its initial position to the higher wavelength $(\approx 680 \mathrm{~nm})$ as the voltage increases. Device with higher bromide content found to be less stable against the electric field. Device R636 burnt out before scan could reach its completion. Copyright American Chemical Society 2017. 
a) R665

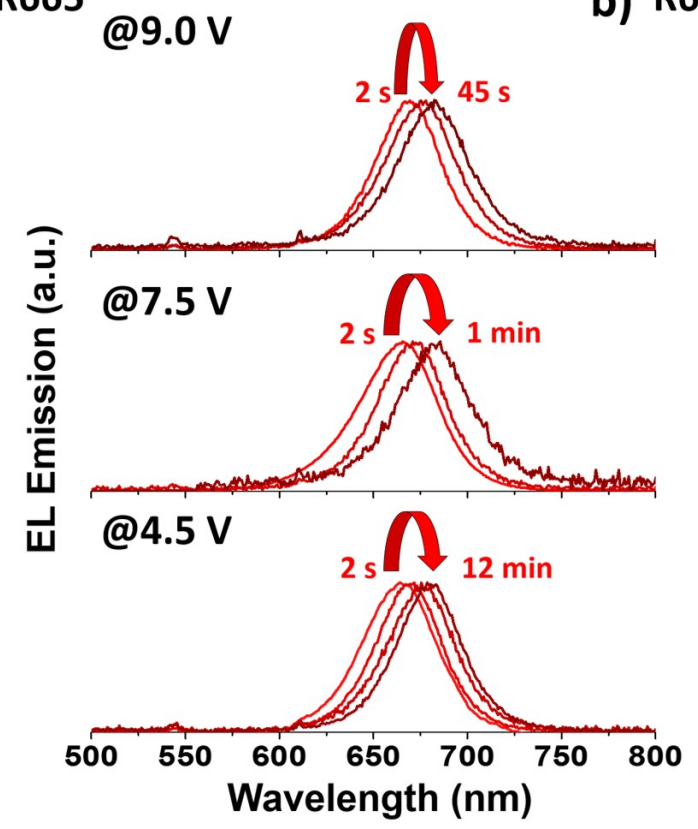

b) R607

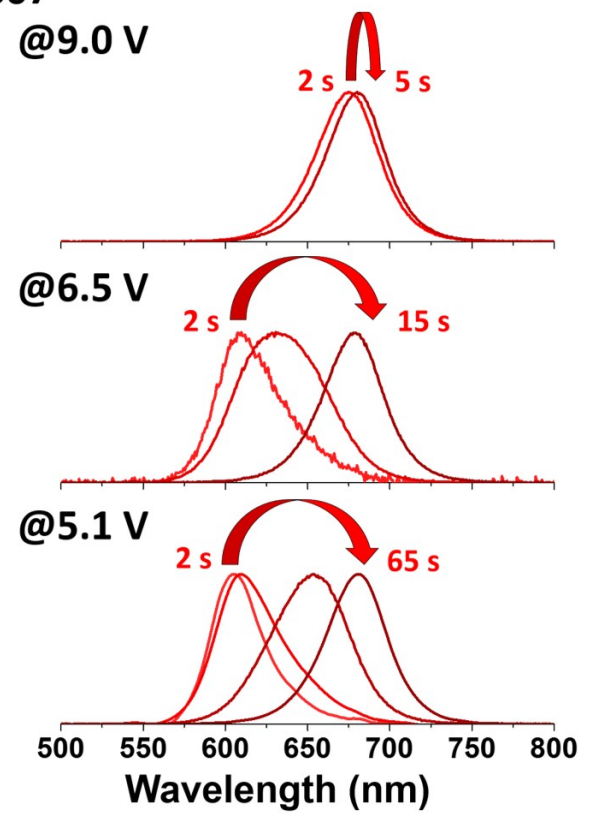

Figure 3.11: EL peaks movement at different constant voltages with respect to time in a) R607 and b) R665. The EL peak shift is faster at higher voltage and in R607, which contained higher bromide content. Copyright American Chemical Society 2017.

increase. At low power (low voltage, where current increases with the voltage), devices take some time reach their steady state temperature, after which the rate of change appears voltage dependent. Another graph was plotted in Figure 3.13 to observe the relationship between the rate of peak shifts with voltage, current, and power.

The inverse of the final time $\left(t_{F}\right)$ in Figure 3.13, should be equal to the average rate of peak movement, which itself can be viewed as a reasonable proxy for the rate at which ions are migrating within the NC film. As can be seen from the plot of voltage vs $1 / t_{F}$, the rate varies linearly with the voltage, nonlinearly with the current density, and nonlinearly with the power for R607. If the migration effect is dependent on the local field strength, this could be expected to vary linearly with the voltage at applied voltages above the threshold voltage for the 

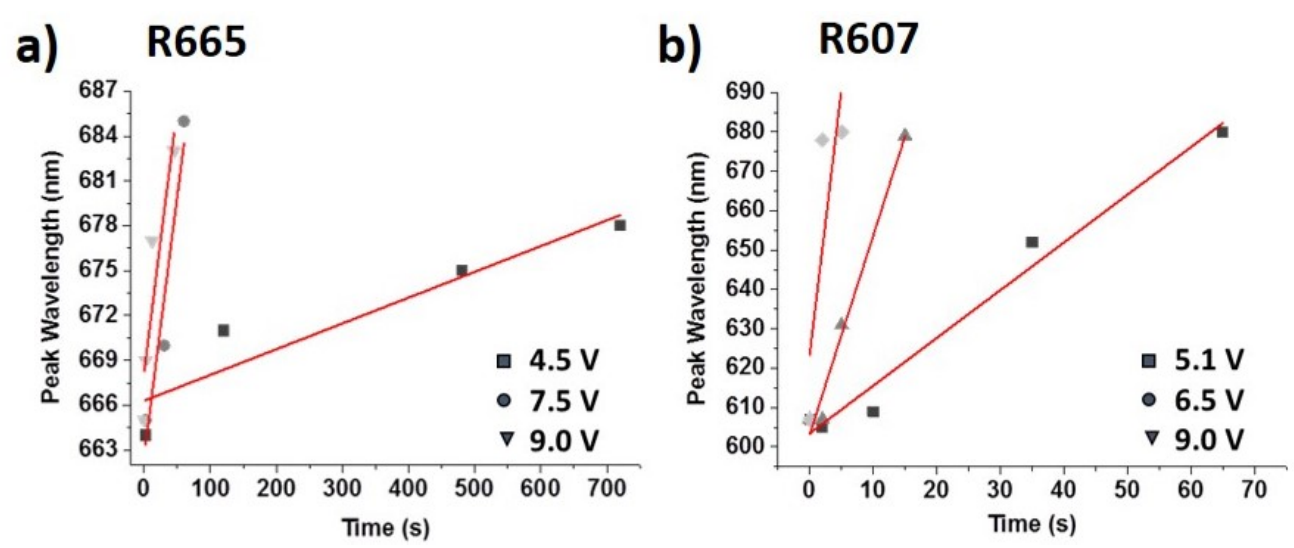

Figure 3.12: A chart of peak wavelength vs time for devices R607 and R665. Linear trend lines are added to show a general trend of peak movement.

a)

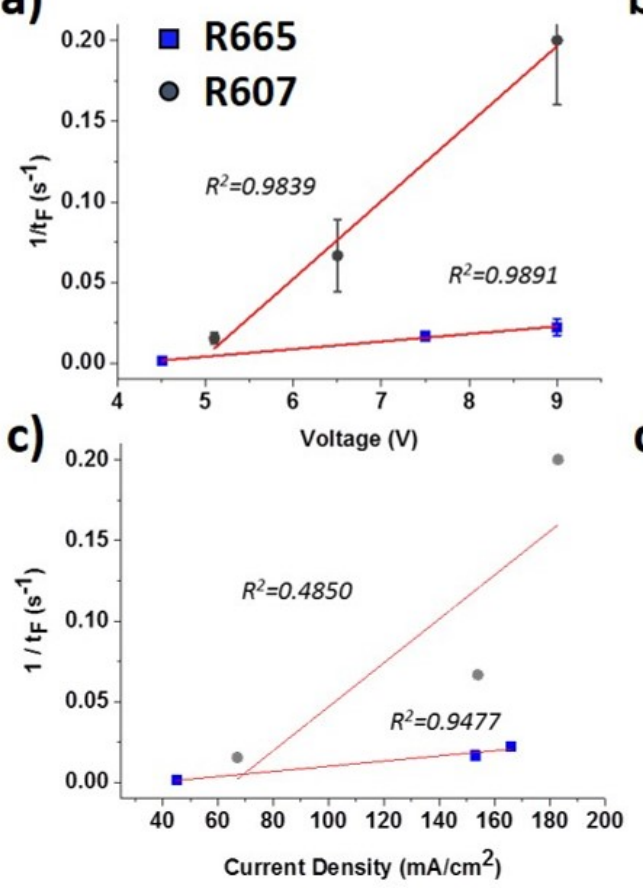

b)

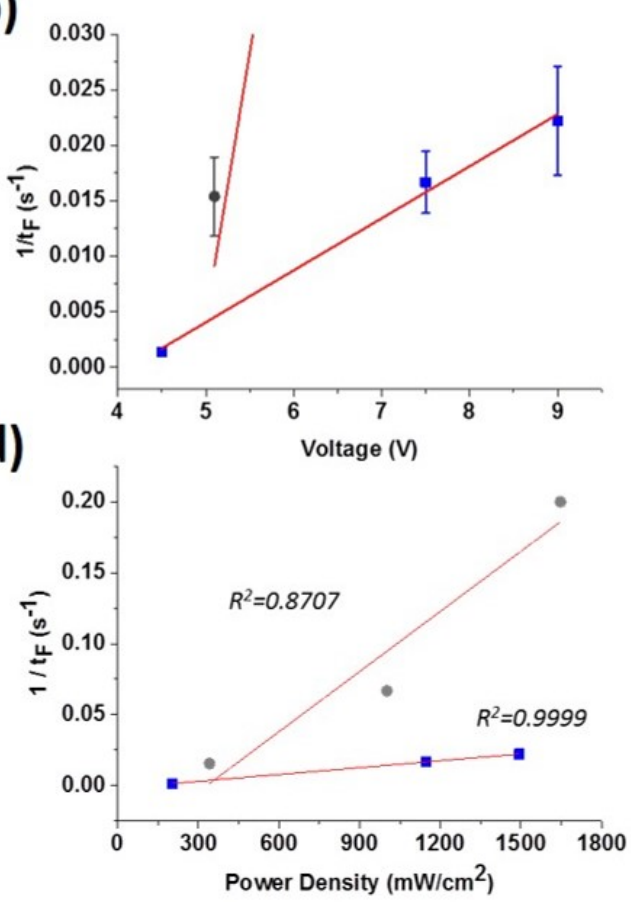

Figure 3.13: a), b) Plots of the applied voltage, c) current density, and d) power density, versus the inverse of the final time $\left(t_{F}\right)$ at which the curve has reached its end state at $\approx 680 \mathrm{~nm}$ and has stopped shifting. Copyright American Chemical Society 2017. 
device. Similarly, if the rate was solely dependent on the temperature, it could be expected to be linear with respect to power, where $\mathrm{dT} / \mathrm{dt}$ is proportional to P, such that $\mathrm{T}(\mathrm{t})$ is proportional to P.t, in the early stages of heating (before cooling effects become significant and the temperature reaches a steady state). It should be noted that the current density depends on the voltage by a power law, $\mathrm{V}_{n}$ where $\mathrm{n}$ is approximately 2 to 4 . Electrical power is the product of voltage and current so $\mathrm{P}$ should vary by equation 3.1. In a simple device model, electric field varies with voltage by equation 3.2 , where $d$ is the distance between two relatively infinite rectangular electrodes, $\mathrm{E}$ is the magnitude of the electric field and V is the voltage applied to those electrodes. This data suggests that ion separation is indeed field driven, because it is linear with voltage [20]. However, there is some indication that heating is required to start the process (Figure 3.12) but an applied field must be present to induce separation in this system, and indeed, the rate of separation appears to be highly voltage dependent.

$$
\begin{gathered}
V . V^{n}=V^{n+1} \\
V=E . d
\end{gathered}
$$

Device function appeared to be highly dependent on the voltage as the rate of peak shift was found to be linear with the applied voltage. However, the real dynamics within the device may be significantly more complex. Band bending and charge injection will affect the internal electric field at the interface, as will the differences and changes in the local dielectric in the NCs, the ligands and the surrounding organic semiconductors. We were only able to probe at macro level and a more realistic model may be warranted to fully understand the ion separation phenomenon within these devices. Additionally, the the extent of halide composition dependence was yet to be clearly understood. Therefore, NCs with even higher bromide content (63\%) was synthesised for the fabrication of LEDs and the instability in this device is investigated in the next section. 


\subsubsection{Colour Instability in High Bromide Content LED (0558)}

The peNCs with a very high bromide content, which were used in the unstable orange-emitting peNCs in device O558, gave completely unstable emission. The peak shift of the emission in these devices provided a strong indication of the source of the colour instability of mixed Br/I peNC-LEDs. Using this device, we were able to examine the source of emission from NCs composition more clearly. The PL of the peNCs in solution prior to thin film formation was found to be $558 \mathrm{~nm}$ (red line in Figure 3.14a), corresponding to yellow-orange emission. The PL of the thin film was observed at $552 \mathrm{~nm}$ (red line in Figure 3.14a), due to absorption and re-emission slightly skewing the initial PL measurement in the concentrated solution to higher wavelength [20]. The device was then operated at $5.0 \mathrm{~V}$ and the first EL emission was found to be at $\approx 650 \mathrm{~nm}$. This indicates that the red shift was too fast to be observed at its original position. This behaviour was not observed in other devices where the bromide content was relatively lower (R607-R688). That means incorporation of bromide led to highly unstable LEDs.

The same device was operated for longer time at $7.0 \mathrm{~V}$, and the initial peak was split into two different peaks, one red shifted to $665 \mathrm{~nm}$ with a FWHM of 50 $\mathrm{nm}$ and the other peak at $518 \mathrm{~nm}$ with a FWHM of $22 \mathrm{~nm}$. The photograph of LED also looks the mixture of green and orange colour (Figure 3.14). The peak splitting was never observed in any mixed halide peNC-LEDs before. As the device was run for longer period of time at the same voltage (7.0V), the intensity of the red peak slowly decreased and eventually disappeared with the increase in the intensity of green peak. Eventually, the LED was found to be emitting pure green colour at $518 \mathrm{~nm}$. The $518 \mathrm{~nm}$ peak and thin emission line width are unmistakably characteristic of large $\mathrm{CsPbBr}_{3}$ NCs (pure bromide) with bulklike band edge emission (i.e., in the weak confinement regime) [188, 212]. The other peak at $\approx 665 \mathrm{~nm}$ matches the emission of peNCs with a $0.50 / 2.50 \mathrm{Br} / \mathrm{I}$ ratio similar to those used in R665 LEDs. Thus, the mixed halide composition was split into two different compositions, iodide rich and pure bromide emit- 

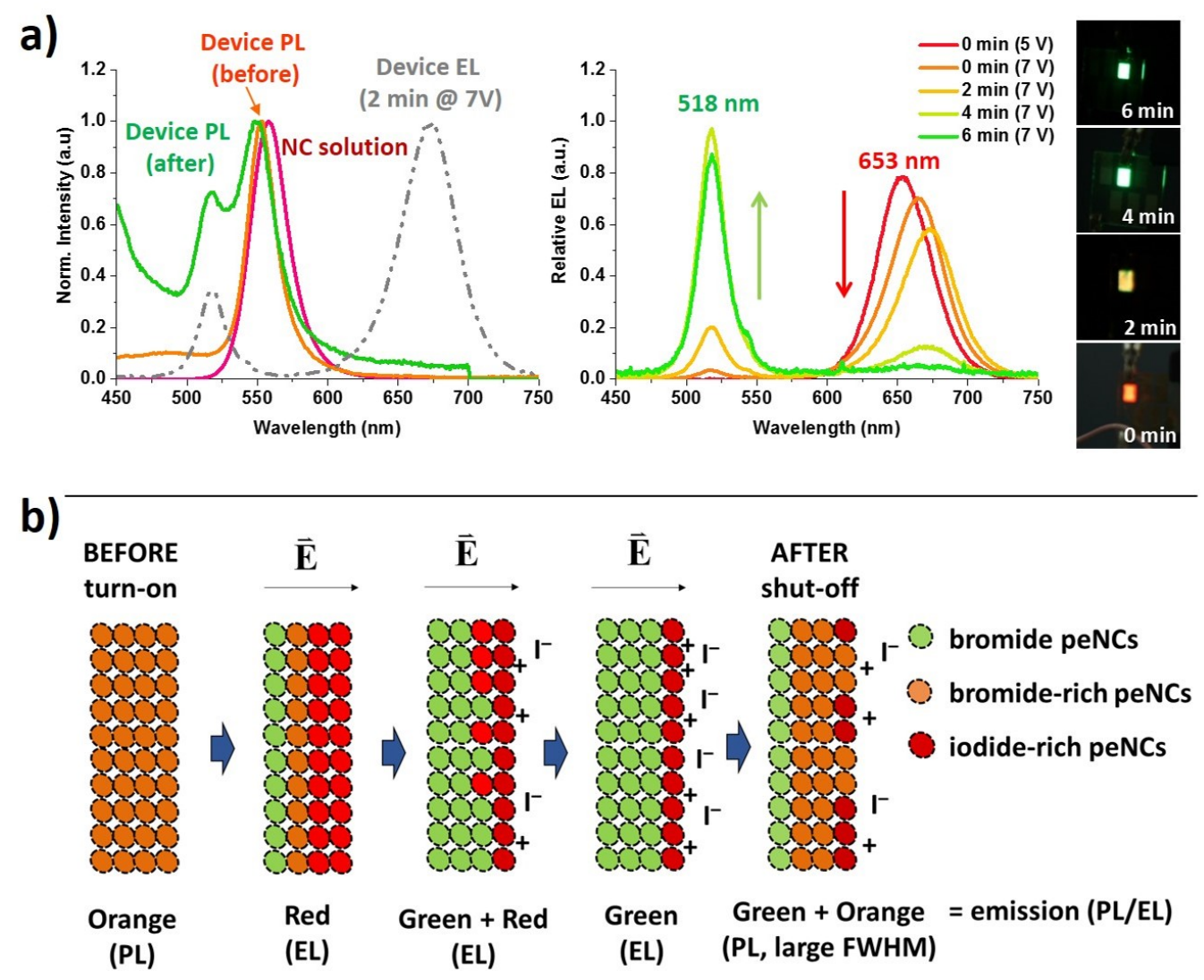

Figure 3.14: PL spectrum (left chart) of a solution prior to thin film formation (red line) and of the LED before (orange line) and after (green line) device operation. The EL from the device after operation at $7.0 \mathrm{~V}$ for $2 \mathrm{~min}$ is included for reference (dashed gray line). After the initial PL measurements, the voltage for the LED was raised to the turn-on voltage of $6.2 \mathrm{~V}$ until EL was observed and then switched to $7.0 \mathrm{~V}$ to ensure observation of ionic separation. EL spectra (top center chart) and photos (top right) of the same LED were taken at the turn-on voltage and at $7.0 \mathrm{~V}$ and then at 2 min intervals until the spectrum had shifted completely. b) A diagram of ion migration creating green-and red-emitting NCs, as well as charging of red NCs at later times. Copyright American Chemical Society 2017. 
ters, with eventual disappearance of iodide rich (red) emitters.

The eventual disappearance of the red emitters is particularly astonishing, because Förster resonance energy transfer (FRET) between NCs should give a high probability of excited NCs transferring energy to the lowest-band gap NC within $\approx 1 \mathrm{~nm}$ (FRET radius) [195]. That means the NCs emitting at $518 \mathrm{~nm}$ should transfer their energy to the NCs with redder emission in the excited state. However, no emission peak was observed in higher wavelength after a few min. That indicates that either the green-emitting NCs are not within $1 \mathrm{~nm}$ of the red-emitting NCs or the red-emitting NCs are incapable of emitting due to one of the following reasons:

1. Chemical alteration of the red NCs, which makes them incapable to emit.

2. The presence of nonradiative recombination pathways in red NCs due to an increased level of Auger recombination as these NC becomes charged during the operation [213]. In Auger recombination, the electron in excited state transfer their energy to an another electron in conduction band, to increase the energy of second electron. Then, the first electron recombines with the hole in valence band (See Section 1.4.4).

3. Phase change to the larger band gap non-perovskite orthorhombic phase in higher iodide content red NCs. As $\mathrm{CsPbI}_{3}$ structure is relatively unstable and preferably forms a non-perovskite orthorhombic phase. (Section 2.3.3) $[214,215]$.

In either case, the red NCs were found to be incapable of emitting light. Figure $3.14 \mathrm{~b}$ shows the mechanism behind this phenomena. Initially, in the device, there are few monolayers of $\mathrm{CsPb}\left(\mathrm{Br}_{1.88} / \mathrm{I}_{1.12}\right)_{3} \mathrm{NCs}$, which shows the $\mathrm{PL}$ at 552 $\mathrm{nm}$. When the electric field was applied, these mixed halide NCs lost some $\mathrm{Br}$ and gained some I, which made them iodide rich NCs. At the same time, some of the NCs, lost iodide and became bromide rich NCs. At $5.0 \mathrm{~V}$, only iodide rich NCs were able to emit emission at $\approx 650 \mathrm{~nm}$. But, the increase in electric field resulted in the increase in green emitter monolayers. This produces 
higher number of purified domains of $\mathrm{CsPbBr}_{3} \mathrm{NCs}$ within the thin film, which are then electrically excited via charge injection, or FRET of excitons from the surrounding organic layers, and emitting at $518 \mathrm{~nm}$ [216]. Ion separation here creates a quantity of iodide free NCs large enough that many of these are unable to FRET transfer energy to their now absent red-emitting neighbors. Instead, excited states formed on green-emitting NCs can radiatively relax with the characteristic green emission of a large diameter (>10 nm) $\mathrm{CsPbBr}_{3} \mathrm{NC}$. Thus, the iodide rich NCs lost their emission and only the emission from $\mathrm{CsPbBr}_{3}$ could be observed.

The green emission peak is apparent only in device 0558 because of the very large quantity of bromide present in the initial sample. In devices with an iodide content greater than that of $\mathrm{O} 558$ (e.g., R607-R688), there may be greenemitting NCs, but never enough for green emission to be observed. Instead, their excited states were transferred via FRET to neighboring deep red emitters, or peNCs with deep red domains, in the films that emit uniformly near 670-680 $\mathrm{nm}$ once the maximal degree of ion separation has occurred [20].

Further evidence of internanocrystal ionic separation was apparent when the PL of the thin film was measured again, and the initial emission peak near 550 $\mathrm{nm}$ was observed again (Figure 3.14a). Once the field is off, the ions are free to re-equilibrate in the lowest free energy state, comprising of mixed halide ions. Therefore, they should have moved back to their original PL position which was at $552 \mathrm{~nm}$. However, the remaining green emission peak in the "after" PL shows that the degree of ion movement in these particles was so extreme that some NCs were irreversibly changed to pure $\mathrm{CsPbBr}_{3} \mathrm{NCs}$ and were unable to complete the ion exchange to a homogeneous system (Figure 3.14a). The redistribution of ions between NCs was consistent with exchange in perovskite NCs in solution [214]. Indeed, the irreversibility of the emission shift in NCs containing a very high level of bromide is more prevalent than the "after" PL would suggest, because again FRET in these films skews the film emission towards red emitters. The wide FWHM of the two peaks in the "after" PL is an indication 
that ion re-exchange is proceeding because the thin emission line widths of the two species present during operation have widened as individual NCs begin to exhibit a wider distribution of $\mathrm{Br} / \mathrm{I}$ ratios during the re-exchange process once the device is turned off. Thus, adding more bromide led to a completely unstable LEDs, which showed peak splitting and extreme peak shift in the EL.

\subsubsection{Stability Test on Mixed Halide LEDs}

In addition to the efficiency, the stability of a semiconductor device is very important. Therefore, a stability test was conducted on a mixed halide peNC-LED. All mixed halide LEDs were found to be red shifted. However, LEDs with moderate amount of bromide and iodide content (R642-R665) produced relatively stable emission and higher EQE. Therefore, a device with optimised amount
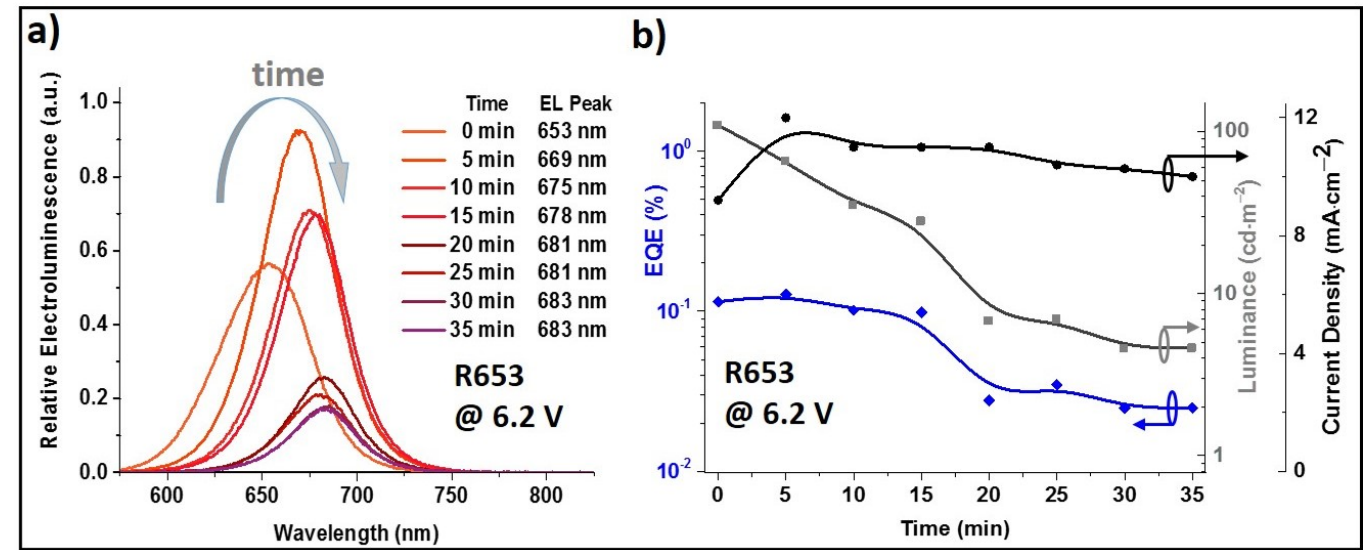

Figure 3.15: (a) Chart of the relative intensity of the EL spectra for R653 over a period of 35 min at a bias of $6.2 \mathrm{~V}$ and $\approx 120 \mathrm{~mA} \mathrm{~cm}^{-2}$ and (b) chart of the EQE, luminance, and current density evolution during a 35 min operation. The current density and EQE were stable within $\pm 15 \%$ of the average over the first 15 min. By contrast, the luminance (apparent brightness) decays as the peak shifts to the red from 653 to $683 \mathrm{~nm}$, where the human eye is less efficient. This demonstrates that long-lived devices are feasible but highly dependent on the colour stability of the NC. Copyright American Chemical Society 2017. 
of bromide and halide was constructed for the stability test, in which a constant voltage was applied for a particular amount of time. The EL peak moved from its initial position to the $\approx 680 \mathrm{~nm}$ as the electric field was applied (Figure 3.15a). This phenomena was observed in all the mixed halide LEDs emitting in the red spectral region. However, the unpackaged device was run over $35 \mathrm{~min}$ in ambient conditions, considering the fact that organic transport layers (polyTPD, TPBi, and PEDOT:PSS) are air and moisture sensitive. The device showed the maximum EQE of $0.13 \%$. In addition, the EQE remained above $75 \%$ of the maximal value during a $15 \mathrm{~min}$ continuous operation at $6.2 \mathrm{~V}$ and $200 \mathrm{~mA} / \mathrm{cm}^{2}$ of current density, decaying to $170 \mathrm{~mA} / \mathrm{cm}^{2}$ during a $35 \mathrm{~min}$ operation in air. Therefore, the lifetime result suggests that long-lived peNc-LEDs could be produced if perovskite NCs were compositionally stable or if the ion migration in the active layer could be suppressed. This ion migration in perovskites is currently a widely research topic. The synthesis of composition stable NCs could be the part of future research in the Halpert Group.

\subsection{Conclusion}

We have constructed peNC-LEDs with emission in the entire visible region by solution processing technique. More importantly, for the first time, we focused in the red emission spectral region to show the precise colour tuning from 600$680 \mathrm{~nm}$. In red devices, by analysing the the EL peak evolution with respect to the halide composition of the as-synthesised peNCs, we were able to discern that differential ionic separation is driving colour instability in these devices. This explains the observable red-shifting of the EL peak with respect to the PL peak of the constituent peNC samples. This also explains observations of EL peak splitting where two separate NC samples were apparently produced by applying a bias voltage over time. From these data, it appears that iodide rich

devices were significantly more stable under high fields and that an increasing bromide content, to tune the band edge toward the desirable emission range 
of 620-640 nm, seems to decrease the device stability. We have also shown that the major cause of colour instability is the electric field as the average rate of peak movement was found to be linear with voltage.

Ultimately, if peNC composition instability can be controlled, the colour instability and related inefficiency may not be an insurmountable challenge. Ionic separation, to form separately emitting NC domains in NC films (as opposed to thin films), has not previously been observed and indicates that there are further dynamics in NCs systems that may be worth exploring in future. With improvements in surface coating chemistry, these results suggest that peNC-LEDs could achieve device metrics that are more competitive with those of commercial NC-LED technologies.

So far, we have observed the remarkable properties such as, high PL quantum yield, composition tuneability, and thin emission line widths with tuneable colour in perovskite NCs. However, the EQE in these devices were found to be very low. This could be due to fast ion migration, bulky ligands, large exciton binding energies, or surface defects [86]. One of the most promising approaches to overcome this issue is to use a quasi $2 \mathrm{D} / 3 \mathrm{D}$ perovskite structure. The processing technique for this device is even easier than peNC-LEDs. Additionally, a quasi-2D perovskite combines the colour purity and tuneability of QDs with the conduction properties of 3D crystals [17, 35, 36]. Chapter 4 describes the fabrication of quasi-2D perovskite LEDs in detail. 


\section{Chapter 4}

\section{Quasi-2D Inorganic Metal Halide Perovskite LEDs}

\subsection{Introduction}

Inorganic metal halide perovskites are one of the most promising materials for LEDs due to their composition colour tuneability, high PL quantum yield, and low trap density [21, 132, 217, 218]. As observed in Chapter 3, perovskite NCs exhibited narrow emission with high PL quantum yield (QY). But, to date, the LEDs based on perovskite NCs always suffered with low EQE and stability, due to their bulky organic ligands and surface defects [20,21, 86]. Ligand exchange is one of the possible way to overcome the surface defects. However, even with ligand exchange, these LEDs have been performed poorly due cracks and poor morphology in NC films [27]. It was also found that if the NCs are very closely packed, they tend to quench them self which results in low PL quantum yield [26]. Therefore, relatively larger ligands are required to have high QY in NCs. On the other hand, 3D micorocrystalline perovskite showed relatively broader emission, high quantum yield only at high excitation density, low sta- 
a)
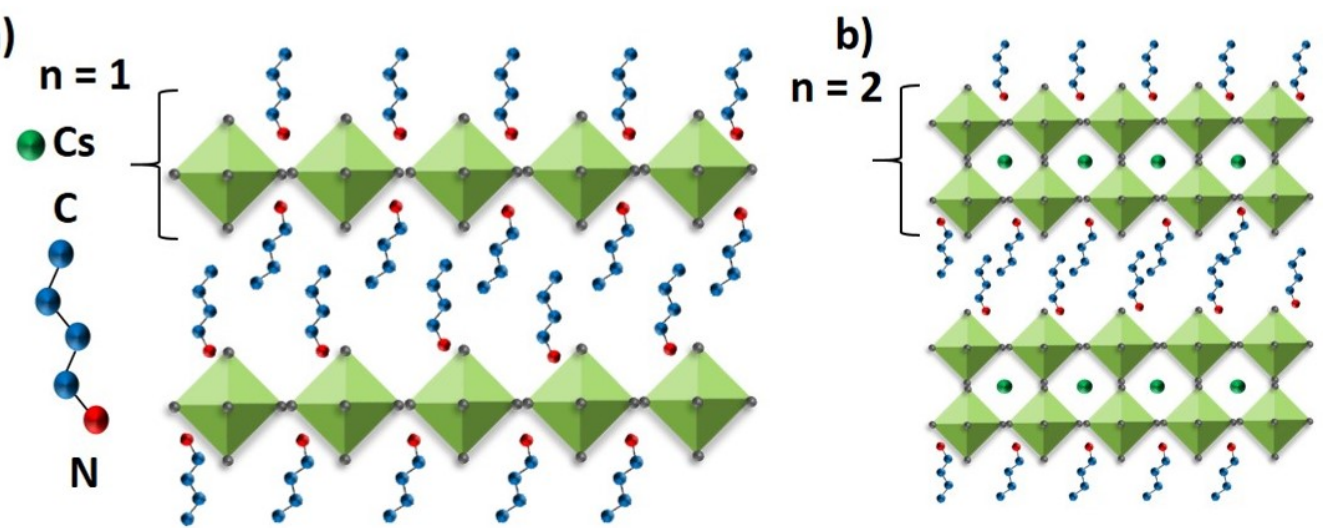

Figure 4.1: Illustrated structure of $B A_{2} C s_{n-1} P b_{n} X_{3 n+1}$ perovskites for, a) $n=1$, and b) $n=2$.

bility in ambient conditions and less colour tuneability [86, 87]. 2D perovskites were found to have remarkable colour purity and brightness. However, due to their larger exciton binding energy, they were found to be relatively inefficient $[35,36]$. The larger exciton binding energy was due to the geometric and dielectric constant in these 2D materials and thus more energy was required to achieve the adequate conduction for electrons [35, 36, 219]. Therefore, the binding energy needs to be relatively low for the optoelectronic applications. In all of these cases, including 3D, 2D or NC perovskites, the challenges were still remained.

To overcome this issue, a perovskite material, which has moderate binding energy, high PL quantum yield at lower excitation, less bulky ligands, and relatively higher stability is needed. A quasi-2D perovskite material, which has relatively higher number of planes, is an alternate solution [30-33, 133]. This crystal phase is called a Ruddlesden-Popper (RP) phase perovskite, which is a multi-layered phase of perovskite separated from each other by a cation. In this research, we are using an organic cation as a separating layer. Figure 4.1 shows the illustration of the crystal structure. When $n=1$, there is only one monolayer of perovskite octahedra connected with the next group using organic cation (butylammonium). However, when $n=2$, two monolayers of perovskite octa- 
hedra are connected together forming a multi-layered structure (Figure 4.1b). Each multi-layered structure is connected with its neighbouring nanosheets by a single layer organic cation (butylammonium). If number of monolayers, $\mathrm{n}=1$, then it it is a pure $2 \mathrm{D}$ structure. As number of monolayers, $\mathrm{n}$, increases the structure becomes more $3 \mathrm{D}$. When $\mathrm{n}$ is infinity then the structure is pure 3D polycrystalline. Thus, If number of monolayers are more than one then it forms a quasi-2D or RP phase perovskite structure. The mobility of charges were also found to be increased from $0.9 \mathrm{~cm}^{2} \mathrm{~V}^{-1} \mathrm{~s}-1$ to $6.5 \mathrm{~cm}^{2} \mathrm{~V}^{-1} \mathrm{~s}-1$ with the layer thickness from 1 to 5 in MAPI based quasi-2D perovskite thin films, as the Coulombic interaction between electron and hole reduces. This results in the lower binding energy $[37,220]$. The thicker materials weakens the binding energy of the excitons and thus combines the colour purity and tuneability of $2 \mathrm{D}$ structures with the conduction properties of 3D crystals [35-37].

Here, we synthesised RP phase perovskites with a formula of $\mathrm{A}_{2}^{\prime} \mathrm{A}_{n-1} \mathrm{~Pb}_{n} \mathrm{X}_{3 n+1}$, where $A^{\prime}$ is a large ammonium organic cation (butyl ammonium), $A$ is the monovalent inorganic cation $\left(\mathrm{Cs}^{+}\right)$, and $\mathrm{X}$ is a halogen $(\mathrm{Cl}, \mathrm{Br}, \mathrm{I}), \mathrm{n}$ is the number of monolayers of octahedra, which were between $\approx 16-40$ in the structure. The larger band gap organic cation (A') works as an insulating layer or a barrier between $\mathrm{PbI}_{6}{ }^{-}$octahedra (Figure 4.1). In addition, varying the the number of inorganic perovskite layers (n) between the larger cation ( $\left.A^{\prime}\right)$, it should be possible to tune the optical properties, such as band gap and emission [32]. The optical properties can also be tuned by mixing the halide anions $(\mathrm{Br} / \mathrm{I}$, $\mathrm{Br} / \mathrm{Cl}$ ) similar to Chapter 3 [221]. Thus, a tuneable emission spectra should be achieved in quasi-2D perovskites similar to other perovskite materials by using composition and the degree of confinement (monolayer thickness). The moisture stability of methylammonium lead halide and formamidinium lead halide perovskite solar cells and LEDs were found to be improved using A' group cations, such as phenylethylamine or 4-tertbutylamine [33, 222, 223]. Chang et al. produced the RP perovskite phase $\mathrm{CsPbI}_{3}$ LEDs, with $7.3 \% \mathrm{EQE}$, using 1naphthylmethylammonium(NMA) cations as A' group [224]. Shang et al. produced pure $\mathrm{CsPbBr} \mathrm{Pb}_{3} \mathrm{RP}$ phased perovskite LEDs using phenylethylammonium 
(PEA) as A' group and reported EQE of $4.5 \%$ at $501 \mathrm{~nm}$ [28]. Cheng et al. also produced inorganic RP perovskite phase LEDs emitting at $491 \mathrm{~nm}$ with $0.01 \%$ EQE [39]. Si et al. reported pure inorganic $\mathrm{CsPbBr}_{3}$ and $\mathrm{CsPbI}_{3}$ based RP perovskite LEDs using phenylbutylammonium (PBA) as the A' group with EL peak at $514 \mathrm{~nm}$ for $\mathrm{CsPbBr}_{3}$ and $683 \mathrm{~nm}$ for $\mathrm{CsPbI}_{3}$ [30]. However, there is no report on precise colour tuning in caesium based pure inorganic RP perovskite LEDs. Whereas, $\mathrm{CsPbBr}_{3} \mathrm{RP}$ perovskite LEDs displayed high EQE ( $\left.\approx 10 \%\right)$ in the green spectral region, efficient LEDs emitting in the pure blue spectral region (460-490 nm) were yet to be produced [30,39]. Blue LEDs are generally more difficult to produce as they require even precise tuning of the band gap $[39,225]$.

This chapter includes the synthesis of colour tuneable in-situ grown RP phase $\mathrm{CsPb}(\mathrm{Br} / \mathrm{Y})_{3}(\mathrm{Y}=\mathrm{Cl}, \mathrm{I})$ perovskite nanosheets using butylammonium (BA) as the $A^{\prime}$ group. The emission wavelength is tuned by varying the number of layers (n) (Figure 4.1) and halogen composition [221] [32, 131]. The RP perovskite crystals were grown by a solution processing technique in a nitrogen glove box. The chemical formula of the RP perovskite layer is $\mathrm{BA}_{2} \mathrm{Cs}_{n-1} \mathrm{~Pb}_{n}(\mathrm{Br} / \mathrm{Y})_{3 n+1}(\mathrm{Y}=\mathrm{Cl}$, I). The chapter further shows the use of these inorganic RP phased perovskites in light emitting diodes (LEDs). For the first time, we demonstrated the RP perovskite LEDs with precise colour tuning in the entire visible spectrum from 465 to $680 \mathrm{~nm}$. The LEDs produced by this material showed high EQE in the blue and green spectral region and low EQE $(<0.5 \%)$ in the red spectral region. LEDs in the blue regions showed peak EQE of $2.4 \%$ and $6.2 \%$ at 465 and $487 \mathrm{~nm}$ EL wavelength, which is the best reported blue perovskite LED to date. In addition, we also produced a blue-green device, emitting at $506 \mathrm{~nm}$, with best overall EQE of $10.1 \%$. 


\subsection{Experimental}

This section includes the synthesis of caesium based RP perovskite followed by the fabrication of LEDs. The fabrication process includes the spin coating and thermal evaporation techniques. It also includes the method of electrical and optical characterisation, which includes the use of spectrometer for EL measurement and Keithley source measurement unit for electrical characterisation.

\subsubsection{Materials}

TPBi LT-E302 $\left(\mathrm{MW}=654.76 \mathrm{~g} \cdot \mathrm{mol}^{-1}\right)$ and PVK LT-N4077 $\left(\mathrm{MW}>20000 \mathrm{~g} \cdot \mathrm{mol}^{-1}\right)$ were purchased from Lumtec. PEDOT:PSS (AL4083) was purchased from Ossila. N-butylammonium bromide and n-butyl ammonium iodide were purchased from GreatCell Solar. caesium bromide, caesium iodide, lead(II) chloride, lead(II) bromide, lead(II) iodide, anhydrus dimethyl sulfoxide (DMSO), anhydrus chlorobenzene, m-xylene, and chloroform were purchased from Aldrich. ITO coated glass substrates were purchased from Kintec.

\subsubsection{Method}

$\mathrm{CsPbX}_{3} \mathrm{RP}$ perovskite were prepared in-situ on ITO coated glass film. The synthesis method involved the preparation of perovskite precursor solution followed by spin coating of the precursor solution on a substrate. Appropriate hole and electron transport layers were also spin coated to fabricate perovskite LEDs. 


\subsubsection{Synthesis of Perovskite Precursor Solution}

The precursor solution was prepared by mixing n-butylammonium bromide (9.5 mg), lead(II) bromide (31.7 mg), and caesium bromide (23.1 mg) in $1 \mathrm{~mL}$ of anhydrus DMSO at room temperature under $\mathrm{N}_{2}$ glove box. In the case of mixed halide certain ratio of chloride or iodide precursors were mixed with bromide precursor to synthesise the mixed halide precursor solution (Table 4.1).

\subsubsection{Fabrication of LEDs}

Similar to Chapter 3, a $12 \mathrm{~mm}$ x $12 \mathrm{~mm}$ ITO coated substrate was used to fabricate the devices. The device was fabricated on top of ITO coated film and which sandwiched between ITO back electrode and Al top electrode. Section 3.2.1.2 explains the device schematic in details.

Patterned ITO substrates were used as an anode due to its high conductivity and transparency. Substrates were cleaned by the standard process of ultra sonicating for $20 \mathrm{~min}$ in soap solution, distilled water, acetone and isopropanol successively. They were then heated under vacuum for $30 \mathrm{~min}$ followed by four min oxygen plasma cleaning.

LEDs were fabricated using a common device architecture of QD LEDs with some slight modification [1]. Where, PEDOT:PSS, poly-TPD, and PVK were used as a hole transport layer and TPBi were used as an electron transport layer. poly-TPD $(8 \mathrm{mg} / \mathrm{mL})$ and PVK $(10 \mathrm{mg} / \mathrm{mL})$ were dissolved in chlorobenzne. PEDOT:PSS was already dispersed in water from the supplier. PEDOT:PSS was filtered through polyvinylidene fluoride (PVDF) filters $(0.45 \mu \mathrm{m})$ and then spincoated onto the clean ITO-coated glass substrates at $4000 \mathrm{rpm}$ for $60 \mathrm{~s}$ followed by annealing at $145^{\circ} \mathrm{C}$ in air for $20 \mathrm{~min}$. Substrates were transferred in the nitrogen glove box. The hole transporting layer, PVK (in chlorobenzene, $10 \mathrm{mg} / \mathrm{mL}$ ), was spin-coated onto the PEDOT:PSS layer at $3000 \mathrm{rpm}$ for $60 \mathrm{~s}$ and annealed 
Table 4.1: A table for precursor concentration to synthesise perovskites solution. These quantities produced $1 \mathrm{~mL}$ of precursor solution in DMSO.

\begin{tabular}{|c|c|c|c|c|c|c|}
\hline $\begin{array}{c}\text { PL emission } \\
(\mathbf{n m})\end{array}$ & $\begin{array}{c}\mathbf{B A B r} \\
(\mathbf{m g})\end{array}$ & $\begin{array}{c}\mathbf{C s B r} \\
(\mathbf{m g})\end{array}$ & $\begin{array}{c}\mathbf{P b B r} \\
(\mathbf{m g})\end{array}$ & $\begin{array}{c}\mathbf{B A I} / \mathbf{B A C l} \\
(\mathbf{m g})\end{array}$ & $\begin{array}{c}\mathbf{C s I} \mathbf{C s} \mathbf{C l} \mathbf{( m g}) \\
\mathbf{P b I}_{2} / \mathbf{P b C l}_{2} \\
(\mathbf{m g})\end{array}$ \\
\hline 465 & 9.5 & 23.1 & 3.1 & 0 & 0 & 21.5 \\
486 & 9.5 & 23.1 & 18.9 & 0 & 0 & 9.5 \\
500 & 17.1 & 23.1 & 31.7 & 0 & 0 & 0 \\
505 & 9.5 & 23.1 & 31.7 & 0 & 0 & 0 \\
514 & 4.8 & 23.1 & 31.7 & 0 & 0 & 0 \\
546 & 6.6 & 16.1 & 22.1 & 3.7 & 8.4 & 11.7 \\
590 & 5.7 & 13.9 & 19.0 & 4.9 & 11.2 & 15.6 \\
641 & 4.3 & 10.3 & 14.2 & 6.8 & 15.4 & 21.4 \\
681 & 1.0 & 2.3 & 3.1 & 11.2 & 25.2 & 35.1 \\
\hline
\end{tabular}

at $140{ }^{\circ} \mathrm{C}$ for $30 \mathrm{~min}$. The perovskite precursor solution was spin-coated at 5000 rpm for $60 \mathrm{~s}$ followed by annealing at $100{ }^{\circ} \mathrm{C}$ for better crystallisation. Devices were then moved to the thermal evaporator for the deposition of a $40 \mathrm{~nm}$ TPBi electron transport layer of TPBi. A $100 \mathrm{~nm} \mathrm{Al} \mathrm{electrode} \mathrm{deposition} \mathrm{was} \mathrm{achieved}$ by thermal evaporation through a shadow mask. Then a thin layer of Ag electrode was deposited on top of $\mathrm{Al}$ to prevent the $\mathrm{Al}$ oxidation. For $\mathrm{CsPb}(\mathrm{Br} / \mathrm{I})_{3}$ LEDs, a thin layer of poly-TPD (in m-xylene, $8 \mathrm{mg} / \mathrm{mL}$ ) was spin coated at 4000 RPM on top of PEDOT:PSS followed by annealing at $120^{\circ} \mathrm{C}$ for $20 \mathrm{~min}$ and then a very thin layer of PVK (in chlorobenzene, $5 \mathrm{mg} / \mathrm{mL}$ ) was spin coated at 4000 RPM on top of poly-TPD for the compatibility of perovskite film, as the perovskite films formed a rough surface on top of poly-TPD. The PVK film was annealed the same way at $140^{\circ} \mathrm{C}$ for $30 \mathrm{~min}$ and then the perovskite layer was spin coated as described above. A clear device schematic is shown in Figure 3.2 with the estimated thickness of each layer. Energy band diagram shows the energy level relative to the vacuum and the estimation of energy level was taken from 
the literature [130, 200, 201].

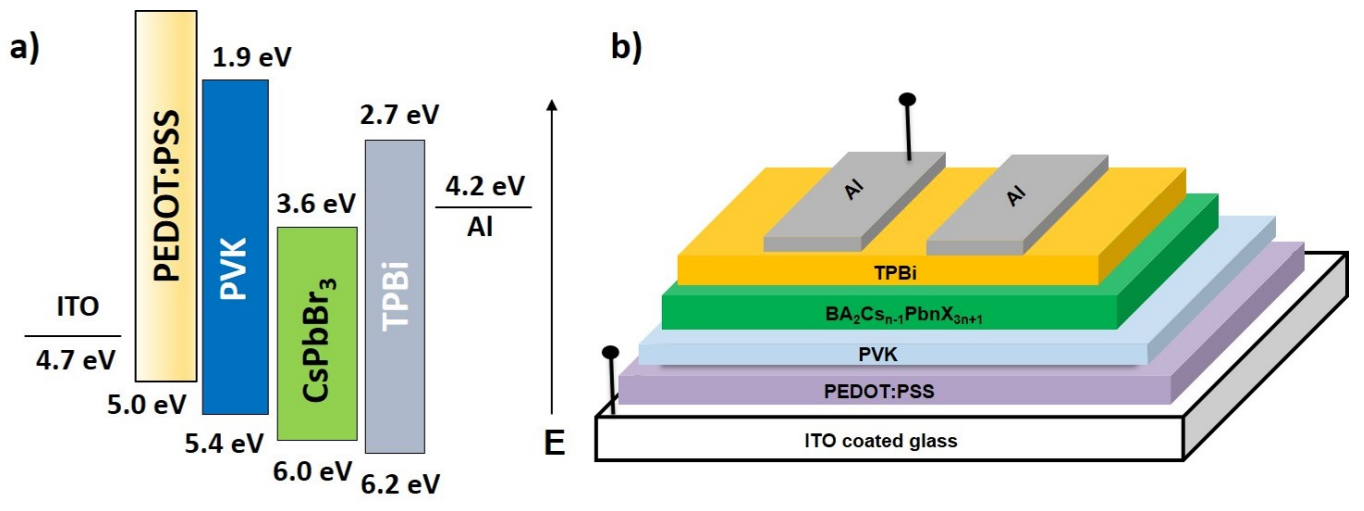

Figure 4.2: a) Device schematic and b) energy diagram for the ITO/PEDOT:PSS/PVK/CsPbBr $r_{3} R P$ perouskite/TPBi/Al LEDs. In order to align the band positions for hole injection, A poly-TPD layers was used in between PEDOT:PSS and PVK layer for $\mathrm{CsPb}(\mathrm{Br} / \mathrm{I})_{3} \mathrm{LEDs}$, as poly-TPD has a lower valancce band position (5.43 eV) for hole injection (Figure 3.2).

\subsubsection{LED Measurement}

The corner of the device was carefully scrubbed by chloroform in order to connect the probe with ITO electrode. A Keithley 236 source measurement unit (SMU) was used as a power source for the LED. Positive terminal of power source was connected with ITO and negative terminal was connected with Al electrode. The input voltage was slowly ramped up from $0 \mathrm{~V}$ and the EL spectrum was recorded at certain voltages or time intervals after the turn-on voltage. All devices were characterised in ambient conditions using a QE pro Ocean Optics spectrometer connected with a $200 \mu \mathrm{m}$ optical fiber (QP200-2VIS-NIR). The fiber was carefully calibrated with an Ocean Optics HL-3 plus VIS-NIR light source. A Lambertian profile was observed and assumed in the EQE calculation $[34,158]$ 


\subsection{Results and Discussion}

The RP perovskite films were structurally characterised by TEM, SEM, and XRD. TEM helps to determine the morphology with a rough estimation on number of layers of quasi-2D perovskites. Optical properties, such as emission and absorption studies, were characterised by UV-Vis and PL spectrometer. The EL along with the IV measurements were carried out to calculate the device metrics such as, EQE (\%), luminance $\left(\mathrm{cd} / \mathrm{m}^{2}\right)$, luminous efficacy $(\mathrm{lm} / \mathrm{W})$, and current efficiency (cd/A).

\subsubsection{Morphological Characterisation of RP Perovskite}

TEM samples were prepared by extracting the perovskite nanosheets from the ITO substrate on which they were grown on top of PEDOT:PSS and PVK layer. The substrate was dipped in an anhydrous chloroform solvent followed by ultrasonication for five min. Chloroform dispersed the nanosheets and dissolved the underlying organic layers. The resulting solution was drop casted on a Cu200 mesh on forvar grid for TEM measurements.

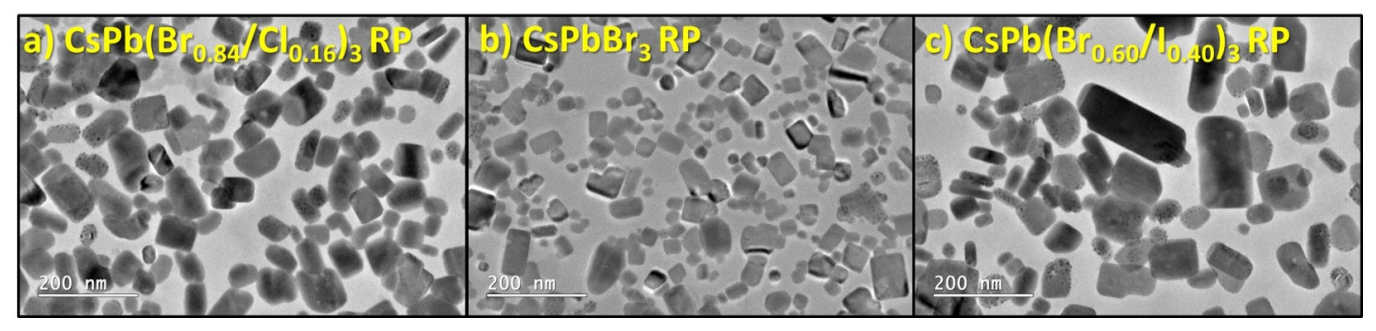

Figure 4.3: TEM images of a) $B A_{2} C s_{n-1} P b_{n}(B r / C l)_{3 n+1}$, b) $B A_{2} C s_{n-1} P b_{n} B r_{3 n+1}$, and c) $B A_{2} C s_{n-1} P b_{n}(B r / I)_{3 n+1} R P$ perouskites nanosheets after removal from the substrate. The loading ratio of each halogen is labelled on top of each TEM. The samples were prepared by drop-casting chloroform nanosheets suspension on a $\mathrm{Cu}$-200 mesh on formvar grid.

Only $\mathrm{BA}_{2} \mathrm{Cs}_{n-1} \mathrm{~Pb}_{n}(\mathrm{Br} / \mathrm{Cl})_{3 n+1}, \mathrm{BA}_{2} \mathrm{Cs}_{n-1} \mathrm{~Pb}_{n} \mathrm{Br}_{3 n+1}$, and $\mathrm{BA}_{2} \mathrm{Cs}_{n-1} \mathrm{~Pb}_{n}(\mathrm{Br} / \mathrm{I})_{3 n+1}$ 
were characterised to observe the morphology, as the similar methodology was conducted to synthesise all of the mixed halide samples. Figure 4.3 shows the TEM image of nanosheets. As can seen that all of the samples consist entirely of nanosheets. In TEM images, the size of nanosheets are found to be widely distributed because some of the nanosheets appeared to be from the top view, whereas some of them were found to be tilted at the side. Therefore, the size distribution on side angle nanosheets were analysed. High resolution TEM analysis (Figure 4.4) on side angle nanosheets showed the reasonable size dispersion.

To estimate the number of layers, sample was looked at higher resolution. But, It was relatively difficult to observe the clear lattice fringes as the sample had underlying organic layers on the TEM grid and was also found to be melting with electron beam. Figure 4.4 shows the high resolution TEM images of $\mathrm{CsPbBr}_{3}$ RP perovskite sample. A side view of single nanosheet can be seen in the TEM micrographs, which illustrates the lattice fringes with d spacings of $5.9 \AA$ in all of the nanosheets. The number of layers were estimated by measuring the total number of lattice fringes in each side viewed nanosheet. The number of planes were found to be varying from 17 to 40 and the mean number of planes (n) for the sample was estimated to be $\approx 24 \pm 7$ monolayers. The bottom of Figure 4.4 shows the illustration of RP perovskite structure when the number of layers is 24. As one can compare this from Figure 4.1, where the number of layers is only $1-2$, which shows the increase in number of layers lead to a thicker nanosheet. TEM micrographs also suggest the same concept, the thickness of nanosheet is $10.6 \mathrm{~nm}$ for $\mathrm{n}=17$, however, for $\mathrm{n}=40$ the thickness changes to $23.4 \mathrm{~nm}$ (Figure 4.4). Thus, the TEM analysis confirms the formation of RP perovskite phase, where the number of layers are in the range of 16-40.

X-ray diffraction measurements were taken with a PANalytical X-Ray diffractometer using $\mathrm{Cu}-\mathrm{K} \alpha$ radiation on a spinner stage, with an operation voltage of $45 \mathrm{kV}$ and current of $40 \mathrm{~mA}$. The same samples were used for XRD analysis prior to use for TEM measurements. Figure 4.5 shows the XRD spectra of 

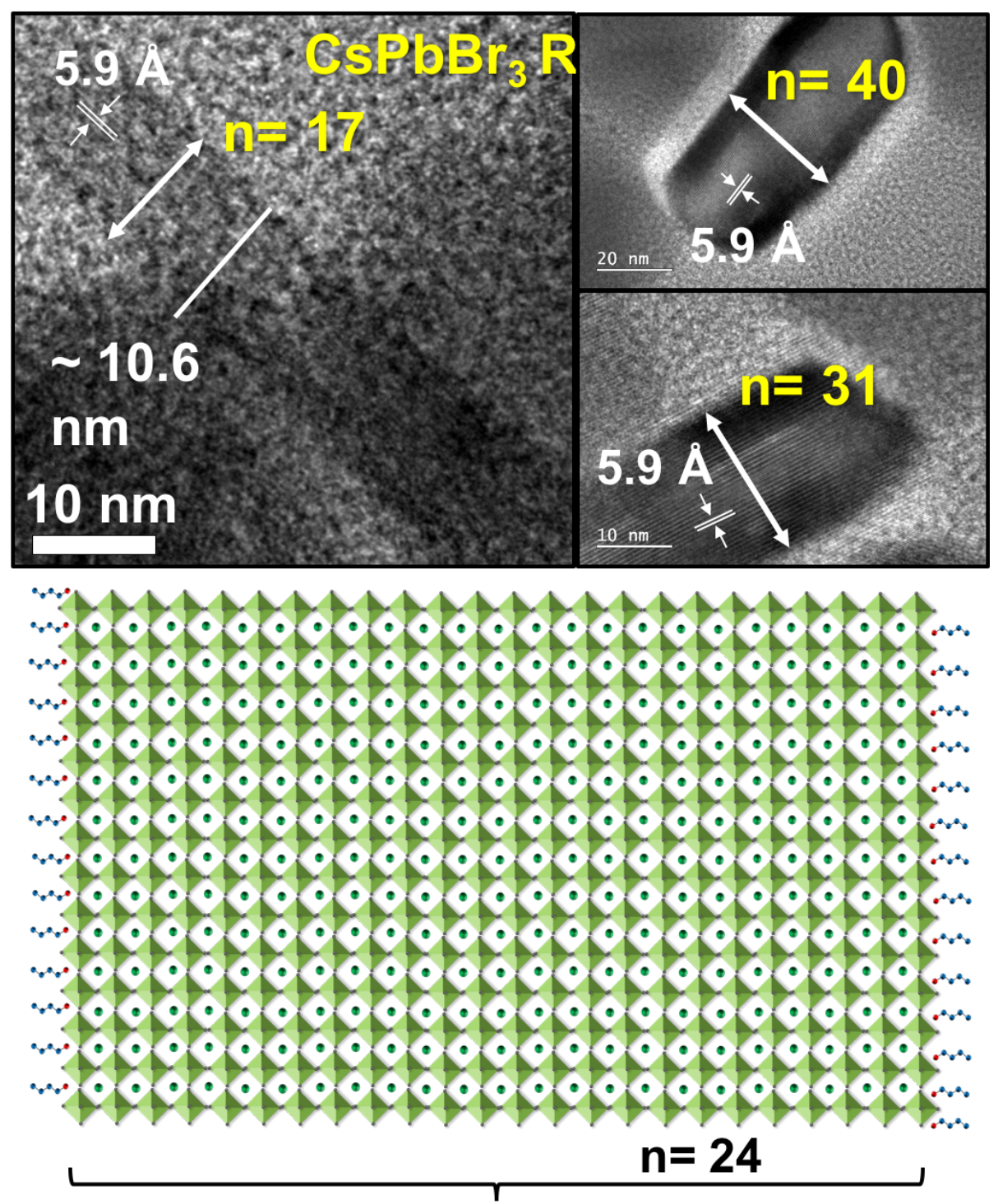

Figure 4.4: Top) High resolution TEM images of $B A_{2} C s_{n-1} \mathrm{~Pb}_{n} B r_{3 n+1}$ nanosheets. The number of planes can be observed in the material from a side view of a single nanosheet and were found to be between 17 to 40. The samples were prepared by drop-casting chloroform nanosheets suspension on a Cu-200 mesh on formvar grid. Bottom) An Illustrated structure of $\mathrm{BA}_{2} \mathrm{Cs}_{n-1} \mathrm{PbnX}_{3 n+1}$ perouskite for $n=24$, which shows the increment in number of planes with nanosheet thickness. 


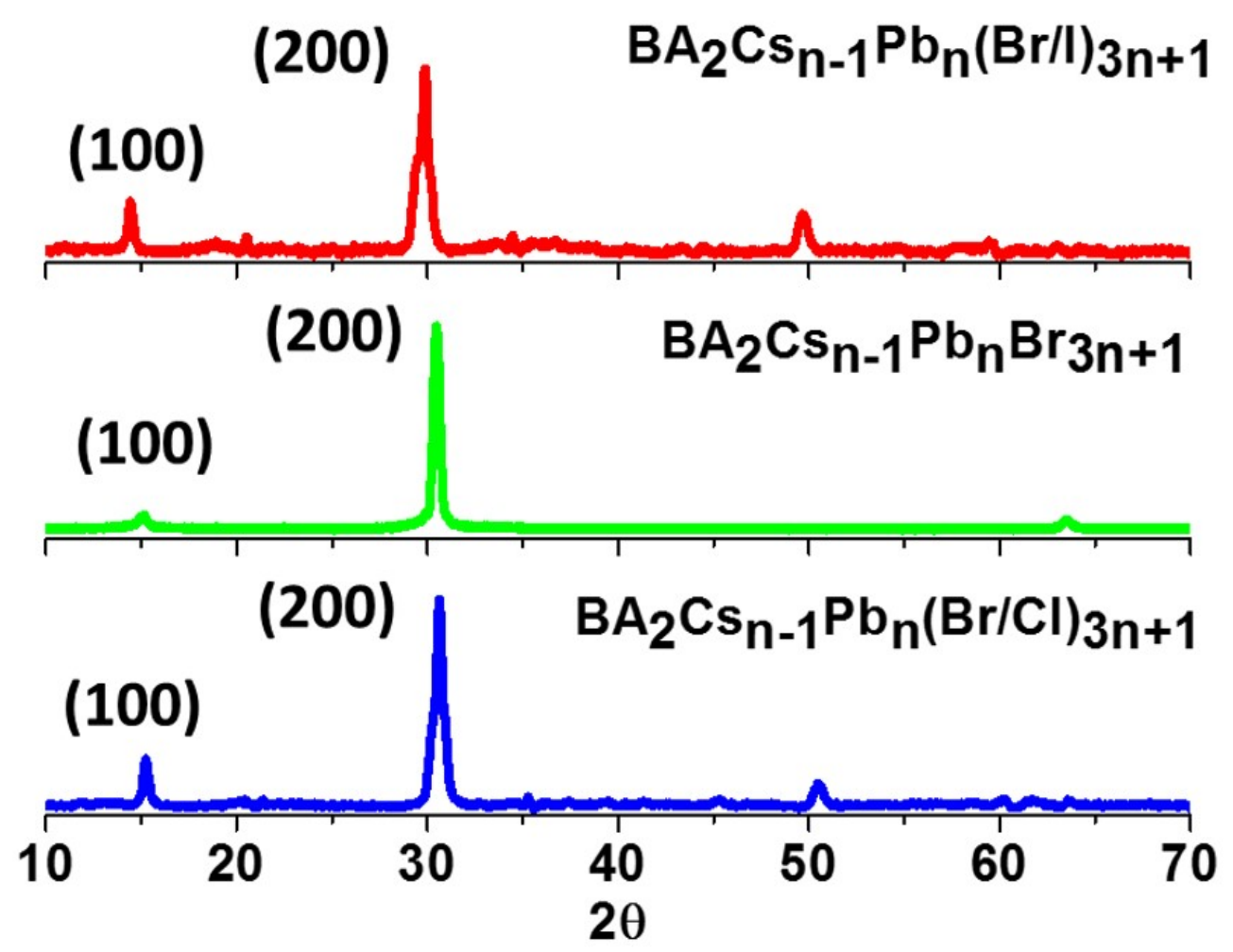

Figure 4.5: XRD spectra of $B A_{2} C s_{n-1} P b n(B r / Y)_{3 n+1}, Y=C l$ or I. Perouskite thin films were spin coated onto PVK and PEDOT:PSS for the XRD measurements. Low angle XRD peaks $(2 \theta<10)$ were absent as $n$ was larger than 16 in these $R P$ perovskite thin films.

$\mathrm{BA}_{2} \mathrm{Cs}_{n-1} \mathrm{Pbn}(\mathrm{X} / \mathrm{Y})_{3 n+1}, \mathrm{Y}=\mathrm{Cl}$ or I nanosheets thin film. The pure $\mathrm{CsPbBr} \mathrm{XRD}_{3}$ spectrum shows the diffraction peaks at $15.1^{\circ}$ and $30.4^{\circ}$ which correspond to the (010) and (020) planes of perovskite NCs. [30]. A similar trend was observed in the mixed halide, however the diffraction peak was shifted towards lower angles in the case of bromide-iodide mixed halides and towards a higher angle for bromide-chloride mixed halide perovskites. Lower angle diffraction peaks, consistent with phase pure and thin nanosheets, were not expected to be observed as the number of planes was higher than 16, on average [30]. XRD peaks 
were broader than bulk spectra of $\mathrm{CsPbX}_{3}$ as per the Scherrer equation (1.39). Thus, the X-ray diffraction analysis confirmed the formation of perovskite crystal structure and also the tuneability of mixed halides.

\subsubsection{Optical Characterisation of RP Perovskite Thin Films}

Optical spectroscopy can provide the details of the materials' band gap and emission properties. It can help us in observing the effect of halide composition or confinement effect in the band gap and PL properties. A Cary 50 UV-Visible spectrophotometer was used to do the UV-Vis spectroscopy and a Horiba Fluorolog-3 Spectrometer were used for the PL measurement. Perovskite thin films coated on ITO/PEDOT:PSS/PVK were tested for the optical characterisation. A baseline was created using ITO/PEDOT:PSS/PVK thin film. All of the samples are labelled with their respective emission peaks.

Figure 4.6 shows the absorption spectra of RP phase perovskite thin films. All of the samples showed the broad absorption edge, which was consistent with previously reported RP perovskite thin films [30]. The broad absorption edge shifts to the blue from R681 to B465, as the halide mix goes from high I/low Br to pure $\mathrm{Br}$, to high $\mathrm{Cl} /$ low $\mathrm{Br}$. That means incorporation of I shifts the absorption edge towards the higher wavelength and incorporation of $\mathrm{Cl}$ shifts the absorption edge towards the lower wavelength. The similar feature was observed with halide mixing in Chapter 3 as well. Additionally, shifts in G506 and G513 samples were achieved by altering the BA concentration. Device G513 contained only $50 \%$ of butylammonium bromide (BABr), which results in thicker nanosheets and therefore a shift in absorption edge towards higher wavelength (Table 4.1). We were only interested to observe the change in absorption properties with varying the halide composition and number of layers. A detailed absorption study was not required to fabricate the LED and could be the part of future research. 


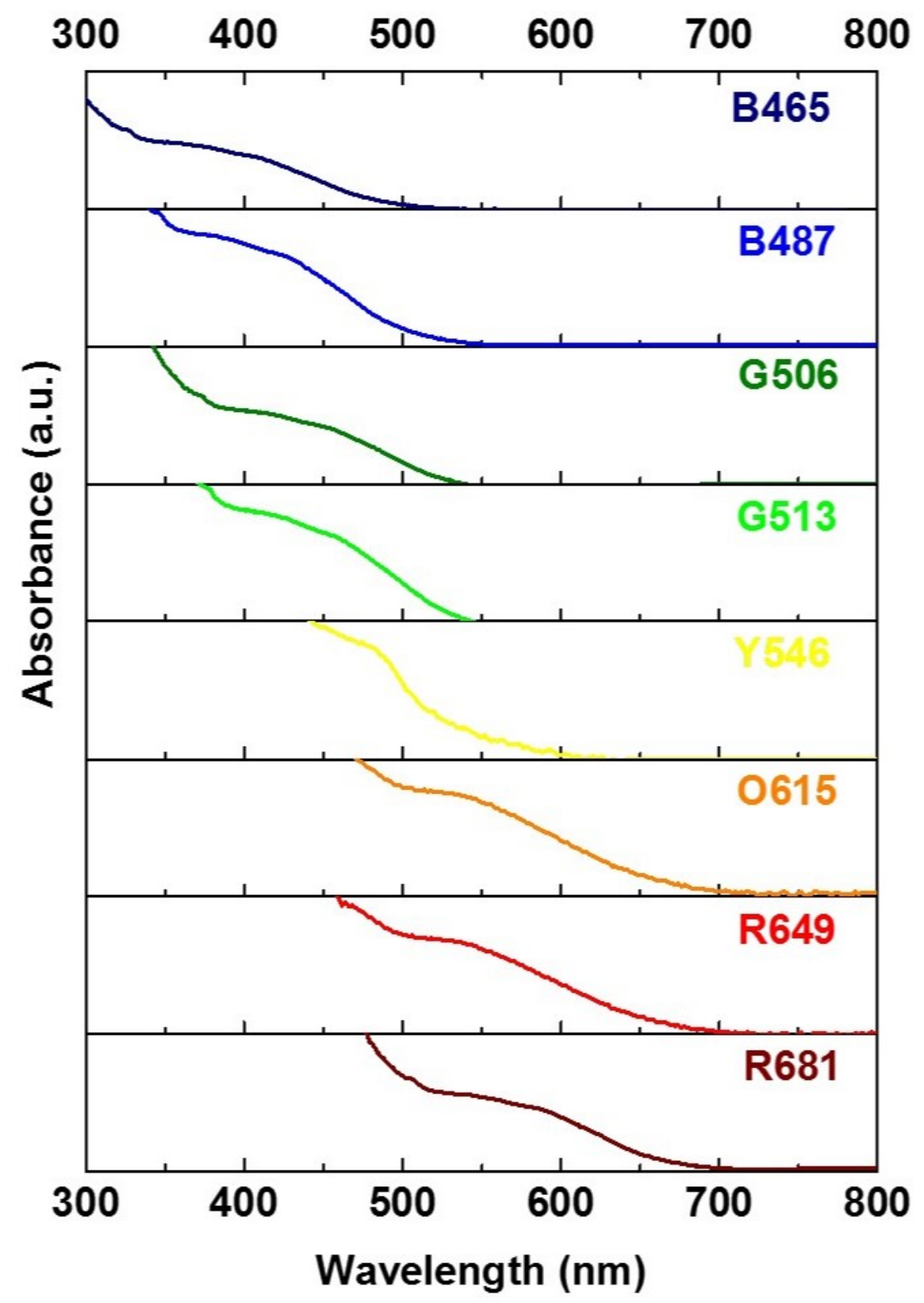

Figure 4.6: A UV-Vis absorption spectra of $B A_{2} C s_{n-1} P b_{n}(B r / Y)_{3 n+1},(Y=C l, I) R P$ perovskite thin films. Similar features have been reported in the literature for other $\mathrm{CsPbX}_{3} \mathrm{RP}$ perouskite thin films [30] 
Where as the absorption spectra have shown the dependence on halide composition and confinement. Similar behaviour should also be expected from the PL spectroscopy, i.e. the change in emission properties. Figure 4.7 shows the emission spectra of $\mathrm{CsPb}(\mathrm{X} / \mathrm{Y})_{3} \mathrm{RP}$ perovskite thin films in the entire visible spectrum. In Figure 4.7, it can be seen that the emission peak is tuned by changing the halide composition and BA concentration. That means incorporation of I shifts the emission peak towards the higher wavelength and incorporation of $\mathrm{Cl}$ shifts the emission peak towards the lower wavelength. B465 has a higher content of $\mathrm{Cl}$ therefore it shows the bluest emission. As the $\mathrm{Cl}$ content decreased (B487) the emission shifted towards the higher wavelength and the emission peak was found to be at $487 \mathrm{~nm}$. When there was no $\mathrm{Cl}$ in the thin film the emission was found to be at 505 and $514 \mathrm{~nm}$. A similar feature was observed by varying a $\mathrm{I} / \mathrm{Br}$ ratio such that samples with higher iodide content showed the emission at higher wavelength $(\approx 680 \mathrm{~nm})$. As the iodide content decreased, the emission was found to be blue-shifted and sample with the lowest amount of iodide showed emission at $546 \mathrm{~nm}$ (Table 4.1). Thus, similar to perovskite NCs, halide composition tuning had a significant effect on the emission properties of RP perovskites.

To a degree, the emission properties were also able to be tuned by changing the butylammonium halide concentration. In Figure 4.7, emission spectra ranging from $500-514 \mathrm{~nm}$, showing colour tuning by changing the BABr concentration. The emission peak was found to be at $505 \mathrm{~nm}$ when the BABr concentration was similar to other samples. However, when the concentration was increased $1.8 \mathrm{x}$, the emission peak blue-shifted to $500 \mathrm{~nm}$ and when the concentration was decreased to $0.5 \mathrm{x}$, the emission peak red-shifted to $514 \mathrm{~nm}$. The change in emission peak with BABr concentration alters the number of monolayers (n) in nanosheets $[32,35,36]$. This can be attributed to the fact that the number of inorganic perovskite layers ( $\mathrm{n}$ ) between the large organic cation $\left(A^{\prime}\right)$ alter the degree of excitonic confinement in each nanosheet (Figure 4.4) [35]. Thus, colour tuning was also achieved by confinement effect albeit to a lesser degree. Overall, by taking advantage of halide composition tuning and confinement effect, it 
was possible to tune the emission spectra across the entire visible region (465$680 \mathrm{~nm}$ ). All of these RP perovskite samples were then used to construct LEDs (Section 4.2.2.2).

\subsubsection{LED Performance}

The EL along with the IV measurements were carried out to calculate the device metrics such as, EQE, luminance $\left(\mathrm{cd} / \mathrm{m}^{2}\right)$, luminous efficacy $(\mathrm{lm} / \mathrm{W})$, and current efficiency (cd/A). All of the devices were measured in ambient conditions. Photographs of the rpp-LEDs at their turn on voltages (4.0-5.0 V) are

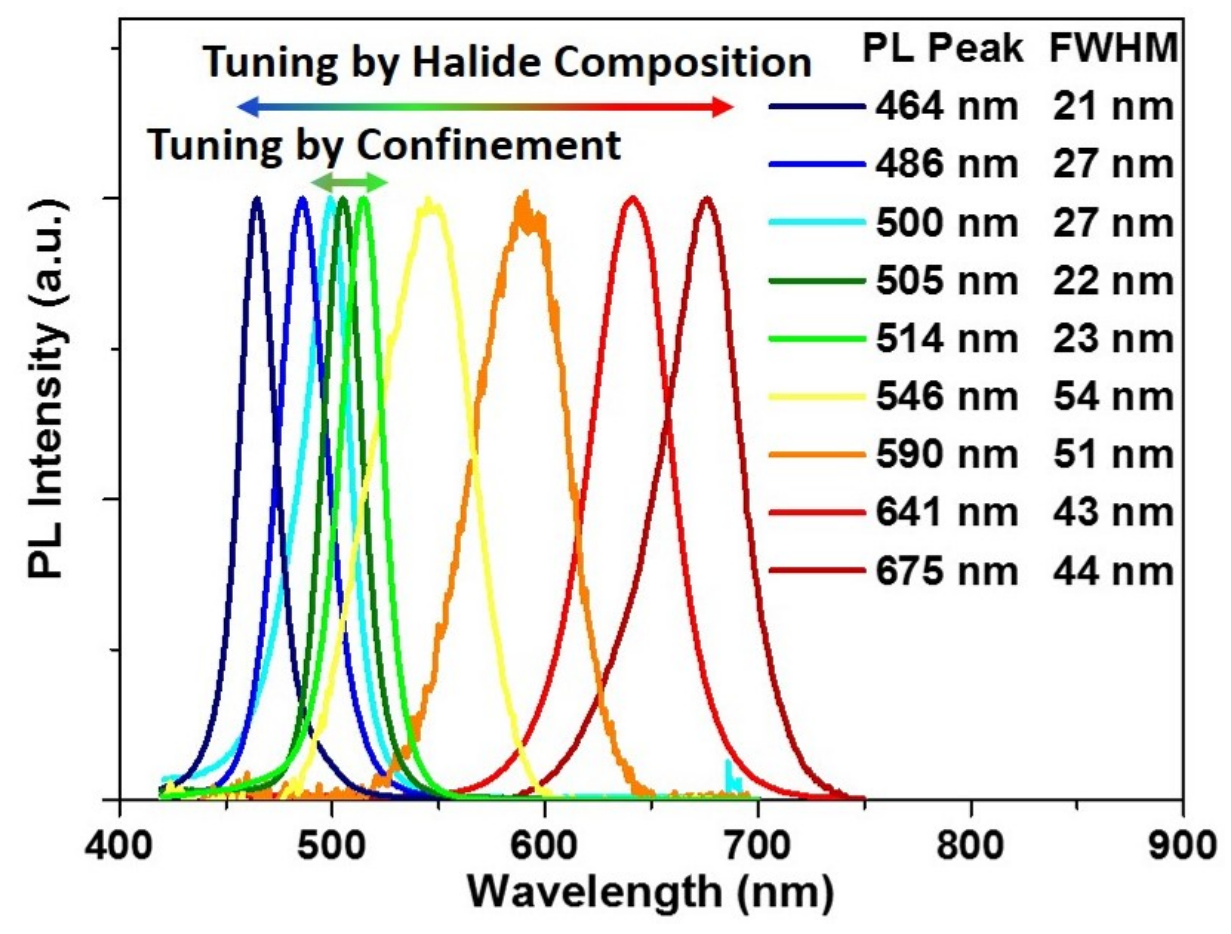

Figure 4.7: Normalised PL spectra taken at $400 \mathrm{~nm}$ excitation for $B A_{2} C s_{n-1} P b_{n}(B r / Y)_{3 n+1}(Y=C l, I) R P$ perovskite thin films. The colour tuning was achieved by varying the halide composition, as well as, BA concentration (Table 4.1). 

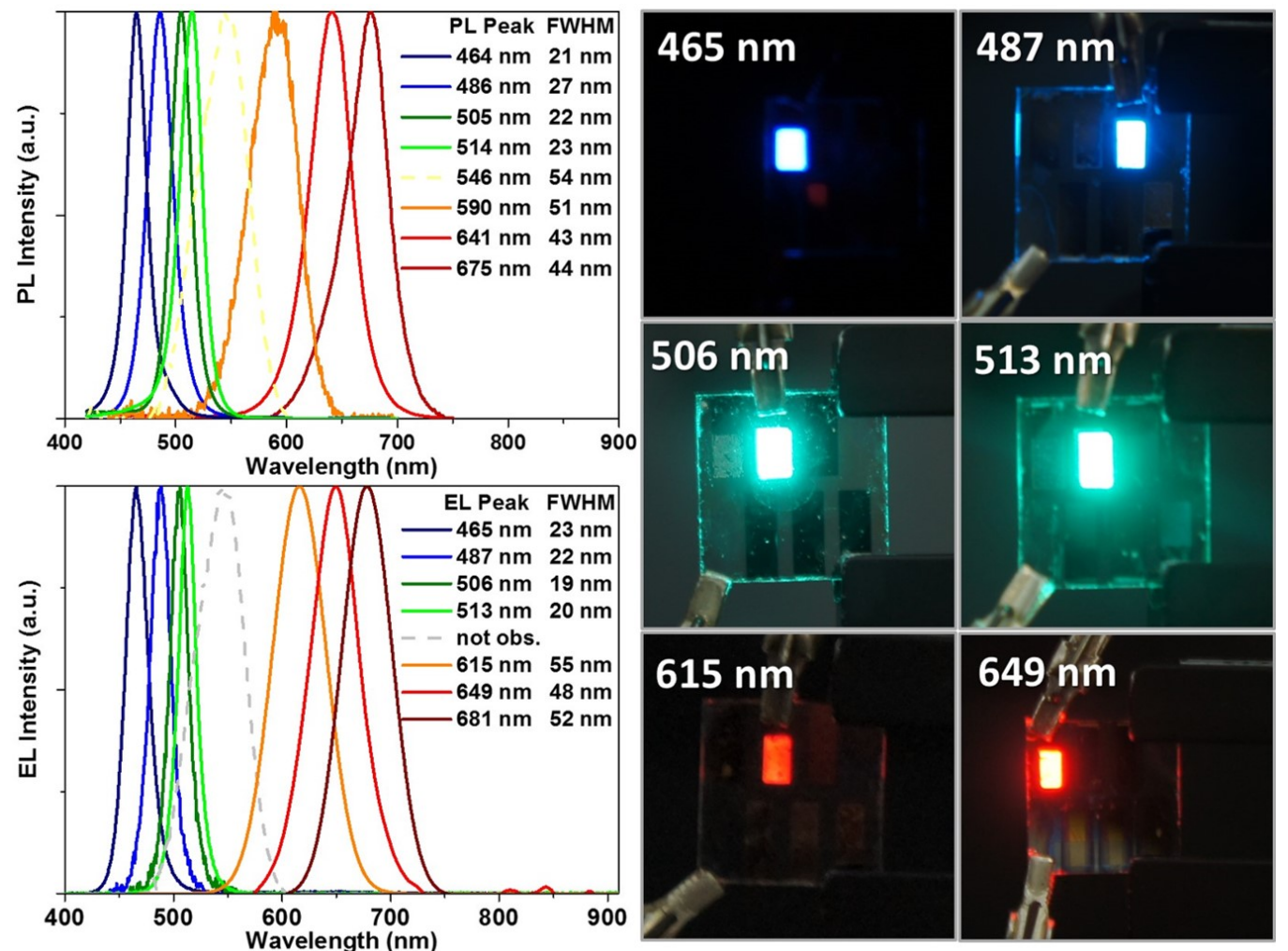

Figure 4.8: Normalised PL spectra, taken at $400 \mathrm{~nm}$ excitation $B A_{2} C s_{n-1} P b_{n}(X / Y)_{3 n+1}$, of $R P$ perovskite thin films in device (top left), unprocessed photos of LEDs (top right) and, normalised EL spectra (bottom left) of the same devices, all operating at turn-on voltage. The EL spectrum for the 546 nm device could not be observed at the same position due to ion migration.

shown in Figure 4.8. Figure 4.8 (top right) shows the PL spectra of rpp-LEDs. The corresponding EL spectra were recorded at early times near the their turnon voltages. PL and EL spectral peak positions with their FWHM are labelled in the graph. The EL position of each device is also labelled at the top of its photograph in Figure 4.8. Red emitting devices, $\mathrm{BA}_{2} \mathrm{Cs}_{n-1} \mathrm{~Pb}_{n}(\mathrm{Br} / \mathrm{I})_{3 n+1}$, were found to have relatively larger FWHM than blue-green devices. The EL peaks are similar to the PL peaks with a slight red shifted by few nanometers. The red-shift can be described to the transition from solution to thin films, where by Förster resonance energy transfer (FRET) occurs to neighbouring NCs with a 

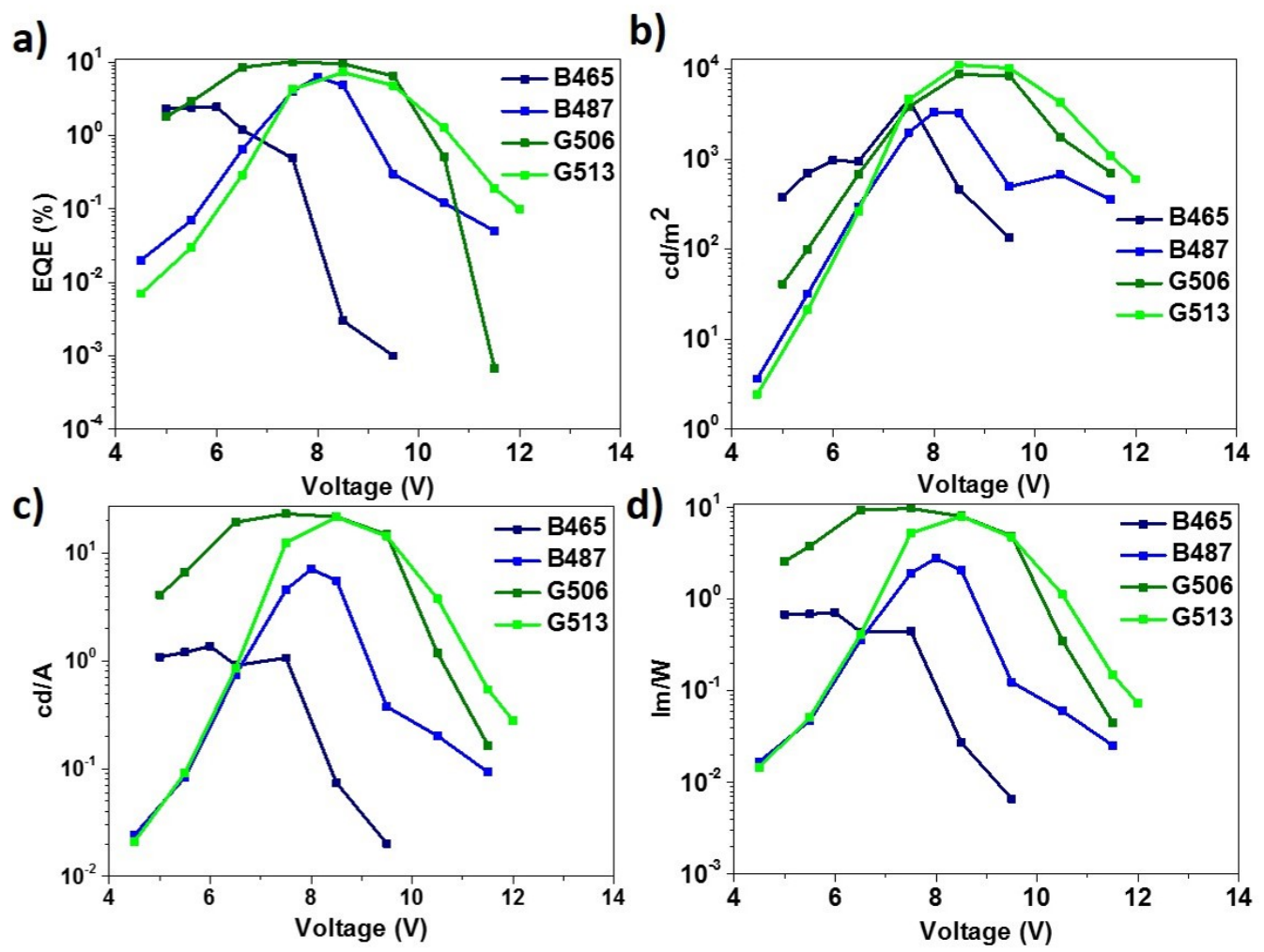

Figure 4.9: Chart showing a) EQE vs. voltage, b) luminance vs. voltage, c) current efficiency vs. voltage, and d) luminous efficacy vs. voltage in blue-green champion devices).

lower band gap. In FRET, excited state donor molecule transfer its energy to an another ground state acceptor molecule through a nonradiative process [202]. Similar to Chapter 3, no EL could be observed at $\approx 546 \mathrm{~nm}$, as will be discussed in next section.

Figure 4.9 shows the change in EQE, luminance, current efficiency, and luminous efficacy with voltage for blue-green LEDs. In all devices, except B465, the efficiency increased rapidly after their turn-on voltages and dropped off after 8.0 V. In B465, the maximum EQE was observed at the turn-on voltage $(5.0 \mathrm{~V})$. B465 showed the maximum luminance of $4620 \mathrm{~cd} / \mathrm{m}^{2}$ at $7.5 \mathrm{~V}$. B486 showed the highest EQE for a blue perovskite LED of $6.2 \%$ with the luminance was found 
to be $3340 \mathrm{~cd} / \mathrm{m}^{2}$ at $8.0 \mathrm{~V}$. Device G506 showed the highest EQE of $10.1 \%$ at $7.5 \mathrm{~V}$, however, the highest luminance of $8740 \mathrm{~cd} / \mathrm{m}^{2}$ with $9.4 \% \mathrm{EQE}$ was observed at $8.0 \mathrm{~V}$. B513 showed the EQE of $7.2 \%$ with $11,200 \mathrm{~cd} / \mathrm{m}^{2}$ luminance at 8.5 V. Efficiency roll-off at high current density was also observed for most of the devices. As the efficiencies were first found to be slightly increased with the current density then dropped down to the lower values (Figure 4.10).
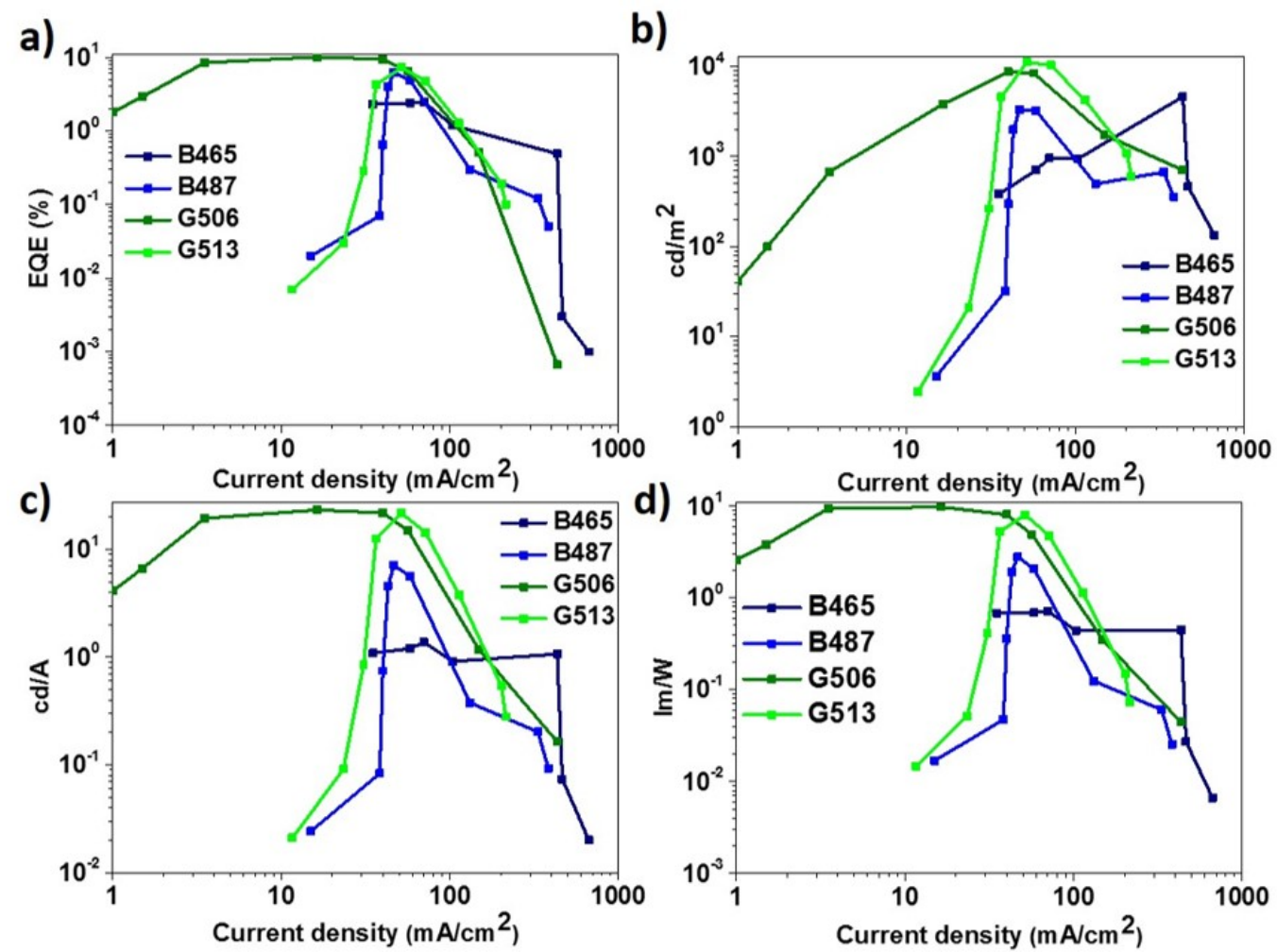

Figure 4.10: Chart showing a) EQE vs. current density, b) luminance vs. current density, c) current efficiency vs. current density, and d) luminous efficacy vs.current density in blue-green LEDs

Red devices were found to be relatively less efficient, it could be possibly due to either a) a very thin layer of PVK on top of poly-TPD, as PVK has a lower valence band position, which is not suitable for $\mathrm{CsPb}(\mathrm{Br} / \mathrm{I})_{3}$, or b) instability in $\mathrm{CsPb}(\mathrm{Br} / \mathrm{I})_{3}$ perovskites $[20,226]$. Figure 4.11 shows the change in efficiency with voltage for red devices. The turn-on voltage for each device was observed 

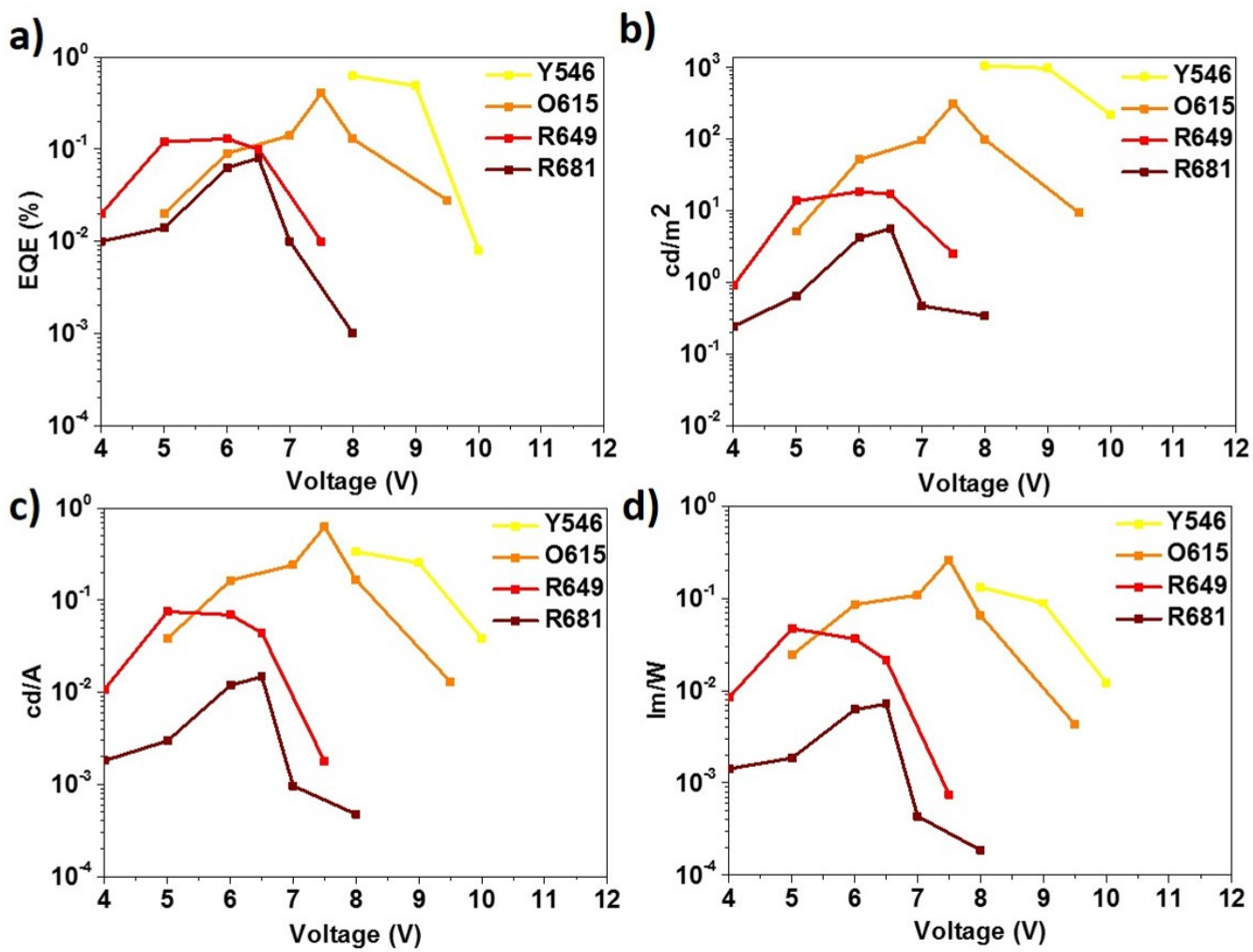

Figure 4.11: A plot of a) $E Q E$, b) $c d / m^{2}$, c) $c d / A$, and d) $l m / W$ vs voltage for $Y 546$, O615, R649, and R681 LEDs.

to be $4.0 \mathrm{~V}$, which is consistent with the J-V curve (Figure 4.12). The efficiency in these devices were much lower than blue or green devices. The, EQE, luminance $\left(\mathrm{cd} / \mathrm{m}^{2}\right)$, current efficiency (cd/A), and luminous efficacy $(\mathrm{lm} / \mathrm{W}) \mathrm{in}$ creased rapidly with the voltage until reaching about $\approx 7.0 \mathrm{~V}$ and then it started to decrease at high current density, similar to the blue and green LEDs. Device Y546 showed the initial EL position at $654 \mathrm{~nm}$ with $0.69 \%$ of EQE due to ion migration in mixed halide perovskites [20]. Device O615 showed the EQE of $0.4 \%$ at $7.5 \mathrm{~V}$ with the luminance of $313 \mathrm{~cd} / \mathrm{m}^{2}$. Device R649 showed the maximum EQE of $0.13 \%$ at $6.0 \mathrm{~V}$ with $18.5 \mathrm{~cd} / \mathrm{m}^{2}$ luminance. However, R681 showed the low EQE of $0.08 \%$ with the luminance of $5.6 \mathrm{~cd} / \mathrm{m}^{2}$. Thus, RP perovskite LEDs showed the EL in the entire visible spectrum. Table 4.2 shows the device metrics and statistic for all of the fabricated LEDs. It can be seen that 
Table 4.2: Device statistics for all of the fabricated rpp-LEDs. Blue and blue-green LEDs were found to be highly efficient. RED LEDs have shown low efficiency but still demonstrated the precise colour tuning in the pure red spectral region.

\begin{tabular}{|c|c|c|c|c|c|c|c|c|}
\hline $\begin{array}{l}\text { LED } \\
\text { label }\end{array}$ & $\begin{array}{c}\text { No. of } \\
\text { Devices }\end{array}$ & $\begin{array}{c}\text { EL peak } \\
(\mathbf{n m})\end{array}$ & $\begin{array}{l}\mathbf{V}_{t} \\
(\mathbf{V})\end{array}$ & $\begin{array}{c}\mathbf{E Q E}_{\max } \\
\mathbf{( \% )}\end{array}$ & $\begin{array}{c}\mathbf{E Q E}_{\text {avg }} \\
\mathbf{( \% )}\end{array}$ & $\begin{array}{c}\text { Std. Dev. } \\
\text { (EQEs) }\end{array}$ & $\begin{array}{l}\text { Luminance }{ }_{\text {avg }} \\
\quad\left(\mathbf{c d m}^{-2}\right)\end{array}$ & $\begin{array}{c}\text { Luminance at }_{\mathbf{E Q E}_{\text {max }}\left(\mathbf{c d m}^{-2}\right)} \\
\end{array}$ \\
\hline B465 & 8 & $465 \pm 3$ & $5.0 \pm 0.1$ & 2.40 & 2.09 & 0.23 & 471 & 510 \\
\hline B487 & 8 & $487 \pm 1$ & $4.5 \pm 0.5$ & 6.23 & 4.71 & 1.16 & 2320 & 3340 \\
\hline G506 & 7 & $506 \pm 1$ & $4.5 \pm 0.5$ & 10.1 & 8.93 & 1.21 & 3790 & 3810 \\
\hline G513 & 5 & $513 \pm 2$ & $4.5 \pm 0.3$ & 7.27 & 6.4 & 0.83 & 7390 & 11200 \\
\hline Y546 & 3 & $654 \pm 5$ & $8.0 \pm 2.0$ & 0.69 & 0.37 & 0.28 & 677 & 1070 \\
\hline 0615 & 3 & $615 \pm 5$ & $4.0 \pm 0.2$ & 0.41 & 0.35 & 0.06 & 254 & 310 \\
\hline R649 & 4 & $649 \pm 4$ & $4.0 \pm 0.7$ & 0.13 & 0.08 & 0.035 & 12 & 18 \\
\hline R681 & 3 & $681 \pm 4$ & $4.0 \pm 0.7$ & 0.08 & 0.05 & 0.03 & 3.6 & 5.7 \\
\hline
\end{tabular}

O615 was also slightly red shifted due to ion migration. The extent of ion migration reduced with the increase in iodide content in the perovskite structure. Thus, $\mathrm{CsPb}(\mathrm{Br} / \mathrm{I})_{3}$ LEDs with high bromide content, in particular, were found to be very colour unstable due to the extreme ion migration effect, a phenomena also previously observed in perovskite NC LEDs [20]. Devices with high iodide content were found to be more colour stable but showed very low EQE, possibly due to the phase instability of the $\mathrm{CsPbI}_{3}$ crystal structure [227].

Table 4.3 shows a comparison of our RP perovskite LEDs with some highly efficient RP perovskite LEDs, reported in the literature. Researchers have reported a variety of rpp-LEDs using different organic cations. However, no other report showed the colour tuneability through the entire visible region precisely using RP perovskite LEDs to the best of our knowledge (Table 4.3). Specifically, the emission in the blue (465-487 $\mathrm{nm}$ ) and pure red spectral region (615-649 nm) has not been reported yet (Table4.3). In addition, the blue LEDs, reported here, have outperformed the literature values $[28,39,128]$. Collectively, these RP perovskite LEDs have shown high EQE in blue-green spectral region and precise colour tuneability through the entire visible region. 
Table 4.3: A comparison of our RP perovskite LEDs with some highly efficient RP perouskite LEDs from literature.

\begin{tabular}{|c|c|c|c|c|}
\hline RP Perovskite LEDs & EL (nm) & EQE (\%) & Luminance $\left(\mathrm{cdm}^{-2}\right)$ & References \\
\hline $\mathrm{PEA}_{2}\left(\mathrm{FAPbBr}_{3}\right)_{n-1} \mathrm{PbBr}_{4}$ & 530 & 14.36 & 7000 & Yang et al.[33] \\
\hline $\mathrm{NMA}_{2}\left(\mathrm{FAPbI}_{3}\right) \mathrm{PbI}_{4}$ & 760 & 11.7 & - & Wang et al.[31] \\
\hline $\mathrm{PEA}_{2}(\mathrm{MA})_{n-1} \mathrm{~Pb}_{n} \mathrm{Br}_{3 n+1}$ & 520 & 7.4 & 8400 & Quan et al.[29] \\
\hline $\mathrm{Ba}_{2}(\mathrm{MA})_{2} \mathrm{~Pb}_{3} \mathrm{X}_{1} 0$ & $468,523,700$ & $0.01,1.01,2.29$ & $21,2246,214$ & Hi et al.[221] \\
\hline $\mathrm{OA}_{2}(\mathrm{FA})_{n-1} \mathrm{~Pb}_{n} \mathrm{Br}_{3 n+1}$ & 530 & 12.1 & 21,354 & Chin et al.[133] \\
\hline $\mathrm{PBA}_{2}(\mathrm{CsPbBr}-3)_{n-1} \mathrm{PbBr}_{4}$ & 491 & 0.01 & 186 & Cheng et al.[39] \\
\hline $\mathrm{NMA}_{2} \mathrm{Cs}_{n-1} \mathrm{~Pb}_{n} \mathrm{I}_{3 n+1}$ & 680 & 7.3 & 732 & Chang et al. [224] \\
\hline $\mathrm{NF}_{1}-x \mathrm{C}_{x} \mathrm{PI}_{7}$ & 783 & 7.3 & - & Yang et al.[228] \\
\hline $\mathrm{PBA}_{2}(\mathrm{CsPbBr})_{n-1} \mathrm{PbBr}_{4}$ & 514,683 & $10.4,7.3$ & $14000,-$ & Si et al.[30] \\
\hline $\mathrm{PEA}_{2} \mathrm{Cs}_{n-1} \mathrm{~Pb}_{n} \mathrm{Br}_{3 n+1}$ & 501 & 4.5 & 3259 & Shang et al.:[28] \\
\hline $\mathrm{EA}_{2}(\mathrm{MA})_{n-1} \mathrm{~Pb}_{n} \mathrm{Br}_{3 n+1}$ & 485 & 2.6 & 200 & Wang et al.[229] \\
\hline $\mathbf{B} \mathbf{A}_{2} \mathbf{C s}_{n-1} \mathbf{P} \mathbf{b}_{n} \mathbf{X}_{3 n+1}$ & $\begin{array}{l}465,487,505 \\
514,615-681\end{array}$ & $\begin{array}{l}2.4,6.2,10.1 \text {, } \\
7.2,0.08-0.41\end{array}$ & $\begin{array}{c}962,3339,8738 \\
1124,6-313\end{array}$ & Our work \\
\hline
\end{tabular}

\subsubsection{J-V characteristic}

Figure 4.12 shows the current density vs. voltage (J-V) curve of rpp-LEDs. A Keithley 236 source measurement unit was used to conduct the electrical measurements. The JV curve shows a standard diode behaviour. It can be seen that the turn on voltage in most of the devices is around 4.0-5.0 V, where the curve starts to rise in linear axis JV curve. However, device Y546 showed the turnon voltage $\approx 8.0 \mathrm{~V}$. The current density in red devices was found to be higher than blue devices. However, B465 showed higher current density than 0615 and Y546. Overall, all of the devices showed the current in the order of milliamp. Thus, The turn-on voltages in JV curve are consistent with the observed turn-on voltages during the device operation. Power consumption values for these LEDs were also calculated at peak EQE and described in the Table 4.4. Power consumption values for Perovskite LEDs are in the range of 7 to $25 \mathrm{~mW}$ for all devices except Y546, which showed very high value of $152 \mathrm{~mW}$ due to poor performance. 

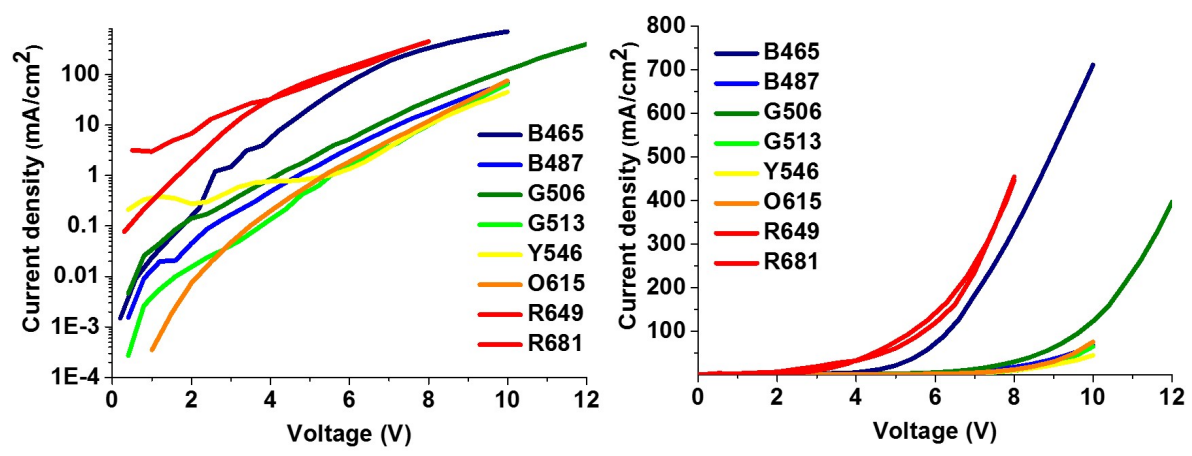

Figure 4.12: Current density vs. voltage curve of RP perovskite LEDs on semi-log and linear axis.

Table 4.4: Power consumption in RP perovskite LEDs at their peak EQE.

\begin{tabular}{|c|c|c|c|c|}
\hline Device Name & $\begin{array}{c}\text { Power Consumption, } \\
\text { at EQE }_{\text {max }}(\mathbf{m W})\end{array}$ & EQE (\%) & $\begin{array}{c}\text { Luminance } \\
\left(\mathbf{c d m}^{-2}\right)\end{array}$ & $\begin{array}{c}\text { Luminous Efficacy } \\
(\mathbf{l m} / \mathbf{W})\end{array}$ \\
\hline B465 & 25.2 & 2.45 & 962 & 0.72 \\
$\mathbf{B 4 8 7}$ & 22.5 & 6.23 & 3339 & 2.80 \\
$\mathbf{G 5 0 6}$ & 7.35 & 10.10 & 3810 & 9.80 \\
$\mathbf{G 5 1 3}$ & 26.4 & 7.22 & 11224 & 8.02 \\
Y546 & 152 & 0.63 & 1066 & 0.13 \\
$\mathbf{O 6 1 5}$ & 25.0 & 0.41 & 313 & 0.26 \\
$\mathbf{R 6 4 9}$ & 9.6 & 0.13 & 18 & 0.03 \\
$\mathbf{R 6 8 1}$ & 15.6 & 0.08 & 5 & 0.007 \\
\hline
\end{tabular}

\subsubsection{Ion Migration}

Similar to other mixed halide perovskites, RP perovskites also exhibit ion migration, although to a relatively lesser degree [20]. Figure 4.13 shows the EL peak position over different voltages for all of the rpp-LEDs. The peak position at each voltage is labelled on its respective graphs. All the peaks eventually moved from their initial positions towards higher wavelengths in mixed halide red de- 
a) $\mathrm{R} 681$

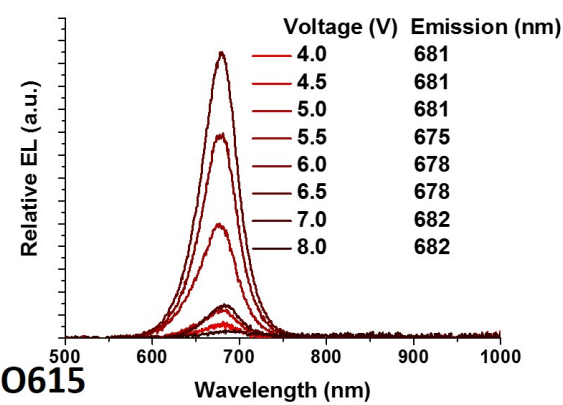

b) R649
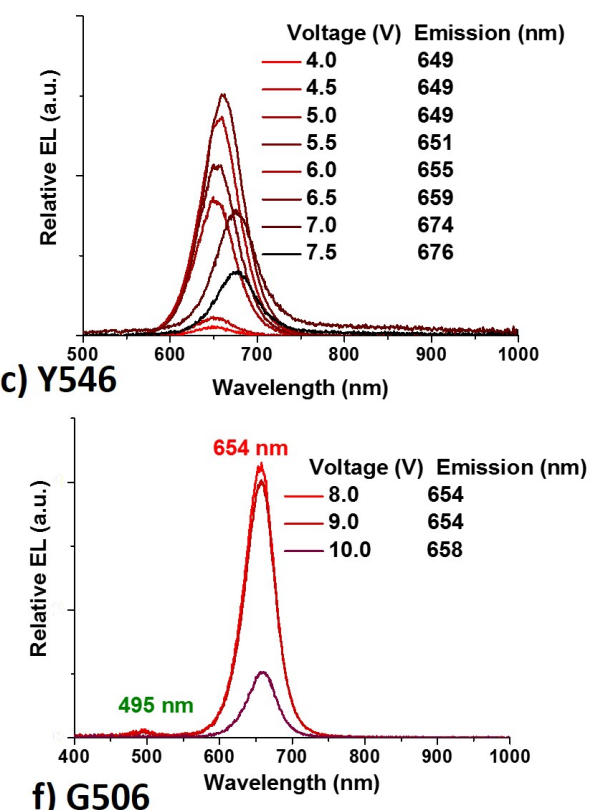

e) G513
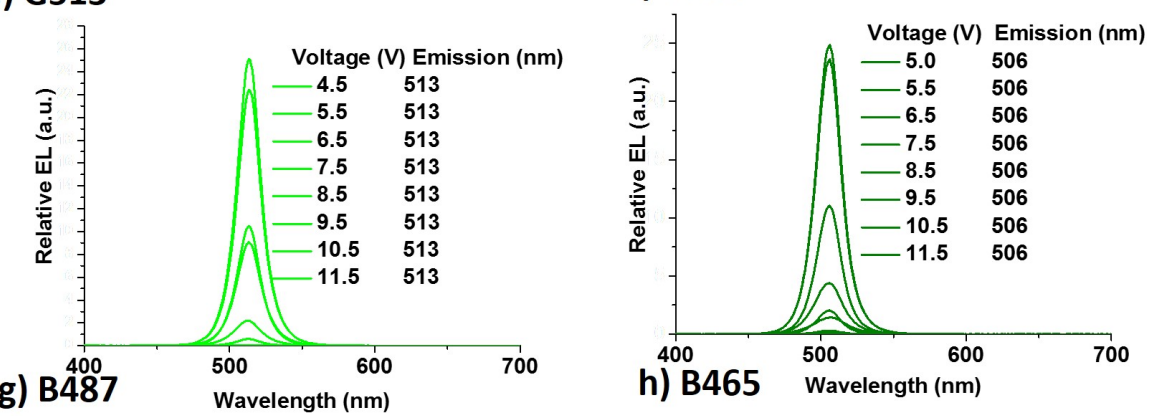

h) B465

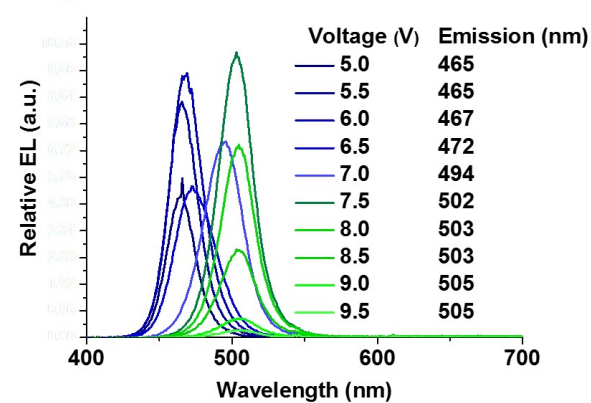

Figure 4.13: EL peak position over different voltage in LEDs for a) R681, b) R649, c) 0615, d) Y546 e) G513, f) G506, g) B487, and h) B465. All the peaks eventually move from their initial positions towards higher wavelengths at high voltage in mixed halide devices. 
vices. The peak shift in Y546 was so fast that the initial peak showed emission at $654 \mathrm{~nm}$. This effect has been seen in perovskite materials due to ion migration, in particular in mixed $\mathrm{Br} / \mathrm{I}$ devices [20]. However, the extent and rate of peak movement in blue devices (B465 and B487) was very slow even at high voltage $(>9.0 \mathrm{~V})$. The EL position in green devices (pure $\mathrm{Br}$ ) was found to be constant over voltage, as it was expected that the ion migration should not occur in pure halide materials.

The similar behaviour has been seen in Chapter 3 as well, where the mixed halide devices showed ion migration. However, the rate of ion migration here was found to be relatively slower due to the RP perovskite structure [86]. As can be seen that the rate of ion migration increases with the increase in higher bromide content orange-red devices. R649 was found to be relatively stable than rest of the mixed halide red LEDs, which is consistent with the previously reported perovskite LEDs [20]. The blue LEDs (B465-B487) were found to be relatively more stable than the red devices even at voltages higher than $9.0 \mathrm{~V}$. However, the emission peak was appeared to be slightly shifted at voltage more than 9.0 V (Figure 4.14). Devices with pure bromide content showed no ion migration and the EL was stable at their initial position even up to $12.0 \mathrm{~V}$, where the device tends to break down. Thus, the ion migration in these devices is yet to be completely resolved. Devices with pure halide perovskite showed no peak shift, but it is not possible to tune the emission spectra in the entire visible region just using the pure halide perovskite materials $(\mathrm{Cl}$, br, or I).

\subsubsection{Stability Test}

For testing the stability in these devices, the LED (B506), emitting at $505 \mathrm{~nm}$, was operated at $7.0 \mathrm{~V}$ and $\approx 26 \mathrm{~mA} / \mathrm{cm}^{2}$ current density. This device ran for $\approx 75 \mathrm{~min}$ in the ambient condition without packaging. The EL, recorded at different time intervals, was found to be at the same position. Figure 4.14 shows the relative EL at different time intervals along with changes in the EQE and 

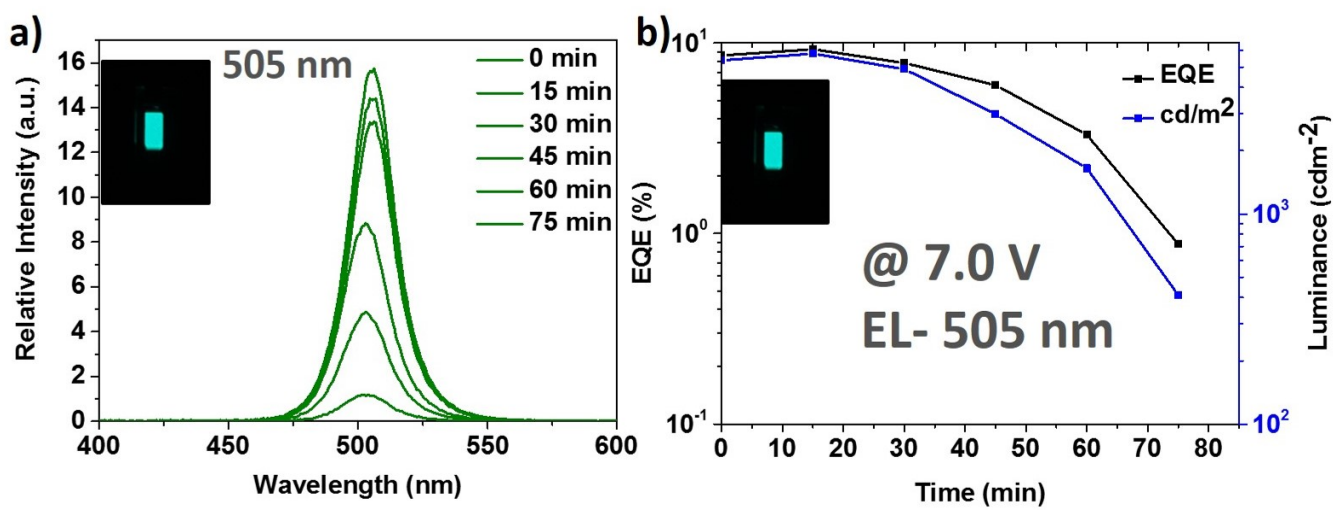

Figure 4.14: a) A chart of the relative intensity of the EL spectra for B505 over a period of 75 min at a bias of $7.0 \mathrm{~V}$ and $\approx 26 \mathrm{~mA} / \mathrm{cm}^{2}$ current density. b) A chart of the EQE, luminance with respect to the time. The unpackaged device ran about 75 min in ambient condition.

the luminance over time. The maximum EQE, for this device, was recorded to be $9.27 \%$ with the luminance of $5782 \mathrm{cdm}^{-2}$. During operation, the EQE remained above $75 \%$ of its original value for first $45 \mathrm{~min}$ of operation. However, the device was unpackaged in ambient conditions, and the organic layers are also sensitive to moisture and air, this suggests that the stability and efficiency of these perovskite LEDs improved significantly using quasi-2D structure than using perovskite NCs (Chapter 3), where the device could ran only about $35 \mathrm{~min}$ at very low EQE of $0.13 \%$ [20]

\subsection{Conclusion}

We have shown solution processed in-situ growth of a colour tuneable RP perovskite materials with the formula of $\mathrm{BA}_{2} \mathrm{Cs}_{n-1} \mathrm{~Pb}_{n}(\mathrm{Br} / \mathrm{Y})_{3 n+1}[\mathrm{y}=\mathrm{Cl}$, I], where the butyammonium was used as a separating layer between the inorganic octahedra. The number of $\mathrm{CsPbBr}_{3}$ monolayers (n) in these $2 \mathrm{D}$ crystals was optimised by changing the butylammonium concentration, which resulted in tuneable optical properties up to some extent. The number of planes in the $2 \mathrm{D}$ 


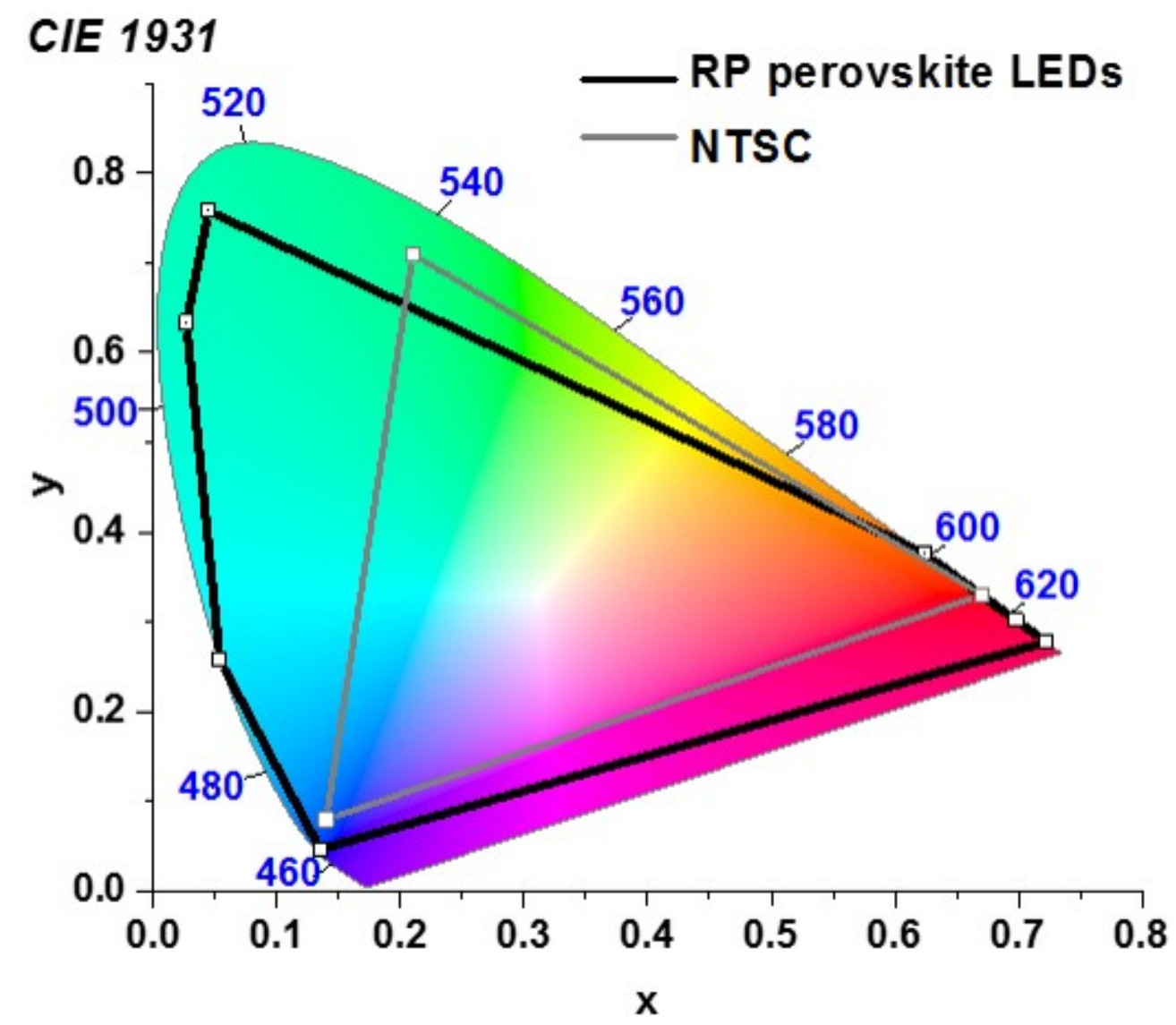

Figure 4.15: The CIE chromaticity coordinate diagram for the CsPbX $\mathrm{RP}$ perovskite LEDs at their turn-on voltages (4.0-5.0 V). The colour triangle from the RP perovskite LEDs (black) is compared with the NTSC colour triangle (gray).

nanosheets of $\mathrm{CsPBBr}_{3}$ were found to be around $\approx 24 \pm 7$ for optimal performance at $505 \mathrm{~nm}$. This balances the thin emission linewidth and high QY of thin nanosheets with the superior conduction properties of thicker materials. For the first time, the emission in caesium based RP perovskite was tuned by halogen mixing.

Furthermore, these RP perovskites were used in the fabrication of highly efficient LEDs emitting in the entire visible spectral region from 465 to $680 \mathrm{~nm}$. We have also shown maximum EQEs of $2.4 \%$ and $6.2 \%$ in the blue spectral region, 
which outperformed the current literature values for any blue perovskite LEDs. The tuning in the green emission spectra is shown by varying the BA amount in pure $\mathrm{CsPBr}_{3}$ and these devices have shown a high maximum EQE of 10.1 $\%$, which is competent with the literature values of some highly efficient rppLEDs [28-30]. While the ion migration issue is yet to be completely resolved for mixed Br/I perovskites, red LEDs were also achieved with this material set. The CIE diagram of all the investigated LEDs showed the colour tuneability across the entire visible spectrum. The thin linewidth of emission at $465 \mathrm{~nm}, 518 \mathrm{~nm}$ and $640 \mathrm{~nm}$ gives points at the far edges of the diagram in National Television Standards Committee (NTSC) (Figure 4.15). Compared with the NTSC colour triangle (gray), the RGB colour triangle (black) of the RP perovskite LEDs shown here is $30 \%$ larger [230]. This proves that we have achieved a wide range of colour tuneability in these LEDs. Additionally, these results show that mixed 2D/3D materials can be tuned for very high performance in the critical blue and green spectral regions. 


\section{Chapter 5}

\section{Shape-, Size-, and}

\section{Composition-Controlled Thallium}

Lead Halide Nanowires and

Nanocrystals with Tunable Band

\section{Gaps}

Parts of this chapter have been published in the following article:

Vashishtha P, Metin DZ, Cryer ME, Chen, K, Hodgkiss J, Gaston N, Halpert JE.

Shape-, Size-, and Composition-Controlled thallium Lead Halide Perovskite Nanowires and Nanocrystals with Tunable Band Gaps. Chemistry of Materials 2018. 


\subsection{Introduction}

Perovskite NCs have proven themselves to be a useful material for both absorptionand emission-based applications such as photodetectors, LEDs, and solar cells $[17,20,231-234]$. In addition to the QD properties of size tunable band gaps, $\mathrm{MAPbX}_{3}$ and $\mathrm{CsPbX}$ have demonstrated extraordinary properties such as facile interparticle anion exchange [214], nearly $100 \%$ PL quantum yield [17, 235], relatively high charge mobility and conductivity, and low temperature particle fusion from 1D and 2D structures [16, 35, 36, 38, 137, 236]. All of these properties have drawn the attention towards investigating materials based on perovskite or perovskite like crystal structures. Some examples are $\mathrm{FAPbX}_{3}$, $\mathrm{MA}_{3} \mathrm{BiX}_{9}, \mathrm{Cs}_{3} \mathrm{BiX}_{9}, \mathrm{CsSnX}_{3}, \mathrm{Cs}_{3} \mathrm{Sb}_{2} \mathrm{Br}_{9}, \mathrm{Cs}_{3} \mathrm{Sb}_{2} \mathrm{Cl}_{9}(\mathrm{X}=\mathrm{Cl}, \mathrm{Br}, \mathrm{I} ; \mathrm{FA}=$ formamidinium; $\mathrm{MA}=$ methylammonium) [19, 22, 23, 126, 237, 238], and other perovskite analogues such as double perovskites, alloys and doped perovskites including $\mathrm{Cs}_{2} \mathrm{AgBiBr}_{6}, \mathrm{Cs}_{2} \mathrm{AgInBr}_{6}, \mathrm{Mn}^{2+}$ - doped caesium lead halide perovskite NCs [24, 239-242]. MAPI is one of the well characterised 3D structured perovskite used for constructing highly efficient photodetector, solar cells and LEDs [15, 243-247]. Therefore, finding novel perovskite and related nanomaterials with useful relevant properties is a major area of research in this field and many researchers have been working to replace $A$ and $B$ groups in the $\mathrm{ABX}_{3}$ perovskite structure with feasible atom such as Bi, In, Ag, Sn, and Cu [22-25].

A potential candidate to replace group $\mathrm{A}$ can be $\mathrm{Rb}$. However, $\mathrm{RbPbX}_{3}$ perovskite crystal was found to be unstable at room temperature and it could only crystallise at perovskite phase above $320^{\circ} \mathrm{C}$ [248]. Another candidate to replace A group is thallium ( $\mathrm{Tl}$ ). Thallium is an abundant monovalent or trivalent cation with crystalline radius of $1.61 \AA$. Tl is also known for its toxicity, such as its carcinogenic and teratogenic effects [249, 250]. Carcinogen substances promote the formation of cancer and teratogen substances disturb the development of fetus. However, Tl has several advantages in semiconductor applications such as X-ray radiation detectors, piezoelectric sensors, lasers, UV-vis 
photodetectors, solar cells, and nonlinear optics applications when doped with rare earth materials [251-257]. Moreover, $\mathrm{TlPbI}_{3}$ was found to be more stable structure than $\mathrm{CsPbI}_{3}$ due to the strong bond between $\mathrm{Tl}$ and I which ultimately results in lower total free energy for the crystal [257].

The bulk thallium lead halide crystals have an indirect band gap of 2.3-4.0 eV. $\mathrm{TlPbI}_{3}$ is a remarkable candidate for X-ray detectors due to its wide band gap $(2.3 \mathrm{eV})$, high atomic number, high photon stopping power, and high density $\left(6.6 \mathrm{~g} / \mathrm{cm}^{3}\right)$ [255]. It is known to have a higher absorption cross section than CdTe [255]. Therefore, the linear attenuation coefficient, which is the intensity of $\mathrm{x}$-rays absorbed on per unit thickness area, for $\mathrm{TlPbI}_{3}$ is higher than other high performing materials for radiation detectors. The higher band gap also prevents the thermal generation of charge carriers, improving the signal to noise ratio at room temperature $[254,255]$. Other stable phase of thallium lead halides such as $\mathrm{Tl}_{3} \mathrm{PbX}_{5}$, a perovskite like material, exists in the form of trivalent cation. $\mathrm{Tl}_{3} \mathrm{PbX}_{5}$ have been used as a mid-infrared and near infrared non linear optics [253, 258, 259] For example, $\mathrm{Er}^{3+}$ or $\mathrm{Pr}^{3+}$ doped $\mathrm{Tl}_{3} \mathrm{PbBr}_{5}$ have been used in mid-IR solid state lasers $[252,256]$. Therefore, NCs of this material can be even more attractive for various applications. $\mathrm{Tl}_{4} \mathrm{PbI}_{6}$ is also one of the crystal phase but only exist above $298{ }^{\circ} \mathrm{C}$ and decomposes to TII and $\mathrm{Tl}_{3} \mathrm{PbI}_{5}$ [260]. Khyzun et al. synthesised high temperature monoclinic crystal structures of $\mathrm{TlPb}_{2} \mathrm{Br}_{5}$ and $\mathrm{TlPb}_{2} \mathrm{Cl}_{5}$ at $400{ }^{\circ} \mathrm{C}$ and $420{ }^{\circ} \mathrm{C}$ respectively [261, 262]. However, In this research, we mainly focused on the synthesis of $\mathrm{TlPbI}_{3}$ and $\mathrm{Tl}_{3} \mathrm{PbX}_{5} \mathrm{NCs}$, which are stable at room temperature.

This chapter includes the synthesis of thallium lead halide NCs and NWs by colloidal route as a novel analogues to inorganic lead halide perovskites [17, 22, 23, 238]. Orthorhombic $\mathrm{TlPbI}_{3}$ perovskite NWs were synthesised with an indirect band gap of $2.30 \mathrm{eV}$. Additionally, an orthorhombic phase of $\mathrm{Tl}_{3} \mathrm{PbI}_{5}$ and $\mathrm{Tl}_{3} \mathrm{PbBr}_{5}$ and a tetragonal phase of $\mathrm{Tl}_{3} \mathrm{PbCl}_{5}$ NCs were synthesised with a tuneable band gap, having a direct transition peak across the UV-blue spectrum, from 280-440 $\mathrm{nm}$. The band gap was tuned by varying the halide mixture 
$\mathrm{Tl}_{3} \mathrm{PbY}_{5-x} \mathrm{Br}_{5}(\mathrm{Y}=\mathrm{Cl}, \mathrm{I})$ in mixed halide concentration and to a lesser degree, by changing the particle size. Size tuning was achieved by changing the reaction conditions in the synthesis of $\mathrm{Tl}_{3} \mathrm{PbBr}_{5}$ NCs. $\mathrm{Tl}_{3} \mathrm{PbI}_{5}$ NCs also showed a weak PL. Furthermore, we demonstrate control over the shape and crystal structure of thallium lead iodide by altering the reaction conditions to produce $\mathrm{TlPbX}_{3}$ perovskite NWs with high aspect ratios and lengths greater than $4 \mu \mathrm{m}$. The chapter also displays an application of $\mathrm{TlPbI}_{3} \mathrm{NWs}$ as a wide band gap photoconductor.

\subsection{Experimental}

This section describes the synthesis of thallium lead halide NWs and NCs. This also includes how the reaction conditions were varried to control the size, shape, and composition of materails.

\subsubsection{Synthesis of $\mathrm{Tl}_{3} \mathrm{PbI}_{5}$ Perovskite NWs}

\subsubsection{Materials}

Oleic acid (90\%), oleylamine (99\%), thallium(I) acetate (99 \%), 1-octadecene (90\%), $\mathrm{PbBr}_{2}$ (99.9 \% trace metal basis), $\mathrm{PbCl}_{2}$ (99.9\% trace metal basis), $\mathrm{PbI}_{2}$ (99.9 \% trace metal basis), hexane (95 \% anhydrous), acetonitrile (99.8 \% anhydrous), and tolune (99.8\% anhydrous) were purchased from Sigma-Aldrich. Oleylamine and oleic were stored in the refrigerator. Anhydrous solvents such as, acetonitrile, hexane, and toluene were stored in a nitrogen glove box. 


\subsubsection{Method}

This is the first report for the synthesis of thallium lead halide NCs. A hot injection colloidal route was used to synthesise these NCs. In the synthesis method, thallium oleate was used as a thallium source; $\mathrm{PbI}_{2}$ was used as a lead and iodide source. Oleylamine and oleic acid were used to dissolve lead iodide in 1-octadecene and also served as a capping agent. The synthesis procedure was divided into two parts; first is the synthesis of precursor, which is thallium oleate and then the synthesis of $\mathrm{TlPbI}_{3} \mathrm{NWs}$

Synthesis of Precursor: Thallium oleate was synthesised by loading $0.46 \mathrm{gm}$ of thallium(I) acetate, $15 \mathrm{~mL}$ of 1-octadecene, and $1.6 \mathrm{~mL}$ of oleic acid into a $50 \mathrm{~mL}$ of 3-neck round bottom flask. The reaction mixture was degassed under vacuum at $120^{\circ} \mathrm{C}$ for $1 \mathrm{~h}$. Then, the temperature was raised to $150{ }^{\circ} \mathrm{C}$ for next 30 min and the reaction was switched to $\mathrm{N}_{2}$ from vacuum. The final pale yellow colour solution was the thallium oleate, which was transferred to a Schlenk tube and stored in $\mathrm{N}_{2}$ glove box until it was required for the synthesis of NCs.

Synthesis of NCs: A $0.38 \mathrm{mmol}$ portion of $\mathrm{PbX}_{2}$ was loaded in to a $50 \mathrm{~mL} 3-$ neck round bottom flask along with $10 \mathrm{~mL}$ of 1-octadecene. The reaction solution was degassed under vacuum at $120{ }^{\circ} \mathrm{C}$ for $1 \mathrm{~h}$. Simultaneously, $1.0 \mathrm{~mL}$ oleylamine and oleic acid were also degassed under the same conditions in two separate vials. After degassing, oleylamine and oleic acid were injected into the reaction flask under $\mathrm{N}_{2}$, and the temperature was raised to $130-175{ }^{\circ} \mathrm{C}$. After this, $1.1 \mathrm{~mL}$ of thallium oleate was quickly injected into the reaction flask. After $45 \mathrm{~s}$ the reaction flask was cooled down using an ice bath.

The reaction rate was found to be similar like $\mathrm{CsPbX}_{3} \mathrm{NCs}$. Therefore, reaction flask was quickly cooled down in order to prevent the agglomeration. The particle size was controlled by reaction temperature and the composition tuning was achieved by mixing the halide precursors in the reaction. Table 5.1 explains 
the amount for each precursor with reaction temperature and time in detail.

Purification of NCs: In order to remove the excess ligands and organics from NCs surface, they should be washed properly by solvent/anti-solvent method. NCs were capped with hydrophobic ligands therefore special precautions needed to be taken while choosing the right anti-solvent. The same purification method was used as explained in Chapter 2 (2.2.1.2).

The growth solution was centrifuged at 10,000 rpm for $10 \mathrm{~min}$, and then the supernatant was discarded. The precipitate was redispersed in $6 \mathrm{~mL}$ of toluene with $3 \mathrm{~mL}$ of acetonitrile as an antisolvent and centrifuged again at 10,000 rpm for $10 \mathrm{~min}$. After discarding the supernatant, the precipitate was dried under vacuum for $5 \mathrm{~min}$ and then redispersed in hexane. This purification process could be repeated for at least two cycles in all applications and as many as four cycles for elemental analysis.

\subsubsection{Synthesis of $\mathrm{TlPbI}_{3} \mathrm{NWs}$}

The synthesis of NWs was also divided into two parts: 1) synthesis of thallium oleate precursor and 2) synthesis of $\mathrm{TlPbI}_{3} \mathrm{NWs}$. The precursor was synthesised by the same method as described in the Section 5.2.2, where thallium(I) acetate, 1-octadecene, and oleic acid were degassed under vacuum followed by heating at $150{ }^{\circ} \mathrm{C}$ under nitrogen. After the synthesis, thallium oleate was transferred in a Schlenk tube under nitrogen and stored in a nitrogen glove box.

A $0.38 \mathrm{mmol}$ of $\mathrm{PbI}_{2}$ was loaded into a $50 \mathrm{~mL}$ 3-neck round bottom flask with $10 \mathrm{~mL}$ of 1-octadecene. The reaction solution was degassed under vacuum at $120{ }^{\circ} \mathrm{C}$ for $1 \mathrm{~h}$. Simultaneously, $0.5 \mathrm{~mL}$ of oleylamine and oleic acid were degassed under vacuum in two separate vials. After degassing, oleylamine and oleic acid were injected into the reaction flask under nitrogen at $120^{\circ} \mathrm{C}$, and the temperature was raised to $170^{\circ} \mathrm{C}$ for hot injection. A $0.9 \mathrm{~mL}$ portion of thallium 
oleate was quickly injected in to the reaction flask, and in $30 \mathrm{~s}$, it was cooled down with the ice bath. $\mathrm{TlPbBr}_{3}$ and $\mathrm{TlPbCl}_{3}$ NWs were not observed during the synthesis. It was found that even the bulk crystals of those have not been reported at atmospheric pressure [263]. Therefore, even at higher temperature, only larger $\mathrm{NCs}$ of $\mathrm{Tl}_{3} \mathrm{PbX}_{5}(\mathrm{X}=\mathrm{Br}, \mathrm{Cl})$ could be observed (Table 5.1).

The purification was similar to the purification thallium lead halide NCs (Section 5.2.2). The growth solution was centrifuged at 10,000 rpm for $10 \mathrm{~min}$. After that, the supernatant was discarded, and the precipitate was redispersed in 6 $\mathrm{mL}$ of toluene (solvent) with $3 \mathrm{~mL}$ of acetonitrile (antisolvent) and centrifuged again at 10,000 rpm for $10 \mathrm{~min}$. After discarding the supernatant, the precipitate was dried under vacuum for $5 \mathrm{~min}$ and then redispersed in $10 \mathrm{~mL}$ of hexane. The purification process was conducted in a nitrogen glove box due to the high toxicity of thallium.

Table 5.1: A table of synthesis conditions for thallium lead halide along with the resultant particle size.

\begin{tabular}{|c|c|c|c|c|c|c|c|}
\hline $\begin{array}{c}\text { Sample } \\
\text { name }\end{array}$ & $\begin{array}{c}\text { lead halide } \\
(\mathbf{m m o l})\end{array}$ & $\begin{array}{c}\text { thallium oleate } \\
(\mathbf{m L})\end{array}$ & $\begin{array}{c}\text { oleic acid } \\
(\mathbf{m L})\end{array}$ & $\begin{array}{c}\text { oleylamine } \\
(\mathbf{m L})\end{array}$ & $\begin{array}{c}\text { Temperature } \\
\left({ }^{\circ} \mathbf{C}\right)\end{array}$ & $\begin{array}{c}\text { Time } \\
(\mathbf{s})\end{array}$ & $\begin{array}{c}\text { particle } \\
\text { size }\end{array}$ \\
\hline $\mathrm{TlPbI}_{3} \mathrm{NWs}$ & 0.38 & 0.9 & 0.5 & 0.5 & 170 & 30 & $45.1 \pm 9.3$ \\
$\mathrm{Tl}_{3} \mathrm{PbI}_{5} \mathrm{NCs}$ & 0.38 & 1.1 & 1.0 & 1.0 & 130 & 45 & $17.2 \pm 3.0$ \\
$\mathrm{Tl}_{3} \mathrm{~Pb}(\mathrm{Br} / \mathrm{I})_{5}$ & 0.38 & 1.1 & 1.0 & 1.0 & 130 & 45 & $15.2 \pm 2.2$ \\
$\mathrm{Tl}_{3} \mathrm{PbBr}_{5}$ & 0.38 & 1.1 & 1.0 & 1.0 & 180 & 45 & $28.0 \pm 2.5$ \\
$\mathrm{Tl}_{3} \mathrm{PbBr}_{5}$ & 0.38 & 1.1 & 1.0 & 1.0 & 160 & 45 & $20.9 \pm 3.0$ \\
$\mathrm{Tl}_{3} \mathrm{PbBr}_{5}$ & 0.38 & 1.1 & 1.0 & 1.0 & 130 & 45 & $16.5 \pm 2.5$ \\
$\mathrm{Tl}_{3} \mathrm{~Pb}(\mathrm{Br} / \mathrm{Cl})_{5}$ & 0.38 & 1.1 & 1.0 & 1.0 & 130 & 45 & $19.2 \pm 1.7$ \\
$\mathrm{Tl}_{3} \mathrm{PbCl}_{5}$ & 0.38 & 1.1 & 1.0 & 1.0 & 165 & 45 & $20.5 \pm 2.7$ \\
\hline
\end{tabular}

\subsubsection{Inductively Coupled Plasma Mass Spectrometry (ICP-MS)}

ICP-MS was carried out by Dr Bruce Charlier at the School of Geography, Environmental, and Earth Sciences, Victoria University of Wellington. These measurements were carried out to measure the elemental ratio between thallium 
and lead. Sample were purified three times by solvent/anti-solvent method and then dissolved in nitric acid. Stock thallium and lead ICP-MS standards certified at $1000 \mathrm{ppm}$ were diluted in two stages using ultra-pure $1 \% \mathrm{HNO}_{3}$ to produce a series of gravimetric instrument calibration standards at 1, 2, 5 and $10 \mathrm{ppb}$. Tl-Pb samples were supplied as solutions and diluted to similar levels as the standards also using $1 \% \mathrm{HNO}_{3} . \mathrm{Pb}$ and $\mathrm{Tl}$ were measured in the calibration standards, blank solutions (HNO3 used for all dilutions) and samples using a Thermo Element II high resolution ICP-MS. Measured counts for Tl (atomic mass 205) and $\mathrm{Pb}$ (atomic mass 208) in the diluent acid were subtracted from those for the samples, then compared with the calibration standards in order to calculate the concentrations for $\mathrm{Tl}$ and $\mathrm{Pb}$ in the diluted samples. The final concentration in the supplied solutions was then calculated by applying a dilution factor determined through gravimetry from the sample preparation.

\subsubsection{Electrical Measurements}

Electrical measurements on NWs were carried out by Matthew Cryer under the supervision of Prof Jonathan E. Halpert at the School of Chemical and Physical Sciences, Victoria University of Wellington. The devices used for electrical measurements were $\mathrm{Si} / \mathrm{SiO}_{2}$ wafers with $300 \mathrm{~nm}$ oxide layers. The first step in fabrication was surface treatment, [(3-aminopropyl) trimethoxysilane in dry toluene for $30 \mathrm{~min}$ ] to deposit a silane self-assembled monolayer (SAM). $\mathrm{Cr} / \mathrm{Au}$ $(5 / 50 \mathrm{~nm}$ ) interdigitated electrode (IDE) (34 fingers per side, $2 \mathrm{~mm}$ long with $20 \mu \mathrm{m}$ spacing; device area is $5 \times 10^{-6} \mathrm{~m}^{2}$ networks were deposited by a 1step process using photolithography. The NWs were spin-cast from hexane onto the IDEs at $1000 \mathrm{rpm}$ for $40 \mathrm{~s}$. After each layer, the NWs were quickly rinsed in ethanol and then dried with nitrogen. Before measurement, the base and edges were cleaned with chloroform. Electrical measurements were conducted with a Keithley SCS-4200 parameter analyser. The device was placed on a temperature-controlled mount in a Janis VNF-100 liquid nitrogen cryo- 
stat and connected to the SCS-4200 instrument. Measurements were then conducted in the dark, and when illuminated with a Thorlabs $405 \mathrm{~nm}$ laser diode $\left(6.4 \mathrm{~mW} / \mathrm{cm}^{2}\right.$ ), the optical path consisted of $5 \mathrm{~cm}$ in air and $2 \mathrm{Al}_{2} \mathrm{O}_{3}$ windows (85 $\%$ transmission at $405 \mathrm{~nm}$ ). In addition, measurements were carried out, when the sample was illuminated with a microscope light at different intensities.

\subsection{Results and Discussion}

NCs were characterised by spectroscopic and microscopic techniques. TEM is a promising tool to observe the morphology of NCs. XRD measurements were conducted to confirm the crystal structure of the NCs. Crystallographic analysis was conducted with the help of Dr Anna Henning. A theoretical modeling was also performed by Dani Z. Metin at University of Auckland to model the crystals and XRD spectra. UV-Vis measurements were carried out to observe the optical properties such as band gap of the material. PL measurements were carried out by Dr. Kai Chen at Victoria University of Wellington to observe the carrier lifetime and emission properties in these materials.

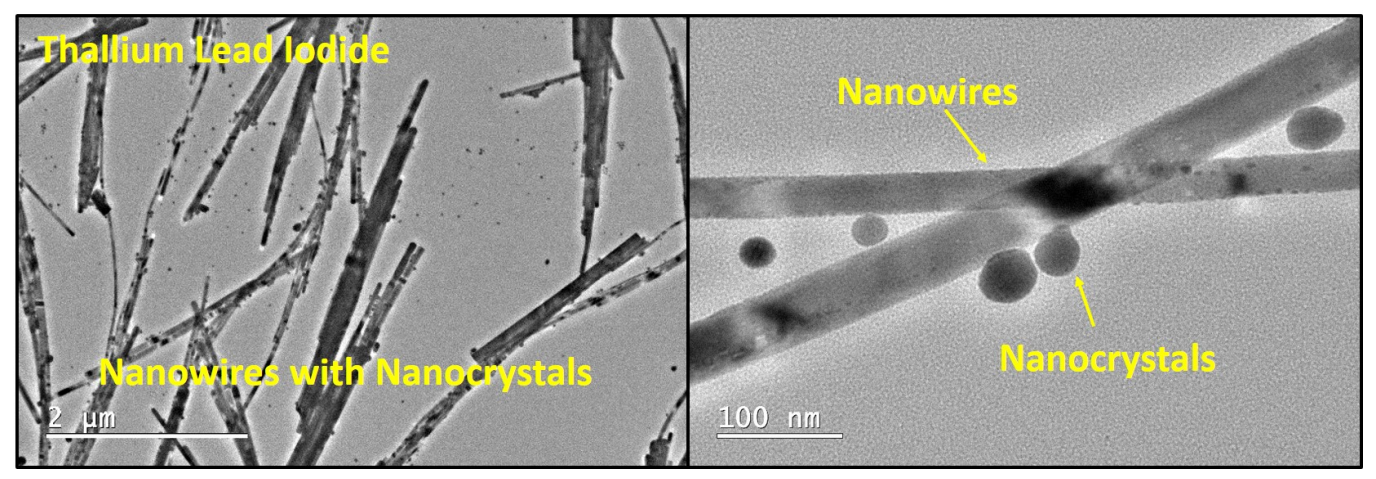

Figure 5.1: TEM micrographs of thallium lead iodide NWs. The reaction results the formation of NWs along with some NCs, which is not desirable for semiconducting application. The TEM samples were prepared by drop-casting hexane NWs solution on a $\mathrm{Cu}-200$ mesh on formvar grid. 


\subsubsection{Morphological Characterisation on Thallium Lead Iodide}

In the first attempt of thallium lead iodide synthesis, we were able to achieve the mixture of NCs and NWs (Figure 5.1). In this reaction $2.0 \mathrm{~mL}$ of oleylamine and oleic acid were used at $160{ }^{\circ} \mathrm{C}$ for the same amount of other precursors as explained in Table 5.1. The mixture of two different shapes is not ideal for semiconducting applications, and therefore, control over reaction condition is required to achieve NCs and NWs separately.

\subsubsection{1 $\mathrm{TlPbI}_{3} \mathrm{NWs}$}

The reaction conditions were optimised by varying the amount of the ligand and the reaction temperature. Pure NWs were obtained by decreasing the amount of ligands to $0.5 \mathrm{~mL}$ and increasing the temperature to $170{ }^{\circ} \mathrm{C}$. This was possibly favorable to get the anisotropic growth for synthesis of NWs. Figure 5.2a shows the TEM micrograph of optimised NWs, where we were able to observe mostly NWs. The NWs size was measured by ImageJ and then the histogram was created, which is shown on top of the wider angle TEM micorgraph of NWs. NWs were found to be longer than $4 \mu \mathrm{m}$ with $\approx 45 \mathrm{~nm}$ mean width.

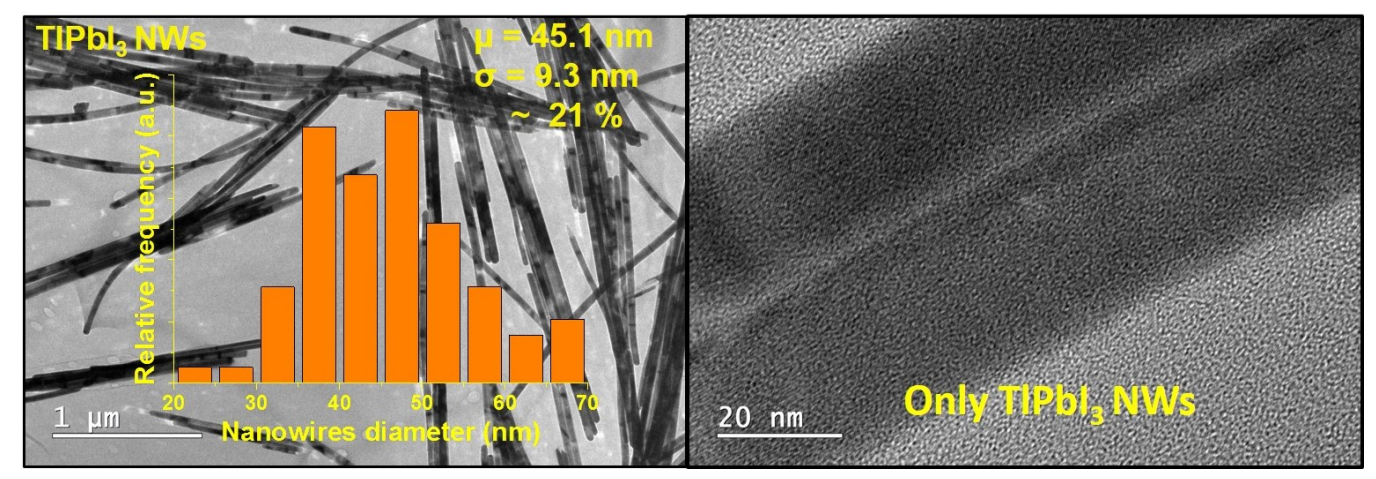

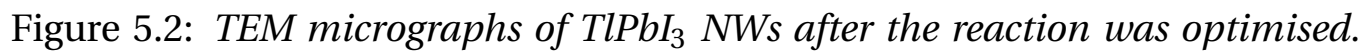
Wide angle image (left) shows there is no NCs and the reaction results the formation of pure NWs. A histogram on top of wider angle TEM image displays the mean width of $45.1 \pm 9.3 \mathrm{~nm}$. The length of the particles is greater than $4 \mu \mathrm{m}$. 


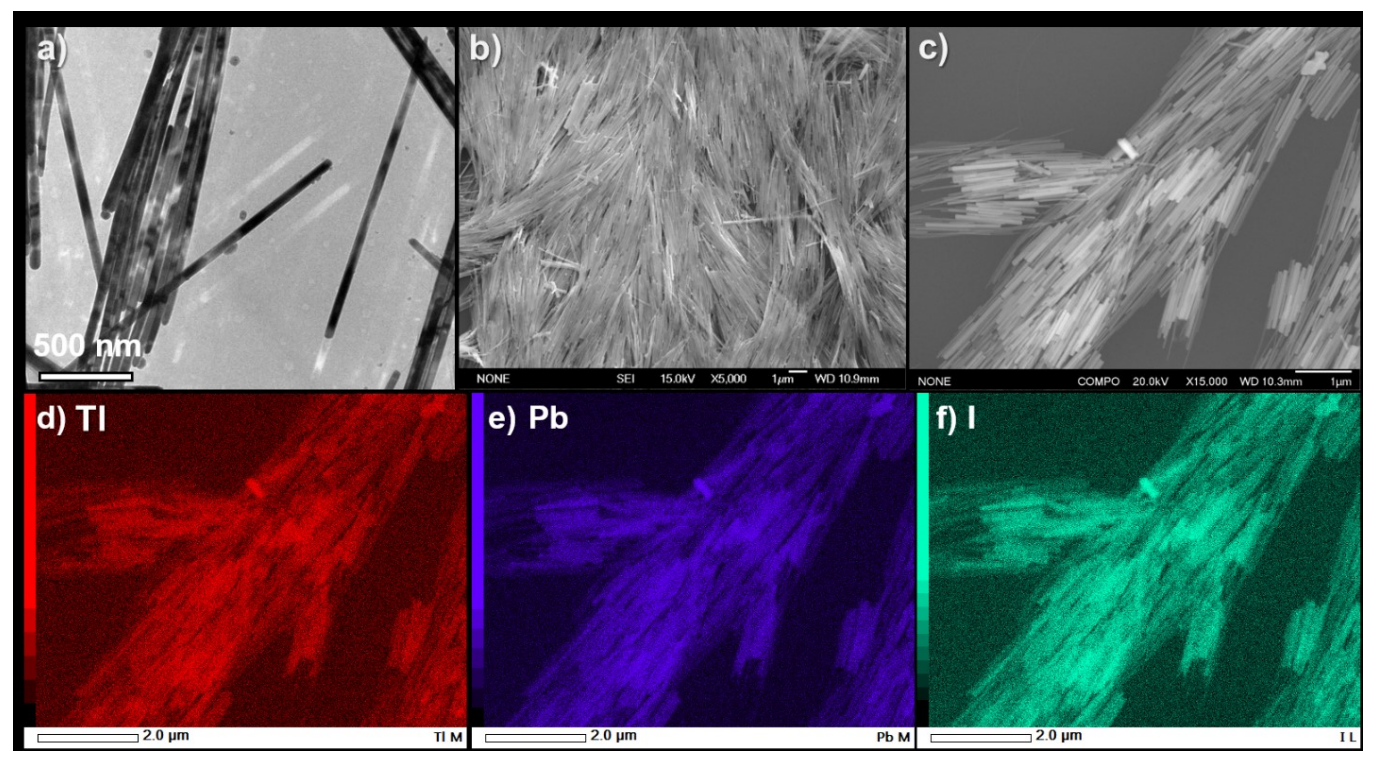

Figure 5.3: a) TEM image of $\mathrm{TlPbI}_{3} \mathrm{NWs}$, b) SEM image of $\mathrm{TlPbI}_{3} \mathrm{NWs}$, c) SEM image of the NWs for EDS mapping, and d), e), f) SEM maps for $\mathrm{Tl}, \mathrm{Pb}$ and $\mathrm{I}$. An ICP-MS analysis confirms the presence of Tl and Pb in a 1:1 stoichiometric ratio. Two terminal device (Section 5.2.5) were used for SEM imaging. Copyright, American Chemical Society 2018.

NWs were further examined by SEM EDS Mapping (Figure 5.3) to observe each element from $\mathrm{TlPbI}_{3}$. Two terminal device, which was fabricated to carry the electrical characterisation (Section 5.2.5), was used for the SEM analysis. Tl and $\mathrm{Pb}$ have the EDS peak position at the same place, therefore it was not possible to conduct the exact quantitative analysis. However, EDS mapping (Figure 5.3) showed that NWs contain $\mathrm{Tl}, \mathrm{Pb}$, and I. ICP-MS measurements were conducted at the Department of Geography by Dr Bruce Charlier (Section 5.2.4) to quantify the ratio between $\mathrm{Tl}$ and $\mathrm{Pb}$ which was recorded to be 0.9814 . Thus, the elemental analysis confirms the formation of $\mathrm{TlPbI}_{3} \mathrm{NWs}$.

While, the elemental analysis confirmed the formation of $\mathrm{TlPbI}_{3}$. The crystal structure also needed to be analysed. Therefore, XRD studies were conducted using a PANalytical X-Ray diffractometer. The NWs samples for XRD, were prepared by drop-casting the concentrated NWs solution (in hexane) on to a clean 


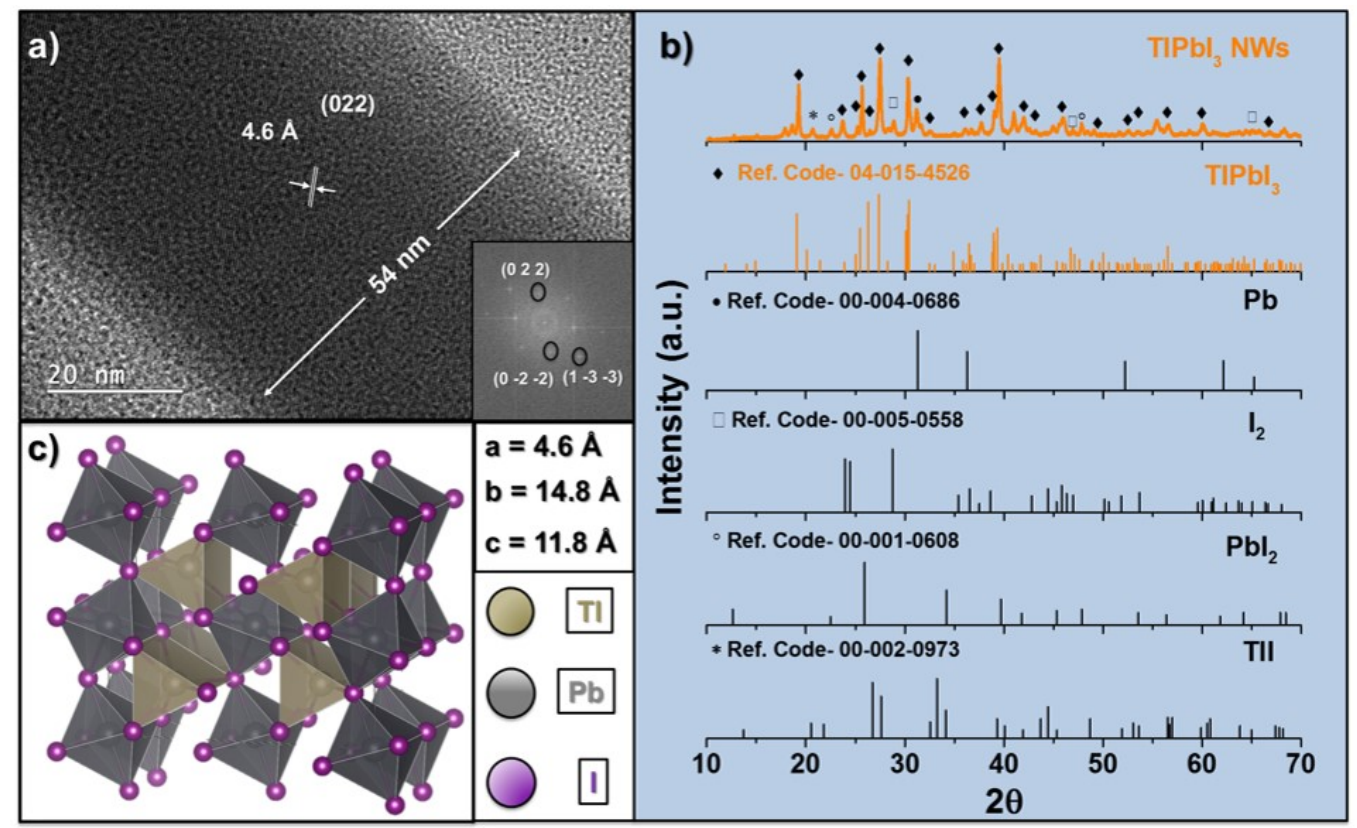

Figure 5.4: (a) High-resolution TEM micrograph of $\mathrm{TlPbI}_{3} \mathrm{NWs}$ showing the lattice spacing and FFT planes. (b) XRD analysis of NWs and comparison with the standard of orthorhombic $\mathrm{TlPbI}_{3}$ crystal structure. XRD patterns were recorded by a PANalytical X-Ray diffractometer using $\mathrm{Cu}$-Karadiation, with an operating voltage of $45 \mathrm{kV}$ and current of $40 \mathrm{~mA}$. The standard spectra of TlPbI $\mathrm{I}_{3}$ was taken from PANalytical X'Pert HighScore Plus with the reference code 04-015-4526. (c) Orthorhombic crystal structure of $\mathrm{TlPbI}_{3}$. Copyright, American Chemical Society 2018.

glass slide. Figure $5.4 \mathrm{c}$ shows the $\mathrm{XRD}$ spectrum of $\mathrm{TlPbI}_{3} \mathrm{NWs}$, which suggests the formation of orthorhombic $\mathrm{TlPbI}_{3}$ crystal structure. The standard spectra are taken from X'Pert Highscore Plus software. The spectra is also compared with some possible side products, such as TlI, $\mathrm{PbI}_{2}, \mathrm{~Pb}$ and $\mathrm{I}_{2}$. Each peak is labelled with its possible corresponding standard peak. It can be seen that most of the peaks are matched with the standard spectra of $\mathrm{TlPbI}_{3}$ with reference code 04-015-4526. The high resolution TEM image on single NWs is shown in Figure 5.4a, which was used to create a fast Fourier transform (FFT) using imageJ software. The orthorhombic crystal structure of $\mathrm{TlPbI}_{3}$ (Figure 5.4c) was 
modeled from the same X'Pert HighScore Plus software data file (04-015-4526). CaRIne software was used to draw the orthorhombic crystal structure with a given lattice parameter to create the fast Fourier transform (FFT). The FFT from the TEM image and the FFT from the crystal structure drawn by CaRIne were compared. The matching of lattice planes in fast Fourier transform (FFT) with standard XRD crystal structure of $\mathrm{TlPbI}_{3}$ also supports the XRD analysis (Figure $5.4 \mathrm{~b}$ ) and suggests the formation of $\mathrm{TlPbI}_{3}$ perovskite material. Thus, the crystal structure and elemental analysis concluded the formation of $\mathrm{TlPbI}_{3} \mathrm{NWs}$. The orthorhombic crystal has the $\mathrm{Cmcm}$ space group with lattice parameters 4.6 , 14.8, and 11.8 A. In a typical sample the mean width was found to be $45.1 \pm 9.3$ $\mathrm{nm}$ with the wires growing along the 133 plane and minimal formation of side products. The stability of $\mathrm{TlPbX}_{3}$ orthorhombic perovskite crystal structure was also calculated theoretically using Goldschmidt tolerance factors, which is the indicator for distortion and stability of perovskite crystal [264]. Equation 5.1 was used to calculate the tolerance factor, where $r_{A}$ is the radius of cation $\mathrm{A}$, which is $\mathrm{Tl}$ in our case; $\mathrm{r}_{B}$ is the radius of cation $\mathrm{B}$, which is $\mathrm{Pb}$; and $\mathrm{r}_{X}$ is the radius of anion $\mathrm{X}$, which is $\mathrm{I}, \mathrm{Br}$, or $\mathrm{Cl}$. All the values for ionic radii were taken from CRC Handbook of Chemistry and Physics [249].

$$
t=\frac{R_{A}+R_{B}}{\sqrt{2} r_{B}+r_{X}}
$$

The Goldschmidt tolerance factors were calculated to be $0.772,0.778$, and 0.780 for I, $\mathrm{Br}$, and $\mathrm{Cl}$, respectively, are all in the range of stable orthorhombic perovskite structures [265]. However, bulk $\mathrm{ABX}_{3}$ perovskite of thallium lead chloride and bromide have not been reported at atmospheric pressure [263]. We were also not able to synthesise NCs or NWs of $\mathrm{TlPbBr} 3$ and $\mathrm{TlPbCl}_{3}$ phase, which is worthy of future investigation. Possibly, a high pressure synthesis setup will be required to synthesise these crystals. However, further investigations could be the part of future research. 


\subsubsection{2 $\mathrm{Tl}_{3} \mathrm{PbI}_{5} \mathrm{NCs}$}

$\mathrm{Tl}_{3} \mathrm{PbI}_{5}$ structure expects to form at lower temperature and is relatively a stable structure than $\mathrm{TlPbI}_{3}[251,266]$. Therefore, by changing the reaction temperature and ligand concentration (Table 5.1), it was possible to change the composition and shape of NWs. We were able to synthesise $\mathrm{Tl}_{3} \mathrm{PbI}_{5} \mathrm{NCs}$ with faceted spheroidal shape. The particle size was measured by creating a histogram of each TEM image using ImageJ (Figure 5.5d). The size for $\mathrm{Tl}_{3} \mathrm{PbI}_{5}$ was calculated to be $17.2 \pm$ 3.0. Crystallographic structures of the orthorhombic $\mathrm{Tl}_{3} \mathrm{PbI}_{5}$ are based on the work of Keller et al. [267, 268] but were fully optimised using

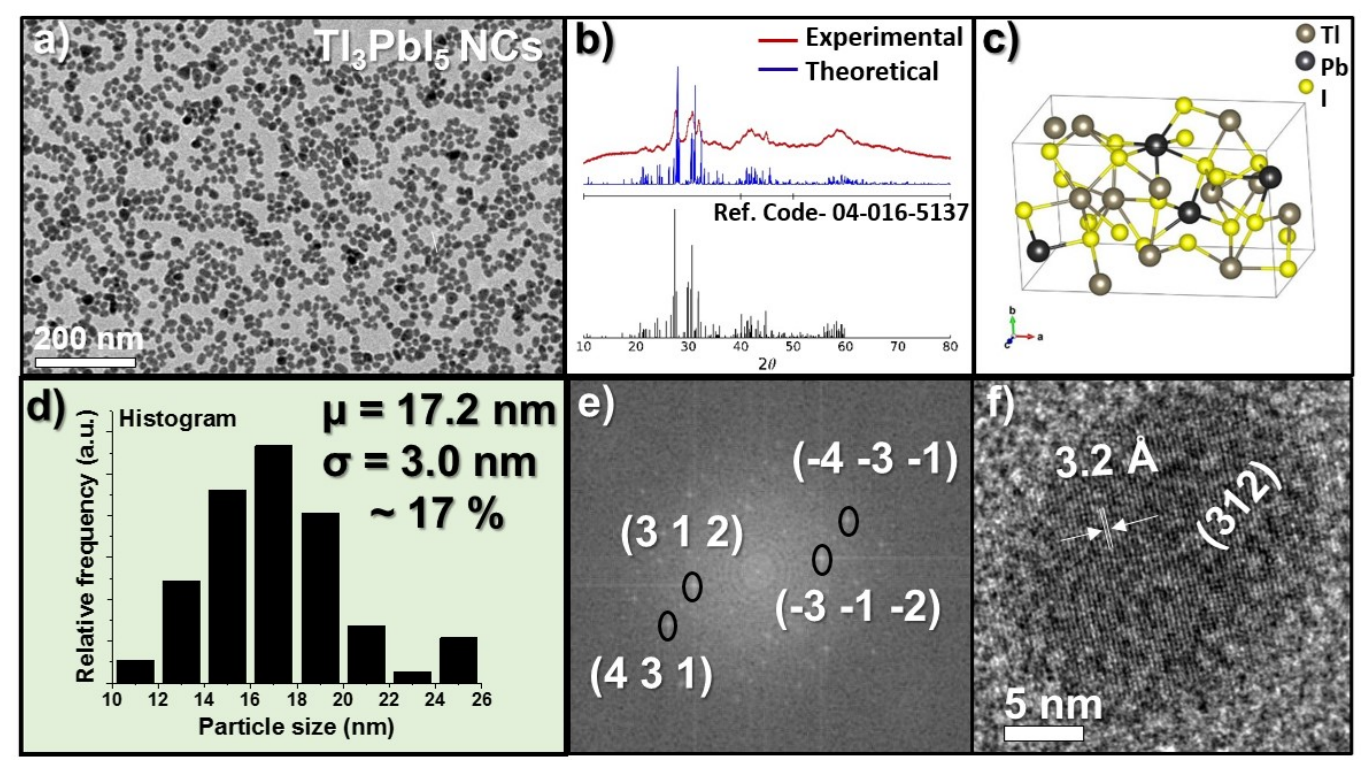

Figure 5.5: a) TEM micrograph of $\left.\mathrm{Tl}_{3} \mathrm{PbI}_{5} \mathrm{NCs}, b\right) \mathrm{XRD}$ spectrum of the same NCs, matched to index spectrum (X'pert HighScore Plus), as well as spectrum theoretically modelled in VESTA, c) the respective periodic crystal structures, 47-48 which have been optimised in VASP, d) Histogram of TEM micrograph produced using ImageJ shows the particle size of $17.2 \pm 3.0 \mathrm{~nm}$, and f) High resolution TEM micrographs of $\mathrm{Tl}_{3} \mathrm{PbI}_{5}$ NCs NCs along with the e) FFT of crystal planes. CaRIne was used to make the crystal structure and the FFT was compared with the FFT of high resolution TEM image $(f)$. 
first-principles calculations by Dani Z. Metin. The details of theoretical modeling, performed by Dani Z. Metin, can be found in our published report [8]. That crystal structure was then used to extract theoretical XRD spectra shown in Figure 5.5. Figure 5.5b shows the XRD spectrum of $\mathrm{Tl}_{3} \mathrm{PbI}_{5} \mathrm{NCs}$ measured by PANalytical X-ray diffractometer. The XRD spectrum for $\mathrm{Tl}_{3} \mathrm{PbI}_{5}$, was matched to the index spectra using Panalytical X'pert Highscore Plus software data file 04-016-5137. XRD spectrum was also matched with the theoretically modeled spectra in VESTA. The XRD peaks were also found to be broadened compared to the bulk index peaks according to the Scherrer equation 1.39. Lattice fringes in NCs are clearly visible in a high resolution TEM image (Figure 5.5f). The visible lattice fringes of $\mathrm{Tl}_{3} \mathrm{PbI}_{5}$ were matched to the (312) crystal plane of the lattice structures with space group P212121 (Figure 5.5a). Lattice planes were further verified by first creating an orthorhombic crystal structure with given lattice parameter using CaRIne software and then comparing the FFT of this crystal structure with the FFT recorded by TEM. Figure 5.5e shows the FFT from high resolution TEM image $\mathrm{Tl}_{3} \mathrm{PbI}_{5} \mathrm{NCs}$. The FFT from the TEM image and the FFT from the crystal structure drawn by CaRIne were compared. The matching of lattice planes in fast Fourier transform (FFT) with standard crystal structure of $\mathrm{Tl}_{3} \mathrm{PbI}_{5}$ obtained by XRD data file also supports the XRD analysis (Figure $5.5 \mathrm{e}$ ). All of these results confirm the formation of orthorhombic crystal structure for $\mathrm{Tl}_{3} \mathrm{PbI}_{5} \mathrm{NCs}$.

\subsubsection{3 $\mathrm{Tl}_{3} \mathrm{PbBr}_{5}$ and $\mathrm{Tl}_{3} \mathrm{PbCl}_{5} \mathrm{NCs}$}

A similar reaction was also able to produce bromide and chloride of $\mathrm{Tl}_{3} \mathrm{PbX}_{5}$ $\mathrm{NCs}$ by changing the halide precursor from $\mathrm{PbI}_{2}$ to $\mathrm{PbBr}_{2}$ and $\mathrm{PbCl}_{2}$ (Table 5.1). Halide composition tuning has been observed in other perovskite materials as well, which allows us to tune the optical properties of the materials [17]. Figure 5.7 shows the TEM images of $\mathrm{Tl}_{3} \mathrm{PbBr}_{5}$ and $\mathrm{Tl}_{3} \mathrm{PbCl}_{5}$ NCs with a faceted spheroidal shape. Crystallographic structures of the orthorhombic $\mathrm{Tl}_{3} \mathrm{PbBr}_{5}$ and tetragonal $\mathrm{Tl}_{3} \mathrm{PbCl}_{5}$ are based on the work of Keller et al. [267, 268], but 


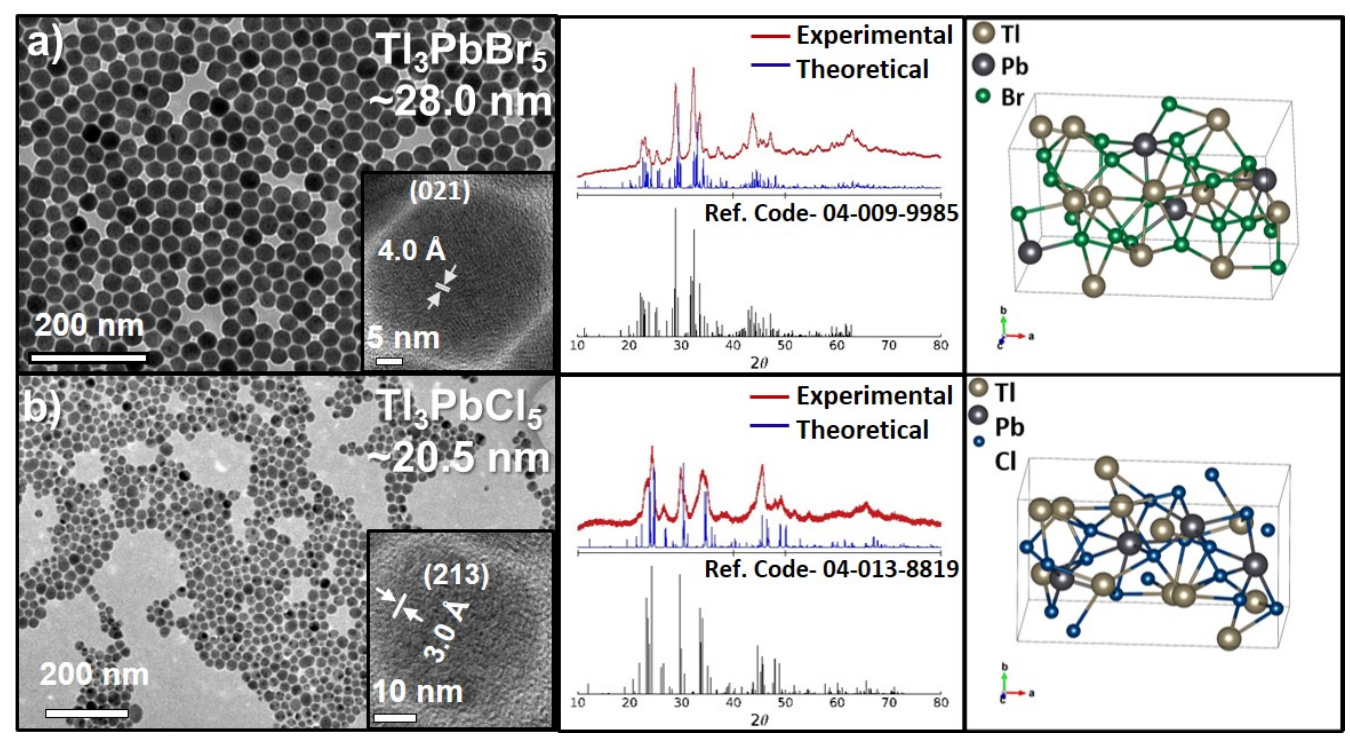

Figure 5.6: $\mathrm{HR}$-TEMs for $a) \approx 28.0 \mathrm{~nm}$ of $\left.\mathrm{Tl}_{3} \mathrm{PbBr}_{5}, b\right) \approx 20.5 \mathrm{~nm}$ of $\mathrm{Tl}_{3} \mathrm{PbCl}_{5} \mathrm{NCs}$, XRD spectra of these same NCs, matched to index spectra (X'Pert HighScore Plus), as well as spectra theoretically modelled in VESTA, and their respective periodic crystal structures, which have been optimised in VASP, for a) $\mathrm{Tl}_{3} \mathrm{PbBr}_{5}$, and b) $\mathrm{Tl}_{3} \mathrm{PbCl}_{5}$. Computational modeling was conducted by Dani Z. Metin. Copyright, American Chemical Society 2018.

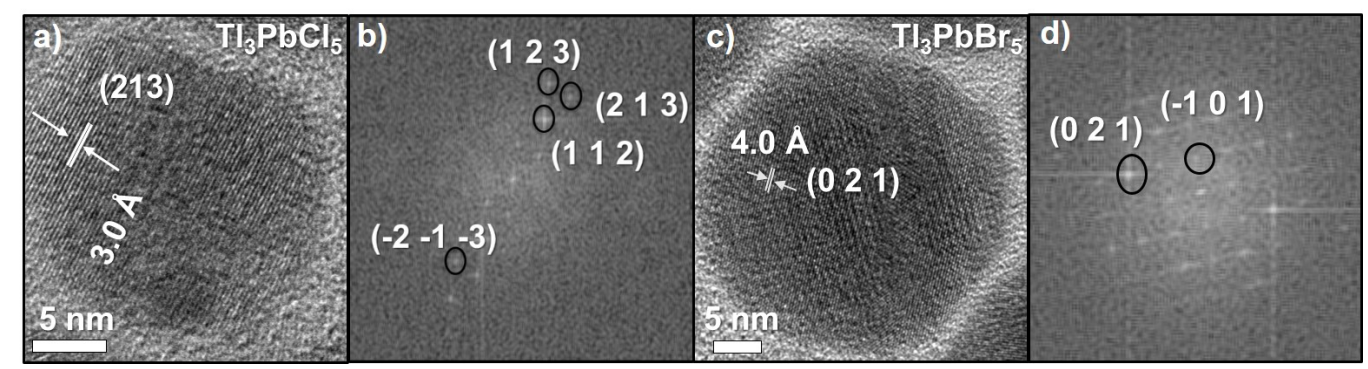

Figure 5.7: High resolution TEM micrographs of a) $\mathrm{Tl}_{3} \mathrm{PbCl}_{5}$, and c) $\mathrm{Tl}_{3} \mathrm{PbBr}_{5}$ NCs along with the FFT of crystal planes. CaRIne was used to make the crystal structure and the FFT was compared with the high rest TEM FFT $(b, d)$. NCs were found to be melting under electron beam during the imaging, which limits the STEM mapping of these particles. Copyright, American Chemical Society 2018. 
were fully optimised using first-principle calculations as described in Section 5.3.1.2. That crystal structure was then used to extract theoretical XRD spectra shown in Figure 5.5. The middle panel of Figure 5.6 shows the XRD spectra of $\mathrm{Tl}_{3} \mathrm{PbBr}_{5}$ and $\mathrm{Tl}_{3} \mathrm{PbCl}_{5}$ NCs measured by PANalytical X-ray diffractometer. The XRD spectra for $\mathrm{Tl}_{3} \mathrm{PbBr}_{5}$ and $\mathrm{Tl}_{3} \mathrm{PbCl}_{5}$, were matched to the index spectra using PANalytical X'Pert Highscore Plus software data file 04-009-9985 and 04013-8819. XRD spectra were also matched with the theoretically modeled spectra in VESTA. The XRD peaks were also found to be broadened compared to the bulk index peaks according to the Scherrer equation 1.39. Lattice fringes in NCs are clearly visible in high resolution TEM images (Figure 5.6). The visible lattice fringes of $\mathrm{Tl}_{3} \mathrm{PbBr}_{5}$ and $\mathrm{Tl}_{3} \mathrm{PbCl}_{5}$ were matched to the (021) and (213) crystal plane of the lattice structures with space group P212121 and P41212 (Figure 5.6). In order to proof check the crystal structure, CaRIne software was used to draw the crystal structure with given lattice parameters from XRD data to create the fast Fourier transform (FFT). Figure 5.7 shows the FFT from high resolution TEM image of $\mathrm{Tl}_{3} \mathrm{PbBr}_{5}$ and $\mathrm{Tl}_{3} \mathrm{PbCl}_{5}$ NCs. The FFT from the TEM image and the FFT from the crystal structure drawn by CaRIne were compared. The matching of lattice planes in fast Fourier transform (FFT) with standard crystal structure of $\mathrm{Tl}_{3} \mathrm{PbBr}_{5}$ and $\mathrm{Tl}_{3} \mathrm{PbCl}_{5}$ obtained by XRD data file also supports the XRD analysis (Figure 5.7). Similar to $\mathrm{Tl}_{3} \mathrm{PbI}_{5} \mathrm{NCs}$, all of these results confirm the formation of a orthorhombic crystal structure for $\mathrm{Tl}_{3} \mathrm{PbBr}_{5}$ and a tetragonal crystal structure for $\mathrm{Tl}_{3} \mathrm{PbCl}_{5} \mathrm{NCs}$.

Denysyuk et al. reported a high temperature tetragonal phase for $\mathrm{Tl}_{3} \mathrm{PbBr}_{5}$ [269]. This phase transition occurs at $245^{\circ} \mathrm{C}$ [269]. The high temperature tetragonal phase was found to exhibit a smaller bulk band gap of $2.26 \mathrm{eV}$, whereas low temperature phase exhibits the bulk band gap of $3.06 \mathrm{eV}$ based on our theoretical calculation [269]. Similarly, $\mathrm{Tl}_{3} \mathrm{PbCl}_{5}$ also exists in two different tetragonal crystal phases with transition temperature occurring at $170{ }^{\circ} \mathrm{C}[270]$. Theoretical calculations suggested the high temperature phase has a band gap of 3.39 $\mathrm{eV}$ [270]. However, a band gap of $2.73 \mathrm{eV}$ was estimated in the low temperature phase of $\mathrm{Tl}_{3} \mathrm{PbCl}_{5}$. It should be noted that we have only observed the low 


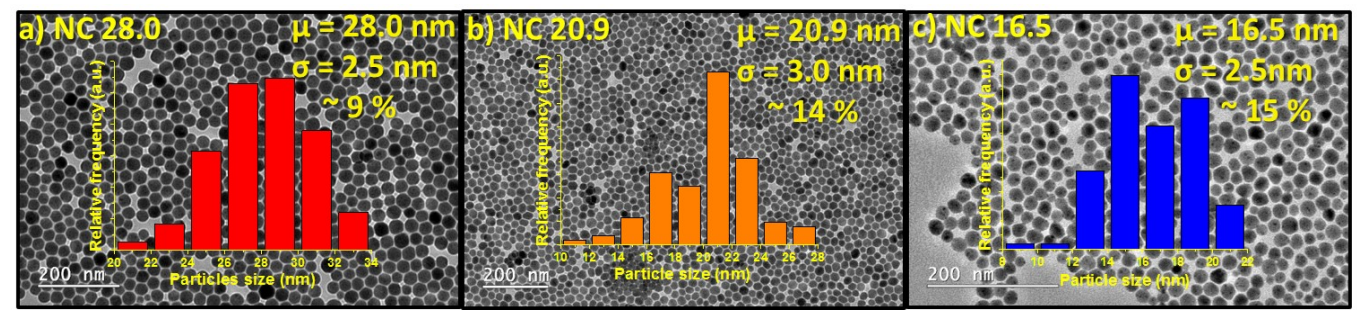

Figure 5.8: High resolution TEM micrographs of $\mathrm{Tl}_{3} \mathrm{PbBr}_{5} \mathrm{NCs}$ synthesised at three different hot-injection temperature (Table 5.1) to observe the size tuning. A histogram is created on top of each TEM image to calculate the particle size of a) $28.0 \pm 2.5, b) 20.9 \pm 3.0$, and c) $16.5 \pm 2.5 \mathrm{~nm}$. Particles were dissolved in hexane before the TEM sample preparation.

temperature phase of $\mathrm{Tl}_{3} \mathrm{PbCl}_{5}$ and $\mathrm{Tl}_{3} \mathrm{PbBr}_{5}$ NCs.

The particle size was measured by creating a histogram of each TEM image using ImageJ. NCs size for $\mathrm{Tl}_{3} \mathrm{PbBr}_{5}$ and $\mathrm{Tl}_{3} \mathrm{PbCl}_{5}$ was calculated to be $28.0 \pm 2.5$ and $20.5 \pm 2.7$ successively. The variation in the size of $\mathrm{Tl}_{3} \mathrm{PbBr}_{5}$ and $\mathrm{Tl}_{3} \mathrm{PbCl}_{5}$ is due to the hot-injection temperature. Size tuning is one of the major advantage of NCs because it allows us to tune the optical properties of that material up to some extent [271] To investigate the extent of size tuning, $\mathrm{Tl}_{3} \mathrm{PbBr}_{5} \mathrm{NCs}$ with three different sizes were synthesised. Size of these NCs was calculated to be $28.0 \pm 2.5,20.9 \pm 3.0$, and $16.5 \pm 2.5 \mathrm{~nm}$ (Figure 5.8). The monodispersity in these NCs was observed to be under $15 \%$, which is overlaid on top of each TEM image.

\subsubsection{Mixed halide $\mathrm{Tl}_{3} \mathrm{PbY}_{5-x} \mathrm{Br}_{x}(\mathrm{Y}=\mathrm{Cl}, \mathrm{I}) \mathrm{NCs}$}

So far, shape of NCs, composition and size tuneability have been observed in this material. However, halide mixing was yet to be performed. Similar to any perovskite, $\mathrm{Tl}_{3} \mathrm{PbX}_{5}$ also showed the advantage of halogen mixing. Mixed halide NCs were synthesised by mixing halide precursor, as per the instruction given in Table 5.1. Figure 5.9 shows the TEM micrographs of mixed halide NCs. NCs 


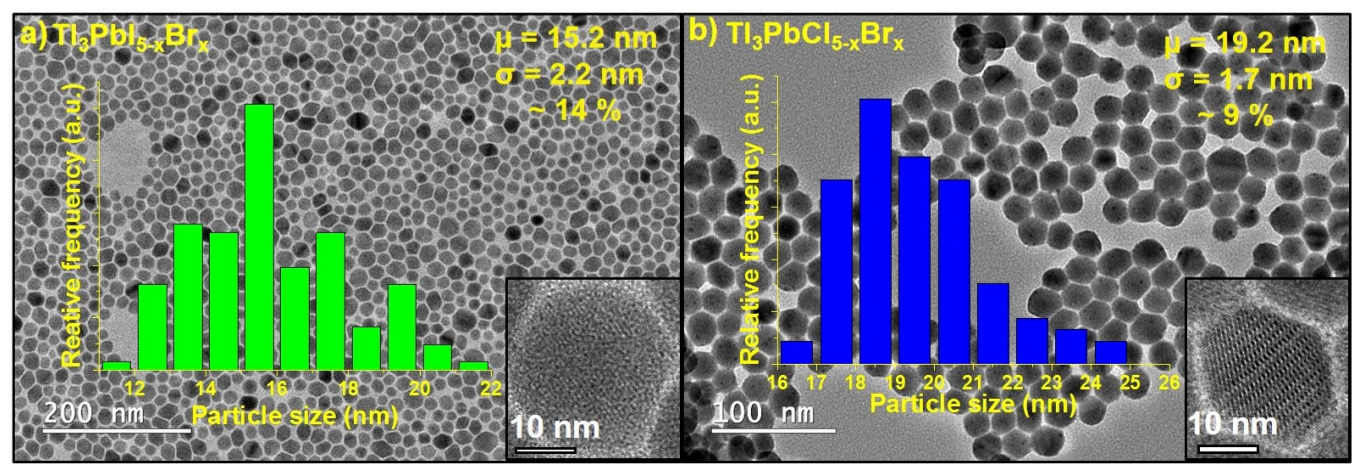

Figure 5.9: Histogram with the TEM micrograph of a) $\mathrm{Tl}_{3} \mathrm{~Pb}\left(\mathrm{I}_{0.43} / \mathrm{Br}_{0.57}\right)_{5} \mathrm{NCs}$ synthesised at $140^{\circ} \mathrm{C}$. NCs have $15.2 \mathrm{~nm}$ diameter with $2.2 \mathrm{~nm}$ standard deviation. b) $\mathrm{Tl}_{3} \mathrm{~Pb}\left(\mathrm{Cl}_{0.36} / \mathrm{Br}_{0.64}\right)_{5} \mathrm{NCs}$ synthesised at $130{ }^{\circ} \mathrm{C}$. NCs have $19.2 \mathrm{~nm}$ diameter with $1.7 \mathrm{~nm}$ standard deviation. ImageJ was used to create the histogram. Copyright, American Chemical Society 2018.

showed similar shape like pure halide NCs with particle size of $15.2 \pm 2.2 \mathrm{~nm}$ for $\mathrm{Tl}_{3} \mathrm{PbI}_{5-x} \mathrm{Br}_{x}$ and $19.2 \pm 1.7 \mathrm{~nm}$ for $\mathrm{Tl}_{3} \mathrm{PbCl}_{5-x} \mathrm{Br}_{x}$. Particle size was calculated by creating a histogram for each TEM image, which is plotted on top of the Figure 5.9. The ratio between halides was calculated by taking the average from TEM EDS data.

As can be seen in the Figure 5.10, the XRD peaks are broadened compared to the theoretical modelled spectra, due to the Scherrer equation 1.39. XRD spectra of mixed halides are first compared with theoretically simulated spectra of pure halide (Figure 5.10). Figure 5.10a shows the comparison between $\mathrm{Tl}_{3} \mathrm{PbI}_{5-x} \mathrm{Br}_{x}$ experimental XRD and theoretically modelled $\mathrm{Tl}_{3} \mathrm{PbI}_{5}$. The graph shows a good match between two highest intensity peak of experimental and theoretical spectra. However, From $2 \theta=20^{\circ}-23^{\circ}$ we observe an experimental peak that is not well described to experiment on the basis that two theoretical peaks of higher relative intensity lie outside of the range where the experimental peak is, and smaller theoretical peaks lie in between this. Figure $5.10 \mathrm{~b}$ shows the comparison of $\mathrm{Tl}_{3} \mathrm{PbI}_{5-x} \mathrm{Br}_{x}$ experimental XRD with theoretically modelled $\mathrm{Tl}_{3} \mathrm{PbBr}_{5}$. The graphs show a right shift in two highest intensity 
a)

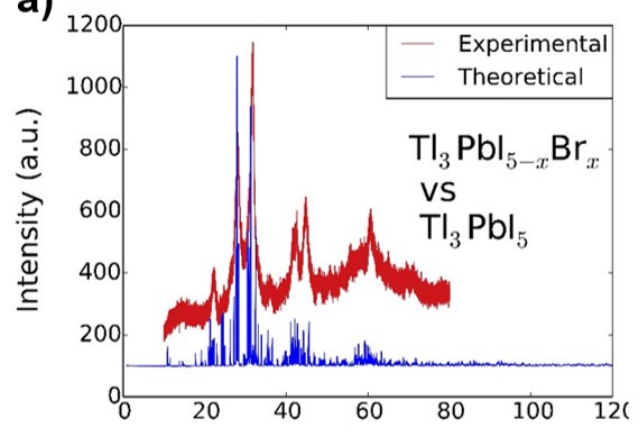

c)

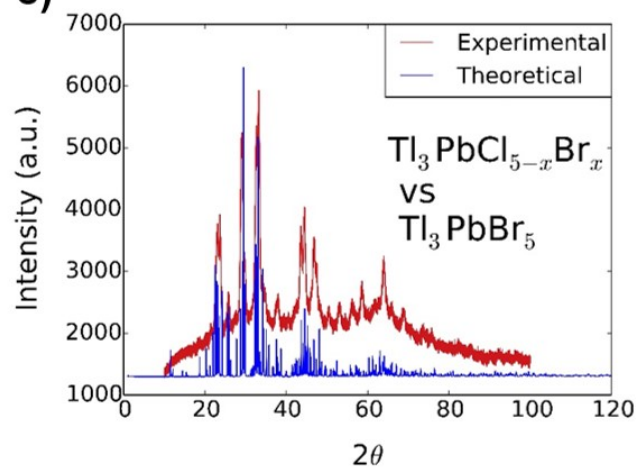

b)

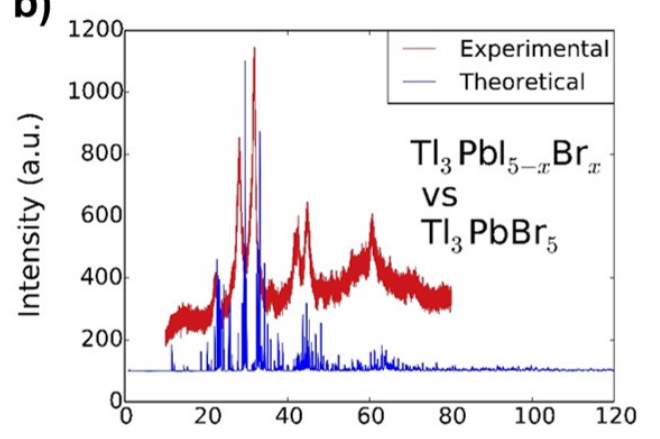

d)

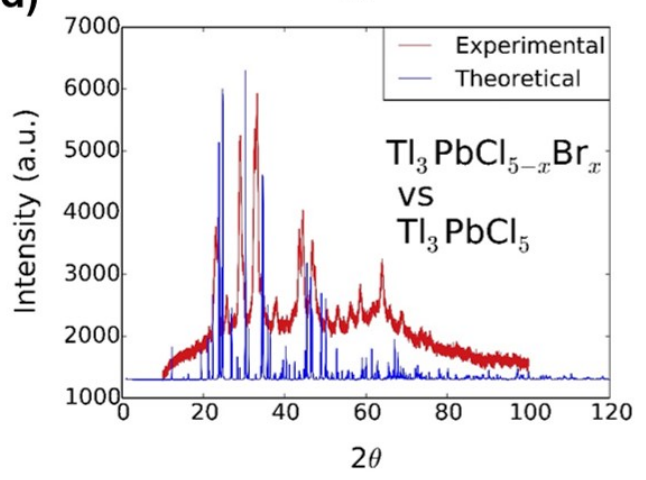

Figure 5.10: Comparison of experimental mixed halide XRD to theoretical pure halide XRD, whereby a) $\mathrm{Tl}_{3} \mathrm{PbI}_{5-x} \mathrm{Br}_{x}$ vs $\mathrm{Tl}_{3} \mathrm{PbI}_{5}$, b) $\mathrm{Tl}_{3} \mathrm{PbI}_{5-x} \mathrm{Br}_{x}$ vs $\mathrm{Tl}_{3} \mathrm{PbI}_{5}, c$ ) $\mathrm{Tl}_{3} \mathrm{PbCl}_{5-x} \mathrm{Br}_{x}$ vs $\mathrm{Tl}_{3} \mathrm{PbBr}_{5}$, and d) $\mathrm{Tl}_{3} \mathrm{PbCl}_{5-x} \mathrm{Br}_{x}$ vs $\mathrm{Tl}_{3} \mathrm{PbCl}_{5}$. Copyright, American Chemical Society, 2018.

peak of $\mathrm{Tl}_{3} \mathrm{PbI}_{5-x} \mathrm{Br}_{x}$ in comparison of $\mathrm{Tl}_{3} \mathrm{PbBr}_{5}$ standard spectrum. The next two theoretical peaks $\left(2 \theta=40^{\circ}-50^{\circ}\right)$ of lesser intensity were also not found to be matched but showed a shift towards higher $2 \theta$. Overall, We see a reduced quality fit with the experimental XRD of mixed halide in comparison of the pure halide standard spectra.

The lower panel of Figure 5.10 shows the XRD spectra of $\mathrm{Tl}_{3} \mathrm{PbCl}_{5-x} \mathrm{Br}_{x}$ compared with theoretically modelled spectra of $\mathrm{Tl}_{3} \mathrm{PbBr}_{5}$ and $\mathrm{Tl}_{3} \mathrm{PbCl}_{5}$. In Figure 5.10d, the mixed halide XRD spectra shows a poor fit with the theoretically modelled XRD of $\mathrm{Tl}_{3} \mathrm{PbCl}_{5}$, as all of the high intensity peaks are shifted towards higher angle with respect to the mixed halide spectra. However, The $\mathrm{Tl}_{3} \mathrm{PbBr}_{5}$ 


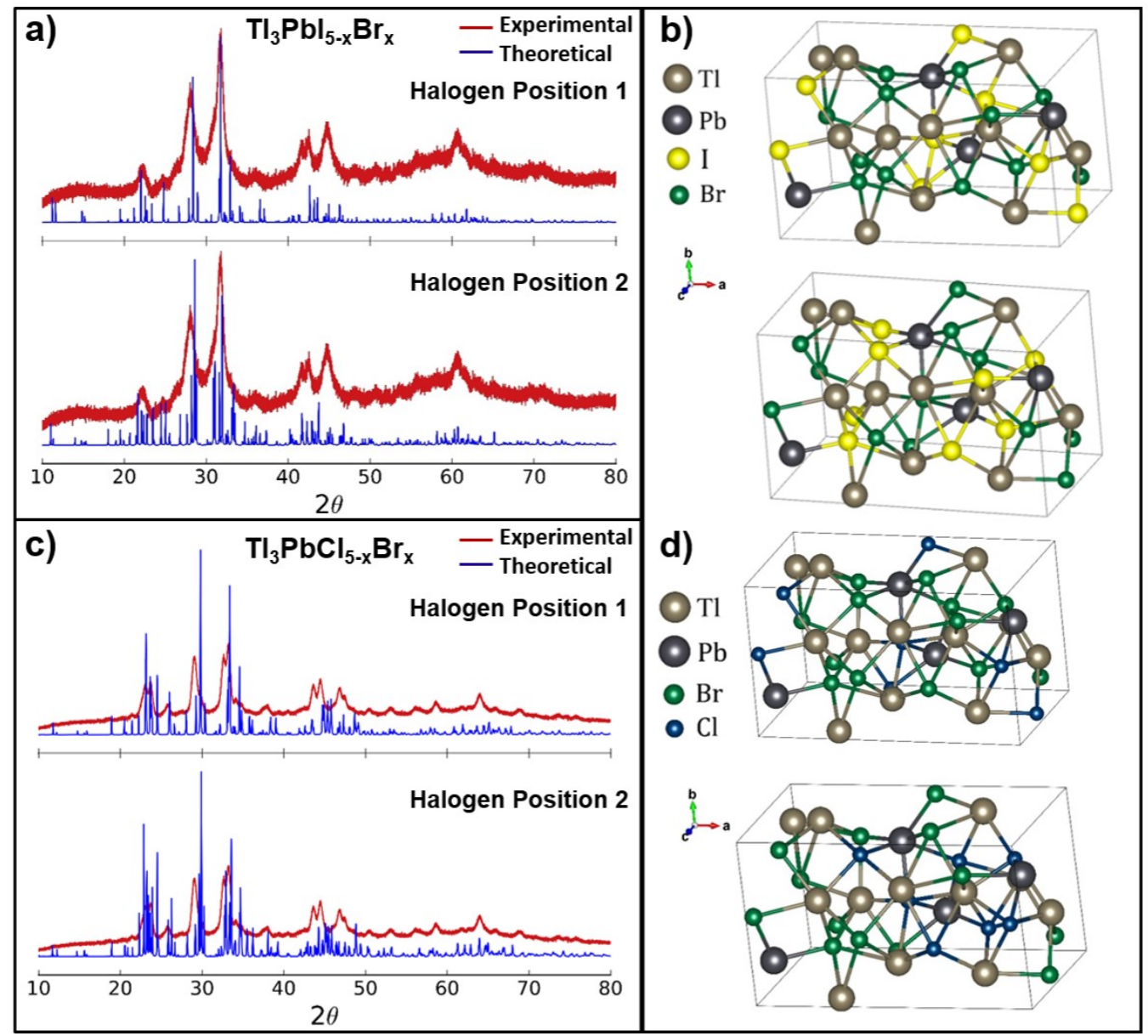

Figure 5.11: Comparison of experimental mixed halide XRD to the theoretical mixed halide XRD of a) $\mathrm{Tl}_{3} \mathrm{PbI}_{5-x} \mathrm{Br}_{x}$ and c) $\mathrm{Tl}_{3} \mathrm{PbCl}_{5-x} \mathrm{Br}_{x}$ NCs. Crystal structure for $b$ ) $\mathrm{Tl}_{3} \mathrm{PbI}_{5-x} \mathrm{Br}_{x}$ and d) $\mathrm{Tl}_{3} \mathrm{PbCl}_{5-x} \mathrm{Br}_{x}$ for two different halogen position. Courtesy of Dani Z. Metin for computational modeling.

spectrum shows a good agreement with the experimental $\mathrm{Tl}_{3} \mathrm{PbCl}_{5-x} \mathrm{Br}_{x}$ data. Based on the superior fit of the $\mathrm{Tl}_{3} \mathrm{PbBr}_{5}$ over $\mathrm{Tl}_{3} \mathrm{PbCl}_{5}$, we proposed that the NCs are ordered in an orthorhombic structure. Therefore, orthorhombic crystal structures of mixed halide were computationally constructed for the further comparison of XRD data.

To analyse the XRD spectra of mixed halide NC samples in details, we compared 
them with the theoretically simulated spectra of mixed halide, which were produced in VESTA using structures descended from the bulk low temperature orthorhombic crystal structure of $\mathrm{Tl}_{3} \mathrm{PbBr}_{5}$ [268]. The structure was optimised using the Vienna Ab-Initio Simulation Package (VASP) with two random variation in halide positions, named the Halogen Position 1 and 2 in the Figure 5.11 [272-274]. Lattice parameters of Halogen Position 1 are $a=15.77, b=9.10$, $\mathrm{c}=8.70 \AA$ for $\mathrm{Tl}_{3} \mathrm{PbI}_{5-x} \mathrm{Br}_{x}$ and $\mathrm{a}=15.0, \mathrm{~b}=8.70, \mathrm{c}=8.29 \AA$ for $\mathrm{Tl}_{3} \mathrm{PbCl}_{5-x} \mathrm{Br}_{x}$. Similarly, lattice parameters of Halogen Position 2 are $\mathrm{a}=15.63, \mathrm{~b}=9.35, \mathrm{c}=8.59 \AA$ for $\mathrm{Tl}_{3} \mathrm{PbI}_{5-x} \mathrm{Br}_{x}$ and $\mathrm{a}=14.88, \mathrm{~b}=8.81, \mathrm{c}=8.27 \AA$ for $\mathrm{Tl}_{3} \mathrm{PbCl}_{5-x} \mathrm{Br}_{x}$. Further details on theoretical modeling conducted by Dani Z. Metin under the guidance of Prof Nicola Gaston at University of Auckland, can be found in the supporting information of our published report [8]. Figure 5.11a shows the XRD spectrum of $\mathrm{Tl}_{3} \mathrm{PbI}_{5-x} \mathrm{Br}_{x}$, as one can see that there is a superior fit to the peaks of highest relative intensity over that of the pure halide case for both halogen substitution case. It is clear from the XRD that there is a good reproduction of the trends seen in the experimental XRD spectra. The shape of the XRD is well matched with the experimental data. However, the number of the peaks and their relative intensity are represented in the spectra to be affected by the halogen position. A similar trend was observed in the XRD spectrum of $\mathrm{Tl}_{3} \mathrm{PbCl}_{5-x} \mathrm{Br}_{x} \mathrm{NCs}$ (Figure 5.11c) and the spectrum fits reasonably well with the theoretically simulated XRD spectra. As can be seen in Figure 5.11c, there are only minor changes in the peak positioning of theoretical XRD with halogen substitution, possibly due to the periodic disorder in halogen positions. Thus, the XRD spectra of $\mathrm{Tl}_{3} \mathrm{PbI}_{5-x} \mathrm{Br}_{x}$ and $\mathrm{Tl}_{3} \mathrm{PbCl}_{5-x} \mathrm{Br}_{x}$ NCs were found to match with their corresponding theoretically simulated crystal structures. However, the distribution of $\mathrm{Br}$ and $\mathrm{I}$ in $\mathrm{Tl}_{3} \mathrm{PbI}_{5-x} \mathrm{Br}_{x}$ was not well ordered since high-order peaks could not be clearly observed [8]. 


\subsubsection{Optical Characterization}

Optical spectroscopy can give the details of material's properties, such as band gap, emission spectra, size distribution, and carrier life time. As the shape, size, and composition in the material changes, there should be a change in the band gap of the NCs. Each NC samples were diluted in hexane for the UV-Vis and PL measurements. A Cary $50 \mathrm{UV}$-Visible spectrophotometer was used to conduct the UV-Vis spectroscopy measurement. PL measurements were carried out using SP2500 from Princeton Instruments equipped with an intensified CCD (PIMAX 3 by Princeton Instruments) by Dr Kai Chen at Victoria University of Wellington.

\subsubsection{Absorption Spectroscopy}

Absorption spectra for compositionally tuned NCs and NWs are shown in Figure 5.12a. NWs showed the first absorption peak at relatively higher wavelength, $440 \mathrm{~nm}$. Similar to other semiconductor NCs, the pure $\mathrm{Tl}_{3} \mathrm{PbX}_{5} \mathrm{NCs}$ displayed evidence of a strong first direct transition peak, which is a typical peak of NCs under weak to intermediate confinement regime [20, 188]. First absorption peak for each absorption spectrum in Figure 5.12 was fitted with a Gaussian curve in Origin 9.1 and the width of the peak defines the size distribution in NCs. As this material has the indirect band gap, therefore, an indirect transition at higher wavelength was also observed in these NCs. The indirect transition was also observed in the bulk crystals of $\mathrm{Tl}_{3} \mathrm{PbCl}_{5}, \mathrm{Tl}_{3} \mathrm{PbBr}_{5}$, and $\mathrm{Tl}_{3} \mathrm{PbI}_{5}[251,258,270]$. It can be seen from the absorption spectra (Figure 5.12a) that the absorption edge moves towards lower wavelength as the composition changes from I to $\mathrm{Cl}$ in $\mathrm{Tl}_{3} \mathrm{PbX}_{5}$ NCs. The mixed halide NCs were found to have larger FWHM for their first absorption Gaussian fitted peak, possibly due to surface defects in mixed phase NCs. The first absorption peak in mixed halide materials was found to be intermediate between their respective pure halide NCs. Thus, the halide mixing and composition dependent band gap tuning 
allowed us to tune the optical properties from visible blue to the UV spectral region (Figure 5.12a).

Similar to other perovskite, halide composition tuning permitted the band gap tuning in these NCs [20]. Therefore, the change in size, which was performed in Section 5.3.1.3, should also display the band gap tuning up to some extent in $\mathrm{Tl}_{3} \mathrm{PbBr}_{5}$ NCs. Figure $5.12 \mathrm{~b}$ shows the absorption spectra of size tuneable $\mathrm{Tl}_{3} \mathrm{PbBr}_{5}$ NCs. The TEM images of same NCs are shown in Figure 5.8. As one can see in Figure 5.12b, increase in the particle size leads to move the first absorption edge, and therefore the peak of Gaussian fit, towards the higher wavelength. NC16.5 showed the first absorption edge at $321 \mathrm{~nm}$, however NC28.0 showed the red shifted first absorption edge at $333 \mathrm{~nm}$. Therefore, both composition and size dependent optical properties were observed in these NCs.

To investigate the evolution of confinement in $\mathrm{Tl}_{3} \mathrm{PbBr}_{5}$ NCs. The theoretical values of the direct band gap (first absorption edge) at different particle size were calculated from the following form of the Brus equation $[185,186]$

$$
\begin{gathered}
E=E_{g}+\frac{\hbar^{2} \pi^{2} \mu_{e x}^{*}}{2 r^{2}}-\frac{1.8 e^{2}}{4 \pi \epsilon_{r} \epsilon_{0} r} \\
\mu_{e x}^{*}=\frac{1}{m_{e}^{*}}+\frac{1}{m_{h}^{*}}
\end{gathered}
$$

Where, $\mathrm{r}$ is the radius of particle, $\mu_{\mathrm{ex}}^{*}$ is the exciton reduced mass, $\varepsilon_{\mathrm{r}}$ is the relative permittivity, and $E_{g}$ is the bulk band gap. The fitting parameters include the bulk band gap and the reduced mass of the exciton which were permitted to vary, while the relative permittivity $\left(\varepsilon_{r}\right.$, bulk $\approx 30$ ) was initially fixed. The bulk direct band gap of $\mathrm{Tl}_{3} \mathrm{PbBr}_{5}$ was calculated to be $3.65 \mathrm{eV}$, which was in the agreement of the reported values [258]. The exciton reduces mass was calculated to be $0.0242 \mathrm{~m}_{0}$. The binding energy was found to be $57 \mathrm{meV}$ and $5.2 \mathrm{~nm}$ of Bohr radius. These values were found to be similar to $\mathrm{CsPbX}_{3}$ perovskite NCs; except the fact that the first direct transition is not the band edge transition [17]. Figure 5.12c shows the plot of theoretically calculated band gap of $\mathrm{Tl}_{3} \mathrm{PbBr}_{5}$ with 


\section{a) $\mathrm{Tl}_{3} \mathrm{PbX}_{5}$ composition tuning}

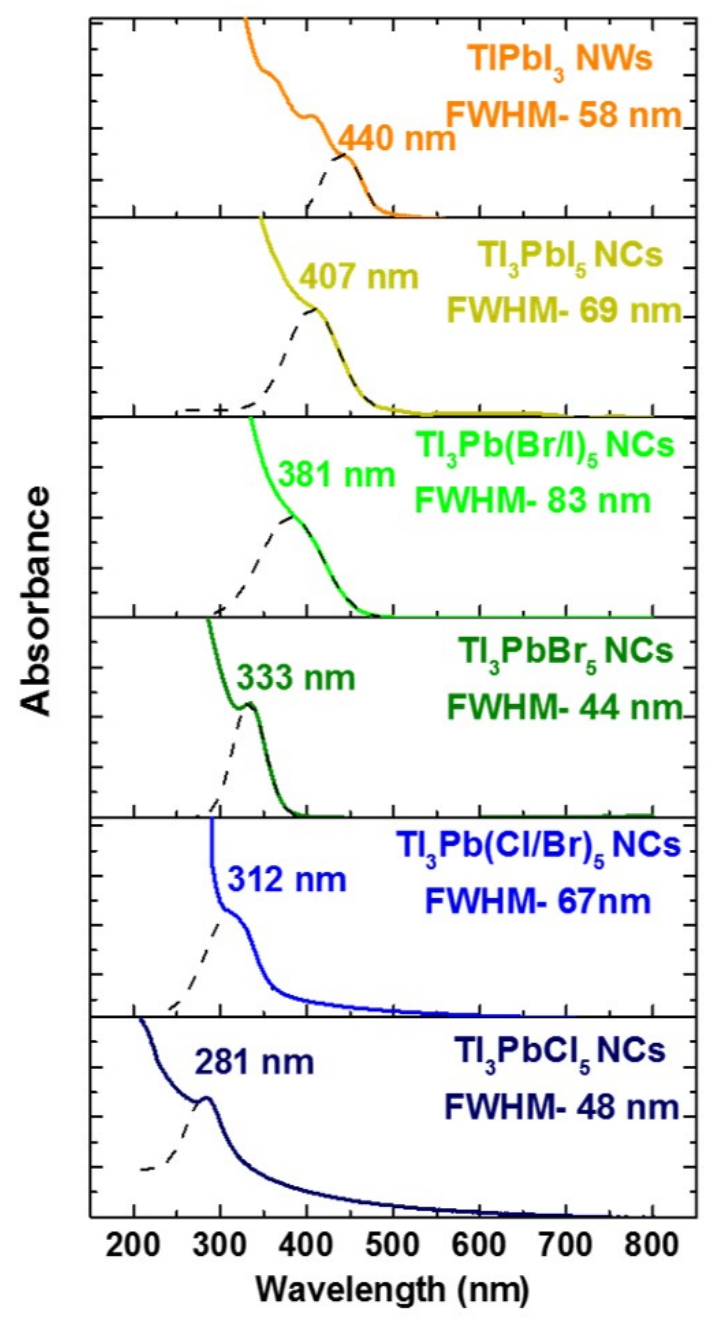

b) $\mathrm{Tl}_{3} \mathrm{~Pb} \mathrm{Br}$ size tuning

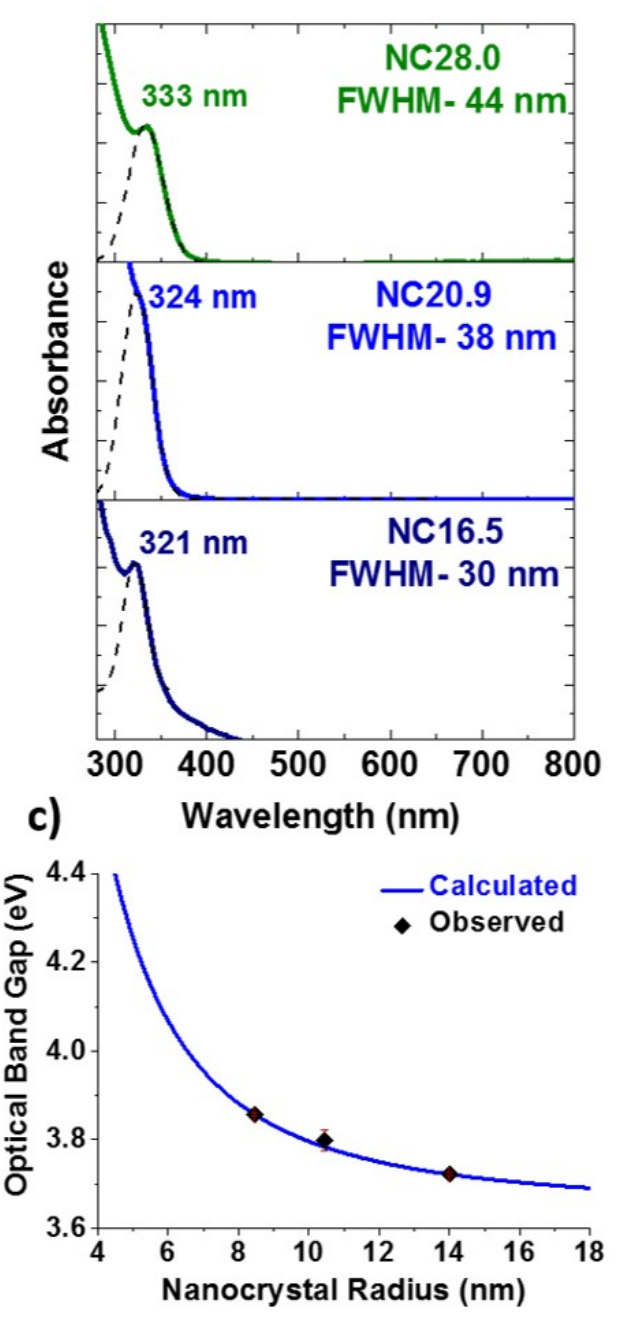

Figure 5.12: a) Absorption spectra of $\mathrm{TlPbI}_{3} \mathrm{NWs}$ and $\mathrm{Tl}_{3} \mathrm{PbI}_{5}, \mathrm{Tl}_{3} \mathrm{PbBr}_{5}$, and $\mathrm{Tl}_{3} \mathrm{PbCl}_{5} \mathrm{NCs}$ along with mixed halides $\mathrm{I} / \mathrm{Br}$ and $\mathrm{Cl} / \mathrm{Br}$ synthesised via a 1:1 reaction mixture, with a resulting $57 \%$ and $64 \%$ Br content confirmed by TEM EDS, respectively. b) Absorption spectra for size controlled $\mathrm{Tl}_{3} \mathrm{PbBr}_{5} \mathrm{NCs}$. First absorption peaks were identified using a Gaussian curve fit to determine the change in the direct band gap with size. (c) Plot of the direct band gap (as estimated from the first strong absorption peak) vs the NC radius, and fitted to the Brus Equation 5.2. Copyright, American Chemical Society 2018. 
a) Direct band gap

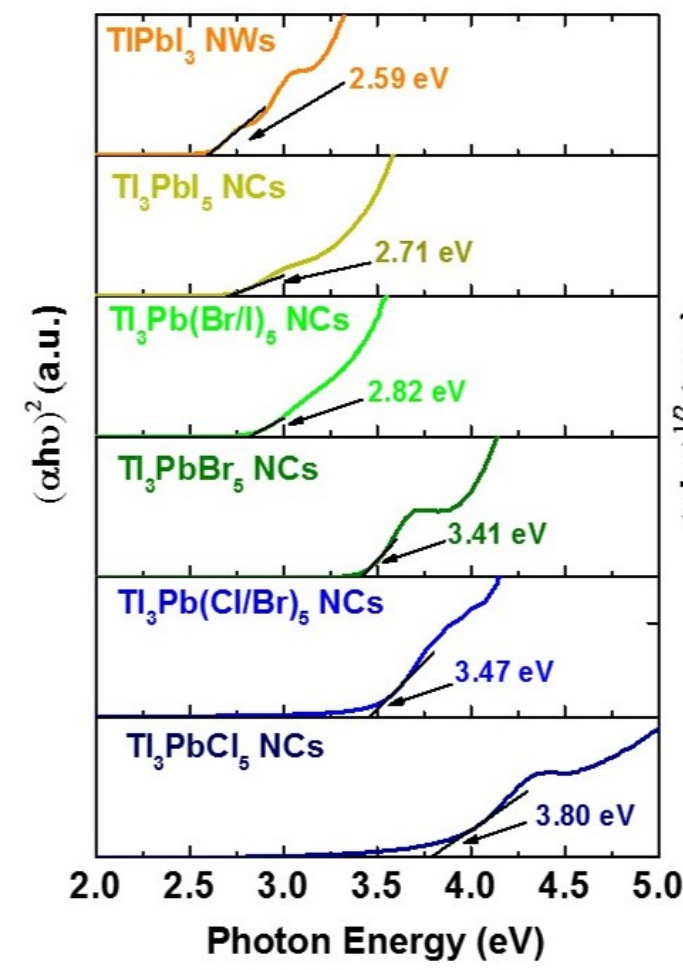

b) Indirect band gap

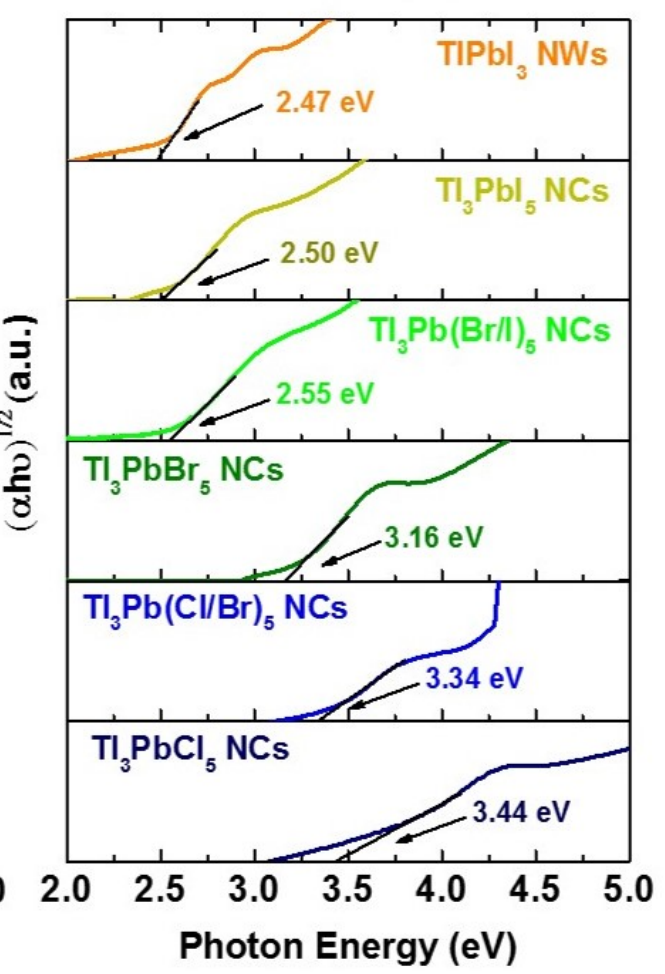

Figure 5.13: Tauc plot of $\mathrm{TlPbI}_{3} \mathrm{NWs}_{\text {and }} \mathrm{Tl}_{3} \mathrm{PbI}_{5}, \mathrm{Tl}_{3} \mathrm{PbBr}_{5}$, and $\mathrm{Tl}_{3} \mathrm{PbCl}_{5} \mathrm{NCs}$ along with mixed halides $\mathrm{I} / \mathrm{Br}$ and $\mathrm{Br} / \mathrm{Cl}$ shows the a) direct and b) indirect band edge transitions peaks as estimates of the band gap energy. Copyright, American Chemical Society 2018.

change in NC size. In addition, the experimentally observed band gap from the first absorption peak is also labelled on the same chart. If the radius of NCs is smaller than the Bohr radius then they should be in the strong confinement regime [188]. However, NCs larger than the Bohr radius should be in the weak confinement regime [188]. The radius of smallest $\mathrm{Tl}_{3} \mathrm{PbBr}_{5} \mathrm{NCs}$ was calculated to $\mathrm{be} \approx 8.2 \mathrm{~nm}$ (Figure 5.8) which is larger than the Bohr radius of $\mathrm{Tl}_{3} \mathrm{PbBr}_{5}(5.2$ $\mathrm{nm}$ ). Therefore, these NCs were found to be under weak confinement regime. Particle size smaller than $\approx 16.5 \mathrm{~nm}$ (radius- $8.2 \mathrm{~nm}$ ) could not be observed reliably and could be the part of future research. 
Table 5.2: A table of optical direct and indirect band gap in thallium lead halide NCs calculated by Tauc plot and compared with literature values of bulk of these materials [251, 258, 266, 270]

\begin{tabular}{|c|c|c|c|c|c|}
\hline $\begin{array}{c}\text { Material } \\
\text { name }\end{array}$ & $\begin{array}{c}\mathbf{1}^{\text {st }} \text { absorption } \\
\text { peak (eV) }\end{array}$ & $\begin{array}{c}\text { Tauc Fit } \\
\mathbf{E}_{g} \text { direct (eV) }\end{array}$ & $\begin{array}{c}\text { Tauc Fit } \\
\mathbf{E}_{g} \text { indirect (eV) }\end{array}$ & $\begin{array}{c}\text { Bulk } E_{g} \\
\text { indirect (eV) }\end{array}$ & $\begin{array}{c}\text { PL observed } \\
(\mathbf{e V})\end{array}$ \\
\hline $\mathrm{TlPbI}_{3} \mathrm{NWs}$ & 2.81 & 2.59 & 2.47 & 2.30 & - \\
$\mathrm{Tl}_{3} \mathrm{PbI}_{5}$ & 3.04 & 2.71 & 2.50 & 2.29 & $2.23,2.49$ \\
$\mathrm{Tl}_{3} \mathrm{~Pb}(\mathrm{Br} / \mathrm{I})_{5}$ & 3.25 & 2.82 & 2.55 & - & - \\
$\mathrm{Tl}_{3} \mathrm{PbBr}$ & 3.72 & 3.41 & 3.16 & 3.05 & - \\
$\mathrm{Tl}_{3} \mathrm{~Pb}(\mathrm{Br} / \mathrm{Cl})_{5}$ & 3.97 & 3.47 & 3.34 & - & - \\
$\mathrm{Tl}_{3} \mathrm{PbCl}_{5}$ & 4.41 & 3.80 & 3.42 & 3.39 & - \\
\hline
\end{tabular}

The optical band gaps in these material were calculated by constructing a Tauc plot (Figure 5.13) for direct and indirect band transition. The optical band gap values, observed in the Tauc plot, were in the agreement with the absorption edge position observed in Figure 5.12. $\mathrm{TlPbI}_{3}$ showed the highest direct and indirect band gap of 2.59 and $2.47 \mathrm{eV}$, respectively. The direct and indirect band gaps of $\mathrm{Tl}_{3} \mathrm{PbX}_{5}$ NCs were also found to be blue shifted as we moved up the halide series from I to $\mathrm{Cl}$. Band gaps of mixed halide NC were observed in between their parent pure halide NCs. All of these band gap values are compared with literature values of bulk of these materials and summarised in Table 5.2. Figure 5.14 shows the effect of composition on the direct band gap of the material. It can be seen in Figure 5.14, the overall band gap is turned from visible blue to UV regions (2.59-3.80 eV) by changing the halide composition in $\mathrm{Tl}_{3} \mathrm{PbX}_{5}$ NCs. Thus, $\mathrm{Tl}_{3} \mathrm{PbX}_{5}$ NCs showed the halide composition and size dependent band gap tuneability similar to other perovskite materials [20]. These tunable optical properties are beneficial for optoelectronic applications. 


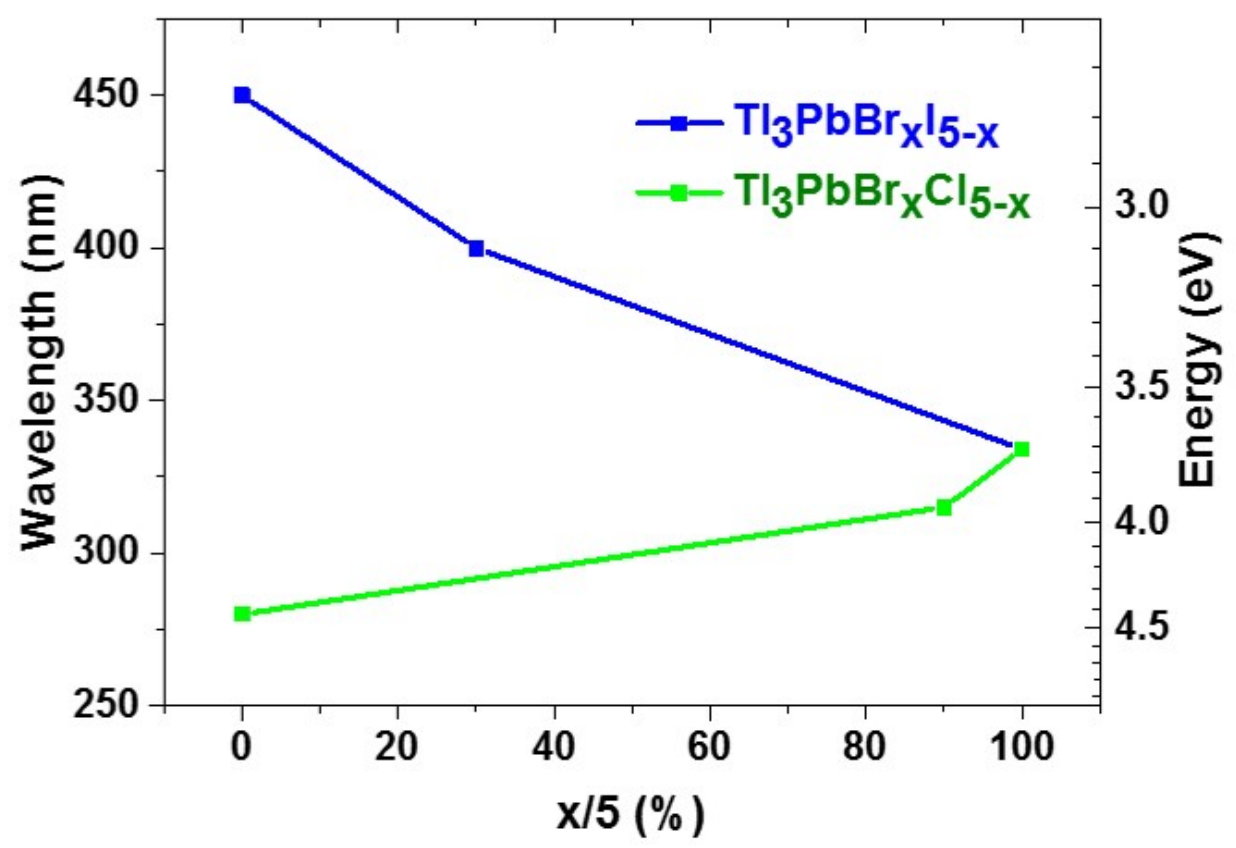

Figure 5.14: A chart showing the effect of composition on the direct band gap of the material, calculated by first absorption edge. Copyright, American Chemical Society 2018.

\subsubsection{Emission Spectroscopy}

All of these samples were characterised for emission properties. A PL spectroscopy was carried out by Dr Kai Chen under the guidance of Prof Justin Hodgkiss, at Victoria University of Wellington. No emission could be observed in any of the samples except $\mathrm{Tl}_{3} \mathrm{PbI}_{5} \mathrm{NCs}$. In this sample, a weak emission was measured at high pump excitation density of $\approx 42 \mu \mathrm{J} / \mathrm{cm}^{2}$. Figure 5.15 (left) shows the emission spectrum of $\mathrm{Tl}_{3} \mathrm{PbI}_{5}$ with the PL lifetime (right). The graph shows a broad band emission peak from 430 to $700 \mathrm{~nm}$. The sample was excite at $400 \mathrm{~nm}$ but due to the limitation of a $430 \mathrm{~nm}$ long pass filter, emission below $430 \mathrm{~nm}$ could not be observed. The emission spectrum did not appear to fit the profile of a trap emission in NCs. However, the curve is fitted into a combina- 


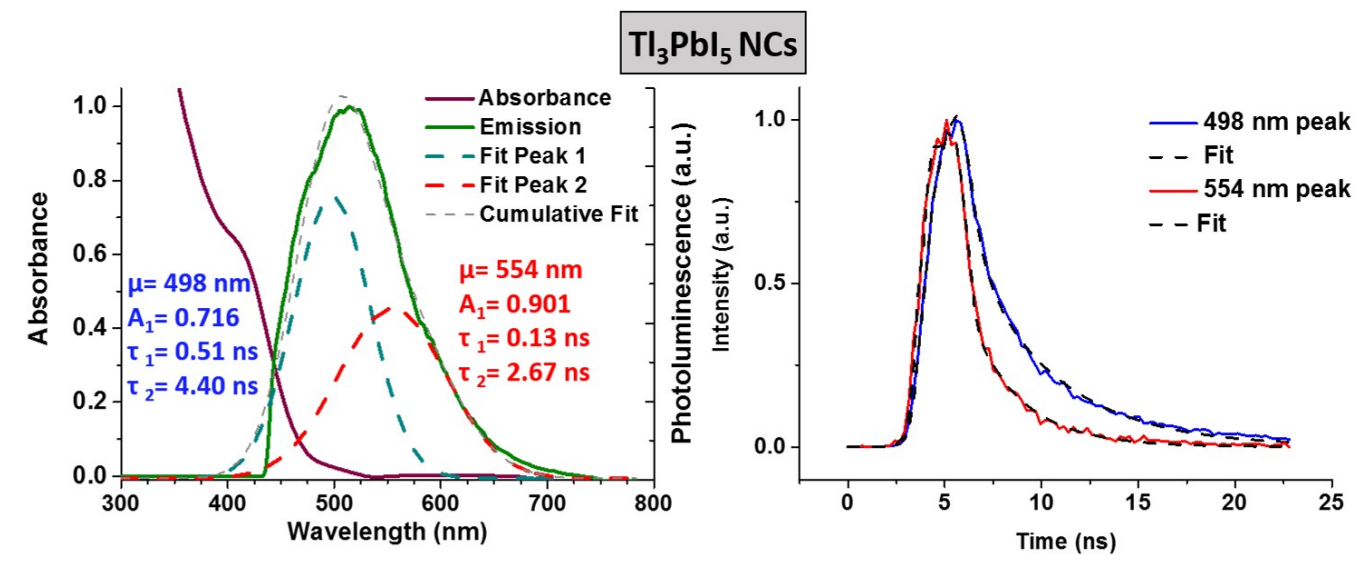

Figure 5.15: Emission spectrum of $\mathrm{Tl}_{3} \mathrm{PbI}_{5} \mathrm{NCs}$, observed at excitation density of $42 \mu \mathrm{J} / \mathrm{cm}^{2}$, shows the wide emission across the visible spectrum. The spectrum is fitted into two Gaussian curve when were then used to analyse the carrier lifetime of NCs. Copyright American Chemical Society, 2018.

tion of two Gaussian curves. Additionally, some small intensity was observed beyond $650 \mathrm{~nm}$, which is indicative of trap emission. This approach can be further justified by the presence of a visible shoulder to the curve, and the poor fit of a single Gaussian profile [275-277]. The broad band emission peak is fitted into two Gaussian peaks with emission at 554 and $498 \mathrm{~nm}$ Figure (5.15). The first fitted peak at $554 \mathrm{~nm}$ or $2.24 \mathrm{eV}$ accounts for $55 \%$ of the integrated emission signal. The emission at $2.24 \mathrm{eV}$ is in the agreement with the literature indirect band-gap energy of $2.29 \mathrm{eV}$ for emission at $300 \mathrm{~K}$ in bulk $\mathrm{Tl}_{3} \mathrm{PbI}_{5}$ [251]. The second Gaussian fitted peak at $498 \mathrm{~nm}$ or $2.48 \mathrm{eV}$ could be due to high energy trap sites for smaller NCs which have larger band gaps, or from the first direct transition observed in the UV-Vis analysis. (Figure 5.12). A direct-indirect energy contribution was previously observed in other perovskite analogues as well [277]. The PL kinetics at 554 and $498 \mathrm{~nm}$ are fitted with double exponential decay function in Figure 5.15b. In addition, they are numerically fitted by the convolution of the instrument response function with the double exponential decay by Dr Kai Chen. 


$$
l(\tau)=A_{1} \exp \left(-\frac{\tau}{\tau_{1}}\right)+\left(1-A_{1}\right) \exp \left(-\frac{\tau}{\tau_{2}}\right)
$$

Table 5.3: A table for the fitting values

\begin{tabular}{|c|c|c|}
\hline Values & $554 \mathrm{~nm}$ & $498 \mathrm{~nm}$ \\
\hline $\mathrm{A}_{1}$ & 0.906 & 0.7164 \\
$\tau_{1}$ & 0.1342 & 0.5143 \\
$\tau_{2}$ & 2.6747 & 4.3853 \\
\hline
\end{tabular}

In Table 5.3, $\mathrm{A}_{1}$ for Gaussian fitted 554 and $498 \mathrm{~nm}$ curve is 0.901 and 0.716 respectively. Therefore $\tau_{1}$ has higher contribution for decay path ways than $\tau_{2}$. These fitting values show the sub-nanosecond decay path way for $\tau_{1}$ and long decay path ways $\left(\tau_{2}\right)$ of 2.7 and $4.4 \mathrm{~ns}$ for the 554 and $498 \mathrm{~nm}$ PL peaks. As the larger percentage of the signal intensity was found to be decayed via $\tau_{1}$, which showed the shorter path ways, suggests that the material has a dominant, fast, and nonradiative decay path. This could be due to the interband trap or surface trap states, as the material showed very low quantum yield and there is no type-I shell coating to passivate the surface defects. Additionally, as an indirect band gap semiconductor, the presence of multiple trap sites should contribute to the nonradiative decay process. The difference in decay rates for these peaks suggest that two different processes are occurring. However, it is apparent that the emission mechanism from these materials is complex, and a detailed spectroscopic analysis may be needed to better assign each peak.

\subsection{Application}

As described in the Introduction 5.1, thallium lead halide have shown promising applications in semiconductor devices, such as solar cells, X-ray radiation detectors, photodetectors, piezoelectric sensors, lasers, and non linear optics 
when doped with rare earth materials [251-257]. Therefore, NCs of these materials should have even more promising potential applications. Here, we demonstrate the application of $\mathrm{TlPbI}_{3} \mathrm{NWs}$ as a wide band gap $(\approx 2.47 \mathrm{eV})$ photoconductor. The device fabrication process is explained in Section 5.2.5. The NWs solution $(20 \mathrm{mg} / \mathrm{mL})$ was spin-coated onto the substrate without any ligand exchange. After the spin coating of the layer, the film was washed with ethanol. Two terminal measurement was carried out on the device. The current was not measurable in dark. However, current in the order of nanoamps was observed in the room light (Figure 5.16a).

Figure $5.16 \mathrm{~b}$ shows the effect of broad band light intensity on the current through the device. As one can see in the graph, while the intensity of light increases, there is a sudden change in the current. This suggests the photo response in $\mathrm{TlPbI}_{3} \mathrm{NWs}$. In order to do the quantitative analysis, the sample was excited with a $405 \mathrm{~nm}$ laser diode $\left(6.4 \mathrm{~mW} / \mathrm{cm}^{2}\right)$, above the band gap of the semiconductor. Figure 5.16c shows the two-terminal conductivity of the film when illuminated with a $405 \mathrm{~nm}$ laser diode. The IV behaviour was observed to be ohmic, which can be attributed to the fact that the majority of the conduction between terminals occurs within the NWs themselves, with some minimal number of hopping events between NWs required to complete the circuit. At a field dependence beyond $5.0 \mathrm{~V}$, the graph displayed a completely linear behaviour with the electric field. In Figure 5.16, the conductivity changes from $5.0 \times 10^{-6} \mathrm{Sm}^{-1}$ at $300 \mathrm{~K}$ to $4.2 \times 10^{-7} \mathrm{Sm}^{-1}$ at $150 \mathrm{~K}$, reaching its minimum value and then further increases to $5.0 \times 10^{-7} \mathrm{Sm}^{-1}$ at $100 \mathrm{~K}$. Due to the limitation of equipment, the measurement was only conducted till $100 \mathrm{~K}$. The conduction pathway is most likely limited by the hopping, not the conduction through the NWs. Additionally, these are oleic acid/oleylamine capped NWs so the hops are going to be relatively longer range, which reinforces the point that the conduction is hop limited. It is noted that current could only be recorded in light illumination. Therefore, thermal carrier plays only a small role in the conduction. As the device is equally illuminated at all temperatures, the carrier density can be assumed constant. The decrease in the conductivity from temperature range 300 

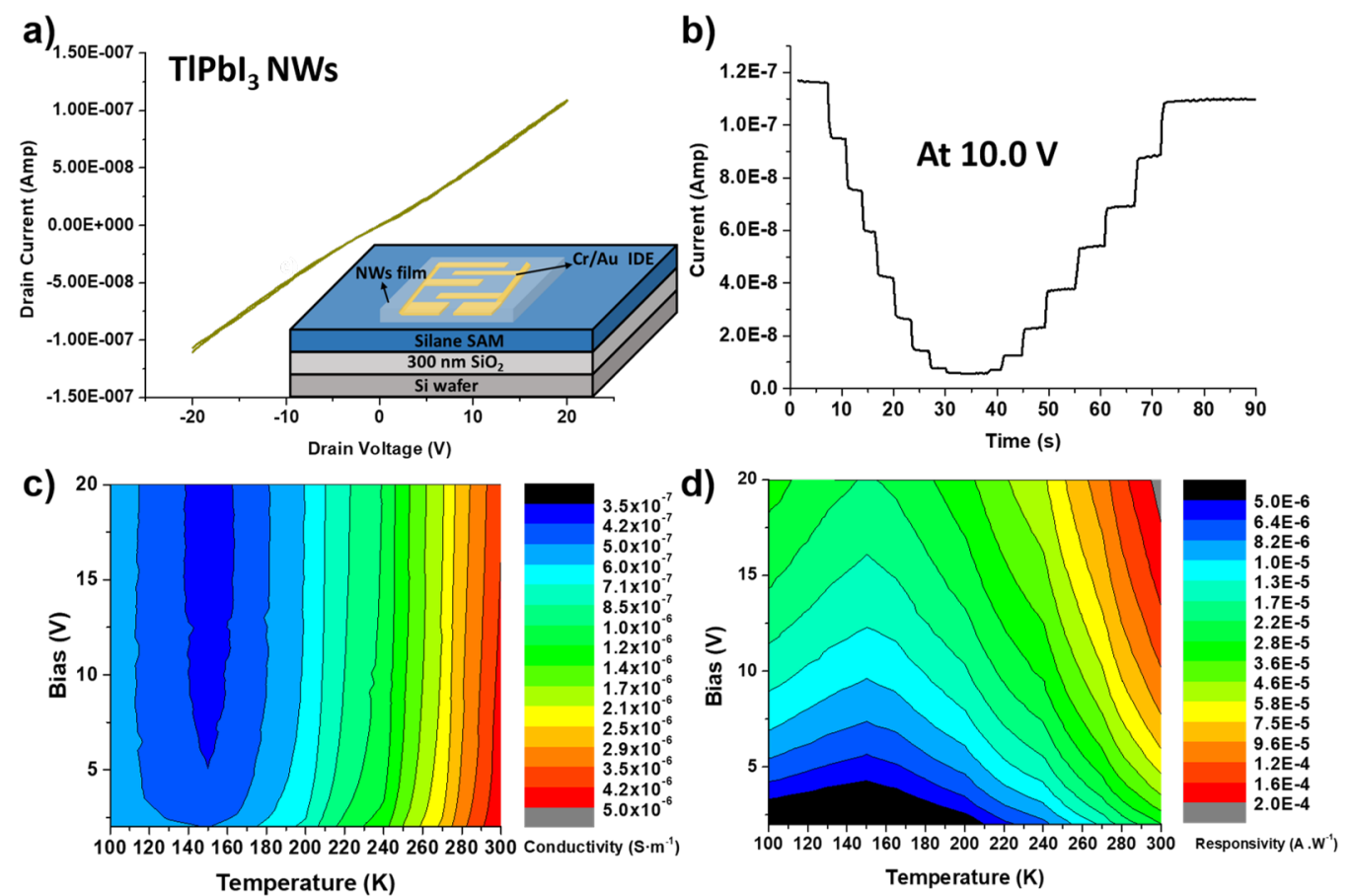

Figure 5.16: Electrical measurements on $\mathrm{TlPbI}_{3} \mathrm{NWs}$ a) Device structure and IV curve under constant light intensity, b) a curve showing the change in current with changing the broad band light intensity at $10.0 \mathrm{~V}, \mathrm{c}$ ) a contour map of the conductivity ( $S / m)$ vs temperature $(K)$ and voltage $(V)$, and d) a contour map showing the responsivity $(A / W)$ vs temperature $(K)$ and voltage $(V)$. Device fabrication process is explained in Section 5.2.5. Copyright American Chemical Society, 2018.

$\mathrm{K}$ to $150 \mathrm{~K}$ is therefore most likely a result of decrease in hopping rate with temperature $(\exp (-1 / \mathrm{T})$ relationship) [278-280]. Below $150 \mathrm{~K}$, with an assumption of constant carrier density, the conductivity is most likely under a new hopping regime with a different $\mathrm{T}$ dependence $\left(\exp \left(-1 / \mathrm{T}^{1 / 4}\right.\right.$ relation), which means that the conductivity decreases very slowly with the decrease in temperature. However, carrier mobility should be increased in NWs with decrease in hopping rate, which results in an overall increase in the conductivity of NWs [278-280]. The conductivity mechanism in these NWs need an extensive future research. All of this behaviour shows that the NWs are intrinsic semiconductor and be- 
have as such when illuminated with the light above its band gap. Figure 5.16d shows the photoconductive response of the device with respect to the voltage and temperature. The responsivity shows a very similar behaviour to the conductivity. Low values of conductivity and responsivity were due to the insulating oleyl ligands. However, The photo response with change in light intensity (Figure 5.16b) of these insulating films suggested that these NWs are worthy of future investigation.

\subsection{Conclusion}

We have synthesised halide composition dependent $\mathrm{Tl}_{3} \mathrm{PbX}_{5} \mathrm{NCs}(\mathrm{X}=\mathrm{Cl}, \mathrm{Br}, \mathrm{I})$ NCs with varying the particle size by colloidal route. The size tuneability was achieved by varying the reaction temperature. Evolution of quantum confinement was also understood in size tunable $\mathrm{Tl}_{3} \mathrm{PbBr}_{5} \mathrm{NCs}$, where a weak confinement was observed in NC16.5. To a lesser degree, a band gap tuning was demonstrated with varying the particle size in these NCs. Halogen mixing was achieved during the synthesis to produce mixed halide NCs. A systematic XRD study with computation analysis was conducted to confirm the orthorhombic crystal structure for $\mathrm{Tl}_{3} \mathrm{PbI}_{5}$ and $\mathrm{Tl}_{3} \mathrm{PbBr}_{5} \mathrm{NCs}$ and a tetragonal crystal structure for $\mathrm{Tl}_{3} \mathrm{PbCl}_{5}$ NCs. These materials exhibit size and composition tuneable band gap (3.80-2.59 eV) with low estimated binding energy, which makes them a potential candidate for photodetector and wide band gap solar cell. The rare earth doping in $\mathrm{Tl}_{3} \mathrm{PbBr}_{5}$, such as $\mathrm{Er}^{+3}$ or $\mathrm{Pr}^{+3}$, could make them a feasible candidate for mid-infrared nonlinear optics, which is the part of future research $[252,256]$.

On the other side, we have also synthesised thallium lead iodide NWs with $\mathrm{ABX}_{3}$ perovskite structure. The NWs were achieved by varying the reaction temperature and the ligand concentration. Bulk of $\mathrm{TlPbI}_{3}$ is one of the promising candidates for X-ray radiation detector [255]. Therefore, the NWs of these mate- 
rials should be worthy of future investigation in the X-ray detectors. We have demonstrated an application of these NWs in wide band gap photoconductor. These materials showed superior stability over caesium or methylammonium based perovskite analogues. Although, these materials showed a very weak emission, but after the surface passivation or doping, they should have potential application in optoelectronic devices. This is the first use of thallium as a monovalent cation in a perovskite nanostructure. Group A replacements within the $\mathrm{ABX}_{3}$ structure are rare among the metal halide perovskites, and have thus far been limited to methylammonium, formamidinium, and caesium, with few exceptions. Here we suggest the possibility of a new family of thallium-based perovskite nanostructures with interesting and novel properties. 


\section{Chapter 6}

\section{Conclusion and Future Work}

Metal halide perovskite materials are potential alternative for silicon technology in solar cell applications and cadmium based QDs in emission based applications such as LEDs. Several types of solution processed nanostructured are being used in current research to improve the efficiency of optoelectronic devices. However, some of the issues in these materials were not clearly resolved. Colloidal NCs can be easily used in high-scale optoelectronic devices by taking the advantages of spray coating, ink-jet printing, and other solution processing techniques, which offer very cheap and larger scale production. Particularly, pure inorganic metal halide perovskites have not only shown high stability but also a relatively higher thermal stability than hybrid organic-inorganic perovskite materials.

$\mathrm{CsPbX}_{3}(\mathrm{X}=\mathrm{Cl}, \mathrm{Br}, \mathrm{I}) \mathrm{NCs}$ have shown very high quantum yield of up to $90 \%$, with tuneable optical properties, suggesting their application in optoelectronic devices. We synthesised size controlled $\mathrm{CsPbBr}_{3} \mathrm{NCs}$ with three different sizes of $\approx 8.6,7.3$, and $4.1 \mathrm{~nm}$. We understood the evolution of quantum confinement in these NCs using static state absorption and ultrafast transient absorption spectroscopy. The smallest NCs were found to be in strong confinement regime, due to the higher energy absorption edges, higher Stokes shift, and blue 
shift in emission peak. In addition, the particle diameter was smaller than the Bohr diameter ( $7 \mathrm{~nm}$ ) of $\mathrm{CsPbBr}_{3}$, which supported our observations on strong confinement in NC-4.1. However, the NC-7.3 sample was found to be in intermediate confinement regime and NC-8.6 was under weak confinement regime due to their diameter larger than Bohr-diameter. In addition, the transient absorption spectroscopy measurements support the strong confinement effect in smallest NCs as the multimodal GSB was observed in NC-4.1. These sizeconfined NCs showed emission peaks from 495 to $522 \mathrm{~nm}$. Therefore, we took the advantage of halide composition tuning and synthesised $\mathrm{CsPb}(\mathrm{Br} / \mathrm{Y})_{3}(\mathrm{Y}=$ $\mathrm{Cl}, \mathrm{I}) \mathrm{NCs}$. These composition dependent NCs displayed the emission through the entire visible spectrum (490-680 nm). These NCs showed high quantum yield and were implemented in the fabrication of LEDs. NWs of $\mathrm{CsPbX}_{3}$ were also synthesised but due to their poor performance and low quantum yield they were not further investigated for LEDs application. However, optimising the synthesis of these NWs with better purification processes could be the part of future research.

We constructed peNC-LEDs with EL through the entire visible region. For the first time, we have shown precise colour tuning in the red spectral region using $\mathrm{CsPb}(\mathrm{Br} / \mathrm{I})_{3} \mathrm{NCs}$. We systematically observed the red shift in the EL of these devices. Temperature dependent PL studies were carried out to see the change in the emission properties with temperature, which appeared to have no significant effect. Then, we conducted the voltage and time dependent study on these LEDs, and investigated that the change in EL is primarily the function of electric field. In addition, the rate of ion migration was also found to increase with increase in bromide content in $(\mathrm{CsPbBr} / \mathrm{I})_{3}$ and device with highest amount of bromide content displayed the EL peak splitting in green (516 nm) and red $(665 \mathrm{~nm})$ spectral region. Ionic separation, to form separately emitting $\mathrm{NC}$ domains in NC films (as opposed to thin films), has not previously been observed and indicates that there are further dynamics in NCs systems that may be worth exploring. With improvements in surface coating and chemistry, these results suggest that peNC-LEDs could achieve device metrics that 
are more competitive with those of commercial NC-LED technologies. Device function was found to be highly dependent on voltage. However, the real dynamics within the device may be significantly more complex. Band bending and charge injection will affect the internal electric field at the interface, as will the differences and changes in the local dielectric in the NCs, the ligands and the surrounding organic semiconductors. We were only able to probe at macro level and a more realistic model may be warranted to fully understand the ion separation phenomenon within these devices.

Even after displaying high PL quantum yield, LEDs based on perovskite NCs showed very low external quantum efficiency $(<0.21 \%)$. This is possibly due to bulky ligands, larger exciton binding energies, and surface defects. Therefore, we investigated a pure inorganic quasi-2D (Ruddlesden-Popper phase) perovskite, which combines the advantage of colour purity and tuneability of nanomaterials with the conduction properties of 3D crystals [20, 35-37]. For the first time, we have shown the colour tuning through the entire visible spectrum by halide mixing in $\mathrm{CsPbX}_{3} \mathrm{RP}$ perovskite materials. The colour tuning was also achieved by varying the number of monolayers (nanosheet thickness) in these materials. LEDs were also fabricated with significant improvements in the EQE and stability of these device compared to the peNC-LEDs. Specifically, these LEDs outperformed the literature values in the blue and blue-green spectral region. The maximum EQE was recorded to be $10.1 \%$ at $506 \mathrm{~nm}$. Even with these improvements, these mixed halide LEDs still showed colour instability to a relatively lesser degree due to ion migration. The ion migration can be suppressed either by creating a type-I core-shell structure or a surface passivation with suitable ligands, which could be the part of future research in Halpert group.

Nanostructured perovskite were proven to be a promising material, therefore researchers have drawn attention towards investigating novel perovskite by replacing A or B groups in the structure with other feasible atoms [22-25]. We also focused our research towards this direction to invent novel perovskite NCs 
for similar properties and applications. For the first time, We synthesised NCs of thallium lead halide, which is a wide band gap semiconductor. We demonstrated the shape-, size-, and composition-tuning in these NCs. We synthesised $\mathrm{TlPbI}_{3}$ perovskite NWs with an indirect band gap of $2.47 \mathrm{eV}$. Thallium lead halide NWs are potential candidates for highly efficient X-ray radiation detectors, piezoelectric sensors, and solar cells [254, 255, 257, 266]. We demonstrated the use of $\mathrm{TlPbI}_{3}$ as a photoconductor, where an increase in current was observed with increase in light intensity. However, $\mathrm{NWs}$ of $\mathrm{TlPbBr} \mathrm{Pb}_{3}$ and $\mathrm{TlPbCl}_{3}$ could not be synthesised at atmospheric pressure and could be the part of future research [263]. By varying the reaction conditions, we were able to synthesis the $\mathrm{NCs}$ of $\mathrm{Tl}_{3} \mathrm{PbX}_{5}$. The band gap of these materials were tuned from visible blue to UV region (2.59-3.80 eV) by varying the halide composition as well as size of NCs. A weak confinement was observed in these NCs, which was estimated using Brus equation. In addition, $\mathrm{Tl}_{3} \mathrm{PbI}_{5} \mathrm{NCs}$ displayed a weak broad band emission from 430 to $700 \mathrm{~nm}$, which was fitted in two different Gaussian peaks. However, a detailed spectroscopy analysis is still needed to better assign each peak. These NCs have potential applications in non linear optics when doped with suitable rare earth materials [251-253, 256]. The rare earth material doping, such as $\mathrm{Er}^{3+}$ or $\mathrm{Pr}^{3+}$, in these NCs is also the part of future research. In addition, building an X-ray detector using $\mathrm{TlPbI}_{3} \mathrm{NWs}$ is under the future research for the development of these materials in the Halpert Group.

Overall, we have fabricated the mixed halide composition dependent perovskite NC LEDs with EL mainly focused in the red spectral region by using $\mathrm{CsPb}(\mathrm{Br} / \mathrm{I})_{3}$ NCs. The ion migration mechanism in these NC LEDs was understood for the first time in this research. Furthermore, we constructed inorganic RP based perovskite LEDs with tuneabble EL through the entire visible spectrum. These inorganic RP perovskite based LEDs outperformed the literature value in the blue spectral region (460-490 $\mathrm{nm})$. Various possible perovskite nanocrystals were investigated and we successfully demonstrated the colloidal route synthesis of $\mathrm{TlPbI}_{3}$ perovskite $\mathrm{NWs}$ and $\mathrm{Tl}_{3} \mathrm{PbX}_{5} \mathrm{NCs}$ for the first time. The control over the NCs size shape and composition was displayed by varying the reaction 
conditions. Eventually, the application of $\mathrm{TlPbI}_{3} \mathrm{NCs}$ was demonstrated as a photoconductor.

$\mathrm{CsPbX}_{3}$ synthesised in Chapter 2 were not fully optimized as they showed poor emission, solubility, and agglomerated morphology. The optimization of these NWs for optoelectronic applications could be the part of a future research. For LEDs application, overcoming the ion migration effect completely is yet to be achieved and could be the part of future research. The conduction mechanism in $\mathrm{TlPbI}_{3} \mathrm{NWs}$ is not fully understood and a realistic modal is warrant to study the change in conductivity with temperature in $\mathrm{TlPbI}_{3} \mathrm{NWs}$. One of the other major application of $\mathrm{TlPbI}_{3}$ is in the radiation detector [255], which is yet to be explored and could be the part of future research in the Halpert group. In addition to that, rare earth materials doping in $\mathrm{Tl}_{3} \mathrm{PbX}_{5} \mathrm{NCs}$ for the application of non linear optics could be investigated in future. 


\section{Chapter 7}

\section{Appendix}

\subsection{Appendix 1- Improving the Performance of Per- ovskite Solar Cells using CIS NCs}

Parts of this research have been published in the following article:

Ma Y, Vashishtha P, Shivarudraiah SB, Chen K, Liu Y, Hodgkiss JM, Halpert JE. A Hybrid Perouskite Solar Cell Modified With Copper Indium Sulfide Nanocrystals to Enhance Hole Transport and Moisture Stability. Solar RRL, 2017

\subsubsection{Introduction}

The power conversion efficiency (PCE) of perovskite solar cells has been improved from $2.6 \%$ to $22 \%$ in less than a decade [15, 146, 153]. However, the stability of these solar cells against the moisture is still one of the biggest concerns. Several strategies were used to enhance the device stability, including mixed cations and encapsulation of perovskite layers with organic layer [281284]. Commonly used hole transport layers, such as spiro-MeOTAD and PTAA, 
were not found to be very efficient due to the doping of a hygroscopic element (Li) [285]. Dong et al. encapsulated perovskite layer with a thin layer of $\mathrm{Al}_{2} \mathrm{O}_{3}$, which resulted in the poor device performance due to the decomposition of perovskite during atomic layer deposition (ALD) process [286]. Another possible candidate to encapsulate perovskite layer is copper indium sulfide $\left(\mathrm{CuInS}_{2}\right)$ NCs. CuInS 2 can be deposited by solution processing technique and are very robust against moisture [287-289]. Additionally, $\mathrm{CuInS}_{2}$ NCs can be synthesised as a p-type semiconductor for better hole transport [287]. In this project, we synthesised $\mathrm{CuInS}_{2}$ NCs by colloidal route adopted from literature [290]. These NCs were then used between perovskite and spiro-MeOTAD (HTL) layer. The incorporation of $\mathrm{CuInS}_{2}$ layer not only enhanced the moisture stability but also improved the charge transport which resulted in relatively higher PCE.

\subsubsection{Experimental}

This section includes the synthesis of $\mathrm{CuInS}_{2}$ NCs followed by the fabrication of perovskite solar cells. The solar cells were fabricated with or without $\mathrm{CuInS}_{2}$ NC layer on top of perovskite layer.

\subsubsection{Synthesis of $\mathrm{CuInS}_{2}$ NCs}

Synthesis of CuInS ${ }_{2}$ NCs were conducted using a single-pot colloidal route method (Section 1.2.5), where the precursor of copper, indium, and sulfur were heated up in the presence of suitable ligand, and solvent. NCs were purified by solvent/antisolvent technique from its growth solution (Section 1.2.5.3) [290].

Materials: 1-octadecene $90 \%$, 1-DDT (<98\%), copper (I) iodide (99.999\%, trace metal basis), indium (III) acetate ( $99.99 \%$ trace metal basis), chloroform, and acetone were purchased from Sigma-Aldrich. 

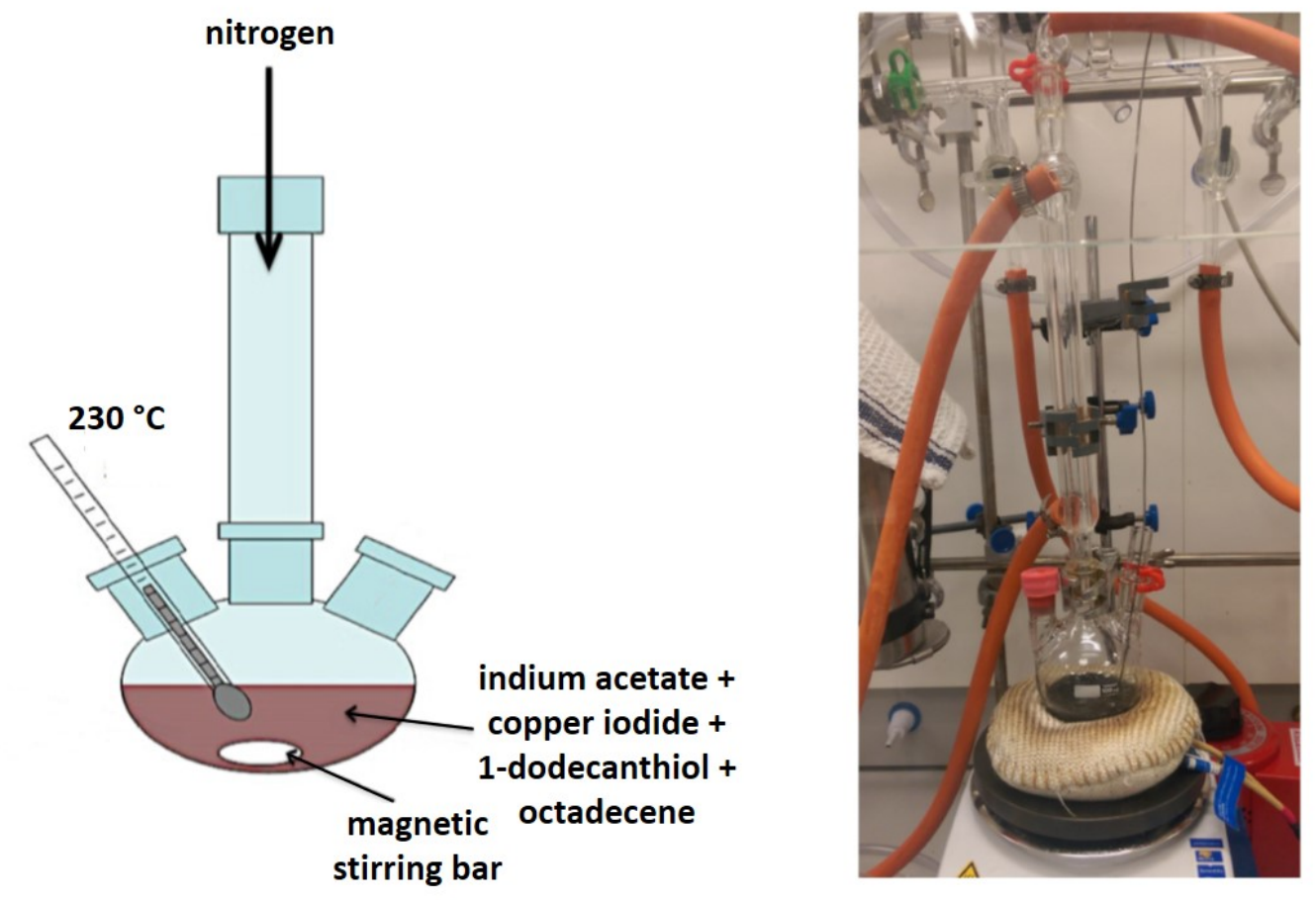

Figure 7.1: A schematic for the synthesis of CuInS $S_{2}$ NCs with a photograph of the reaction flask during the synthesis.

Methods: The CuInS 2 (CIS) NCs were prepared by typical single-pot synthesis described in 1.2.5.2, discovered by Peter Reiss, using copper(I) iodide, indium(III) acetate, 1-dodecanethiol (DDT), 1-octadecene, and oleylamine. In this method, indium acetate $(0.1 \mathrm{mmol})$ and copper iodide $(0.1 \mathrm{mmol})$ were dissolved in $8 \mathrm{~mL}$ of ODE and $1 \mathrm{~mL}$ of DDT in 3-neck $50 \mathrm{~mL}$ round bottom flask. The reaction mixture was degassed for $1 \mathrm{~h}$. After degassing the reaction solution, the temperature was raised to $230{ }^{\circ} \mathrm{C}$ under nitrogen, which resulted in change of the reaction colour from colourless to yellow, red, and then dark brown. After $40 \mathrm{~min}$, the heating source was removed and the flask was cooled down with iced-water bath. NCs growth solution was washed two times with acetone and chloroform and subsequently dispersed in anhydrous chloroform. 


\subsubsection{Fabrication of Solar Cell}

A spin coating and thermal evaporation techniques with shadow masking were used for the fabrication of devices. Fabrication of solar cells including the electrical measurements were carried out by Dr Yingzhuang Ma under the supervision of Prof Jonathan E. Halpert at Victoria University of Wellington.

Fluorine doped tin oxide (FTO, Nippon Sheet Glass, $10 \mathrm{ohmsq}^{-1}$ ) glass was cleaned sequentially in detergent, water, acetone, and isopropanol under ultrasonication for $15 \mathrm{~min}$, respectively, and then treated with $\mathrm{O}_{2}$ plasma for $3 \mathrm{~min}$. A compact $\mathrm{TiO}_{2}$ layer on the FTO glass was prepared by spin-coating of $0.15 \mathrm{M}$ titanium diisopropoxide bis(acetylacetonate) (75 wt. \% in isopropanol) solution in 1-butanol (99.8\%, Sigma-Aldrich) at $4000 \mathrm{rpm}$ for $30 \mathrm{~s}$, dried at $125^{\circ} \mathrm{C}$ for $5 \mathrm{~min}$. After that, the mesoporous $\mathrm{TiO}_{2}$ film was prepared by spin-coating a $20 \mathrm{~nm}$-sized $\mathrm{TiO}_{2}$ paste (diluted in ethanol with a ratio of 2:7 by weight, Dyesol) at $4000 \mathrm{rpm}$ for $30 \mathrm{~s}$, dried at $125^{\circ} \mathrm{C}$ for $5 \mathrm{~min}$, then heated at $500{ }^{\circ} \mathrm{C}$ for $15 \mathrm{~min}$. For the preparation of perovskite layer, $1.0 \mathrm{M} \mathrm{PbI}_{2}$ (anhydrous, $99 \%$, SigmaAldrich) in N,N-Dimethylformamide (anhydrous, $99.99 \%$, Sigma Aldrich) was spin-coated at $4000 \mathrm{rpm}$ at $70{ }^{\circ} \mathrm{C}$ for $30 \mathrm{~s}$, dried at $70{ }^{\circ} \mathrm{C}$ for $10 \mathrm{~min}$. After that, $0.5 \mathrm{~mL} \mathrm{CH}_{3} \mathrm{NH}_{3} \mathrm{I}$ (Dyesol) solution ( $8 \mathrm{mg} / \mathrm{mL}$ in isopropanol) was continuously drop-casted on $\mathrm{PbI}_{2}$ for $20 \mathrm{~s}$ at $2000 \mathrm{rpm}$ to form perovskite crystalline, and then further annealed at $90{ }^{\circ} \mathrm{C}$ for $60 \mathrm{~min}$. For the device with CIS NCs, a layer was spin-coated at $2000 \mathrm{rpm}$ for $30 \mathrm{~s}$. The HTL was then deposited by spin coating at $4000 \mathrm{rpm}$ for $45 \mathrm{~s}$. The spin-coating formulation was prepared by dissolving $72.3 \mathrm{mg}$ spiro-MeOTAD (99 \%, Lumtec), $28.8 \mu \mathrm{L}$ 4-tert-butylpyridine (96\%, Sigma-Aldrich), $17.5 \mu \mathrm{L}$ of a stock solution of $520 \mathrm{mg} / \mathrm{mL}$ lithium bis(trifluoromethylsulphonyl)imide (98\%, Sigma-Aldrich) in acetonitrile (anhydrous, 99.8 \%, Sigma-Aldrich) in $1 \mathrm{~mL}$ chlorobenzene (anhydrous, $99.8 \%$, Sigma-Aldrich). Finally, $1000 \mathrm{~nm}$ Au was thermally evaporated under vacuum [246].

Solar cells were electrically characterised using a Keithley 236 Source measurement unit and a xenon lamp based solar simulator (Sciencetech, 500-IG) was 
used to photo-excite the devices by Dr Yingzhuang Ma [246]. SEM measurements on solar cells were conducted using Jeol 6500 by Dr Yingzhuang Ma. The PCE, fill factor (FF), open circuit voltage $\left(\mathrm{V}_{o c}(\right.$, and short circuit current density $\left(\mathrm{J}_{s c}\right)$ of solar cells were calculated by method described in Section 1.5.2.

\subsubsection{Results and Discussion}

NCs were characterised by TEM (Jeol 2100) and XRD (PANalytical X-ray diffractometer) to confirm their morphology and crystal structure. The NCs solution in chloroform was drop casted on a Cu-200 mesh on formvar grid for TEM measurements. A histogram was created using ImageJ, which is placed on top of TEM image (Figure 7.2). The particle size was estimated to be $5.65 \pm 0.62 \mathrm{~nm}$. The XRD sample was prepared by drop casting concentrated solution of NCs on a glass slide. XRD spectrum shown in Figure 7.2 are compared with its standard spectrum taken from from PANalytical X'Pert HighScore Plus. This confirms the formation of $\mathrm{CuInS}_{2}$ crystals. However, the diffraction peaks were broader compared to their index spectrum according to the Scherrer equation 1.39.

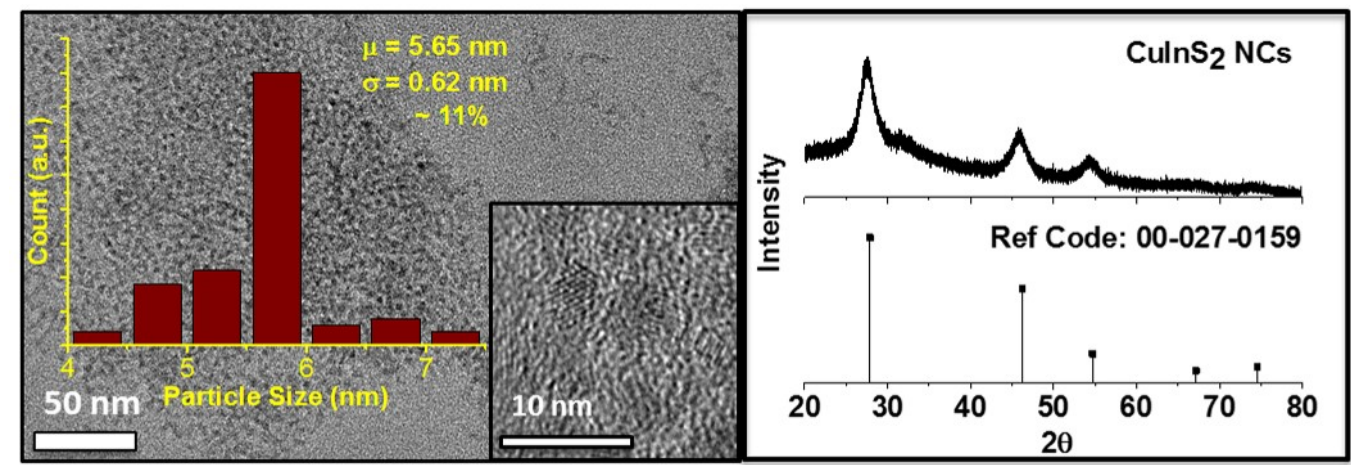

Figure 7.2: TEM micrograph of $\mathrm{CuInS}_{2}$ NCs (left) confirming the particle size of $5.65 \pm 0.62 \mathrm{~nm}$ using a histogram and XRD (right) of the same sample confirms the formation of CuInS 2 crystal structure. The standard index spectrum of $\mathrm{CuInS}_{2}$ was taken from PANalytical X'Pert HighScore Plus (Reference Code: 00027-0159). 

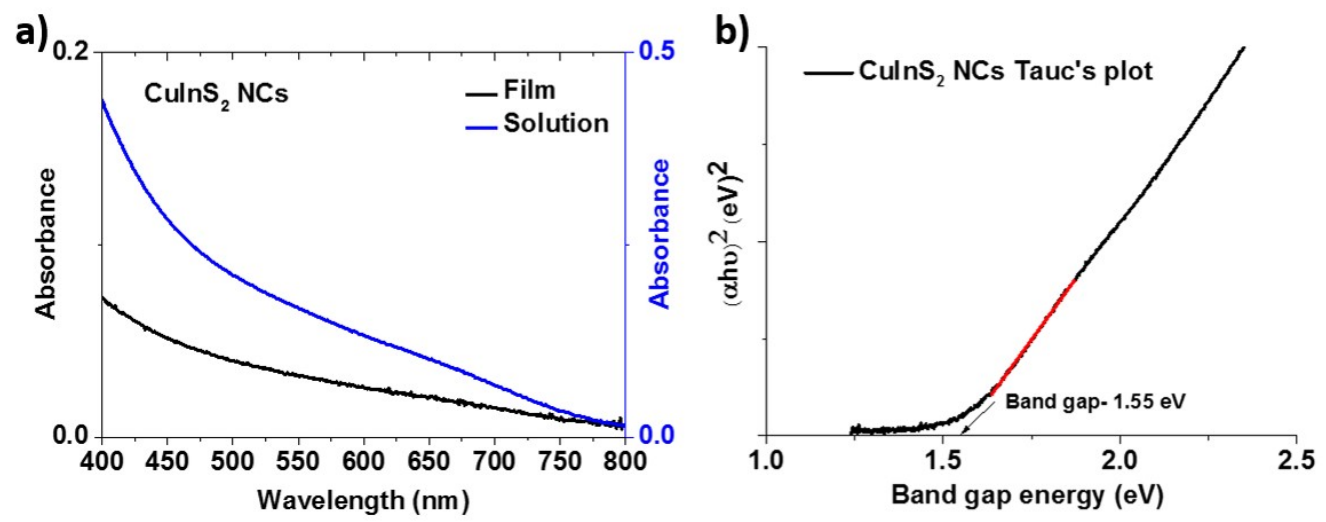

Figure 7.3: Absorption spectra of CuInS 2 NCs in chloroform solvent and in thin film on a glass substrate by Cary 50 UV-Vis spectrophotometer. All the samples were washed by solvent/antisolvent method before the measurement. b) Tauc plot of CuInS $S_{2}$ NCs for the band gap calculation. Copyright Willey Online Library 2017.

UV-Vis measurements were carried out using Cary 50 UV-Vis spectrophotometer on $\mathrm{CuInS}_{2}$ NCs solution in chloroform solvent and thin film on a glass substrate (Figure 7.3a). It can be seen that the absorption edge was found to be around $\approx 700 \mathrm{~nm}$. Figure $7.3 \mathrm{~b}$ shows the Tauc plot of $\mathrm{CuInS}_{2} \mathrm{NCs}$ for the direct band gap semiconductor, which suggest the band gap of $1.55 \mathrm{eV}$.

In order to observe the CIS NCs thin film on perovskite, SEM measurements were conducted on $\mathrm{FTO} / \mathrm{TiO}_{2} /$ perovskite and $\mathrm{FTO} / \mathrm{TiO}_{2}$ / perovskite thin films by Dr Yingzhuang Ma. Figure 7.4 shows the SEM micrographs of both the thin films. Figure 7.4 confirms the formation of perovskite cubic crystalline. It can be also seen that the CIS NCs were uniformly deposited on top of perovskite crystals.

Figure 7.5 shows the energy diagram of perovskite solar cells [246]. As can be seen that the electrons transport through the electron transport material $\mathrm{TiO}_{2}$ and holes transport through the hole transport materials $\left(\mathrm{CuInS}_{2}\right.$ and spiroMeOTAD). CuInS 2 NCs layers improve the hole transport as the valence band 


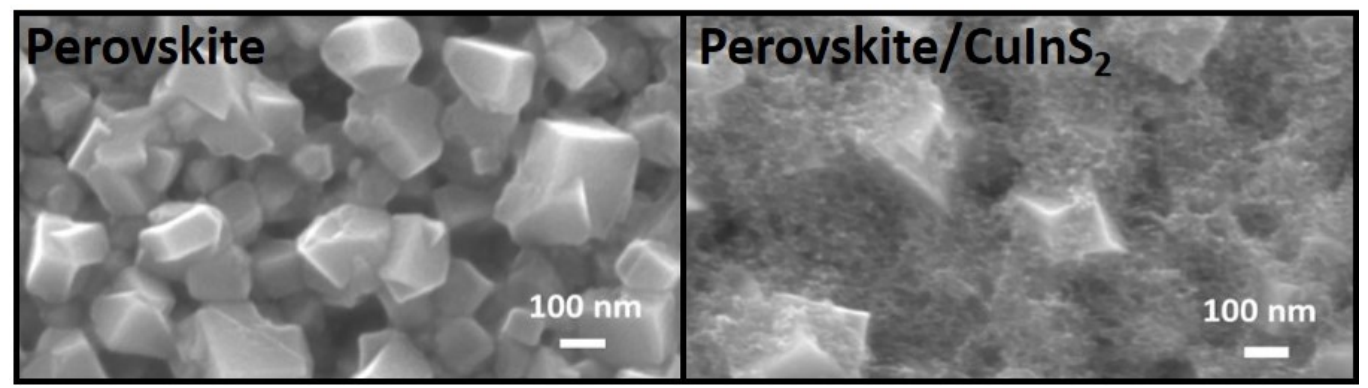

Figure 7.4: SEM measurements on $\mathrm{FTO}_{\mathrm{TiO}} /$ perovskite and $\mathrm{FTO} / \mathrm{TiO}_{2} /$ perovskite/CuInS 2 thin films. Courtesy Dr Yingzhuang Ma. Copyright Wiley Online Library

of $\mathrm{CuInS}_{2}$ NCs is very close to the valence band of spiro-MeOTAD (Figure 7.5a). This improved hole transport results in higher power conversion efficiency (PCE) of these solar cells from 12.4 to $13.8 \%$ (Table 7.1). Figure $7.5 \mathrm{c}$ and $\mathrm{d}$ shows the schematic of perovskite solar cells with and without $\mathrm{CuInS}_{2} \mathrm{NCs}$ layer. This also illustrates the another role of $\mathrm{CuInS}_{2} \mathrm{NCs}$, which is to enhance the stability against moisture. In order to check the moisture stability, fabricated solar cells were exposed to different humidity conditions for $20 \mathrm{~h}$ in a sealed box. As can be seen from Figure $7.5 \mathrm{~b}$, the perovskite solar cells with $\mathrm{CuInS}_{2}$ layer (red colour) showed higher stability against humidity. Under $90 \%$ humidity, the power conversion efficiency and fill factor of pure perovskite solar cells dropped down significantly. Whereas, devices with a layer of $\mathrm{CuInS}_{2} \mathrm{NCs}$ showed relatively less decay in the power conversion efficiency and fill factor of solar cells even after several hours (Figure 7.5a). The detailed study of PCE, $\mathrm{J}_{s c}, \mathrm{~V}_{o c}$, and FF at different humidity conditions, conducted by Dr Yingzhuang Ma, can

Table 7.1: Efficiency metrics of perovskite solar cells with and without CuInS 2 NCs layer.

\begin{tabular}{|c|c|c|c|c|}
\hline Device Structure & $\mathbf{J}_{s c}\left(\mathbf{m A} / \mathbf{c m}^{2}\right)$ & $\mathbf{V}_{o c}(\mathbf{m V})$ & FF (\%) & PCE (\%) \\
\hline No CIS & 21.8 & 1010 & 56.3 & 12.4 \\
With CIS & 21.8 & 1040 & 61.2 & 13.8 \\
\hline
\end{tabular}


a)

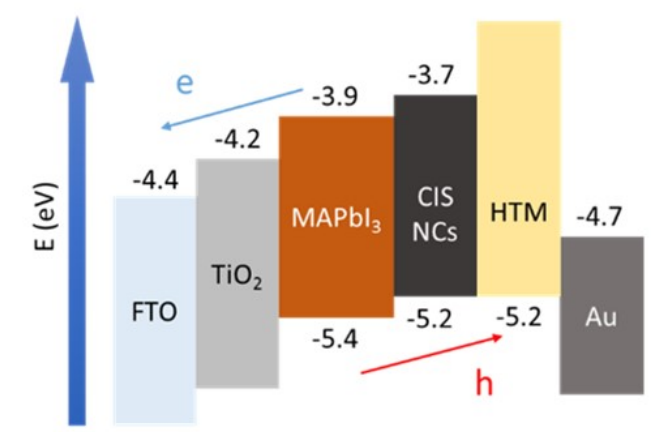

c)

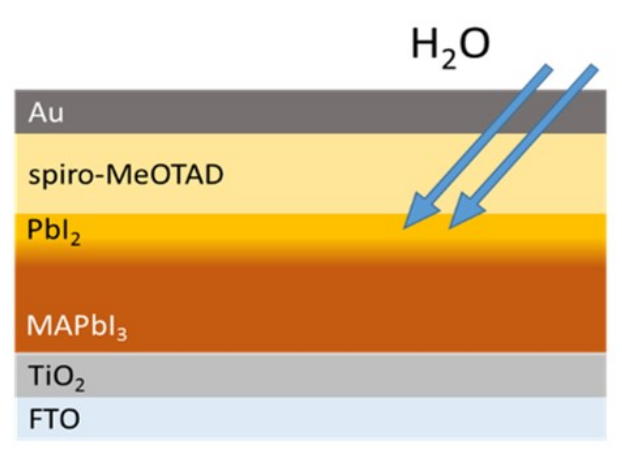

b)

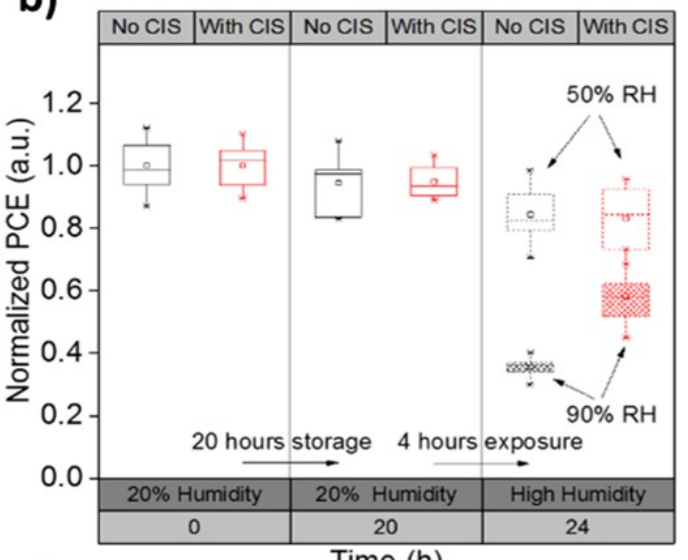

d)

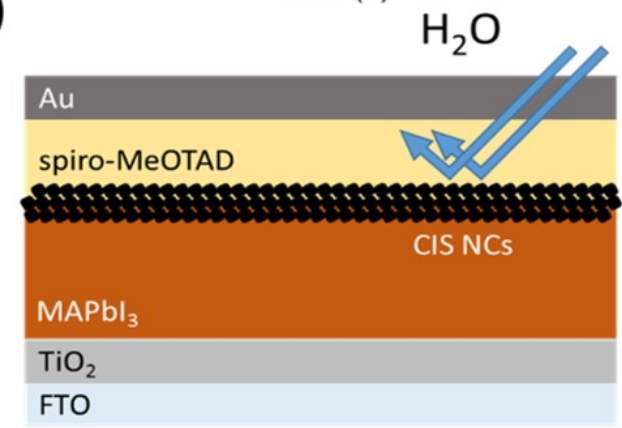

Figure 7.5: a) Energy diagram of perouskite solar cells, [246], b) a chart showing the change in PCE as a function of time and humidity for perouskite solar cells with and without CuInS $S_{2}$ NCs, c) a schematic diagram of perovskite solar cell without CuInS $S_{2} \mathrm{NCs}$, and d) the same schematic diagram of perovskite solar cells when the CuIn $S_{2}$ NCs were deposited on top of perouskite layer. The illustration shows that the CuInS $S_{2}$ surface tend to repel the water molecules (moisture) and thus make it more stable in ambient conditions. Copyright Wiley Online Library 2017. Courtesy of Dr Yingzhuang Ma.

be found in our publication [246]. 


\subsubsection{Conclusion}

We have synthesised high quality $\mathrm{CuInS}_{2}$ NCs by colloidal route with desired band gap to enhance the charge injection in perovskite solar cells. These $\mathrm{CuInS}_{2}$ NCs were used as one of the hole transport layers which resulted in improved efficiency of perovskite solar cells from 12.4 to $13.8 \%$. Humidity tests were performed with and without CuInS 2 NCs layer in perovskite solar cells, in which several humidity conditions were artificially created to observe the performance of these devices. Perovskite solar cells with $\mathrm{CuInS}_{2}$ NCs layer were found to have higher resistance towards the moisture and even at higher humidity of 90 $\%$, the stability of these devices were found to be significantly improved. This method of perovskite film protection with $\mathrm{CuInS}_{2}$ NCs layer is a promising way to increase the device stability and performance.

\subsection{Appendix 2- $\mathrm{Er}^{3+}$ Doping in $\mathrm{Tl}_{3} \mathrm{PbBr}_{5} \mathrm{NCs}$}

$\mathrm{Tl}_{3} \mathrm{PbBr}_{5}$ NCs were synthesised by a colloidal route in Chapter 5 . These NCs are potential candidates for non-linear optics when doped with rare earth materials, such as $\mathrm{Er}^{3+}$ and $\operatorname{Pr}^{3+}[252,253,256]$. Therefore, We have gained focus towards doping $\mathrm{Tl}_{3} \mathrm{PbBr}_{5} \mathrm{NCs}$ with $\mathrm{Er}^{3+}$. A similar hot-injection method was used as described in Section 5.2.2 with slight modifications.

\subsubsection{Synthesis of $\mathrm{Er}^{3+}$-doped $\mathrm{Tl}_{3} \mathrm{PbBr}_{5} \mathrm{NCs}$}

This method is divided into two parts, synthesis of thallium oleate precusor followed by the synthesis of NCs. Thallium oleate was synthesised by loading $0.46 \mathrm{gm}$ of thallium(I) acetate (Aldrich), $15 \mathrm{~mL}$ of 1-octadecene (Aldrich), and $1.6 \mathrm{~mL}$ of oleic acid (Aldrich) into a $50 \mathrm{~mL}$ of 3-neck round bottom flask. The reaction mixture was degassed under vacuum at $120^{\circ} \mathrm{C}$ for $1 \mathrm{~h}$. Then, the tem- 
perature was raised to $150{ }^{\circ} \mathrm{C}$ for next $30 \mathrm{~min}$ and the reaction was switched to $\mathrm{N}_{2}$ from vacuum. The final pale yellow color solution was the thallium oleate, which was transferred to a Schlenk tube and stored in $\mathrm{N}_{2}$ glove box until it was required for the synthesis of NCs.

A $0.38 \mathrm{mmol}$ portion of $\mathrm{PbBr}_{2}$ (Aldrich) was loaded in to a $50 \mathrm{~mL}$ 3-neck round bottom flask along with $10 \mathrm{~mL}$ of 1-octadecene. The reaction solution was degassed under vacuum at $120^{\circ} \mathrm{C}$ for $1 \mathrm{~h}$. Simultaneously, $1.0 \mathrm{~mL}$ oleylamine and oleic acid were also degassed under the same conditions in two separate vials. After degassing, oleylamine, oleic acid, and $20 \mathrm{mg}$ of Er(III) fluoride (Aldrich) were added into the reaction flask under $\mathrm{N}_{2}$, and the temperature was raised to $175{ }^{\circ} \mathrm{C}$. After this, $1.1 \mathrm{~mL}$ of thallium oleate was quickly injected into the reaction flask. After $45 \mathrm{~s}$ the reaction flask was cooled down using an ice bath.

NCs were purified by solvent/antisolvent method (Section 1.2.5.3). The growth solution was centrifuged at 10,000 rpm for $10 \mathrm{~min}$. After that, the supernatant was discarded, and the precipitate was redispersed in $6 \mathrm{~mL}$ of toluene (solvent) with $3 \mathrm{~mL}$ of acetonitrile (antisolvent) and centrifuged again at 10,000 rpm for $10 \mathrm{~min}$. After discarding the supernatant, the precipitate was dried under vacuum for $5 \mathrm{~min}$ and then redispersed in $10 \mathrm{~mL}$ of hexane. The purification process was conducted in a nitrogen glove box due to the high toxicity of thallium.

\subsubsection{Results and Discussion}

NCs were characterised by UV-Vis and PL measurements to analyse the optical properties. In addition, TEM was used to observe the morphology and erbium doping in NCs. Each NC samples were diluted in hexane for the UV-Vis and PL measurements and a $10 \mathrm{~mm}$ quartz cuvette with pure hexane was used to create the baseline. A Cary 50 UV-Visible spectrophotometer was used to conduct the UV-Vis measurements and a Horiba Fluorolog-3 Spectrometer was used for the PL measurements. The TEM samples were prepared by drop-casting hexane 

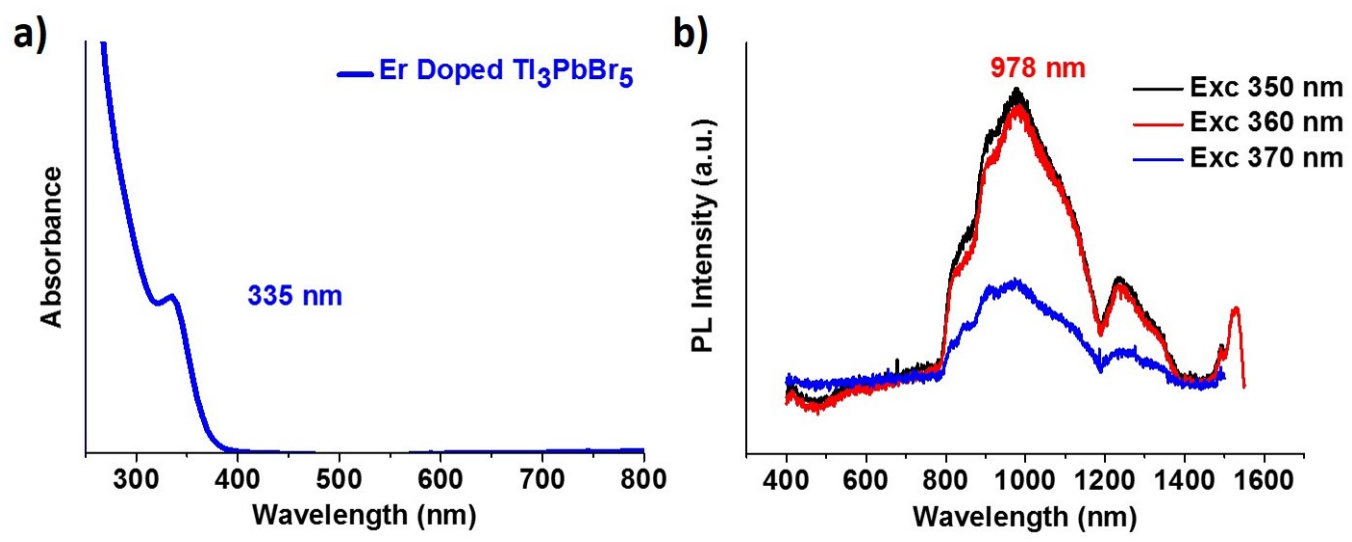

Figure 7.6: a) Absorption and b) emission spectrum of $\mathrm{Er}^{3+}$-doped $\mathrm{Tl}_{3} \mathrm{PbBr}_{5} \mathrm{NCs}$. NCs were diluted in hexane for the measurements.

NC solution on a Cu-200 mesh on formvar grid.

UV -Vis measurement shows the first absorption edge at $335 \mathrm{~nm}$ (Figure 7.6a), which is consistent with the absorption spectra observed in Figure 5.12. PL
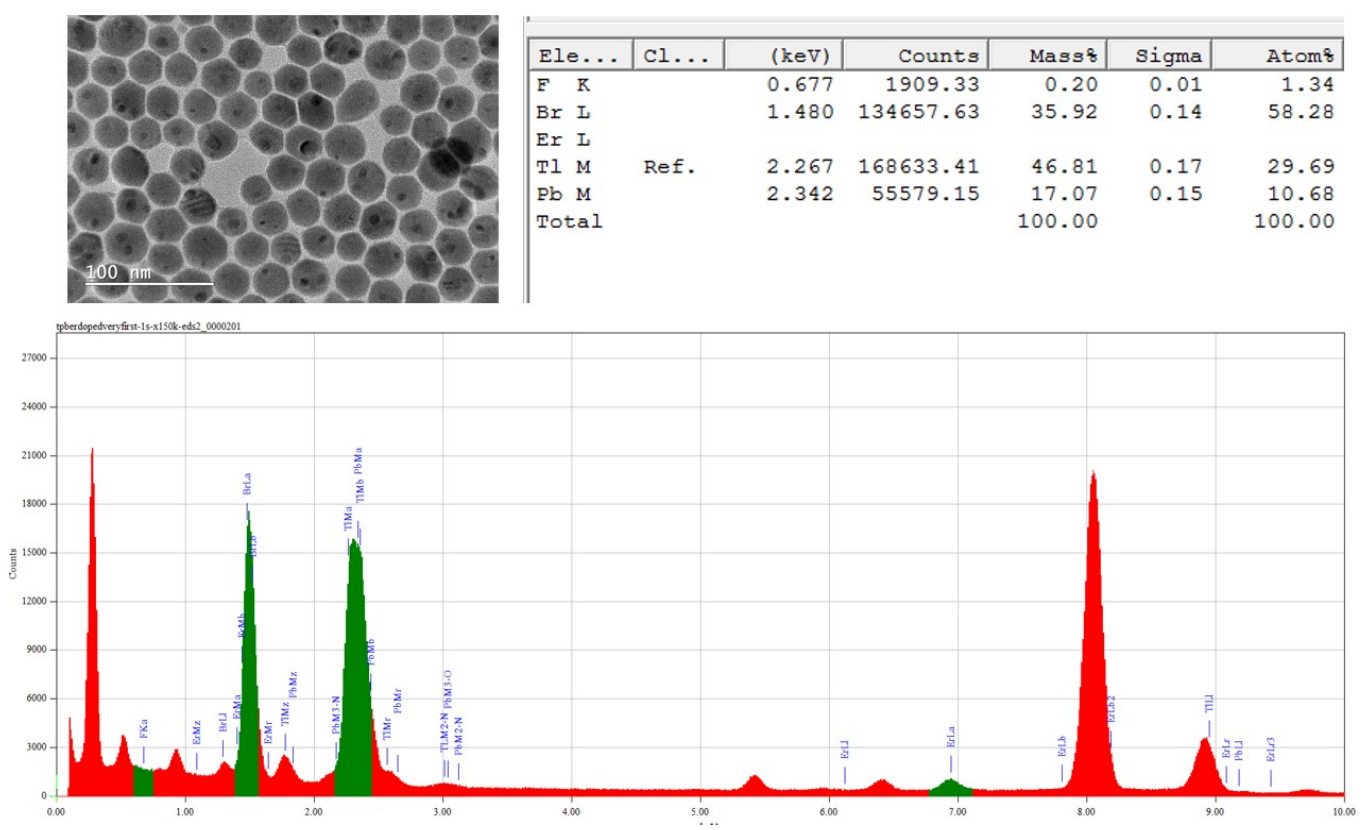

Figure 7.7: TEM analysis of $\mathrm{Er}^{3+}$-doped $\mathrm{Tl}_{3} \mathrm{PbBr}_{5} \mathrm{NCs}$. Samples were prepared by drop-casting hexane NCs solution on a Cu-200 mesh on formvar grid. 
measurement was carried out at different excitation wavelength and a broad band emission at $\approx 958 \mathrm{~nm}$ was observed (Figure 7.6). Emission between 750900 is the characteristic emission from $\mathrm{Er}^{3+}$ [291]. This emission study suggests the doping of $\mathrm{Er}^{3+}$ in $\mathrm{Tl}_{3} \mathrm{PbBr}_{5}$ NCs. However, a detailed spectroscopy analysis in required to confirm the doping.

Figure 7.7 shows the TEM image of $\mathrm{Er}^{3+}$-doped $\mathrm{Tl}_{3} \mathrm{PbBr}_{5} \mathrm{NCs}$, which displays faceted spheroidal shape with $\approx 29 \mathrm{~nm}$ particle diameter. The EDS analysis shows the incorporation of $\mathrm{Er}^{3+}$ into the NCs. However, the amount of erbium was too less to be observed quantitatively. A detailed analysis is still needed to analyse the doping amount.

\subsection{Conclusion}

We have synthesised $\mathrm{Er}^{3+}$ - doped $\mathrm{Tl}_{3} \mathrm{PbBr}_{5} \mathrm{NCs}$ by a colloidal route. NCs were optically characterised to observe the emission from $\mathrm{Er}^{3+}$. PL measurements showed the broad band emission in near IR spectral region, which suggested the doping of $\mathrm{Er}^{3+}$ into the NCs. TEM EDS also showed a low intensity erbium peak. However, further work is still required in this project, which is the part of future research in Halpert Group. 


\section{Bibliography}

[1] Polina O Anikeeva, Jonathan E Halpert, Moungi G Bawendi, and Vladimir Bulovic. Quantum dot light-emitting devices with electroluminescence tunable over the entire visible spectrum. Nano Letters, 9(7):2532-2536, 2009.

[2] Yixing Yang, Ying Zheng, Weiran Cao, Alexandre Titov, Jake Hyvonen, Jesse R Manders, Jiangeng Xue, Paul H Holloway, and Lei Qian. Highefficiency light-emitting devices based on quantum dots with tailored nanostructures. Nature Photonics, 9(4):259-266, 2015.

[3] Yasuhiro Shirasaki, Geoffrey J Supran, William A Tisdale, and Vladimir Bulović. Origin of efficiency roll-off in colloidal quantum-dot lightemitting diodes. Physical review letters, 110(21):217403, 2013.

[4] Igor Coropceanu, Aurelio Rossinelli, Justin R Caram, Francesca S Freyria, and Moungi G Bawendi. Slow-injection growth of seeded CdSe/CdS nanorods with unity fluorescence quantum yield and complete shell to core energy transfer. ACS nano, 10(3):3295-3301, 2016.

[5] AJ Nozik. Quantum dot solar cells. Physica E: Low-dimensional Systems and Nanostructures, 14(1-2):115-120, 2002.

[6] Michael Quinten. Optical properties of nanoparticle systems: Mie and beyond. John Wiley \& Sons, 2010. 
[7] Andrew M Smith and Shuming Nie. Semiconductor nanocrystals: structure, properties, and band gap engineering. Accounts of chemical research, 43(2):190-200, 2009.

[8] Parth Vashishtha, Dani Z Metin, Matthew E Cryer, Kai Chen, Justin M Hodgkiss, Nicola Gaston, and Jonathan E Halpert. Shape-, size-, and composition-controlled thallium lead halide perovskite nanowires and nanocrystals with tuneable band gaps. Chemistry of Materials, 2018.

[9] Xiaogang Peng, Liberato Manna, Weidong Yang, Juanita Wickham, Erik Scher, Andreas Kadavanich, and A Paul Alivisatos. Shape control of CdSe nanocrystals.

[10] Wenting Shang, Xinchen Kang, Hui Ning, Jianling Zhang, Xiaogang Zhang, Zhonghua Wu, Guang Mo, Xueqing Xing, and Buxing Han. Shape and size controlled synthesis of MOF nanocrystals with the assistance of ionic liquid mircoemulsions. Langmuir, 29(43):13168-13174, 2013.

[11] Xingliang Dai, Zhenxing Zhang, Yizheng Jin, Yuan Niu, Hujia Cao, Xiaoyong Liang, Liwei Chen, Jianpu Wang, and Xiaogang Peng. Solutionprocessed, high-performance light-emitting diodes based on quantum dots. Nature, 515(7525):96-99, 2014.

[12] Hui-Seon Kim, Chang-Ryul Lee, Jeong-Hyeok Im, Ki-Beom Lee, Thomas Moehl, Arianna Marchioro, Soo-Jin Moon, Robin Humphry-Baker, JunHo Yum, Jacques E Moser, et al. Lead iodide perovskite sensitized allsolid-state submicron thin film mesoscopic solar cell with efficiency exceeding 9\%. Scientific reports, 2:591, 2012.

[13] Qingbo Zhang, Jianping Xie, Yue Yu, and Jim Yang Lee. Monodispersity control in the synthesis of monometallic and bimetallic quasi-spherical gold and silver nanoparticles. Nanoscale, 2(10):1962-1975, 2010.

[14] Lei Meng, En-Ping Yao, Ziruo Hong, Huajun Chen, Pengyu Sun, Zhanlue Yang, Gang Li, and Yang Yang. Pure formamidinium-based perovskite 
light-emitting diodes with high efficiency and low driving voltage. Advanced Materials, 29(4), 2017.

[15] Yingzhuang Ma, Parth Vashishtha, Kai Chen, Elijah L Peach, David Ohayon, Justin M Hodgkiss, and Jonathan E Halpert. Controlled growth of $\mathrm{CH}_{3} \mathrm{NH}_{3} \mathrm{PbI}_{3}$ using a dynamically dispensed spin-coating method: improving efficiency with a reproducible $\mathrm{PbI}_{2}$ blocking layer. ChemSusChem, 2017.

[16] Haiming Zhu, Yongping Fu, Fei Meng, Xiaoxi Wu, Zizhou Gong, Qi Ding, Martin V Gustafsson, M Tuan Trinh, Song Jin, and XY Zhu. Lead halide perovskite nanowire lasers with low lasing thresholds and high quality factors. Nature materials, 14(6):636, 2015.

[17] Loredana Protesescu, Sergii Yakunin, Maryna I Bodnarchuk, Franziska Krieg, Riccarda Caputo, Christopher H Hendon, Ruo Xi Yang, Aron Walsh, and Maksym V Kovalenko. Nanocrystals of cesium lead halide perovskites ( $\mathrm{CsPbX}, \mathrm{X}=\mathrm{Cl}, \mathrm{Br}$, and I): Novel optoelectronic materials showing bright emission with wide color gamut. Nano Letters, 2015.

[18] Saikat Bhaumik, Sjoerd A Veldhuis, Yan Fong Ng, Mingjie Li, Subas Kumar Muduli, Tze Chien Sum, Bahulayan Damodaran, Subodh Mhaisalkar, and Nripan Mathews. Highly stable, luminescent core-shell type methylammonium-octylammonium lead bromide layered perovskite nanoparticles. Chemical Communications, 52(44):7118-7121, 2016.

[19] Duong Nguyen Minh, Juwon Kim, Jinho Hyon, Jae Hyun Sim, Haneen H Sowlih, Chunhee Seo, Jihye Nam, Sangwon Eom, Soyeon Suk, and Sangheon Lee. Room-temperature synthesis of widely runable formamidinium lead halide perovskite nanocrystals. Chemistry of Materials, 29(13):5713-5719, 2017.

[20] Parth Vashishtha and Jonathan E Halpert. Field-driven ion migration and 
color instability in red-emitting mixed halide perovskite nanocrystal light emitting diodes. Chemistry of Materials, 29(14):5965-5973, 2017.

[21] Jizhong Song, Jianhai Li, Xiaoming Li, Leimeng Xu, Yuhui Dong, and Haibo Zeng. Quantum dot light-emitting diodes based on inorganic perovskite cesium lead halides $\left(\mathrm{CsPbX}_{3}\right)$. Advanced Materials, 27(44):71627167, 2015.

[22] Tom C Jellicoe, Johannes M Richter, Hugh FJ Glass, Maxim Tabachnyk, Ryan Brady, Siaİ́n E Dutton, Akshay Rao, Richard H Friend, Dan Credgington, and Neil C Greenham. Synthesis and optical properties of leadfree cesium tin halide perovskite nanocrystals. Journal of the American Chemical Society, 138(9):2941-2944, 2016.

[23] Bin Yang, Junsheng Chen, Feng Hong, Xin Mao, Kaibo Zheng, Songqiu Yang, Yajuan Li, Tonu Pullerits, Weiqiao Deng, and Keli Han. Lead-free, air-stable all-inorganic cesium bismuth halide perovskite nanocrystals. Angewandte Chemie International Edition, pages 12471-12475.

[24] Sidney E Creutz, Evan N Crites, Michael C De Siena, and Daniel R Gamelin. Colloidal nanocrystals of lead-free double-perovskite (elpasolite) semiconductors: synthesis and anion exchange to access new materials. Nano letters, 18(2):1118-1123, 2018.

[25] Bin Yang, Junsheng Chen, Songqiu Yang, Feng Hong, Lei Sun, Peigeng Han, Tõnu Pullerits, Weiqiao Deng, and Keli Han. Lead-free silverbismuth halide double perovskite nanocrystals. Angewandte Chemie, 2018.

[26] DS Ginger and NC Greenham. Charge injection and transport in films of CdSe nanocrystals. Journal of Applied Physics, 87(3):1361-1368, 2000.

[27] Jiang Tang, Kyle W Kemp, Sjoerd Hoogland, Kwang S Jeong, Huan Liu, Larissa Levina, Melissa Furukawa, Xihua Wang, Ratan Debnath, Dongkyu 
Cha, et al. Colloidal-quantum-dot photovoltaics using atomic-ligand passivation. Nature materials, 10(10):765, 2011.

[28] Yuequn Shang, Gang Li, Weimin Liu, and Zhijun Ning. Quasi-2D inorganic $\mathrm{CsPbBr}_{3}$ perovskite for efficient and stable light-emitting diodes. Advanced Functional Materials, page 1801193, 2018.

[29] Li Na Quan, Yongbiao Zhao, F Pelayo Garcia de Arquer, Randy Sabatini, Grant Walters, Oleksandr Voznyy, Riccardo Comin, Yiying Li, James Z Fan, Hairen Tan, et al. Tailoring the Energy Landscape in Quasi-2D Halide Perovskites Enables Efficient Green-Light Emission. Nano letters, 17(6):3701-3709, 2017.

[30] Junjie Si, Yang Liu, Zhuofei He, Hui Du, Kai Du, Dong Chen, Jing Li, Mengmeng Xu, He Tian, Haiping He, et al. Efficient and high-color-purity light-emitting diodes based on in situ grown films of $\mathrm{CsPbX}_{3}(\mathrm{X}=\mathrm{Br}, \mathrm{I})$ nanoplates with controlled thicknesses. ACS nano, 11(11):11100-11107, 2017.

[31] Nana Wang, Lu Cheng, Rui Ge, Shuting Zhang, Yanfeng Miao, Wei Zou, Chang Yi, Yan Sun, Yu Cao, Rong Yang, et al. Perovskite light-emitting diodes based on solution-processed self-organized multiple quantum wells. Nature Photonics, 10(11):699, 2016.

[32] Zhengguo Xiao, Ross A Kerner, Lianfeng Zhao, Nhu L Tran, Kyung Min Lee, Tae-Wook Koh, Gregory D Scholes, and Barry P Rand. Efficient perovskite light-emitting diodes featuring nanometre-sized crystallites. $\mathrm{Na}$ ture Photonics, 11(2):108, 2017.

[33] Xiaolei Yang, Xingwang Zhang, Jinxiang Deng, Zema Chu, Qi Jiang, Junhua Meng, Pengyang Wang, Liuqi Zhang, Zhigang Yin, and Jingbi You. Efficient green light-emitting diodes based on quasi-two-dimensional composition and phase engineered perovskite with surface passivation. Nature communications, 9(1):570, 2018. 
[34] Himchan Cho, Su-Hun Jeong, Min-Ho Park, Young-Hoon Kim, Christoph Wolf, Chang-Lyoul Lee, Jin Hyuck Heo, Aditya Sadhanala, NoSoung Myoung, Seunghyup Yoo, et al. Overcoming the electroluminescence efficiency limitations of perovskite light-emitting diodes. Science, 350(6265):1222-1225, 2015.

[35] Mingjian Yuan, Li Na Quan, Riccardo Comin, Grant Walters, Randy Sabatini, Oleksandr Voznyy, Sjoerd Hoogland, Yongbiao Zhao, Eric M Beauregard, and Pongsakorn Kanjanaboos. Perovskite energy funnels for efficient light-emitting diodes. Nature nanotechnology, 11(10):872-877, 2016.

[36] Junxue Liu, Jing Leng, Kaifeng Wu, Jun Zhang, and Shengye Jin. Observation of internal photoinduced electron and hole separation in hybrid two-dimentional perovskite films. Journal of the American Chemical Society, 139(4):1432-1435, 2017.

[37] María C Gélvez-Rueda, Eline M Hutter, Duyen H Cao, Nicolas Renaud, Constantinos C Stoumpos, Joseph T Hupp, Tom J Savenije, Mercouri G Kanatzidis, and Ferdinand C Grozema. Interconversion between free charges and bound excitons in 2D hybrid lead halide perovskites. The Journal of Physical Chemistry C, 121(47):26566-26574, 2017.

[38] Tomas Leijtens, Samuel D Stranks, Giles E Eperon, Rebecka Lindblad, Erik MJ Johansson, Ian J McPherson, Hakan Rensmo, James M Ball, Michael M Lee, and Henry J Snaith. Electronic properties of mesosuperstructured and planar organometal halide perovskite films: charge trapping, photodoping, and carrier mobility. ACS nano, 8(7):7147-7155, 2014.

[39] Lu Cheng, Yu Cao, Rui Ge, Ying-Qiang Wei, Na-Na Wang, Jian-Pu Wang, and Wei Huang. Sky-blue perovskite light-emitting diodes based on quasi-two-dimensional layered perovskites. Chinese Chemical Letters, 28(1):29-31, 2017. 
[40] AI Ekimov and AA Onushchenko. Quantum size effect in threedimensional microscopic semiconductor crystals. ZhETF Pisma Redaktsiiu, 34:363, 1981.

[41] R Rossetti, S Nakahara, and Louis E Brus. Quantum size effects in the redox potentials, resonance Raman spectra, and electronic spectra of CdS crystallites in aqueous solution. The Journal of Chemical Physics, 79(2):1086-1088, 1983.

[42] Louis E Brus. Electron-electron and electron-hole interactions in small semiconductor crystallites: The size dependence of the lowest excited electronic state. The Journal of chemical physics, 80(9):4403-4409, 1984.

[43] R Rossetti, JL Ellison, JM Gibson, and Louis E Brus. Size effects in the excited electronic states of small colloidal CdS crystallites. The Journal of chemical physics, 80(9):4464-4469, 1984.

[44] R Rossetti, R Hull, JM Gibson, and Louis E Brus. Excited electronic states and optical spectra of ZnS and CdS crystallites in the 15 to $50 \AA$ size range: Evolution from molecular to bulk semiconducting properties. The Journal of chemical physics, 82(1):552-559, 1985.

[45] Kuppuswamy Kalyanasundaram, Enrico Borgarello, Dung Duonghong, and Michael Grätzel. Cleavage of Water by Visible-Light Irradiation of Colloidal CdS Solutions; Inhibition of Photocorrosion by $\mathrm{RuO}_{2}$. Angewandte Chemie International Edition in English, 20(11):987-988, 1981.

[46] R Rossetti and L Brus. Electron-hole recombination emission as a probe of surface chemistry in aqueous cadmium sulfide colloids. The Journal of Physical Chemistry, 86(23):4470-4472, 1982.

[47] T Dannhauser, M O'neil, K Johansson, D Whitten, and G McLendon. Photophysics of quantized colloidal semiconductors. Dramatic luminescence enhancement by binding of simple amines. The Journal of Physical Chemistry, 90(23):6074-6076, 1986. 
[48] MT Nenadovic, T Rajh, and OI Micic. Size quantization in small semiconductor particles. The Journal of Physical Chemistry, 89(3):397-399, 1985.

[49] Christopher M Evans, Laura C Cass, Kathryn E Knowles, Daniel B Tice, Robert PH Chang, and Emily A Weiss. Review of the synthesis and properties of colloidal quantum dots: the evolving role of coordinating surface ligands. Journal of Coordination Chemistry, 65(13):2391-2414, 2012.

[50] N Chestnoy, R Hull, and LE Brus. Higher excited electronic states in clusters of ZnSe, CdSe, and ZnS: Spin-orbit, vibronic, and relaxation phenomena. The Journal of chemical physics, 85(4):2237-2242, 1986.

[51] Limin Qi, Jiming Ma, Humin Cheng, and Zhenguo Zhao. Reverse micelle based formation of $\mathrm{BaCO}_{3}$ nanowires. The Journal of Physical Chemistry B, 101(18):3460-3463, 1997.

[52] Mauricio Meyer, Christer Wallberg, Kazue Kurihara, and Janos H Fendler. Photosensitized charge separation and hydrogen production in reversed micelle entrapped platinized colloidal cadmium sulphide. Journal of the Chemical Society, Chemical Communications, (2):90-91, 1984.

[53] Michael L Steigerwald, A Paul Alivisatos, JM Gibson, TD Harris, R Kortan, AJ Muller, AM Thayer, TM Duncan, DC Douglass, and Louis E Brus. Surface derivatization and isolation of semiconductor cluster molecules. Journal of the American Chemical Society, 110(10):3046-3050, 1988.

[54] AR Kortan, R Hull, Robert L Opila, Moungi G Bawendi, Michael L Steigerwald, PJ Carroll, and Louis E Brus. Nucleation and growth of cadmium selendie on zinc sulfide quantum crystallite seeds, and vice versa, in inverse micelle media. Journal of the American Chemical Society, 112(4):1327-1332, 1990.

[55] Matthew A Marcus, W Flood, Michael Stiegerwald, Louis Brus, and Moungi Bawendi. Structure of capped cadmium selenide clusters by exafs. The Journal of Physical Chemistry, 95(4):1572-1576, 1991. 
[56] Ying Wang and Norman Herron. Optical properties of cadmium sulfide and lead (II) sulfide clusters encapsulated in zeolites. Journal of Physical Chemistry, 91(2):257-260, 1987.

[57] A Eychmüller, A Mews, and $\mathrm{H}$ Weller. A quantum dot quantum well: CdS/HgS/CdS. Chemical physics letters, 208(1-2):59-62, 1993.

[58] Horst Weller, U Koch, M Gutierrez, and Arnim Henglein. Photochemistry of colloidal metal sulfides. 7. Absorption and fluorescence of extremely small ZnS particles (the world of the neglected dimensions). Berichte der Bunsengesellschaft für physikalische Chemie, 88(7):649-656, 1984.

[59] Jeremy J Ramsden and Michael Grätzel. Photoluminescence of small cadmium sulphide particles. Journal of the Chemical Society, Faraday Transactions 1: Physical Chemistry in Condensed Phases, 80(4):919-933, 1984.

[60] Panagiotis Lianos and J Kerry Thomas. Cadmium sulfide of small dimensions produced in inverted micelles. Chemical Physics Letters, 125(3):299-302, 1986.

[61] ML Steigerwald and CR Sprinkle. Application of phosphine tellurides to the preparation of group II-VI (2-16) semiconductor materials. Organometallics, 7(1):245-246, 1988.

[62] JG Brennan, T Siegrist, SM Stuczynski, and ML Steigerwald. The transition from molecules to solids: molecular syntheses of $\mathrm{Ni}_{9} \mathrm{Te}_{6}\left(\mathrm{PEt}_{3}\right)_{8}$, $\mathrm{Ni}_{20} \mathrm{Te}_{18}\left(\mathrm{PEt}_{3}\right)_{12}$ and NiTe. Journal of the American Chemical Society, 111(26):9240-9241, 1989.

[63] ML Steigerwald, T Siegrist, and SM Stuczynski. Initial stages in the molecule-based growth of the solid-state compound cobalt telluride (CoTe). Inorganic Chemistry, 30(26):4940-4945, 1991. 
[64] SM Stuczynski, Y-U Kwon, and ML Steigerwald. The use of phosphine chalcogenides in the preparation of cobalt chalcogenides. Journal of organometallic chemistry, 449(1-2):167-172, 1993.

[65] Michael L Steigerwald and CE Rice. Organometallic synthesis of manganese telluride. Isolation and characterization of $\left[\left(\mathrm{Et}_{3} \mathrm{P}\right)_{2}(\mathrm{CO})_{3} \mathrm{MnTe}\right]_{2}$. Journal of the American Chemical Society, 110(13):4228-4231, 1988.

[66] JG Brennan, T Siegrist, SM Stuczynski, and ML Steigerwald. Cluster intermediates in an organometallic synthesis of palladium telluride PdTe. Journal of the American Chemical Society, 112(25):9233-9236, 1990.

[67] ML Steigerwald. Selective syntheses of iron monotelluride and iron ditelluride from organometallic precursors. Synthesis and pyrolysis of $\left[\mathrm{Cp}\left(\mathrm{Et}_{3} \mathrm{P}\right)(\mathrm{CO}) \mathrm{Fe}\right]_{2}(\mathrm{Te})_{n}$. Chemistry of Materials, 1(1):52-57, 1989.

[68] Victor F Puntes, Daniela Zanchet, Can K Erdonmez, and A Paul Alivisatos. Synthesis of hcp-Co nanodisks. Journal of the American Chemical Society, 124(43):12874-12880, 2002.

[69] Margaret A Hines and Gregory D Scholes. Colloidal PbS nanocrystals with size-tunable near-infrared emission: observation of post-synthesis self-narrowing of the particle size distribution. Advanced Materials, 15(21):1844-1849, 2003.

[70] Yadong Yin and A Paul Alivisatos. Colloidal nanocrystal synthesis and the organic-inorganic interface. Nature, 437(7059):664, 2004.

[71] A Raymond, JL Robert, and C Bernard. The electron effective mass in heavily doped GaAs. Journal of Physics C: Solid State Physics, 12(12):2289, 1979.

[72] Christophe Delerue and Michel Lannoo. Nanostructures: theory and modelling. Springer Science and Business Media, 2004. 
[73] Samuel D Stranks, Giles E Eperon, Giulia Grancini, Christopher Menelaou, Marcelo JP Alcocer, Tomas Leijtens, Laura M Herz, Annamaria Petrozza, and Henry J Snaith. Electron-hole diffusion lengths exceeding 1 micrometer in an organometal trihalide perovskite absorber. Science, 342(6156):341-344, 2013.

[74] Liang-shi Li, Jiangtao Hu, Weidong Yang, and A Paul Alivisatos. Band gap variation of size-and shape-controlled colloidal CdSe quantum rods. Nano Letters, 1(7):349-351, 2001.

[75] Mickael D Tessier, Dorian Dupont, Kim De Nolf, Jonathan De Roo, and Zeger Hens. Economic and size-tunable synthesis of InP/ZnE (E= S, Se) colloidal quantum dots. Chemistry of Materials, 27(13):4893-4898, 2015.

[76] Celso de Mello Donega. Synthesis and properties of colloidal heteronanocrystals. Chemical Society Reviews, 40(3):1512-1546, 2011.

[77] Xinhua Zhong and Yaoyu Feng. New strategy for band-gap tuning in semiconductor nanocrystals. Research on Chemical Intermediates, 34(23):287-298, 2008.

[78] Victor K LaMer and Robert H Dinegar. Theory, production and mechanism of formation of monodispersed hydrosols. Journal of the American Chemical Society, 72(11):4847-4854, 1950.

[79] Paul Mushonga, Martin O Onani, Abram M Madiehe, and Mervin Meyer. Indium phosphide-based semiconductor nanocrystals and their applications. Journal of Nanomaterials, 2012:12, 2012.

[80] Hans-Rudolf Wenk and Andrey Bulakh. Minerals: their constitution and origin. Cambridge University Press, 2016.

[81] Giles E Eperon, Victor M Burlakov, Pablo Docampo, Alain Goriely, and Henry J Snaith. Morphological control for high performance, solutionprocessed planar heterojunction perovskite solar ells. Advanced Functional Materials, 24(1):151-157, 2014. 
[82] Guichuan Xing, Nripan Mathews, Shuangyong Sun, Swee Sien Lim, Yeng Ming Lam, Michael GrÃd'tzel, Subodh Mhaisalkar, and Tze Chien Sum. Long-range balanced electron-and hole-transport lengths in organic-inorganic $\mathrm{CH}_{3} \mathrm{NH}_{3} \mathrm{PbI}_{3}$. Science, 342(6156):344-347, 2013.

[83] Guichuan Xing, Nripan Mathews, Swee Sien Lim, Natalia Yantara, Xinfeng Liu, Dharani Sabba, Michael GrÃd'tzel, Subodh Mhaisalkar, and Tze Chien Sum. Low-temperature solution-processed wavelengthtunable perovskites for lasing. Nature Materials, 13(5):476-480, 2014.

[84] Woon Seok Yang, Jun Hong Noh, Nam Joong Jeon, Young Chan Kim, Seungchan Ryu, Jangwon Seo, and Sang Il Seok. High-performance photovoltaic perovskite layers fabricated through intramolecular exchange. Science, 348(6240):1234-1237, 2015.

[85] Oscar A Jaramillo-Quintero, Rafael S Sanchez, Marina Rincon, and Ivan Mora-Sero. Bright visible-infrared light emitting diodes based on hybrid halide perovskite with Spiro-OMeTAD as a hole-injecting layer. The journal of physical chemistry letters, 6(10):1883-1890, 2015.

[86] Himchan Cho, Young-Hoon Kim, Christoph Wolf, Hyeon-Dong Lee, and Tae-Woo Lee. Improving the stability of metal halide perovskite materials and light-emitting diodes. Advanced Materials, page 1704587, 2018.

[87] Felix Deschler, Michael Price, Sandeep Pathak, Lina E Klintberg, DavidDominik Jarausch, Ruben Higler, Sven HuİLttner, Tomas Leijtens, Samuel D Stranks, and Henry J Snaith. High photoluminescence efficiency and optically pumped lasing in solution-processed mixed halide perovskite semiconductors. The journal of physical chemistry letters, 5(8):1421-1426, 2014.

[88] Luciana C Schmidt, Antonio Pertegas, Soranyel Gonzalez-Carrero, Olga Malinkiewicz, Said Agouram, Guillermo Minguez Espallargas, Henk J Bolink, Raquel E Galian, and Julia Perez-Prieto. Nontemplate synthesis of 
$\mathrm{CH}_{3} \mathrm{NH}_{3} \mathrm{PbBr}_{3}$ perovskite nanoparticles. Journal of the American Chemical Society, 136(3):850-853, 2014.

[89] Feng Zhang, Haizheng Zhong, Cheng Chen, Xian-gang Wu, Xiangmin Hu, Hailong Huang, Junbo Han, Bingsuo Zou, and Yuping Dong. Brightly luminescent and color-tunable colloidal $\mathrm{CH}_{3} \mathrm{NH}_{3} \mathrm{PbX}_{3}(\mathrm{X}=\mathrm{Br}, \mathrm{I}, \mathrm{Cl})$ quantum dots: potential alternatives for display technology. ACS nano, 9(4):4533-4542, 2015.

[90] Yasser Hassan, Yin Song, Ryan D Pensack, Ahmed I Abdelrahman, Yoichi Kobayashi, Mitchell A Winnik, and Gregory D Scholes. Structure-tuned lead halide perovskite nanocrystals. Advanced Materials, 28(3):566-573, 2016.

[91] Bruno Brunetti, Carmen Cavallo, Andrea Ciccioli, Guido Gigli, and Alessandro Latini. On the thermal and thermodynamic (in) stability of methylammonium lead halide perovskites. Scientific reports, 6:31896, 2016.

[92] Vasco Ronchi and Vinicio Barocas. The nature of light: An historical survey. The nature of light: An historical survey., by Ronchi, V.; Barocas, V.. Cambridge, MA (USA): Harvard University Press, 12+ 288 p., 1970.

[93] Seong-Rin Lim, Daniel Kang, Oladele A Ogunseitan, and Julie M Schoenung. Potential environmental impacts from the metals in incandescent, compact fluorescent lamp (CFL), and light-emitting diode (LED) bulbs. Environmental science \& technology, 47(2):1040-1047, 2012.

[94] Simon M Sze and Kwok K Ng. Physics of semiconductor devices. John wiley \& sons, 2006.

[95] Jeffrey A Hart, Stefanie Ann Lenway, and Thomas Murtha. A history of electroluminescent displays. Indiana University, pages 1-18, 1999.

[96] Robert Besancon. The encyclopedia of physics. Springer Science \& Business Media, 2013. 
[97] Rubin Braunstein. Radiative transitions in semiconductors. Physical Review, 99(6):1892, 1955.

[98] Thomas M Okon and James R Biard. The first practical LED. Edison Tech Center, pages 1-14, 2015.

[99] James R Biard and Gary E Pittman. Semiconductor radiant diode, December 20 1966. US Patent 3,293,513.

[100] Nick Holonyak Jr and SF Bevacqua. Coherent (visible) light emission from Ga(As $\left.{ }_{1-x} \mathrm{P}_{x}\right)$ junctions. Applied Physics Letters, 1(4):82-83, 1962.

[101] Tekla S Perry. M. George Craford [biography]. IEEE Spectrum, 32(2):5255, 1995.

[102] Neel V Patel. Nobel shocker: RCA had the first blue LED in 1972. IEEE Spectrum, Online Article, 2014.

[103] SI Vlaskina. Silicon carbide led. Semiconductor Physics Quantum Electronics \& Optoelectronics, 2002.

[104] Shuji Nakamura, Takashi Mukai, and Masayuki Senoh. Candelaclass high-brightness InGaN/AlGaN double-heterostructure blue-lightemitting diodes. Applied Physics Letters, 64(13):1687-1689, 1994.

[105] A Dadgar, A Alam, T Riemann, J Bläsing, A Diez, M Poschenrieder, M Strassburg, M Heuken, J Christen, and A Krost. Crack-free InGaN/GaN light emitters on Si (111). physica status solidi (a), 188(1):155-158, 2001.

[106] A Dadgar, M Poschenrieder, J Bläsing, K Fehse, A Diez, and A Krost. Thick, crack-free blue light-emitting diodes on Si (111) using low-temperature AlN interlayers and in situ Si x N y masking. Applied Physics Letters, 80(20):3670-3672, 2002.

[107] Isamu Akasaki, Hiroshi Amano, and Shuji Nakamura. Blue LEDs-filling the world with new light. Nobel Prize, 2014. 
[108] Nadarajah Narendran, Y Gu, JP Freyssinier, H Yu, and L Deng. Solid-state lighting: failure analysis of white LEDs. Journal of Crystal Growth, 268(34):449-456, 2004.

[109] Isamu Akasaki, Hiroshi Amano, and Shuji Nakamura. The Nobel prize in Physics 2014. The Royal Swedish Academy of Science, 2014.

[110] Setsuhisa Tanabe, Shunsuke Fujita, Satoru Yoshihara, Akihiko Sakamoto, and Shigeru Yamamoto. YAG glass-ceramic phosphor for white LED (II): luminescence characteristics. In Fifth International Conference on Solid State Lighting, volume 5941, page 594112. International Society for Optics and Photonics, 2005.

[111] Mark T Bernius, Mike Inbasekaran, Jim O’Brien, and Weishi Wu. Progress with light-emitting polymers. Advanced Materials, 12(23):1737-1750, 2000 .

[112] Ching W Tang and Steven A VanSlyke. Organic electroluminescent diodes. Applied physics letters, 51(12):913-915, 1987.

[113] Daniel J Gaspar and Evgueni Polikarpov. OLED fundamentals: materials, devices, and processing of organic light-emitting diodes. CRC Press, 2015.

[114] Akiyoshi Mikami, Tatsuya Koshiyama, and Tetsuro Tsubokawa. Highefficiency color and white organic light-emitting devices prepared on flexible plastic substrates. Japanese journal of applied physics, 44(1S):608, 2005.

[115] GE Jabbour, Y Kawabe, SE Shaheen, JF Wang, MM Morrell, B Kippelen, and N Peyghambarian. Highly efficient and bright organic electroluminescent devices with an aluminum cathode. Applied physics letters, 71(13):1762-1764, 1997.

[116] Akiyoshi Mikami, Yusuke Nishita, and Yoichi Iida. 35-3: High efficiency phosphorescent organic light-emitting devices coupled with lat- 
eral color-conversion layer. In SID symposium digest of technical papers, volume 37, pages 1376-1379. Wiley Online Library, 2006.

[117] Hepeng Jia. Who will win the future of display technologies? National Science Review, 2018.

[118] Marius Grundmann. Nano-optoelectronics: concepts, physics and devices. Springer Science \& Business Media, 2002.

[119] Tomoya Sugahara, Hisao Sato, Maosheng Hao, Yoshiki Naoi, Satoshi Kurai, Satoru Tottori, Kenji Yamashita, Katsushi Nishino, Linda T Romano, and Shiro Sakai. Direct evidence that dislocations are non-radiative recombination centers in GaN. Japanese journal of applied physics, 37(4A):L398, 1998.

[120] Hyunki Kim, Ji Yeon Han, Dong Seok Kang, Sung Wook Kim, Dong Seon Jang, Minwon Suh, Artavazd Kirakosyan, and Duk Young Jeon. Characteristics of $\mathrm{CuInS}_{2} / \mathrm{ZnS}$ quantum dots and its application on LED. Journal of Crystal Growth, 326(1):90-93, 2011.

[121] Joong Pill Park, Jae-Joon Lee, and Sang-Wook Kim. Highly luminescent $\mathrm{InP} / \mathrm{GaP} / \mathrm{ZnS}$ QDs emitting in the entire color range via a heating up process. Scientific Reports, 6, 2016.

[122] Xuyong Yang, Dewei Zhao, Kheng Swee Leck, Swee Tiam Tan, Yu Xin Tang, Junliang Zhao, Hilmi Volkan Demir, and Xiao Wei Sun. Full visible range covering $\mathrm{InP} / \mathrm{ZnS}$ nanocrystals with high photometric performance and their application to white quantum dot light-emitting Diodes. Advanced Materials, 24(30):4180-4185, 2012.

[123] Yasuhiro Shirasaki, Geoffrey J Supran, Moungi G Bawendi, and Vladimir Bulović. Emergence of colloidal quantum-dot light-emitting technologies. Nature Photonics, 7(1):13-23, 2013. 
[124] Yuanyuan Su, Yao He, Haoting Lu, Liman Sai, Qingnuan Li, Wenxin Li, Lianhui Wang, Pingping Shen, Qing Huang, and Chunhai Fan. The cytotoxicity of cadmium based, aqueous phase-synthesized, quantum dots and its modulation by surface coating. Biomaterials, 30(1):19-25, 2009.

[125] Maria Luiza FM Kede, Fabio V Correia, Paulo F Conceição, Sidney F Salles Junior, Marcia Marques, Josino C Moreira, and Daniel V Pérez. Evaluation of mobility, bioavailability and toxicity of $\mathrm{Pb}$ and $\mathrm{Cd}$ in contaminated soil using TCLP, BCR and earthworms. International journal of environmental research and public health, 11(11):11528-11540, 2014.

[126] Chenxin Ran, Zhaoxin Wu, Jun Xi, Fang Yuan, Hua Dong, Ting Lei, Xin $\mathrm{He}$, and Xun Hou. Construction of compact methylammonium bismuth iodide film promoting lead-free inverted planar heterojunction organohalide solar cells with open-circuit voltage over $0.8 \mathrm{~V}$. The Journal of Physical Chemistry Letters, 8(2):394-400, 2017.

[127] Adam H Slavney, $\mathrm{Te} \mathrm{Hu}$, Aaron M Lindenberg, and Hemamala I Karunadasa. A bismuth-halide double perovskite with long carrier recombination lifetime for photovoltaic applications. Journal of the American Chemical Society, 138(7):2138-2141, 2016.

[128] Guangru Li, Florencia Wisnivesky Rocca Rivarola, Nathaniel JLK Davis, Sai Bai, Tom C Jellicoe, Francisco de la PeÃśa, Shaocong Hou, Caterina Ducati, Feng Gao, and Richard H Friend. Highly efficient perovskite nanocrystal light-emitting diodes enabled by a universal crosslinking method. Advanced Materials, 28(18):3528-3534, 2016.

[129] Jun Pan, Li Na Quan, Yongbiao Zhao, Wei Peng, Banavoth Murali, Smritakshi P Sarmah, Mingjian Yuan, Lutfan Sinatra, Noktan M Alyami, and Jiakai Liu. Highly efficient perovskite-quantum-dot light-emitting diodes by surface engineering. Advanced Materials, 28(39):8718-8725, 2016. 
[130] Xiaoyu Zhang, Hong Lin, He Huang, Claas Reckmeier, Yu Zhang, Wallace CH Choy, and Andrey L Rogach. Enhancing the brightness of cesium lead halide perovskite nanocrystal based green light-emitting devices through the interface engineering with perfluorinated ionomer. Nano Letters, 16(2):1415-1420, 2016.

[131] En-Ping Yao, Zhanlue Yang, Lei Meng, Pengyu Sun, Shiqi Dong, Ye Yang, and Yang Yang. High-brightness blue and white LEDs based on inorganic perovskite nanocrystals and their composites. Advanced Materials, 29(23), 2017.

[132] Chun Sun, Yu Zhang, Cheng Ruan, Chunyang Yin, Xiaoyong Wang, Yiding Wang, and William W Yu. Efficient and Stable White LEDs with Silica-Coated Inorganic Perovskite Quantum Dots. Advanced Materials, 28(45):10088-0094, 2016.

[133] Xin Yu Chin, Ajay Perumal, Annalisa Bruno, Natalia Yantara, Sjoerd A Veldhuis, Laura Martínez-Sarti, Bevita Chandran, Vladimir Chirvony, Alencious Shu-Zee Lo, Jinkyu So, et al. Self-assembled hierarchical nanostructured perovskites enable highly efficient LEDs via an energy cascade. Energy \& Environmental Science, 2018.

[134] Haider Baqer Salman. Synthesis, characterization, and fabrication of all inorganic quantum dot LEDs. PhD thesis, University of Arkansas, 2016.

[135] Guangru Li, Zhi-Kuang Tan, Dawei Di, May Ling Lai, Lang Jiang, Jonathan Hua-Wei Lim, Richard H Friend, and Neil C Greenham. Efficient lightemitting diodes based on nanocrystalline perovskite in a dielectric polymer matrix. Nano Letters, 15(4):2640-2644, 2015.

[136] Michael Kulbak, David Cahen, and Gary Hodes. How important is the organic part of lead halide perovskite photovoltaic cells? efficient $\mathrm{CsPbBr}_{3}$ Cells. The journal of physical chemistry letters, 6(13):2452-2456, 2015. 
[137] Jinwoo Byun, Himchan Cho, Christoph Wolf, Mi Jang, Aditya Sadhanala, Richard H Friend, Hoichang Yang, and TaeâĂ̌̌Woo Lee. Efficient visible quasi-2D perovskite light-emitting diodes. Advanced Materials, 28(34):7515-7520, 2016.

[138] Venda J Porter, Scott Geyer, Jonathan E Halpert, Marc A Kastner, and Moungi G Bawendi. Photoconduction in annealed and chemically treated CdSe/ZnS inorganic nanocrystal films. The Journal of Physical Chemistry C, 112(7):2308-2316, 2008.

[139] John E Mahan. Physical vapor deposition of thin films. Physical Vapor Deposition of Thin Films, by John E. Mahan, pp. 336. ISBN 0-471-330019. Wiley-VCH, January 2000., page 336, 2000.

[140] Michael S Shur. Handbook series on semiconductor parameters, volume 1. World Scientific, 1996.

[141] Siddha Pimputkar, James S Speck, Steven P DenBaars, and Shuji Nakamura. Prospects for LED lighting. Nature photonics, 3(4):180, 2009.

[142] YC Shen, GO Mueller, S Watanabe, NF Gardner, A Munkholm, and MR Krames. Auger recombination in InGaN measured by photoluminescence. Applied Physics Letters, 91(14):141101, 2007.

[143] Wan Ki Bae, Young-Shin Park, Jaehoon Lim, Donggu Lee, Lazaro A Padilha, Hunter McDaniel, Istvan Robel, Changhee Lee, Jeffrey M Pietryga, and Victor I Klimov. Controlling the influence of Auger recombination on the performance of quantum-dot light-emitting diodes. $\mathrm{Na}$ ture communications, 4:2661, 2013.

[144] Richard Stevenson. The LED's dark secret. IEEE Spectrum, 46(8), 2009.

[145] K. T. a. Y. S A. T. Kojima. Novel photoelectrochemical cell with mesoscopic electrodes sensitized by lead-halide compounds. ECS meeting, page 397, 2006. 
[146] Akihiro Kojima, Kenjiro Teshima, Yasuo Shirai, and Tsutomu Miyasaka. Organometal halide perovskites as visible-light sensitizers for photovoltaic cells. Journal of the American Chemical Society, 131(17):60506051, 2009.

[147] Jeong-Hyeok Im, Chang-Ryul Lee, Jin-Wook Lee, Sang-Won Park, and Nam-Gyu Park. $6.5 \%$ efficient perovskite quantum-dot-sensitized solar cell. Nanoscale, 3(10):4088-4093, 2011.

[148] Hui-Seon Kim, Chang-Ryul Lee, Jeong-Hyeok Im, Ki-Beom Lee, Thomas Moehl, Arianna Marchioro, Soo-Jin Moon, Robin Humphry-Baker, JunHo Yum, and Jacques E Moser. Lead iodide perovskite sensitized allsolid-state submicron thin film mesoscopic solar cell with efficiency exceeding 9 \%. Scientific reports, 2, 2012.

[149] Michael M Lee, JoÃńl Teuscher, Tsutomu Miyasaka, Takurou N Murakami, and Henry J Snaith. Efficient hybrid solar cells based on mesosuperstructured organometal halide perovskites. Science, 338(6107):643$647,2012$.

[150] Mingzhen Liu, Michael B Johnston, and Henry J Snaith. Efficient planar heterojunction perovskite solar cells by vapour deposition. Nature, 501(7467):395-398, 2013.

[151] Nam Joong Jeon, Jun Hong Noh, Young Chan Kim, Woon Seok Yang, Seungchan Ryu, and Sang Il Seok. Solvent engineering for highperformance inorganic-organic hybrid perovskite solar cells. Nature materials, 13(9):897, 2014.

[152] Jun Hong Noh, Sang Hyuk Im, Jin Hyuck Heo, Tarak N Mandal, and Sang Il Seok. Chemical management for colorful, efficient, and stable inorganicorganic hybrid nanostructured solar cells. Nano Letters, 13(4):1764-1769, 2013. 
[153] Woon Seok Yang, Byung-Wook Park, Eui Hyuk Jung, Nam Joong Jeon, Young Chan Kim, Dong Uk Lee, Seong Sik Shin, Jangwon Seo, Eun Kyu Kim, Jun Hong Noh, et al. Iodide management in formamidiniumlead-halide-based perovskite layers for efficient solar cells. Science, 356(6345):1376-1379, 2017.

[154] Victor Drits, Jan Srodon, and DD Eberl. XRD measurement of mean crystallite thickness of illite and illite/smectite: Reappraisal of the Kubler index and the Scherrer equation. Clays and clay minerals, 45(3):461-475, 1997.

[155] Andrew R. Barron. Physical methods in Chemistry and nano Science. OpenStax-CNX, 2014.

[156] Jerry Workman Jr and Art Springsteen. Applied spectroscopy: a compact reference for practitioners. Academic Press, 1998.

[157] Holger Borchert. Absorption and photoluminescence spectroscopy. In Solar Cells Based on Colloidal Nanocrystals, pages 119-127. Springer, 2014.

[158] Liuqi Zhang, Xiaolei Yang, Qi Jiang, Pengyang Wang, Zhigang Yin, Xingwang Zhang, Hairen Tan, Yang Michael Yang, Mingyang Wei, Brandon R Sutherland, et al. Ultra-bright and highly efficient inorganic based perovskite light-emitting diodes. Nature communications, 8:15640, 2017.

[159] Walter Stanley Stiles, BH Crawford, et al. The luminous efficiency of rays entering the eye pupil at different points. Proc. R. Soc. Lond. B, 112(778):428-450, 1933.

[160] C CIE. Commission internationale de l'eclairage proceedings, 1931. Cambridge University Press Cambridge, 1932.

[161] Thomas Smith and John Guild. The CIE colorimetric standards and their use. Transactions of the optical society, 33(3):73, 1931. 
[162] Chia-Hao M Chuang, Patrick R Brown, Vladimir Bulović, and Moungi G Bawendi. Improved performance and stability in quantum dot solar cells through band alignment engineering. Nature materials, 13(8):796, 2014.

[163] Zheng Li and Xiaogang Peng. Size/shape-controlled synthesis of colloidal CdSe quantum disks: ligand and temperature effects. Journal of the American Chemical Society, 133(17):6578-6586, 2011.

[164] Anne C Berends and Celso de Mello Donega. Ultrathin one-and twodimensional colloidal semiconductor nanocrystals: pushing quantum confinement to the limit. The Journal of Physical Chemistry Letters, 8(17):4077-4090, 2017.

[165] Samuel D Stranks, Giles E Eperon, Giulia Grancini, Christopher Menelaou, Marcelo JP Alcocer, Tomas Leijtens, Laura M Herz, Annamaria Petrozza, and Henry J Snaith. Electron-hole diffusion lengths exceeding 1 micrometer in an organometal trihalide perovskite absorber. Science, 342(6156):341-344, 2013.

[166] Arun Singh Chouhan, Naga Prathibha Jasti, Shreyash Hadke, Srinivasan Raghavan, and Sushobhan Avasthi. Large grained and high charge carrier lifetime $\mathrm{CH}_{3} \mathrm{NH}_{3} \mathrm{PbI}_{3}$ thin-films: implications for perovskite solar cells. Current Applied Physics, 17(10):1335-1340, 2017.

[167] Feng Wang, Sai Bai, Wolfgang Tress, Anders Hagfeldt, and Feng Gao. Defects engineering for high-performance perovskite solar cells. npj Flexible Electronics, 2(1):22, 2018.

[168] Weiqiang Chen, Saikat Bhaumik, Sjoerd A Veldhuis, Guichuan Xing, Qiang Xu, Michael GrÃd'tzel, Subodh Mhaisalkar, Nripan Mathews, and Tze Chien Sum. Giant five-photon absorption from multidimensional core-shell halide perovskite colloidal nanocrystals. Nature communications, 8:15198, 2017. 
[169] Guangru Li, Michael Price, and Felix Deschler. Research Update: Challenges for high-efficiency hybrid lead-halide perovskite LEDs and the path towards electrically pumped lasing. APL Materials, 4(9):091507, 2016.

[170] Erol Kucur, Jürgen Riegler, Gerald A Urban, and Thomas Nann. Determination of quantum confinement in CdSe nanocrystals by cyclic voltammetry. The Journal of chemical physics, 119(4):2333-2337, 2003.

[171] A Paul Alivisatos. Semiconductor clusters, nanocrystals, and quantum dots. science, 271(5251):933-937, 1996.

[172] William L Wilson, PF Szajowski, and LE Brus. Quantum confinement in size-selected, surface-oxidized silicon nanocrystals. Science, 262(5137):1242-1244, 1993.

[173] Lianhua Qu and Xiaogang Peng. Control of photoluminescence properties of CdSe nanocrystals in growth. Journal of the American Chemical Society, 124(9):2049-2055, 2002.

[174] Manoj Nirmal, David J Norris, Masaru Kuno, Moungi G Bawendi, Al L Efros, and M Rosen. Observation of the "dark exciton" in CdSe quantum dots. Physical review letters, 75(20):3728, 1995.

[175] Y Sonnefraud, N Chevalier, J-F Motte, S Huant, P Reiss, J Bleuse, F Chandezon, MT Burnett, W Ding, and SA Maier. Near-field optical imaging with a CdSe single nanocrystal-based active tip. Optics express, 14(22):10596-10602, 2006.

[176] Y Chan, J-Michel Caruge, PT Snee, and MG Bawendi. Multiexcitonic two-state lasing in a CdSe nanocrystal laser. Applied physics letters, 85(13):2460-462, 2004.

[177] Yinthai Chan, Jonathan S Steckel, Preston T Snee, J-Michel Caruge, Justin M Hodgkiss, Daniel G Nocera, and Moungi G Bawendi. Blue semiconductor nanocrystal laser. Applied Physics Letters, 86(7):073102, 2005. 
[178] Rebecca Somers, Moungi Bawendi, and Daniel G Nocera. CdSe nanocrystal based chem-/bio-sensors. Chemical Society Reviews, 36(4):579-591, 2007.

[179] Yunfei Zhou, Frank S Riehle, Ying Yuan, Hans-Frieder Schleiermacher, Michael Niggemann, Gerald A Urban, and Michael Krüger. Improved efficiency of hybrid solar cells based on non-ligand-exchanged CdSe quantum dots and poly (3-hexylthiophene). Applied Physics Letters, 96(1):4, 2010 .

[180] Christian Kirchner, Tim Liedl, Stefan Kudera, Teresa Pellegrino, Almudena Muñoz Javier, Hermann E Gaub, Sonja Stölzle, N Fertig, and Wolfgang J Parak. Cytotoxicity of colloidal CdSe and CdSe/ZnS nanoparticles. Nano letters, 5(2):331-338, 2005.

[181] Younghoon Kim, Emre Yassitepe, Oleksandr Voznyy, Riccardo Comin, Grant Walters, Xiwen Gong, Pongsakorn Kanjanaboos, Ana F Nogueira, and Edward H Sargent. Efficient luminescence from perovskite quantum dot solids. ACS applied materials \& interfaces, 7(45):25007-25013, 2015.

[182] I Ya Kuznetsova, IS Kovaleva, and VA Fedorov. Interaction of lead bromide with cesium and cadmium bromides. Zhurnal Neorganicheskoj Khimii, 46(11):1900-1905, 2001.

[183] A Polimeni, A Patane, M Grassi Alessi, M Capizzi, F Martelli, A Bosacchi, and S Franchi. . Physical Review B, 54(23):16389, 1996.

[184] Frank W Wise. Lead salt quantum dots: the limit of strong quantum confinement. Accounts of Chemical Research, 33(11):773-780, 2000.

[185] Louis E Brus. Electron-electron and electron-hole interactions in small semiconductor crystallites: The size dependence of the lowest excited electronic state. The Journal of chemical physics, 80(9):4403-4409, 1984. 
[186] Louis Brus. Electronic wave functions in semiconductor clusters: experiment and theory. The Journal of Physical Chemistry, 90(12):2555-2560, 1986.

[187] Joseph S Manser and Prashant V Kamat. Band filling with free charge carriers in organometal halide perovskites. Nature Photonics, 8(9):737, 2014.

[188] Justinas Butkus, Parth Vashishtha, Kai Chen, Joseph K Gallaher, Shyamal KK Prasad, Dani Z Metin, Geoffry Laufersky, Nicola Gaston, Jonathan E Halpert, and Justin M Hodgkiss. The evolution of quantum confinement in $\mathrm{CsPbBr}_{3}$ perovskite nanocrystals. Chemistry of Materials, 2017.

[189] Surendra Sharma, Norbert Weiden, and Alarich Weiss. Phase diagrams of quasibinary systems of the type: $\mathrm{ABX}_{3}-\mathrm{A}^{\prime} \mathrm{BX}_{3} ; \mathrm{ABX}_{3}-\mathrm{AB}^{\prime} \mathrm{X}_{3}$, and $\mathrm{ABX}_{3}-$ $\mathrm{ABX}_{3} ; \mathrm{X}=$ halogen. Zeitschrift für Physikalische Chemie, 175(1):63-80, 1992.

[190] Ralph G Pearson. Hard and soft acids and bases. Journal of the American Chemical Society, 85(22):3533-3539, 1963.

[191] Dandan Zhang, Yi Yu, Yehonadav Bekenstein, Andrew B Wong, A Paul Alivisatos, and Peidong Yang. Ultrathin colloidal cesium lead halide perovskite nanowires. Journal of the American Chemical Society, 138(40):13155-13158, 2016.

[192] Zhenyu Yang, James Z Fan, Andrew H Proppe, F Pelayo García de Arquer, David Rossouw, Oleksandr Voznyy, Xinzheng Lan, Min Liu, Grant Walters, Rafael Quintero-Bermudez, et al. Mixed-quantum-dot solar cells. Nature communications, 8(1):1325, 2017.

[193] Samad Mussa Farkhani and Alireza Valizadeh. three synthesis methods of $\mathrm{CdX}(\mathrm{X}=\mathrm{Se}, \mathrm{S}$ or Te) quantum dots. IET nanobiotechnology, 8(2):59-76, 2014. 
[194] Jaakko VI Timonen, Eira T Seppälä, Olli Ikkala, and Robin HA Ras. From hot-injection synthesis to heating-up synthesis of cobalt nanoparticles: Observation of kinetically controllable nucleation. Angewandte Chemie International Edition, 50(9):2080-2084, 2011.

[195] Polina O Anikeeva, Jonathan E Halpert, Moungi G Bawendi, and Vladimir Bulovic. Electroluminescence from a mixed red-green-blue colloidal quantum dot monolayer. Nano Letters, 7(8):2196-2200, 2007.

[196] Muhazri Abd Mutalib, Norasikin Ahmad Ludin, Nik Ahmad Aizudden Nik Ruzalman, Vincent Barrioz, Suhaila Sepeai, Mohd Asri Mat Teridi, Mohd Sukor SuâĂŹait, Mohd Adib Ibrahim, and Kamaruzzaman Sopian. Progress towards highly stable and lead-free perovskite solar cells. Materials for Renewable and Sustainable Energy, 7(2):7, 2018.

[197] Yimin Gu, Nadarajah Narendran, and Jean Paul Freyssinier. White LED performance. In Fourth international conference on solid state lighting, volume 5530, pages 119-125. International Society for Optics and Photonics, 2004.

[198] Long Hu, Gang Shao, Tao Jiang, Dengbing Li, Xinlin Lv, Hongya Wang, Xinsheng Liu, Haisheng Song, Jiang Tang, and Huan Liu. Investigation of the interaction between perovskite films with moisture via in situ electrical resistance measurement. ACS applied materials \& interfaces, 7(45):25113-25120, 2015.

[199] Gabriel Bernardo, Ana Charas, and Jorge Morgado. Luminescence properties of poly (9, 9-dioctylfluorene)/polyvinylcarbazole blends: Role of composition on the emission colour stability and electroluminescence efficiency. Journal of Physics and Chemistry of Solids, 71(3):340-345, 2010 .

[200] Wan Ki Bae, Jaehoon Lim, Matthias Zorn, Jeonghun Kwak, Young-Shin Park, Donggu Lee, Seonghoon Lee, Kookheon Char, Rudolf Zentel, and 
Changhee Lee. Reduced efficiency roll-off in light-emitting diodes enabled by quantum dot-conducting polymer nanohybrids. Journal of Materials Chemistry C, 2(25):4974-4979, 2014.

[201] Quyet Van Le, Minjoon Park, Woonbae Sohn, Ho Won Jang, and Soo Young Kim. Investigation of energy levels and crystal structures of cesium lead halides and their application in full-color light-emitting diodes. Advanced Electronic Materials, 3(1), 2017.

[202] A Pietraszewska-Bogiel and TWJ Gadella. FRET microscopy: from principle to routine technology in cell biology. Journal of microscopy, 241(2):111-118, 2011.

[203] Hilmi Volkan Demir, Sedat Nizamoglu, Talha Erdem, Evren Mutlugun, Nikolai Gaponik, and Alexander Eychmüller. Quantum dot integrated LEDs using photonic and excitonic color conversion. Nano Today, 6(6):632-647, 2011.

[204] Stephen A Empedocles and Moungi G Bawendi. Quantum-confined stark effect in single CdSe nanocrystallite quantum dots. Science, 278(5346):2114-2117, 1997.

[205] DONG ICK SON, JAEHO SHIM, and KYU SEUNG LEE. Nanophotonics: Optoelectronic devices based on hybrid quantum dots and carbon nanomaterials. AAPPS Bulletin, 25(5), 2015.

[206] Ching Wan Tang, Steven A VanSlyke, and CH Chen. Electroluminescence of doped organic thin films. Journal of Applied Physics, 65(9):3610-3616, 1989.

[207] Liang Li, Nelson Coates, and Daniel Moses. Solution-processed inorganic solar cell based on in situ synthesis and film deposition of $\mathrm{CuInS}_{2}$ nanocrystals. Journal of the American Chemical Society, 132(1):22-23, 2009. 
[208] Leyre Gomez, Junhao Lin, Chris De Weerd, Lucas Poirier, Simon C Boehme, Elizabeth Von Hauff, Yasufumi Fujiwara, Kazutomo Suenaga, and Tom Gregorkiewicz. Extraordinary interfacial stitching between single all-inorganic perovskite nanocrystals. ACS applied materials \& interfaces, 10(6):5984-5991, 2018.

[209] CBea Murray, David J Norris, and Moungi G Bawendi. Synthesis and characterization of nearly monodisperse $\mathrm{CdE}$ (E- sulfur, selenium, tellurium) semiconductor nanocrystallites. Journal of the American Chemical Society, 115(19):8706-8715, 1993.

[210] Jagdeep Shah, A Pinczuk, HL Störmer, AC Gossard, and W Wiegmann. Electric field induced heating of high mobility electrons in modulationdoped GaAs-AlGaAs heterostructures. Applied Physics Letters, 42(1):5557, 1983.

[211] Bohee Hwang, Chungwan Gu, Donghwa Lee, and Jang-Sik Lee. Effect of halide-mixing on the switching behaviors of organic-inorganic hybrid perovskite memory. Scientific Reports, 7:43794, 2017.

[212] Sergii Yakunin, Loredana Protesescu, Franziska Krieg, Maryna I Bodnarchuk, Georgian Nedelcu, Markus Humer, Gabriele De Luca, Manfred Fiebig, Wolfgang Heiss, and Maksym V Kovalenko. Low-threshold amplified spontaneous emission and lasing from colloidal nanocrystals of caesium lead halide perovskites. Nature communications, 6, 2015.

[213] C Javaux, B Mahler, B Dubertret, Andrew Shabaev, AV Rodina, Al L Efros, DR Yakovlev, F Liu, M Bayer, and G Camps. Thermal activation of nonradiative Auger recombination in charged colloidal nanocrystals. Nature nanotechnology, 8(3):206-212, 2013.

[214] Georgian Nedelcu, Loredana Protesescu, Sergii Yakunin, Maryna I Bodnarchuk, Matthias J Grotevent, and Maksym V Kovalenko. Fast anion- 
exchange in highly luminescent nanocrystals of cesium lead halide perovskites (CsPbX 3 , X= Cl, Br, I). Nano Letters, 15(8):5635-5640, 2015.

[215] Rebecca J Sutton, Giles E Eperon, Laura Miranda, Elizabeth S Parrott, Brett A Kamino, Jay B Patel, Maximilian T Horantner, Michael B Johnston, Amir Abbas Haghighirad, and David T Moore. Bandgap-tunable cesium lead halide perovskites with high thermal stability for efficient solar cells. Advanced Energy Materials, 2016.

[216] Matthew J Panzer, Polina O Anikeeva, Jonathan E Halpert, Moungi G Bawendi, and Vladimir Bulovic. Nanoscale investigation of collodial quantum dot/organic semiconductor interfaces. In Optics and Photonics for Advanced Energy Technology, page WC5. Optical Society of America.

[217] Jianhai Li, Leimeng Xu, Tao Wang, Jizhong Song, Jiawei Chen, Jie Xue, Yuhui Dong, Bo Cai, Qingsong Shan, Boning Han, et al. 50-old EQE improvement up to $6.27 \%$ of solution-processed all-inorganic perovskite $\mathrm{CsPbBr}_{3}$ QLEDs via surface ligand density control. Advanced Materials, 29(5), 2017.

[218] Xiaoli Zhang, Bing Xu, Jinbao Zhang, Yuan Gao, Yuanjin Zheng, Kai Wang, and Xiao Wei Sun. All-inorganic perovskite nanocrystals for highefficiency light emitting diodes: Dual-phase $\mathrm{CsPbBr}_{3}-\mathrm{CsPb}_{2} \mathrm{Br}_{5}$ composites. Advanced Functional Materials, 26(25):4595-4600, 2016.

[219] X Hong, T Ishihara, and AV Nurmikko. Dielectric confinement effect on excitons in $\mathrm{PbI}_{4}$-based layered semiconductors. Physical Review B, 45(12):6961, 1992.

[220] T Umebayashi, K Asai, T Kondo, and A Nakao. Electronic structures of lead iodide based low-dimensional crystals. Physical Review B, 67(15):155405, 2003.

[221] Hongwei Hu, Teddy Salim, Bingbing Chen, and Yeng Ming Lam. Molecularly engineered organic-inorganic hybrid perovskite with multiple 
quantum well structure for multicolored light-emitting diodes. Scientific reports, 6:33546, 2016.

[222] Ian C Smith, Eric T Hoke, Diego Solis-Ibarra, Michael D McGehee, and Hemamala I Karunadasa. A layered hybrid perovskite solar-cell absorber with enhanced moisture stability. Angewandte Chemie, 126(42):1141411417, 2014.

[223] Duyen H Cao, Constantinos C Stoumpos, Omar K Farha, Joseph T Hupp, and Mercouri G Kanatzidis. 2D homologous perovskites as lightabsorbing materials for solar cell applications. Journal of the American Chemical Society, 137(24):7843-7850, 2015.

[224] Jin Chang, Shuting Zhang, Nana Wang, Yan Sun, Yingqiang Wei, Renzhi Li, Chang Yi, Jianpu Wang, and Wei Huang. Enhanced performance of red perovskite light-emitting diodes through the dimensional tailoring of perovskite multiple quantum wells. The journal of physical chemistry letters, 9(4):881-886, 2018.

[225] Young-Hoon Kim, Himchan Cho, Jin Hyuck Heo, Tae-Sik Kim, NoSoung Myoung, Chang-Lyoul Lee, Sang Hyuk Im, and Tae-Woo Lee. Multicolored organic/inorganic hybrid perovskite light-emitting diodes. Advanced materials, 27(7):1248-1254, 2015.

[226] C Janáky and K Rajeshwar. The role of (photo) electrochemistry in the rational design of hybrid conducting polymer/semiconductor assemblies: From fundamental concepts to practical applications. Progress in Polymer Science, 43:96-135, 2015.

[227] Giles E Eperon, Giuseppe M PaternÃš, Rebecca J Sutton, Andrea Zampetti, Amir Abbas Haghighirad, Franco Cacialli, and Henry J Snaith. Inorganic caesium lead iodide perovskite solar cells. Journal of Materials Chemistry A, 3(39):19688-19695, 2015. 
[228] Ming Yang, Nana Wang, Shuting Zhang, Wei Zou, Yarong He, Yingqiang Wei, Mengmeng $\mathrm{Xu}$, Jianpu Wang, and Wei Huang. Reduced efficiency roll-off and enhanced stability in perovskite light-emitting diodes with multiple quantum wells. The journal of physical chemistry letters, 2018.

[229] Qi Wang, Jie Ren, Xue-Feng Peng, Xia-Xia Ji, and Xiao-Hui Yang. Efficient sky-blue perovskite light-emitting devices based on ethylammonium bromide induced layered perovskites. ACS applied materials \& interfaces, 9(35):29901-29906, 2017.

[230] Sheng-Wen Wang, Huang-Yu Lin, Chien-Chung Lin, Tsung Sheng Kao, Kuo-Ju Chen, Hau-Vei Han, Jie-Ru Li, Po-Tsung Lee, Huang-Ming Chen, Ming-Hui Hong, et al. Pulsed-laser micropatterned quantum-dot array for white light source. Scientific reports, 6:23563, 2016.

[231] Pengfei Fu, Qingsong Shan, Yuequn Shang, Jizhong Song, Haibo Zeng, Zhijun Ning, and Jinkang Gong. Perovskite nanocrystals: synthesis, properties and applications. Science Bulletin, 2017.

[232] Hailong Huang, Fangchao Zhao, Lige Liu, Feng Zhang, Xian-gang Wu, Lijie Shi, Bingsuo Zou, Qibing Pei, and Haizheng Zhong. Emulsion synthesis of size-tunable $\mathrm{CH}_{3} \mathrm{NH}_{3} \mathrm{PbBr}_{3}$ quantum dots: An alternative route toward efficient light-emitting diodes. ACS applied materials \& interfaces, 7(51):28128-28133, 2015.

[233] Tao Yang, Yapeng Zheng, Zhentao Du, Wenna Liu, Zuobao Yang, Fengmei Gao, Lin Wang, Kuo-Chih Chou, Xinmei Hou, and Weiyou Yang. Superior photodetectors based on all-inorganic perovskite $\mathrm{CsPbI}_{3}$ nanorods with ultrafast response and high stability. ACS nano, 2018.

[234] Quinten A Akkerman, Marina Gandini, Francesco Di Stasio, Prachi Rastogi, Francisco Palazon, Giovanni Bertoni, James M Ball, Mirko Prato, Annamaria Petrozza, and Liberato Manna. Strongly emissive perovskite 
nanocrystal inks for high-voltage solar cells. Nature Energy, 2(2):16194, 2017.

[235] Soranyel Gonzalez-Carrero, Raquel E Galian, and Julia PÃl'rez-Prieto. Maximizing the emissive properties of $\mathrm{CH}_{3} \mathrm{NH}_{3} \mathrm{PbBr}_{3}$ perovskite nanoparticles. Journal of Materials Chemistry A, 3(17):9187-9193, 2015.

[236] Yani Chen, Jiajun Peng, Diqing Su, Xiaoqing Chen, and Ziqi Liang. Efficient and balanced charge transport revealed in planar perovskite solar cells. ACS applied materials \& interfaces, 7(8):4471-4475, 2015.

[237] ByungâĂ̌̌Wook Park, Bertrand Philippe, Xiaoliang Zhang, HÃěkan Rensmo, Gerrit Boschloo, and Erik MJ Johansson. Bismuth based hybrid perovskites $\mathrm{A}_{3} \mathrm{Bi}_{2} \mathrm{I}_{9}$ (A: methylammonium or cesium) for solar cell application. Advanced Materials, 27(43):6806-6813, 2015.

[238] Jian Zhang, Ying Yang, Hui Deng, Umar Farooq, Xiaokun Yang, jahangeer khan, Jiang Tang, and Haisheng Song. High quantum yield blue emission from lead-free inorganic antimony halide perovskite colloidal quantum dots. ACS Nano, 2017.

[239] George Volonakis, Amir Abbas Haghighirad, Rebecca L Milot, Weng H Sio, Marina R Filip, Bernard Wenger, Michael B Johnston, Laura M Herz, Henry J Snaith, and Feliciano Giustino. $\mathrm{Cs}_{2} \mathrm{InAgCl}_{6}$ : A new lead-free halide double perovskite with direct band gap. The journal of physical chemistry letters, 8(4):772-778, 2017.

[240] Wasim J Mir, Metikoti Jagadeeswararao, Shyamashis Das, and Angshuman Nag. Colloidal Mn-doped cesium lead halide perovskite nanoplatelets. ACS Energy Letters, 2(3):537-543, 2017.

[241] Wenyong Liu, Qianglu Lin, Hongbo Li, Kaifeng Wu, IstvÃąn Robel, Jeffrey M Pietryga, and Victor I Klimov. $\mathrm{Mn}^{2+}$ - Doped lead halide perovskite nanocrystals with dual-color emission controlled by halide content. J. Am. Chem. Soc, 138(45):14954-14961, 2016. 
[242] Zhen Li, Mengjin Yang, Ji-Sang Park, Su-Huai Wei, Joseph J Berry, and Kai Zhu. Stabilizing perovskite structures by tuning tolerance factor: formation of formamidinium and cesium lead iodide solid-state alloys. Chemistry of Materials, 28(1):284-292, 2015.

[243] James M Ball, Michael M Lee, Andrew Hey, and Henry J Snaith. Lowtemperature processed meso-superstructured to thin-film perovskite solar cells. Energy \& Environmental Science, 6(6):1739-1743, 2013.

[244] Julian Burschka, Norman Pellet, Soo-Jin Moon, Robin Humphry-Baker, Peng Gao, Mohammad K Nazeeruddin, and Michael Grätzel. Sequential deposition as a route to high-performance perovskite-sensitized solar cells. Nature, 499(7458):316, 2013.

[245] Huanping Zhou, Qi Chen, Gang Li, Song Luo, Tze-bing Song, HsinSheng Duan, Ziruo Hong, Jingbi You, Yongsheng Liu, and Yang Yang. Interface engineering of highly efficient perovskite solar cells. Science, 345(6196):542-546, 2014.

[246] Yingzhuang Ma, Parth Vashishtha, Sunil B Shivarudraiah, Kai Chen, Ye Liu, Justin M Hodgkiss, and Jonathan E Halpert. A hybrid perovskite solar cell modified with copper indium sulfide nanocrystals to enhance hole transport and moisture stability. Solar RRL, 1(8), 2017.

[247] Young-Hoon Kim, Christoph Wolf, Young-Tae Kim, Himchan Cho, Woosung Kwon, Sungan Do, Aditya Sadhanala, Chan Gyung Park, ShiWoo Rhee, Sang Hyuk Im, et al. Highly efficient light-emitting diodes of colloidal metal-halide perovskite nanocrystals beyond quantum size. ACS nano, 11(7):6586-6593, 2017.

[248] Sabine Körbel, Miguel AL Marques, and Silvana Botti. Stability and electronic properties of new inorganic perovskites from high-throughput ab initio calculations. Journal of Materials Chemistry C, 4(15):3157-3167, 2016. 
[249] William M Haynes. CRC handbook of chemistry and physics. CRC press, 2014.

[250] A Leonard and GB Gerber. Mutagenicity, carcinogenicity and teratogenicity of thallium compounds. Mutation Research/Reviews in Mutation Research, 387(1):47-53, 1997.

[251] MG Brik, IV Kityk, NM Denysyuk, OY Khyzhun, SI Levkovets, OV Parasyuk, AO Fedorchuk, and GL Myronchuk. Specific features of the electronic structure of a novel ternary $\mathrm{Tl}_{3} \mathrm{PbI}_{5}$ optoelectronic material. Physical Chemistry Chemical Physics, 16(25):12838-12847, 2014.

[252] Alban Ferrier, Matias Velázquez, J-L Doualan, and Richard Moncorgé. $\mathrm{Pr}^{3+}$-doped $\mathrm{Tl}_{3} \mathrm{PbBr}_{5}$ : a non-hygroscopic, non-linear and low-energy phonon single crystal for the mid-infrared laser application. Applied Physics B: Lasers and Optics, 95(2):287-291, 2009.

[253] Alban Ferrier, Matias Velázquez, Xavier Portier, Jean-Louis Doualan, and Richard Moncorgé. $\mathrm{Tl}_{3} \mathrm{PbBr}_{5}$ : A possible crystal candidate for middle infrared nonlinear optics. Journal of crystal growth, 289(1):357-365, 2006.

[254] M Kocsis. Proposal for a new room temperature X-ray detector-thallium lead iodide. IEEE Transactions on Nuclear Science, 47(6):1945-1947, 2000.

[255] Keitaro Hitomi, Toshiyuki Onodera, Tadayoshi Shoji, and Yukio Hiratate. Thallium lead iodide radiation detectors. In Nuclear Science Symposium Conference Record, 2002 IEEE, volume 1, pages 485-488. IEEE.

[256] Alban Ferrier, Matias Velazquez, and Richard Moncorge. Spectroscopic characterization of $\mathrm{Er}^{3+}$-doped $\mathrm{Tl}_{3} \mathrm{PbBr}_{5}$ for midinfrared laser applications. Physical Review B, 77(7):075122, 2008.

[257] Zhao Liu, Ting Zhang, Yafei Wang, Chenyun Wang, Peng Zhang, Hojjatollah Sarvari, Zhi Chen, and Shibin Li. Electronic properties of a new allinorganic perovskite $\mathrm{TlPbI}_{3}$ simulated by the first principles. Nanoscale research letters, 12(1):232, 2017. 
[258] OY Khyzhun, VL Bekenev, OV Parasyuk, SP Danylchuk, NM Denysyuk, AO Fedorchuk, N AlZayed, and IV Kityk. Single crystal growth and the electronic structure of orthorhombic $\mathrm{Tl}_{3} \mathrm{PbBr}_{5}$ : A novel material for nonlinear optics. Optical Materials, 35(5):1081-1089, 2013.

[259] NS AlZayed, J Ebothé, J Michel, IV Kityk, AO Fedorchuk, OV Parasyuk, and G Myronchuk. Optically stimulated IR non-linear optical effects in the $\mathrm{Tl}_{3} \mathrm{PbCl}_{5}$ nanocrystallites. Physica E: Low-dimensional Systems and Nanostructures, 65:130-134, 2015.

[260] W Stoeger. The crystal structures of $\mathrm{TlPbI}_{3}$ and $\mathrm{Tl}_{4} \mathrm{PbI}_{6}$. Zeitschrift für Naturforschung B, 32(9):975-981, 1977.

[261] OY Khyzhun, VL Bekenev, NM Denysyuk, OV Parasyuk, and AO Fedorchuk. First-principles band-structure calculations and X-ray photoelectron spectroscopy studies of the electronic structure of $\mathrm{TlPb}_{2} \mathrm{Cl}_{5}$. Journal of Alloys and Compounds, 582:802-809, 2014.

[262] OY Khyzhun, VL Bekenev, NM Denysyuk, IV Kityk, P Rakus, AO Fedorchuk, SP Danylchuk, and OV Parasyuk. Single crystal growth and the electronic structure of $\mathrm{TlPb}_{2} \mathrm{Br}_{5}$. Optical Materials, 36(2):251-258, 2013.

[263] HP Beck, M Schramm, and R Haberkorn. The InSnCl ${ }_{3}$-type arrangement: II. high pressure synthesis of $\mathrm{TlPbCl}_{3}$ and of solid solutions containing $\mathrm{Rb}$ or Br. Journal of Solid State Chemistry, 146(2):351-354, 1999.

[264] Mats Johnsson and Peter Lemmens. Crystallography and chemistry of perovskites. arXiv preprint cond-mat/0506606, 2005.

[265] J-S Zhou and JB Goodenough. Universal octahedral-site distortion in orthorhombic perovskite oxides. Physical review letters, 94(6):065501, 2005.

[266] OY Khyzhun, PM Fochuk, IV Kityk, M Piasecki, SI Levkovets, AO Fedorchuk, and OV Parasyuk. Single crystal growth and electronic structure of $\mathrm{TlPbI}_{3}$. Materials Chemistry and Physics, 172:165-172, 2016. 
[267] HâĂ̌̌L Keller. The crystal structure of $\mathrm{Tl}_{3} \mathrm{PbCl}_{5}$. Zeitschrift für anorganische und allgemeine Chemie, 432(1):141-146, 1977.

[268] HâĂ ̌̌L Keller. synthesis and crystal structure of low- $\mathrm{Tl}_{3} \mathrm{PbBr}_{5}$. Zeitschrift für anorganische und allgemeine Chemie, 482(11):154-162, 1981.

[269] NM Denysyuk, VL Bekenev, MV Karpets, OV Parasyuk, SP Danylchuk, and OY Khyzhun. Electronic structure of the high-temperature tetragonal Tl3PbBr5 phase. Journal of Alloys and Compounds, 576:271-278, 2013.

[270] VL Bekenev, O Yu Khyzhun, AK Sinelnichenko, VV Atuchin, OV Parasyuk, OM Yurchenko, Yu Bezsmolnyy, AV Kityk, J Szkutnik, and S calus. Crystal growth and the electronic structure of $\mathrm{Tl}_{3} \mathrm{PbCl}_{5}$. Journal of Physics and Chemistry of Solids, 72(6):705-713, 2011.

[271] Young-wook Jun, Ja-Eung Koo, and Jinwoo Cheon. One-step synthesis of size tuned zinc selenide quantum dots via a temperature controlled molecular precursor approach. Chemical Communications, (14):12431244, 2000.

[272] Georg Kresse and JÃijrgen Furthmüller. Efficiency of ab-initio total energy calculations for metals and semiconductors using a plane-wave basis set. Computational materials science, 6(1):15-50, 1996.

[273] Georg Kresse and JÃijrgen Furthmüller. Efficient iterative schemes for ab initio total-energy calculations using a plane-wave basis set. Physical review B, 54(16):11169, 1996.

[274] Georg Kresse and Jãijrgen Hafner. Ab initio molecular dynamics for liquid metals. Physical Review B, 47(1):558, 1993.

[275] Ruijin Yu, Shengliang Zhong, Na Xue, Hongjuan Li, and Hailong Ma. Synthesis, structure, and peculiar green emission of $\mathrm{NaBaBO}_{3}: \mathrm{Ce}^{3+}$ phosphors. Dalton Transactions, 43(28):10969-10976, 2014. 
[276] Yatin J Mange, Melissa R Dewi, Thomas J Macdonald, William M Skinner, and Thomas Nann. Rapid microwave assisted synthesis of nearly monodisperse aqueous $\mathrm{CuInS}_{2} / \mathrm{ZnS}$ nanocrystals. CrystEngComm, 17(41):7820-7823, 2015.

[277] Yuhai Zhang, Jun Yin, Manas R Parida, Ghada H Ahmed, Jun Pan, Osman M Bakr, Jean-Luc BreİĄdas, and Omar F Mohammed. Directindirect nature of the bandgap in lead-free perovskite nanocrystals. The Journal of Physical Chemistry Letters, 8(14):3173-3177, 2017.

[278] Dong Yu, Congjun Wang, Brian L Wehrenberg, and Philippe GuyotSionnest. Variable range hopping conduction in semiconductor nanocrystal solids. Physical review letters, 92(21):216802, 2004.

[279] Mitsuru Inada, Hiroshi Yamamoto, Manabu Gibo, Rieko Ueda, Ikurou Umezu, Shukichi Tanaka, Tadashi Saitoh, and Akira Sugimura. Crossover from Efros-Shklovskii variable range hopping to nearest-neighbor hopping in silicon nanocrystal random network. Applied Physics Express, 8(10):105001, 2015.

[280] Heng Liu, Alexandre Pourret, and Philippe Guyot-Sionnest. Mott and Efros-Shklovskii variable range hopping in CdSe quantum dots films. Acs Nano, 4(9):5211-5216, 2010.

[281] Jin-Wook Lee, Deok-Hwan Kim, Hui-Seon Kim, Seung-Woo Seo, Sung Min Cho, and Nam-Gyu Park. Formamidinium and cesium hybridization for photo-and moisture-stable perovskite solar cell. Advanced Energy Materials, 5(20), 2015.

[282] Soumya Kundu and Timothy L Kelly. Improving the moisture stability of perovskite solar cells by using PMMA/P3HT based hole-transport layers. Materials Chemistry Frontiers, 2(1):81-89, 2018.

[283] Michael Kulbak, Satyajit Gupta, Nir Kedem, Igal Levine, Tatyana Bendikov, Gary Hodes, and David Cahen. Cesium enhances long-term 
stability of lead bromide perovskite-based solar cells. The journal of physical chemistry letters, 7(1):167-172, 2015.

[284] Young Soo Kwon, Jongchul Lim, Hui-Jun Yun, Yun-Hi Kim, and Taiho Park. A diketopyrrolopyrrole-containing hole transporting conjugated polymer for use in efficient stable organic-inorganic hybrid solar cells based on a perovskite. Energy \& Environmental Science, 7(4):1454-1460, 2014.

[285] Yingzhuang Ma, Yao-Hsien Chung, Lingling Zheng, Danfei Zhang, Xiao Yu, Lixin Xiao, Zhijian Chen, Shufeng Wang, Bo Qu, Qihuang Gong, et al. Improved hole-transporting property via HAT-CN for perovskite solar cells without lithium salts. ACS applied materials \& interfaces, 7(12):6406-6411, 2015.

[286] Xu Dong, Xiang Fang, Minghang Lv, Bencai Lin, Shuai Zhang, Jianning Ding, and Ningyi Yuan. Improvement of the humidity stability of organic-inorganic perovskite solar cells using ultrathin $\mathrm{Al}_{2} \mathrm{O}_{3}$ layers prepared by atomic layer deposition. Journal of Materials Chemistry A, 3(10):5360-5367, 2015.

[287] Chong Chen, Yong Zhai, Fumin Li, Furui Tan, Gentian Yue, Weifeng Zhang, and Mingtai Wang. High efficiency $\mathrm{CH}_{3} \mathrm{NH}_{3} \mathrm{PbI}_{3}$ : CdS perovskite solar cells with CuInS2 as the hole transporting layer. Journal of Power Sources, 341:396-403, 2017.

[288] R Scheer, R Klenk, J Klaer, and I Luck. CuInS 2 based thin film photovoltaics. Solar Energy, 77(6):777-784, 2004.

[289] R Klenk, J Klaer, R Scheer, M Ch Lux-Steiner, I Luck, N Meyer, and U Rühle. Solar cells based on $\mathrm{CuInS}_{2}$-an overview. Thin Solid Films, 480:509-514, 2005.

[290] Liang Li, T Jean Daou, Isabelle Texier, Tran Thi Kim Chi, Nguyen Quang Liem, and Peter Reiss. Highly luminescent $\mathrm{CuInS}_{2} / \mathrm{ZnS}$ core/shell 
nanocrystals: cadmium-free quantum dots for in vivo imaging. Chemistry of Materials, 21(12):2422-2429, 2009.

[291] Xuejun Gao, Wei Li, Xiaoliang Yang, Xiangliang Jin, and Siguo Xiao. NearInfrared emission of $\mathrm{Er}^{3+}$ sensitized by $\mathrm{Mn}^{4+}$ in $\mathrm{Ca}_{14} \mathrm{Zn}_{6} \mathrm{Al}_{10} \mathrm{O}_{35}$ matrix. The Journal of Physical Chemistry C, 119(50):28090-28098, 2015. 